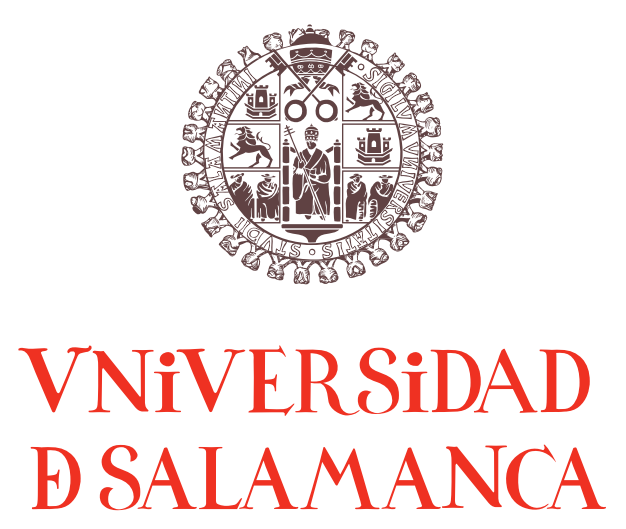

\title{
Teoría y Simulación de Histéresis Angular en Gotas Sésiles
}

\author{
Da María Jesús Santos Sánchez \\ Memoria de Tesis Doctoral
}

Facultad de Ciencias, Salamanca

Dirigida por el

Dr. D. Juan Antonio White Sánchez

Salamanca, 10 de octubre de 2012 



\section{Universidad de Salamanca \\ Departamento de Física Aplicada}

\section{Teoría y Simulación de Histéresis Angular en Gotas Sésiles}

Memoria presentada por Da ${ }^{a}$ María Jesús Santos Sánchez para optar al grado de Doctor por la Universidad de Salamanca. 

D. Juan Antonio White Sánchez, Profesor Titular de Universidad del Departamento de Física Aplicada de la Universidad de Salamanca, autoriza la presentación de la tesis doctoral titulada 'Teoría y simulación de histéresis angular en gotas sésiles', realizada bajo su dirección por $D^{a}$. María Jesús Santos Sánchez.

Salamanca, 10 de octubre de 2012

Fdo.: Juan Antonio White Sánchez 



\section{Agradecimientos}

“Decía Bernardo de Chartres que somos como enanos a los hombros de gigantes. Podemos ver más, y más lejos que ellos, no por alguna distinción física nuestra, sino porque somos levantados por su gran altura."

Juan de Salisbury (1159)

Una Tesis Doctoral supone un trabajo serio, constante y en muchas ocasiones arduo, a lo largo de una serie de años. Que llegue a buen puerto es posible gracias a la dedicación de varias personas que van tejiendo la trama que hace posible esta realidad. En mi caso he tenido la suerte de hallarme "a hombros de gigantes", grandes físicos y mejores personas para los que solo puedo tener palabras de agradecimiento.

Gracias a Juan Antonio White, mi director de tesis, por su dedicación, paciencia, y el apoyo continuo que ha mostrado en todo momento.

Gracias a Santiago Velasco por los matices aportados, y por todas y cada una de sus enseñanzas.

Y gracias a los miembros del Grupo de Termodinámica y Física Estadística de la Universidad de Salamanca, pues está constituido por personas siempre dispuestas a ayudar de forma desinteresada.

María Jesús Santos Sánchez 

A Pablo y Aníbal 



\section{Índice general}

$\begin{array}{ll}\text { Índice de símbolos } & 17\end{array}$

1. Conceptos básicos 19

1.1. Presentación . . . . . . . . . . . . . . . . . . . . . 19

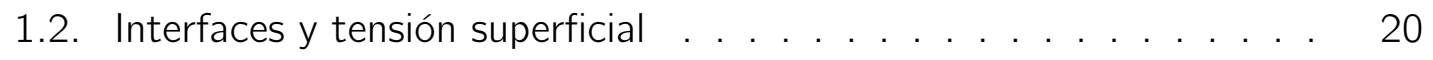

1.2.1. Interfaz . . . . . . . . . . . . . . . 20

1.2.2. Energía asociada a una superficie . . . . . . . . . . 21

1.2.3. Tensión superficial . . . . . . . . . . . . . . . . 22

1.3. Ley de Laplace y sus aplicaciones . . . . . . . . . . . . . . . . 24

1.3.1. Radio de curvatura y curvatura . . . . . . . . . . . . . 24

1.3.2. Deducción de la Ley de Laplace . . . . . . . . . . . . 25

1.3.3. Ecuación de Young-Laplace en gravedad . . . . . . . . . 27

1.4. Mojado de superficies . . . . . . . . . . . . . . . . . . . . . . . 29

1.4.1. Ecuación de Young. Ángulo de contacto de Young . . . . 3 30

1.4.2. Medida del ángulo de contacto de gotas sésiles . . . . . . 32

1.5. Minimización de la Energía Superficial . . . . . . . . . . . 34

1.6. Gotas en superficies no homogéneas . . . . . . . . . . . . . . . . . . . . . . . 35

1.6.1. Ley de Wenzel . . . . . . . . . . . . . . . . . . . . 35

1.6.2. Ley de Cassie . . . . . . . . . . . . . . . . . . . . 36

1.7. Histéresis angular . . . . . . . . . . . . . . . . . . . . 37

1.8. Gotas sésiles con Surface Evolver . . . . . . . . . . . . . . 38

1.8.1. Surface Evolver . . . . . . . . . . . . . . . . . . . . . . 39

1.8.2. Energía en Surface Evolver . . . . . . . . . . . . . . . 41

2. Histéresis en gotas axisimétricas 45

2.1. Introducción . . . . . . . . . . . . . . . . . . 45

2.2. Solución de la ecuación de Young-Laplace para gotas axisimétricas . 47 
2.2.1. Simulación de un ciclo de histéresis . . . . . . . . . . . . 52

2.3. Implementación en Surface Evolver . . . . . . . . . . . . . . . . 56

2.3.1. Fuerza de fricción . . . . . . . . . . . . . . . . . . . 57

2.3.2. Medida del ángulo de contacto . . . . . . . . . . . . . 58

2.4. Comparación con resultados experimentales . . . . . . . . . . . 63

2.4.1. Comparación con los resultados de Lam et al. . . . . . . . . 63

2.4.2. Comparación con los resultados de Vargha-Butler el al. . . . 69

2.5. Conclusiones . . . . . . . . . . . . . . . . . . . . . . . 71

3. Histéresis en gotas sobre superficies inclinadas 73

3.1. Introducción . . . . . . . . . . . . . . . . . . . . 73

3.2. Método de simulación . . . . . . . . . . . . . . . . . . 78

3.3. Simulación de un proceso de inclinación . . . . . . . . . . . . . 82

3.3.1. Ángulo de contacto inicial igual a $\theta_{\mathrm{av}}$. . . . . . . . . . . 83

3.3.2. Ángulo de contacto inicial igual a $\theta_{Y}$. . . . . . . . . . . . . 96

3.4. Comparación con datos experimentales . . . . . . . . . . . . . 100

3.5. Análisis de la deformación de gotas sobre superficies inclinadas . . . 103

3.5.1. Relación de aspecto $\beta$ frente a la diferencia de $\operatorname{cosenos} \cos \theta_{\min }-$ $\cos \theta_{\max } \ldots \ldots . . \ldots 103$

3.5.2. Relación entre el radio medio $R_{\mathrm{a}}$, el semiancho $\omega$ y la relación

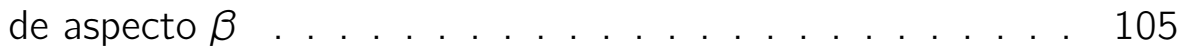

3.5.3. Relación entre la fuerza y el área de la base de la gota . . . 109

3.6. Conclusiones. . . . . . . . . . . . . . . . . . . . . . . . . . . . 112

4. Ángulo de contacto más estable $\quad 117$

4.1. Introducción . . . . . . . . . . . . . . . . . . . 117

4.2. Método de placa inclinable . . . . . . . . . . . . . . . . . . 118

4.2.1. Resultados ... . . . . . . . . . . . . . . 120

4.3. Método de vibraciones mecánicas . . . . . . . . . . . . . . . . . 130

4.3.1. Resultados . . . . . . . . . . . . . . . . . . 137

4.4. Comparación de resultados . . . . . . . . . . . . . . . . . . . . 137

4.5. Conclusiones. . . . . . . . . . . . . . . . . . . . . . . . . 143 
5. Conclusiones 145

A. Ecuación de Young-Laplace para gotas axisimétricas $\quad \mathbf{1 4 9}$

A.1. Ecuación de Young-Laplace en coordenadas cilíndricas . . . . . . . 149

$\begin{array}{ll}\text { Índice de figuras } & 163\end{array}$

$\begin{array}{ll}\text { Índice de cuadros } & 173\end{array}$ 



\section{Nomenclatura}

$\alpha \quad$ ángulo de inclinación de la superficie respecto a la horizontal

$\alpha_{1} \quad$ ángulo de inclinación para el que se observa el primer desplazamiento de la línea de contacto (ya sea la frontal o la posterior) durante el proceso de inclinación

$\alpha_{2} \quad$ ángulo mínimo de inclinación para el que tanto la parte posterior como la delantera de la línea de contacto se han desplazado

$\alpha_{c} \quad$ ángulo de inclinación crítico

$\alpha_{\mathrm{d}} \quad$ ángulo de inclinación crítico para el que se observa el desplazamiento de la parte delantera

$\alpha_{\mathrm{u}} \quad$ ángulo de inclinación crítico para el que se observa el desplazamiento de la parte posterior

$\bar{\theta} \quad$ ángulo de contacto medio

$\beta \quad$ relación de aspecto de la gota

$\hat{k} \quad$ curvatura

$\hat{r} \quad$ rugosidad

$\rho \quad$ densidad de un fluido

$\sigma \quad$ densidad de energía superficial

$\sigma_{\mathrm{Is}} \quad$ tensión superficial de la interfaz líquido-sólido

$\sigma_{\mathrm{lv}} \quad$ tensión superficial de la interfaz líquido-vapor

$\sigma_{\mathrm{sv}} \quad$ tensión superficial de la interfaz sólido-vapor

$\theta^{*} \quad$ ángulo de contacto con una superficie rugosa 
$\theta \quad$ ángulo de contacto

$\theta_{\mathrm{av}}, \theta_{\mathrm{re}}$ ángulos de contacto de avance y de retroceso

$\theta_{\text {front }}$ ángulo de contacto de la parte frontal de la gota

$\theta_{\text {ini }}, \theta_{0}$ ángulo de contacto inicial

$\theta_{\text {me }} \quad$ ángulo de contacto más estable

$\theta_{\min }, \theta_{\max }$ ángulos de contacto mínimo y máximo de la gota inclinada

$\theta_{\text {post }}$ ángulo de contacto de la parte posterior de la gota

$\theta_{Y} \quad$ ángulo de Young o ángulo de contacto intrínseco

A área de la superficie de la interfaz

$A_{1}, A_{2}$ fracciones de superficie de dos materiales

$A_{\mathrm{ls}} \quad$ área del líquido en contacto con la superficie (el área de la base de la gota)

$A_{l v} \quad$ área de la interfaz líquido-vapor

$A_{\mathrm{sv}, 0}$ área de la superficie del substrato

c constante de capilaridad

f $\quad$ fuerza de fricción por unidad de longitud

$F_{y} \quad$ fuerza de retención total a lo largo de la dirección de inclinación

$g \quad$ aceleración debida a la gravedad

k factor de retención

I0 longitud de capilaridad

$I_{i, 1}, I_{i, 2}$ longitudes de los dos ejes adyacentes al vértice $i$ que pertenecen a la línea de contacto triple 
p presión

$R, \varphi, z$ coordenadas cilíndricas

$r_{0} \quad$ radio de curvatura en el ápice

$R_{1}, R_{2}$ radios (principales) de curvatura

$R_{\mathrm{a}} \quad$ radio equivalente de un círculo con el mismo área que el de la base de la gota

S parámetro de expansión (spreading)

$s \quad$ longitud de arco

U energía de una superficie

$U_{\text {lv }} \quad$ energía de la superficie líquido-vapor

$U_{\text {sl }} \quad$ energía de la base líquido-sólido

$U_{\mathrm{sv}, 1}$ energía superficial de la superficie del sólido en contacto con el vapor

$\checkmark \quad$ volumen

$V_{\mathrm{g}} \quad$ volumen de la gota

$V_{\text {ini }}, V_{\text {fin }}$ volumen inicial y volumen final

W trabajo

$\mathcal{H}$ curvatura media

$\mathcal{H}_{\Sigma} \quad$ curvatura media de la superficie descrita por la parametrización de la interfaz $\Sigma$ en un punto cualquiera de ésta 



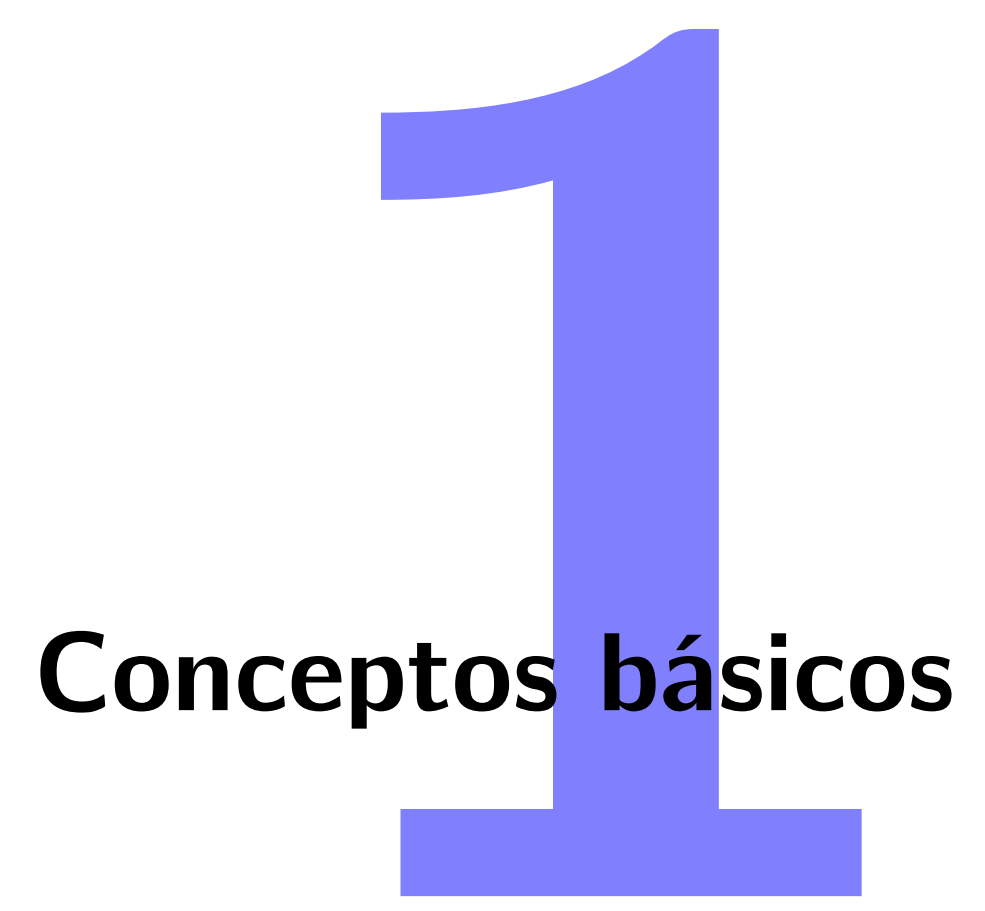

\subsection{Presentación}

A comienzos del siglo XIX Thomas Young (1805) y Pierre-Simon Laplace (1806) establecieron las bases de la teoría clásica de la capilaridad que explica fenómenos como la aparición de meniscos o la forma de las gotas de agua. A pesar de haber transcurrido más de 200 años desde la publicación de los trabajos de Young [1] y Laplace [2], los fenómenos asociados con la capilaridad siguen siendo de notable interés en la actualidad debido a que dan lugar a una gran cantidad de aplicaciones prácticas en campos como la Ciencia de Materiales y la Biología. Así mismo, estos fenómenos son fundamentales en dispositivos microfluídicos donde los efectos de superficie juegan un papel dominante [3].

El reciente interés en las superficies superhidrófobas [4] en las que las gotas resbalan sin la necesidad de grandes ángulos de inclinación, ha puesto el énfasis en la necesidad de entender los límites de estabilidad de este tipo de sistemas. Las aplicaciones prácticas de estos estudios van desde el diseño de parabrisas, cubiertas de invernaderos o paneles solares, hasta la necesidad de que los insecticidas líquidos queden retenidos en las hojas de los cultivos $[4,5]$.

Una de las principales dificultades a la hora de tratar problemas referentes a 
superficies de contacto e interfaces en general, proviene de su tratamiento numérico. El cálculo y minimización del funcional de energía del problema lleva a la necesidad de resolver las ecuaciones de Euler-Lagrange con las condiciones de contorno adecuadas. Los métodos existentes para la minimización del funcional de energía están basados principalmente en el uso de técnicas de elementos finitos y su implementación requiere en ocasiones de software comercial. En particular, muchos trabajos de los últimos años emplean el software de dominio público Surface Evolver [6].

En este trabajo se propone un modelo sencillo para simular histéresis de ángulos de contacto en gotas líquidas sobre superficies planas e inclinadas. Las simulaciones se han realizado mediante un método basado en el uso de Surface Evolver. El modelo supone la existencia de una fuerza de fricción actuando sobre la línea de contacto triple de modo que ésta permanece fija para ángulos comprendidos entre el ángulo de avance y el de retroceso, y permite que se mueva en caso contrario.

Este capítulo está dedicado a la presentación de conceptos básicos para el posterior desarrollo de nuestro estudio. Se comentarán brevemente conceptos como interfaz, energía superficial, tensión superficial, línea de contacto triple, ley de Laplace, ángulo de contacto y ecuación de Young, entre otros, que permitirán entender la forma de una gota depositada sobre la superficie de un sólido (gota sésil) y los fenómenos de histéresis angular que se observan en aplicaciones prácticas. Se presenta además la herramienta empleada en las simulaciones: el software libre Surface Evolver [6].

\subsection{Interfaces y tensión superficial}

\subsubsection{Interfaz}

Cuando se desea insistir en la naturaleza de una superficie, como frontera entre dos sustancias, se habla de interfaz. Matemáticamente una interfaz es la superficie geométrica que delimita dos fluidos. Esta definición implica que una interfaz no tiene espesor y que es suave (es decir, no es rugosa). En la práctica esta definición es un concepto esquemático. La realidad es más compleja y la separación de dos fluidos 
inmiscibles (agua-aire, agua-aceite, etc.) depende de las interacciones moleculares de cada fluido [7] y de la difusión Browniana (agitación térmica). Por lo tanto las características de las interfaces se deben en gran medida a las de los dos medios que la forman [3].

En un sistema heterogéneo, se denomina fase a cada una de las partes homogéneas que lo constituyen. La interfase se podría definir como la región intermedia entre dos fases, de manera que tiene la misma ubicación que la interfaz. Ésta última, la interfaz, sin embargo, pone de manifiesto la naturaleza bidimensional de la región en su descripción y es ésta la que se usa comúnmente entre los científicos que estudian fenómenos superficiales, mientras que interfase se utiliza habitualmente en Biología en la descripción de los ciclos celulares [8].

\subsubsection{Energía asociada a una superficie}

La existencia de una superficie no es algo favorable desde el punto de vista energético. Su extensión se ve reducida en la medida de lo posible debido a que la creación de una superficie supone un coste energético. La energía asociada a la superficie se puede expresar como el producto del área de dicha superficie multiplicada por una densidad de energía superficial o densidad de energía interfacial, según se trate de superficies o interfaces.

La densidad de energía superficial suele simbolizarse con la letra griega $\sigma$, y tiene unidades de energía por unidad de área, $\mathrm{J} / \mathrm{m}^{2}$ en el Sistema Internacional. Para indicar que se trata de la densidad de energía de una interfaz entre dos medios A y B se simboliza como $\sigma_{\mathrm{AB}}$.

El coste energético asociado a la formación de interfaces tiene una gran influencia en la naturaleza, creando una dependencia muy significativa de las propiedades de los sistemas con el tamaño, debido a la distinta influencia entre los fenómenos relativos a las áreas y a los volúmenes implicados en el sistema. 


\section{Interpretación microscópica de la energía superficial}

La energía superficial es consecuencia de las fuerzas de cohesión internas. Para comprender su naturaleza se debe descender a la descripción microscópica de las interfaces.

Las moléculas localizadas en el seno de un líquido tienen interacciones con todas las moléculas vecinas; estas interacciones son en su mayoría fuerzas atractivas de Van der Waals para los líquidos orgánicos y cadenas de hidrógeno para los líquidos polares como el agua [7]. Sin embargo las moléculas de una interfaz tienen interacciones por un lado con moléculas del mismo líquido y por otro interacciones con moléculas de otro fluido.

Si se considera una interfaz entre un líquido y un gas, debido a la baja densidad de los gases, hay menos interacciones en el lado de éstos que en el lado del líquido. El resultado es que existe una asimetría local en las interacciones de manera que las moléculas de la interfaz siempre buscan otras moléculas iguales para equilibrar sus interacciones, y de esta manera reducir la energía superficial. Una consecuencia de esto es que se tiende a minimizar el área de la interfaz. Dicho de otra manera, es energéticamente costoso crear o aumentar una interfaz. El mismo razonamiento se puede aplicar a dos líquidos, excepto que las interacciones con el otro líquido suelen ser más energéticas que con el gas y la asimetría resultante será menor.

Íntimamente ligado a la energía superficial se encuentra el concepto de tensión superficial que se pasa a describir a continuación.

\subsubsection{Tensión superficial}

Debido al coste energético de las superficies (la energía superficial) aparece una resistencia de éstas a aumentar su tamaño. Esta resistencia se traduce en la aparición de una fuerza por unidad de longitud que es tangencial a la superficie y tiende a reducir su extension. La nueva magnitud se denomina tensión superficial y se simboliza habitualmente con la letra griega $\gamma$. La tensión superficial tiene unidades de fuerza por unidad de longitud, N/m en el Sistema Internacional, dimensionalmente análogas a las de la densidad de energía superficial. 


\begin{tabular}{cc}
\hline Líquido & Tensión superficial $\mathbf{( m N} / \mathbf{m})$ \\
\hline Agua & 72,8 \\
Acetona & 25,2 \\
Benceno & 28,9 \\
Cloroformo & 27,1 \\
Etanol & 22,1 \\
Etilenglicol & 47,7 \\
Formamida & 59,1 \\
Glicerol & 64,0 \\
Hexano & 18,5 \\
Hexadecano & 27,6 \\
Mercurio & 425,4 \\
\hline
\end{tabular}

Cuadro 1.1: Valores típicos de la tensión superficial de algunos líquidos puros a temperatura de $20^{\circ} \mathrm{C}[3,8]$.

Esta equivalencia entre la tensión superficial y la densidad de energía superficial no es sólo dimensional, sino que el valor de una coincide con el de la otra, aunque conceptualmente sean interpretadas de manera distinta. El trabajo necesario para aumentar la interfaz una unidad de área coincide con el aumento de energía de la interfaz.

Habitualmente se hace referencia a la densidad de energía superficial característica de las interfaces con el nombre de tensión superficial, tanto para insistir en su naturaleza energética como mecánica. En este texto se hace uso tanto de un término como de otro y se simboliza la tensión superficial con la letra griega $\sigma$ en lugar de $\gamma$.

Es conveniente indicar que el término tensión superficial se utiliza a menudo para referirse a la tensión superficial de una sustancia en equilibrio con su vapor, mientras que se deja el término tensión interfacial para aquella entre dos sustancias distintas [3]. Por ejemplo, se dice tensión superficial del agua y tensión interfacial aguaaceite. En el cuadro 1.1 se pueden observar valores típicos de la tensión superficial para algunos líquidos a temperatura de $20^{\circ} \mathrm{C}$.

Puede observarse que la tensión superficial aumenta a medida que la atracción 
molecular aumenta y el tamaño molecular disminuye. Para la mayoría de los aceites, el valor de la tensión superficial se encuentra en el intervalo de $20-30 \mathrm{mN} / \mathrm{m}$, mientras que para el agua es alrededor de $70 \mathrm{mN} / \mathrm{m}$. Los valores más altos de la tensión superficial son para los metales líquidos, por ejemplo, el mercurio líquido tiene una tensión superficial del orden de $500 \mathrm{mN} / \mathrm{m}$.

De acuerdo con la definición de tensión superficial, la energía total $U_{\mathrm{T}}$ de una superficie homogénea es

$$
U_{\mathrm{T}}=\sigma A
$$

donde $A$ es el área de la superficie de la interfaz y $\sigma$ la tensión superficial.

Aquellos fluidos que tienen tensión interfacial cero $(\sigma=0)$ se dice que son miscibles.

\subsection{Ley de Laplace y sus aplicaciones}

La ley de Laplace, también Ilamada ecuación de Young-Laplace, relaciona el cambio de presiones en la superficie que separa dos fluidos con la curvatura de dicha superficie. Antes de presentar una demostración sencilla de esta ley se comenzará introduciendo la noción de curvatura.

\subsubsection{Radio de curvatura y curvatura}

El radio de curvatura en una curva plana es el radio $R$ del círculo osculante, es decir el círculo que mejor describe la curva en el punto de contacto (figura 1.1). Dada una curva plana, se define su curvatura, $\hat{k}$ como:

$$
\hat{k}=\frac{1}{R}
$$

tanto la curvatura $\hat{k}$ como el radio de curvatura $R$ son cantidades con signo. El radio de curvatura puede ser positivo o negativo, dependiendo de la orientación (convexa o cóncava) de la curva:

$$
\left\{\begin{array}{l}
R>0 \Rightarrow \hat{k}>0 \Leftrightarrow \text { convexa } \\
R<0 \Rightarrow \hat{k}<0 \Leftrightarrow \text { cóncava }
\end{array}\right.
$$




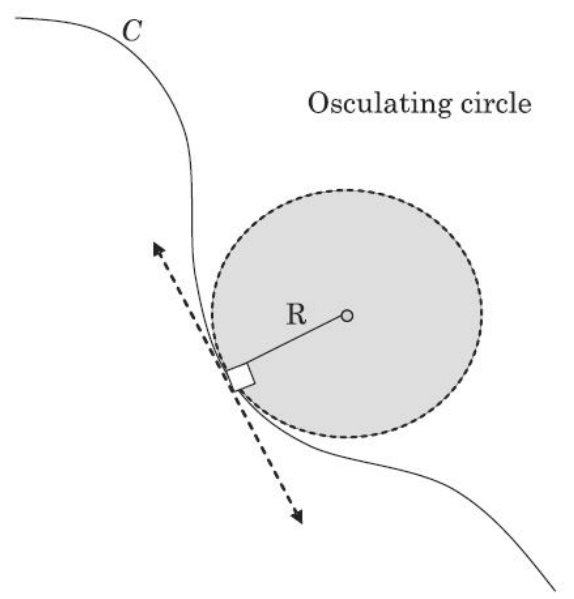

Figura 1.1: Radio de curvatura y círculo osculante. Imagen tomada de [3]

La curvatura media $\mathcal{H}$ de una superficie se define utilizando sus curvaturas principales (máxima y mínima) $\hat{k}_{1}$ y $\hat{k}_{2}$ dentro de todo el conjunto de curvaturas:

$$
\mathcal{H}=\frac{1}{2}\left(\hat{k}_{1}+\hat{k}_{2}\right)=\frac{1}{2}\left(\frac{1}{R_{1}}+\frac{1}{R_{2}}\right) .
$$

Una vez introducido el concepto de curvatura, se pasa a deducir de un modo sencillo la Ley de Laplace.

\subsubsection{Deducción de la Ley de Laplace}

Considérese una gota esférica de un líquido rodeada de un fluido. A continuación se va a calcular el trabajo $W$ necesario para aumentar su volumen $V$ desde un radio $R$ a un radio $R+d R$ (figura 1.2). La parte del trabajo debida a que el volumen interno aumenta es:

$$
\delta W_{i}=-p_{0} d V_{0}
$$

donde $d V_{0}=4 \pi R^{2} d R$ es el volumen que ha aumentado la gota y $p_{0}$ es la presión inicial de dicha gota.

El trabajo para desplazar el fluido externo es:

$$
\delta W_{\mathrm{e}}=-p_{1} d V_{1}
$$




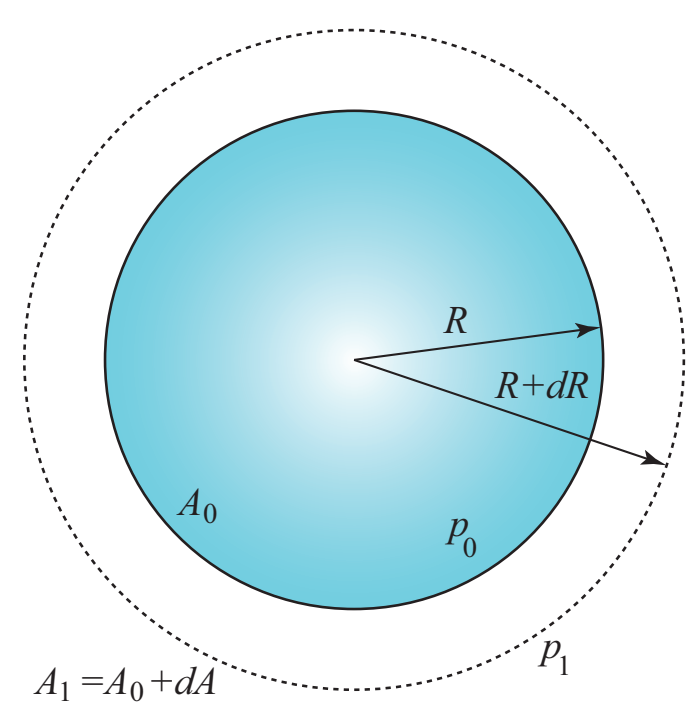

Figura 1.2: Esquema de una gota de líquido inmersa en un fluido; inicialmente, el radio de la gota es $R$ y su área superficial es $A_{0}$. Un aumento de su radio en una cantidad $d R$ se corresponde con un nuevo área superficial $A_{1}$ y una presión $p_{1}$.

donde $d V_{1}$ es la disminución del volumen externo que es igual a $-d V_{0}$, por lo tanto: $\delta W_{\mathrm{e}}=+p_{1} d V_{0}$.

El trabajo correspondiente al aumento de la superficie $A$ de la interfaz es:

$$
\delta W_{\mathrm{s}}=\sigma d A,
$$

donde $d A=8 \pi R d R$ es la variación del área de la superficie esférica.

La condición de equilibrio mecánico se puede escribir como:

$$
\delta W=\delta W_{\mathrm{i}}+\delta W_{\mathrm{e}}+\delta W_{\mathrm{s}}=0
$$

Sustituyendo en la ecuación (1.7) los valores de los trabajos obtenidos previamente, se deduce

$$
\Delta p=p_{0}-p_{1}=\frac{2 \sigma}{R} .
$$

La expresión (1.8) es la ecuación de Laplace para una esfera.

El razonamiento utilizado para obtener la ecuación (1.8) se puede generalizar para una interfaz localmente definida por dos radios (principales) de curvatura $R_{1}$ y 
$R_{2}$, obteniendo:

$$
\Delta p=\sigma\left(\frac{1}{R_{1}}+\frac{1}{R_{2}}\right) .
$$

La expresión (1.9) es conocida como la ecuación de Laplace [2]. Está íntimamente ligada a la minimización de la energía superficial y es la ecuación fundamental a la hora de trabajar con interfaces, microgotas así como en microfluídica digital.

Teniendo en cuenta la definición de la curvatura media (1.3), se puede reescribir la ecuación de Laplace de la siguiente manera:

$$
\mathcal{H}=\frac{1}{2} \frac{\Delta p}{\sigma},
$$

donde $\mathcal{H}$ representa la curvatura media de un punto cualquiera de la interfaz, $\Delta p$ es la diferencia de presión de los fluidos a ambos lados de la interfaz, y $\sigma$ la densidad de la energía superficial. Esta expresión se conoce como ecuación de Young-Laplace o ecuación de Laplace de la capilaridad y expresa formalmente que la curvatura media en cualquier punto de la interfaz es proporcional a la diferencia de presión entre los fluidos e inversamente proporcional a su densidad de energía interfacial.

Se considerará a continuación la ecuación de Young-Laplace para un sistema en un campo gravitatorio.

\subsubsection{Ecuación de Young-Laplace en gravedad}

Si se considera un sistema formado por dos fluidos separados por una interfaz, la diferencia de presión a los lados de la interfaz variará con la altura de acuerdo con la ecuación de la hidrostática:

$$
\Delta p(h)=\Delta p_{0}-\Delta \rho g h,
$$

donde $\Delta p_{0}$ es la diferencia de presión a los lados de la interfaz para el punto de altura $h$ cero, $\Delta \rho$ es la diferencia de densidad entre los fluidos y $g$ la aceleración debida a la gravedad.

De acuerdo con las expresiones (1.10) y (1.11) se puede escribir la ecuación de Young-Laplace para un sistema sometido a la acción de la gravedad de la forma:

$$
\mathcal{H}_{\Sigma}(u, v)=\frac{\Delta p_{0}}{2 \sigma}-\frac{\Delta \rho g h(u, v)}{2 \sigma},
$$


donde $\mathcal{H}_{\Sigma}$ representa la curvatura media de la superficie descrita por la parametrización de la misma $\Sigma$ en un punto cualquiera $(u, v)$. Esta expresión es una ecuación diferencial de segundo orden en derivadas parciales donde $\mathcal{H}_{\Sigma}(u, v)$ contiene las derivadas de la ecuación.

El término $\Delta p_{0} / 2 \sigma$ en la ecuación (1.12) corresponde a la curvatura media de la interfaz en un punto de altura cero, de manera que se puede definir el radio medio de curvatura [ecuación (1.8)] en el punto de altura cero, $r_{0}$, como:

$$
\frac{1}{r_{0}} \equiv \frac{\Delta p_{0}}{2 \sigma}
$$

En las superficies de revolución, como es el caso de algunas gotas, es común tomar como origen de alturas el ápice de la gota, de manera que este radio medio de curvatura se convierte en el radio de curvatura (único dada la simetría de revolución de la gota) en el ápice.

El término gravitatorio en la ecuación (1.12) puede ser reescrito de modo sencillo usando el concepto de longitud de capilaridad

$$
\iota_{0} \equiv \sqrt{\frac{\sigma}{\Delta \rho g}} .
$$

Este parámetro es una longitud característica que determina el balance entre términos de superficie y gravitatorios en un fluido ${ }^{1}$. Una manera sencilla de analizar este concepto es a partir del cociente entre la variación de presión debida a la tensión superficial y la variación de presión con la altura. Sea una sistema con unas dimensiones lineales del orden de $I$. En este caso, la variación de presión debida a la tensión superficial es aproximadamente igual a $\sigma / /$ mientras que la variación con la altura es aproximadamente igual a $\Delta \rho g l$. Por tanto

$$
\frac{\text { variación de presión debida a la tensión superficial }}{\text { variación de presión debida a la altura }} \approx \frac{\sigma / l}{\Delta \rho g l}=\frac{l_{0}^{2}}{I^{2}} \text {. }
$$

Con lo que en un sistema con dimensiones mucho menores que la longitud de capilaridad dominan efectos debidos a la tensión superficial y, al contrario, en un sistema

\footnotetext{
${ }^{1}$ La longitud de capilaridad es del orden de $2 \mathrm{~mm}$ para la mayoría de los líquidos, incluso para el mercurio.
} 


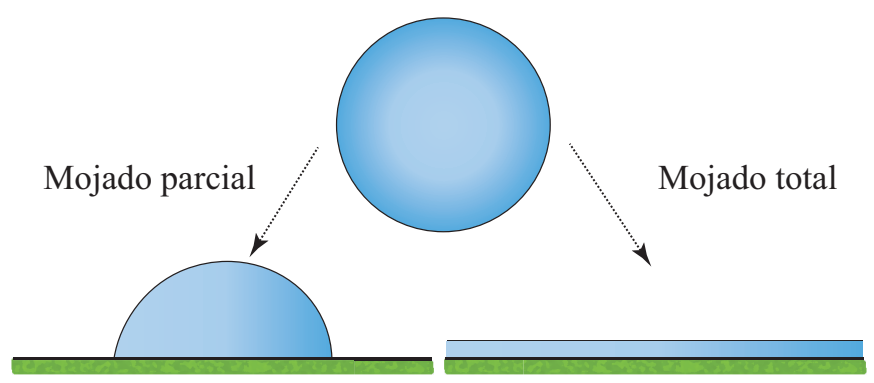

Figura 1.3: Cuando el líquido se extiende como una película sobre la superficie sólida se habla de mojado total.

con dimensiones mucho mayores que la longitud de capilaridad dominan efectos de tipo gravitatorio.

A partir de las definiciones (1.13) y (1.14) la ecuación de Young-Laplace (1.12) puede escribirse del siguiente modo:

$$
\mathcal{H}_{\Sigma}(u, v)=\frac{1}{r_{0}}-\frac{h(u, v)}{2 I_{0}{ }^{2}} .
$$

\subsection{Mojado de superficies}

Los líquidos sobre una superficie horizontal se comportan de forma diferente según la naturaleza tanto de la superficie sólida como del líquido. En realidad, esto depende de los tres constituyentes, según sea el gas o el fluido que rodea a la gota. Son posibles dos situaciones diferentes: si el líquido forma una gota se dice que el mojado es parcial, si el líquido forma una película delgada mojando la superficie sólida se habla de mojado total, con la dimensión horizontal de la película dependiendo del volumen inicial del líquido (figura 1.3). Por ejemplo, en el ambiente de una habitación, el agua se extiende como una película sobre una superficie muy limpia y suave de un cristal, mientras que forma una gota sobre un plástico. En el caso del mojado parcial, existe una línea en la cual las tres fases se unen, ésta se denomina línea de contacto triple o línea triple; por ejemplo, una gota de agua sobre un substrato sólido tiene una línea de contacto triple teniendo en cuenta el gas que rodea a ambos. 


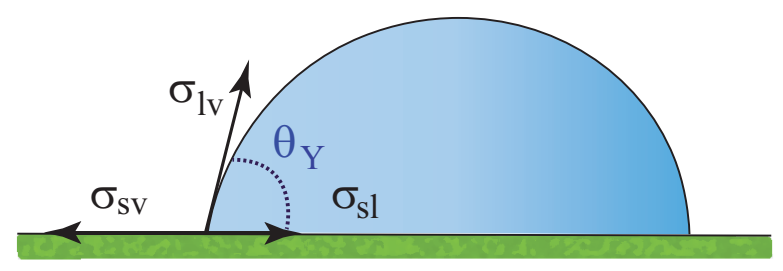

Figura 1.4: Esquema de la región de contacto de una gota depositada sobre la superficie de un sólido. Las tensiones interfaciales son representadas vectorialmente tangentes a su interfaz. A partir de la componente horizontal del equilibrio de fuerzas se deduce la ecuación de Young.

Un líquido se extiende sobre un substrato en forma de película si la energía del sistema es menor debido a la presencia de la película de líquido. La energía superficial por unidad de superficie del sólido seco es $\sigma_{\text {sv }}$ y la energía superficial del sólido mojado es $\sigma_{\mathrm{sl}}+\sigma_{\mathrm{lv}}$. El parámetro de expansión (spreading) $S$ determina el tipo de expansión (total o parcial):

$$
S=\sigma_{\mathrm{sv}}-\left(\sigma_{\mathrm{sl}}+\sigma_{\mathrm{lv}}\right)
$$

de modo que:

$\left\{\begin{array}{l}\text { Si } S>0 \Rightarrow \text { el líquido se extiende sobre la superficie del sólido, } \\ \text { Si } S<0 \Rightarrow \text { el líquido forma una gota sobre la superficie del sólido. }\end{array}\right.$

Este trabajo se centra en situaciones de mojado parcial, es decir, cuando el líquido forma una gota sobre el substrato en el que se encuentra. El siguiente subapartado aborda la ecuación de Young, que explica dicho comportamiento y los elementos que la definen, entre ellos el ángulo de contacto.

\subsubsection{Ecuación de Young. Ángulo de contacto de Young}

La tensión superficial representa una fuerza por unidad de longitud que se ejerce tangencialmente a la interfaz. En la figura 1.4 se muestra un esquema de las fuerzas 
por unidad de longitud que se ejercen en la línea de contacto triple de una gota depositada sobre un substrato. En el equilibrio la resultante de las fuerzas debe ser nula. Utilizando un sistema de coordenadas donde el eje $x$ es tangente a la superficie del sólido en la línea de contacto (horizontal) y el eje y es perpendicular (vertical), en el equilibrio, la proyección de la resultante sobre el eje $x$ es cero y se obtiene la Ecuación de Young, deducida por Thomas Young en 1805 [1]:

$$
\sigma_{\mathrm{sv}}=\sigma_{\mathrm{sl}}+\sigma_{\mathrm{lv}} \cos \theta_{\mathrm{Y}}
$$

donde $\theta_{Y}$ es al ángulo de contacto de Young, es decir, el ángulo que forman las interfaces sólido-líquido y líquido-vapor en un punto cualquiera de la línea de contacto triple. A la hora de establecer esta ecuación se supone que la superficie del sólido es suave, homogénea y rígida; además debe ser química y físicamente inerte respecto a los líquidos empleados. Es decir, se trata de una superficie ideal.

El ángulo de contacto de Young, en ocasiones también llamado ángulo de contacto intrínseco, es el ángulo de contacto que exhibiría el líquido al mojar un punto de la superficie ideal, debido únicamente a las energías superficiales implicadas en el sistema, es decir, de acuerdo con la ecuación de Young. En condiciones no-ideales el ángulo de contacto real $\theta$ puede diferir notablemente del ángulo de contacto de Young $\theta_{Y}$.

Cuando el líquido no moja totalmente al sólido, se forma una gota en su superficie. Se pueden presentar dos situaciones (figura 1.5): si el ángulo de contacto con el sólido es menor de $90^{\circ}$ se habla de superficie hidrófila si el líquido es agua o, en general, superficie que moja ("wetting"). En el caso opuesto, de un ángulo de contacto con el sólido mayor de $90^{\circ}$ se dice que la superficie es hidrófoba si se trata de agua o en general que no moja ("not wetting").

$$
\left\{\begin{array}{l}
\text { Si } \theta_{Y}<90^{\circ} \Rightarrow \text { superficie hidrófila } \\
\text { Si } \theta_{Y}>90^{\circ} \Rightarrow \text { superficie hidrófoba }
\end{array}\right.
$$

En experiencias reales, cuando se trabaja con líquidos biológicos, se pueden observar cambios con el tiempo en el ángulo de contacto. Esto es debido a que los líquidos biológicos no son homogéneos y pueden depositar una capa de moléculas 
a) Líquido moja al sólido $\left(\theta_{\mathrm{Y}}<90^{\circ}\right)$

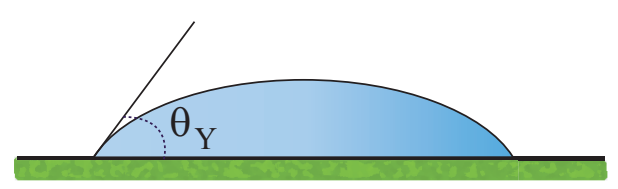

b) Líquido no moja al sólido

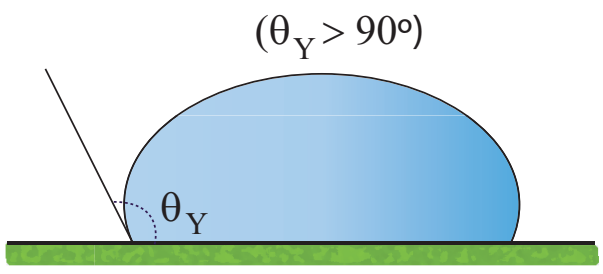

Figura 1.5: a) Superficie hidrófila con $\theta_{Y}<90^{\circ}$ y b) superficie hidrófoba con $\theta_{Y}>90^{\circ}$.

sobre la pared sólida, modificando progresivamente el valor de la tensión superficial $\sigma_{\mathrm{sl}}$, y consecuentemente el valor de $\theta_{\mathrm{Y}}$, tal y como aparece en la ecuación de Young.

El ángulo de contacto juega un papel fundamental para la caracterización de la forma de una gota y, por lo tanto, en el comportamiento de un líquido en contacto con un substrato determinado. A continuación se explica brevemente cómo se aborda experimentalmente la medida de dicho ángulo.

\subsubsection{Medida del ángulo de contacto de gotas sésiles}

Tradicionalmente, la medida del ángulo de contacto se ha efectuado por medio de un dispositivo experimental consistente en un goniómetro acompañado de un microscopio. Este dispositivo permite medir el ángulo de contacto de las gotas a partir de una vista lateral de las mismas.

El análisis de la forma de gotas con simetría axial (axisimétricas) para determinar la tensión superficial de un líquido fue popularizado en la década de los 90 del siglo pasado por los científicos canadienses A. Wilhelm Neumann, Daniel Y. Kwok y Oscar I. del Rio entre otros $[9,10]$. Sus siglas en inglés ADSA (Axisymmetric Drop Shape Analysis) se utilizan hoy a menudo para designar tanto esta técnica como su metodología, extendiéndose el uso de esta técnica hasta el punto de que, a menudo, se considera técnica ADSA toda técnica que examine la forma de una gota, no sólo para la determinación de la tensión superficial, sino también en la medida de ángulos 
de contacto, volúmenes, etc.

Partiendo de esta metodología, es frecuente el uso de otras dos, ADSA-P y ADSA-D, que son derivadas de ella y que pasamos a describir brevemente.

\section{ADSA-P, análisis del perfil de gotas sésiles}

A menudo se recurre a una medida indirecta de los ángulos de contacto a partir de la forma completa de la gota, para obtener mayor exactitud. Se puede ajustar el perfil de una gota sésil obteniendo una curva cuya pendiente en el punto de contacto ha de coincidir con la tangente del ángulo de contacto. Esta forma de medir sustituye a la medida directa, pues en realidad, ni siquiera sería necesario poder observar la región de contacto con nitidez, basta tener suficientes puntos para encontrar la curva que mejor se ajusta a la gota y conocer la altura de la misma, es decir la situación del ápice de la gota y la altura de la línea horizontal que representa la superficie. Por su similitud con la metodología desarrollada para la determinación de la tensión superficial se denota a menudo con las siglas ADSA-P (Axisymmetric Drop Shape Analysis Profile) [11].

\section{ADSA-D, análisis de la superficie de contacto}

En ocasiones las gotas mojan las superficies de los sólidos sobre las que han sido depositadas de tal manera que subtienden un ángulo de contacto muy pequeño (incluso del orden de unos pocos grados), lo que supone una dificultad en el análisis de los perfiles y en la precisión en la determinación del ángulo de contacto.

En este caso, en lugar de medir directamente el ángulo de contacto, se recurre a un análisis de la gota con una vista desde arriba con la intención de determinar con exactitud dicho radio de contacto y a partir de él y del valor conocido del volumen de la gota y de la tensión superficial del líquido, se hace una estimación del ángulo de contacto de la gota.

La precisión de esta técnica disminuye cuando las gotas sésiles abandonan su simetría axial o cuando la tensión superficial o el volumen de la gota son susceptibles de cierta imprecisión. 
Este procedimiento es conocido bajo las siglas anglosajonas ADSA-D (Axisymmetric Drop Shape Analysis Diameter), es decir se añade una D de "diameter" (diámetro) para distinguirlo del anterior [12,13].

\subsection{Minimización de la Energía Superficial}

Un principio general en Física es que los sistemas evolucionan hacia su mínimo nivel de energía. En particular, este es el caso de las interfaces. La superficie de las gotas en equilibrio estático tiene la mínima energía. Por lo tanto, en el campo de las interfaces líquidas, se dice que una superficie mínima es una superficie que minimiza su área bajo algunas restricciones. La idea es minimizar la energía del sistema (superficial, gravitatoria, etc.) bajo algunas ligaduras como paredes, volumen fijo o presión constante.

De acuerdo con la definición de superficie mínima, la interfaz de una gota puede calcularse minimizando su energía superficial. Suponiendo que la gota no está apoyada en un substrato sólido, la energía superficial de todo el substrato sólido en contacto con el vapor es:

$$
U_{s v, 0}=\sigma_{\mathrm{sv}} A_{\mathrm{sv}, 0}
$$

donde $\sigma_{\mathrm{sv}}$ es la tensión superficial entre el sólido y el vapor, y $A_{\mathrm{sv}, 0}$ es el área de toda la superficie del substrato.

La energía superficial total $U_{T}$ de una gota sésil, viene dada por la suma de tres términos [3]: la energía de la superficie líquido-vapor $U_{\mathrm{lv}}$, la energía de la base líquidosólido $U_{\mathrm{sl}}$ y la energía superficial de la parte de la superficie del sólido $A_{\mathrm{sv}, 1}$ que está en contacto con el vapor $U_{\mathrm{sv}, 1}$ :

$$
U_{\mathrm{T}}=U_{\mathrm{lv}}+U_{\mathrm{sl}}+U_{\mathrm{sv}, 1}
$$

Por lo tanto, se obtiene:

$$
U_{\mathrm{T}}=\sigma_{\mathrm{Iv}} A_{\mathrm{lv}}+\iint_{\mathrm{sl}}\left(\sigma_{\mathrm{sl}}-\sigma_{\mathrm{sv}}\right) d A+U_{\mathrm{sv}, 0}
$$

donde la integral se calcula sobre la interfaz sólido-líquido. El último término de la derecha de la expresión (1.21) es constante y no influye en el cálculo del mínimo. 
Por tanto, es suficiente con minimizar:

$$
U=U_{\mathrm{T}}-U_{\mathrm{sv}, 0}=\sigma_{\mathrm{IV}} A_{\mathrm{lv}}+\iint_{\mathrm{sl}}\left(\sigma_{\mathrm{sl}}-\sigma_{\mathrm{sv}}\right) d A
$$

Teniendo en cuenta la ecuación de Young (1.18) esta expresión se puede reescribir como:

$$
U=\sigma_{\mathrm{IV}} A_{\mathrm{lv}}-\sigma_{\mathrm{lv}} \iint_{\mathrm{sl}} \cos \theta_{\mathrm{Y}} d A
$$

Los parámetros que intervienen en la ecuación (1.23) son $\theta_{Y}$ y $\sigma_{\mathrm{Iv}}$. El empleo de la ecuación de Young ha permitido suprimir la dependencia con las tensiones superficiales del sólido con el líquido o el vapor. Este hecho resulta de gran importancia puesto que tanto $\theta_{Y}$ como $\sigma_{\mathrm{lv}}$ pueden medirse con relativa facilidad. La minimización de la ecuación (1.23) constituye la base para el cálculo de la forma de una gota y es equivalente al uso de la ecuación de Young-Laplace (1.16).

\subsection{Gotas en superficies no homogéneas}

La ecuación de Young se obtiene para un sistema ideal con una superficie perfectamente homogénea y plana. Sin embargo, las superficies reales se apartan de dicha situación ideal, por lo que se hace necesario corregir la ecuación de Young con objeto de tener en cuenta las imperfecciones que surjan tanto de la rugosidad de la superficie como del hecho de que ésta no sea químicamente homogénea.

\subsubsection{Ley de Wenzel}

La ley de Wenzel [14] analiza el efecto de la rugosidad de una superficie sólida en el ángulo de contacto. Esta ley permite explicar ciertas observaciones experimentales que muestran cómo la rugosidad aumenta el carácter hidrófilo o hidrófobo del contacto. Si se denota $\theta^{*}$ al ángulo de contacto con la superficie rugosa y $\theta_{Y}$ al ángulo de contacto de Young con la superficie lisa, la Ley de Wenzel [14] establece que:

$$
\cos \theta^{*}=\hat{r} \cos \theta_{Y},
$$


donde $\hat{r}$ es la rugosidad. Por definición $\hat{r}>1$, lo que implica:

$$
\left|\cos \theta^{*}\right|>\left|\cos \theta_{Y}\right|
$$

Se puede deducir que:

$$
\left\{\begin{aligned}
\text { Si } \theta_{Y}<90^{\circ} & \text { (contacto hidrófilo }) \Rightarrow \theta^{*}<\theta_{Y} \Rightarrow \\
& \Rightarrow \text { el contacto es más hidrófilo debido a la rugosidad } \\
\text { Si } \theta_{Y}>90^{\circ} & \text { (contacto hidrófobo }) \Rightarrow \theta^{*}>\theta_{Y} \Rightarrow \\
& \Rightarrow \text { el contacto es más hidrófobo debido a la rugosidad }
\end{aligned}\right.
$$

Es importante destacar aquí que al establecer la ley de Wenzel se hace la suposición implícita de que la escala de la rugosidad de la superficie sólida se considera muy pequeña comparada con el tamaño de la gota [15]. En caso contrario no sería posible definir un único ángulo de contacto, la gota podría dejar de ser axisimétrica y la posición de la gota podría no ser estable. Es decir, al considerar un único ángulo de contacto en una superficie rugosa se supone que las moléculas del líquido están interaccionando macroscópicamente con una superficie plana, pero microscópicamente con una superficie rugosa.

\subsubsection{Ley de Cassie}

El mismo análisis de Wenzel puede realizarse para superficies sólidas químicamente no homogéneas. En esta situación también se consideran heterogeneidades de tamaño pequeño comparadas con las dimensiones del sistema con objeto de definir un único ángulo de contacto. Para simplificar, se analiza el caso de una pared sólida constituida por dos materiales diferentes. Si $\theta_{1}$ y $\theta_{2}$ son los ángulos de contacto de Young para cada material en el contacto microscópico y, $A_{1}$ y $A_{2}$ representan fracciones de superficie de los dos materiales $\left(A_{1}+A_{2}=1\right)$, entonces la relación de Cassie [16] es:

$$
\cos \theta^{*}=A_{1} \cos \theta_{1}+A_{2} \cos \theta_{2}
$$

La relación de Cassie pone de manifiesto que el coseno del ángulo de contacto en una superficie sólida microscópicamente inhomogénea es el baricentro del coseno de los ángulos de contacto de los diferentes componentes químicos de la superficie. 
La ley de Cassie explica ciertos resultados experimentales inesperados. Si no se tiene cuidado en el proceso de fabricación, una superficie puede presentar inhomogeneidades químicas y las propiedades de mojado pueden ser diferentes de las de la superficie homogénea. Por ejemplo, si una capa uniforme de teflón se deposita sobre un substrato rugoso, la superficie debería convertirse en hidrófoba. Pero si la capa es muy fina, el teflón depositado puede ser poroso y el recubrimiento no homogéneo; las propiedades de mojado se han modificado de acuerdo con la ley de Cassie y la ganancia en hidrofobicidad no sería tan alta como se podría esperar.

Las diversas imperfecciones que aparecen en superficies reales no sólo modifican el ángulo de contacto, como se ha visto brevemente en el caso de las leyes de Wenzel y de Cassie, sino que también dan lugar a la aparición de la histéresis del ángulo de contacto o histéresis angular que presentamos a continuación.

\subsection{Histéresis angular}

En sistemas reales no es común obtener un único ángulo de contacto estable como predice la ecuación de Young o las ecuaciones de Wenzel y de Cassie. En lugar de esto, para un determinado sistema se observa un intervalo de valores para los ángulos de contacto estables que se corresponden con estados metaestables de la energía libre del sistema. Como se verá más adelante, la existencia de este intervalo de valores da lugar a fenómenos de histéresis y por este motivo se denomina histéresis del ángulo de contacto. Los ángulos de contacto máximo y mínimo de este intervalo se denominan ángulo de avance $\left(\theta_{\mathrm{av}}\right)$ y ángulo de retroceso $\left(\theta_{\mathrm{re}}\right)$, debido a su aparición en situaciones en que la línea de contacto triple avanza o retrocede, respectivamente.

La histéresis del ángulo de contacto se manifiesta como una cierta resistencia al movimiento de la línea de contacto. Por ejemplo, la histéresis del ángulo de contacto da lugar a una resistencia a modificar la extensión de la superficie de contacto de una gota al variar su volumen. Así pues, al ir aumentando el volumen de la gota (en el avance), el ángulo de contacto es mayor de lo esperado, pues la extensión del líquido sobre la superficie es menor de la que correspondería para ese volumen. En el retroceso, cuando se disminuye su volumen, sucede lo contrario, la resistencia 
al movimiento de la línea de contacto permite alcanzar estados más extendidos de los que correspondería, esto implica que los ángulos de contacto son menores de lo esperado.

A menudo los ángulos de avance y retroceso presentan una gran reproducibilidad de manera que el intervalo de ángulos de contacto $\theta_{\mathrm{re}} \leq \theta \leq \theta_{\mathrm{av}}$, sustituye al valor único del ángulo de contacto $\theta_{Y}$ en la descripción de las propiedades de mojado de la superficie. La diferencia entre estos valores máximo y mínimo del ángulo de contacto sobre una superficie se conoce también con el nombre de histéresis del ángulo de contacto $\Delta \theta=\theta_{\mathrm{av}}-\theta_{\mathrm{re}}$. De este modo se cuantifica la importancia del fenómeno por esta diferencia de ángulos (longitud del intervalo). Cuando los ángulos de avance y retroceso son iguales decimos, de acuerdo a la definición anterior, que la histéresis es cero o que no hay histéresis del ángulo de contacto.

Para la medida de estos ángulos de avance y retroceso se realizan ciclos de histéresis. Los ciclos de histéresis con gotas sésiles se llevan a cabo inyectando líquido para aumentar el volumen de la gota (avance) y succionando posteriormente dicho líquido para disminuir su volumen (retroceso).

Prácticamente en todas las superficies solidas se produce histéresis del ángulo de contacto, y a pesar de que éste es un fenómeno que ha sido estudiado extensamente en las últimas décadas, las causas subyacentes y su origen no se comprenden en su totalidad.

A continuación se presenta brevemente la herramienta que se ha utilizado para realizar las simulaciones de procesos de histéresis en gotas sésiles, el software libre Surface Evolver.

\subsection{Gotas sésiles con Surface Evolver}

La forma de las gotas sésiles sobre superficies reales es muy variada y depende fuertemente de las características de dichas superficies. Encontrar la forma de la gota se reduce a minimizar la energía del sistema $U$, para lo que es necesario utilizar herramientas que permitan llevar a cabo dicha minimización. En este trabajo se hace uso de un software diseñado para este propósito llamado Surface Evolver [6]. 


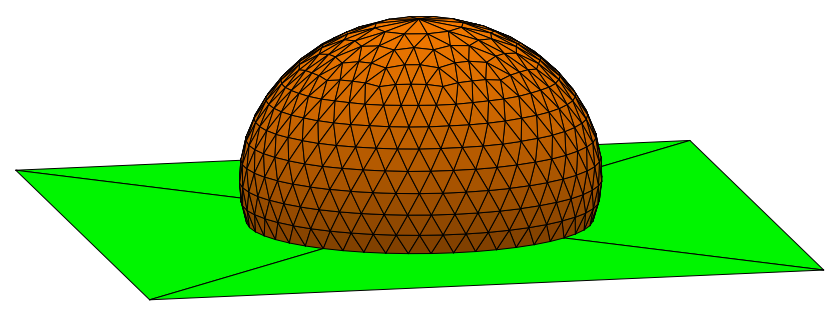

Figura 1.6: Imagen de un momento de la evolución de una gota durante la simulación en Surface Evolver.

Para la realización de estos cálculos se han desarrollado un conjunto de rutinas y secuencias de instrucciones que permiten junto con este software, determinar la forma de las gotas depositadas sobre superficies caracterizadas por distintos patrones. Estas simulaciones ${ }^{2}$ permiten no sólo conocer detalladamente la forma de la superficie de las gotas, sino también los parámetros característicos del mojado de superficies como el área de contacto, los ángulos de contacto, etc.

En este apartado se presenta brevemente dicho programa, junto con su aplicación para el análisis de la forma de las gotas sésiles.

\subsubsection{Surface Evolver}

Surface Evolver es un programa informático destinado al modelado de la forma de superficies sometidas a diferentes fuerzas o ligaduras. Este software ha sido diseñado y desarrollado por Ken Brakke del Departamento de Matemáticas de la Universidad de Susquehanna en Pennsylvania (Estados Unidos) y es de dominio público.

Surface Evolver minimiza la energía de un sistema de acuerdo con las ligaduras a las está sometido. Utiliza el método de elementos finitos en el cual la superficie se representa por una teselación triangular, en la que los elementos que intervienen en su construcción son vértices, aristas y caras (ver figura 1.6). Se pueden encontrar en la literatura varios trabajos demostrando el uso de Surface Evolver en el campo de

\footnotetext{
${ }^{2} \mathrm{Si}$ bien estos cálculos numéricos, no hacen uso de una semilla de aleatoriedad, ni pretenden describir dinámicamente un proceso real, por su similitud en la evolución del sistema hasta su valor de equilibrio, imitando una gota que se extiende sobre una superficie, usamos familiarmente el nombre de simulaciones. Aunque este término esté frecuentemente reservado para aquéllos.
} 
los fenómenos interfaciales de los fluidos [3, 8, 17-29].

El software parte de un archivo que contiene una superficie inicial y las instrucciones para el cálculo de la energía del sistema. Luego, de manera manual o automatizada (mediante secuencias de instrucciones), se dan las órdenes al programa para el proceso de minimización de la energía del sistema. Se muestra un ejemplo sencillo en la figura 1.7.

La superficie de partida se describe en bloques correspondientes a cuatro elementos; estos son vértices, aristas, caras y cuerpos:

1. Se enumeran los vértices (puntos que componen la triangulación) indicando sus coordenadas $x, y, z$. [Líneas 16 a 24 en la figura 1.7]

2. Se enumeran las aristas que han de formar los vértices mencionados anteriormente, indicando los números identificativos de los vértices de origen y fin. [Líneas 27 a 39 en el ejemplo]

3. Se enumeran las caras que han de formar las aristas anteriores, indicando el número identificativo acompañado de un signo negativo para la orientación de la arista si fuese necesario. Nótese que no es imprescindible que se trate de triángulos. Surface Evolver transformará los polígonos en triángulos añadiendo los vértices y aristas convenientes. [Líneas 42 a 47 en el ejemplo]

4. Se enumeran los distintos cuerpos que forman estas caras indicando de nuevo sus números identificativos y orientación de dichas caras. [Líneas 50 a 51 en el ejemplo]

Estos elementos pueden estar sujetos a ligaduras. [Líneas 8 y 9]

Las energías pueden asociarse a estos elementos de manera directa o a través de cálculos a partir de sus características.

El software trae implementada la posibilidad de incluir el campo gravitatorio con sencillez, atribuyendo una densidad a cada cuerpo [línea 51] y fijando un valor de la aceleración debida a la gravedad [línea 5]. Del mismo modo, incorpora la posibilidad de atribuir una densidad de energía a caras y aristas que pueden utilizarse para incluir energías interfaciales y de línea. 
Las caras tienen una densidad de energía 1 por defecto, por ejemplo, la línea

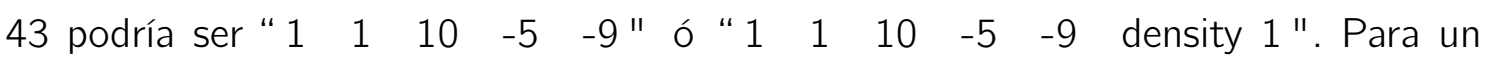
caso que asocia energía a líneas para el cálculo de la energía superficial del contacto véanse las líneas 8 y 11 a 13.

\subsubsection{Energía en Surface Evolver}

Sea una gota de un líquido en equilibrio con su vapor depositada sobre una superficie que se conoce tanto topológica como energéticamente. El volumen de la gota es $V$, las densidades del líquido y su vapor son $\rho_{\mathrm{l}}$ y $\rho_{\mathrm{v}}$ respectivamente, y las tres tensiones superficiales son $\sigma_{\mathrm{lv}}, \sigma_{\mathrm{sl}}$ y $\sigma_{\mathrm{sv}}$.

Encontrar la forma de la gota se reduce a minimizar la energía del sistema $U$ a volumen constante, considerando el líquido incompresible (es decir, no varía su densidad). Para ello variamos la forma de la interfaz líquido-vapor y la extensión de la superficie de contacto (interfaces sólido-líquido y sólido-vapor) hasta alcanzar un mínimo de energía del sistema.

La energía de una gota sésil de volumen fijo bajo la acción de la gravedad viene dada por la expresión

$$
U=\iint_{\mid \mathrm{V}} \sigma_{\mathrm{IV}} d A+\iint_{\mathrm{s} \mid}\left(\sigma_{\mathrm{sl}}-\sigma_{\mathrm{sV}}\right) d A+\iiint_{\mathrm{I}}\left(\rho_{\mathrm{l}}-\rho_{\mathrm{V}}\right) g z d V
$$

donde las integrales se realizan sobre la interfaz líquido-vapor Iv, la interfaz sólidolíquido sl y el volumen del líquido, respectivamente; $g$ es la aceleración debida a la gravedad y $z$ la coordenada vertical de la gota.

Dividiendo por la tensión interfacial líquido-vapor $\sigma_{\mathrm{lv}}$ se tiene

$$
\varepsilon=\frac{U}{\sigma_{\mathrm{lv}}}=\iint_{\mathrm{lv}} d A+\iint_{\mathrm{sl}} \frac{\left(\sigma_{\mathrm{sl}}-\sigma_{\mathrm{sv}}\right)}{\sigma_{\mathrm{lv}}} d A+\iiint_{\mathrm{l}} \frac{\left(\rho_{\mathrm{l}}-\rho_{\mathrm{v}}\right) g z}{\sigma_{\mathrm{lv}}} d V,
$$

que puede escribirse de manera más sencilla como:

$$
\varepsilon=\iint_{\mathrm{IV}} d A-\iint_{\mathrm{sl}} \cos \theta_{Y} d A+\iiint_{\mathrm{I}} \frac{Z}{l_{0}^{2}} d V
$$

donde se ha hecho uso de la ecuación de Young (1.18) y de la longitud de capilaridad Io (1.14). 


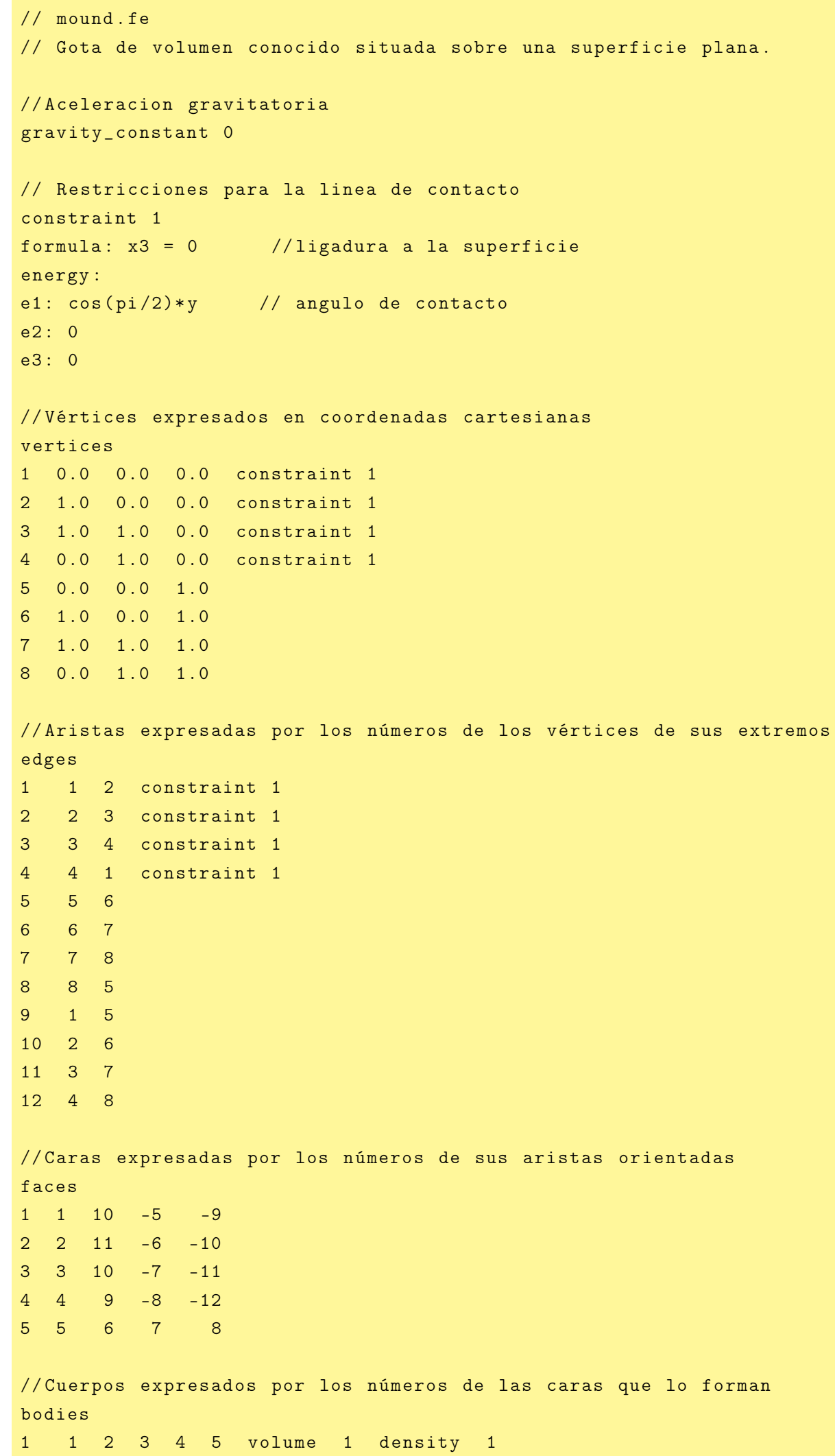


Para implementar la anterior ecuación en Surface Evolver es preciso expresarla en términos de cantidades adimensionales. Puesto que $\varepsilon$ tiene unidades de longitud al cuadrado y el resto de magnitudes en (1.29) tienen dimensiones de longitud, superficie o volumen, es suficiente con normalizar en términos del volumen de la gota $V_{\mathrm{g}}$ :

$$
\widetilde{\varepsilon}=\frac{U}{\sigma_{\mathrm{IV}} V_{\mathrm{g}}^{2 / 3}}=\iint_{\mathrm{IV}} \frac{d A}{V_{\mathrm{g}}^{2 / 3}}-\iint_{\mathrm{s}} \cos \theta_{\mathrm{Y}} \frac{d A}{V_{\mathrm{g}}^{2 / 3}}+\iiint_{\mathrm{l}} \frac{V_{\mathrm{g}}^{2 / 3}}{l_{0}^{2}} \frac{Z}{V_{\mathrm{g}}^{1 / 3}} \frac{d V}{V_{\mathrm{g}}},
$$

es decir

$$
\widetilde{\varepsilon}=\iint_{\mid V} d \widetilde{A}-\iint_{\text {sl }} \cos \theta_{Y} d \widetilde{A}+\iiint_{\mid} \frac{\widetilde{z}}{\widetilde{l}_{0}^{2}} d \widetilde{V},
$$

donde las magnitudes con tilde $(\sim)$ son adimensionales, y además se verifica

$$
\iiint_{1} d \widetilde{V}=1
$$

La implementación en Surface Evolver es la siguiente:

\section{Términos superficiales}

1. Interfaz líquido-vapor

$$
\iint_{\mathrm{IV}} d \widetilde{A}
$$

Se toma el valor 1 para la densidad de energía superficial de la interfaz líquidovapor.

2. Interfaz sólido-líquido

$$
\iint_{\text {sl }}\left(-\cos \theta_{Y}\right) d \tilde{A}
$$

En lugar de calcular esta integral de superficie, es posible reescribir la ecuación (1.34) en términos de una integral de línea. Para ello se hace uso del teorema del rotacional:

$$
\iint_{A}(\nabla \times \mathbf{F}) \cdot d \mathbf{A}=\int_{\partial A} \mathbf{F} \cdot d \mathbf{L}
$$


de modo que se puede sustituir este término por la circulación del vector $\mathbf{F}$ :

$$
\iint_{\mathrm{sl}}\left(-\cos \theta_{\mathrm{Y}}\right) d \widetilde{A}=\iint_{\mathrm{s} l}\left(-\cos \theta_{Y}\right) \mathbf{n} \cdot d \widetilde{\mathbf{A}}=\int_{\mathrm{slV}} \mathbf{F} \cdot d \widetilde{\mathbf{L}} .
$$

$\operatorname{con} \nabla \times \mathbf{F}=-\cos \theta_{Y} \mathbf{n}$, siendo $\mathbf{n}$ un vector unitario normal a la superficie.

\section{Término de volumen}

1. La contribución gravitatoria viene dada por la siguiente integral de volumen

$$
\iiint_{l} \frac{\widetilde{z}}{\widetilde{l}_{0}^{2}} d \widetilde{V}
$$

La diferencia de densidades líquido-vapor se puede considerar 1 en Surface Evolver y atribuir su contribución al término $1 / \widetilde{I}_{0}^{2}$.

En capítulos sucesivos se expondrán aplicaciones de esta herramienta a diferentes situaciones. 


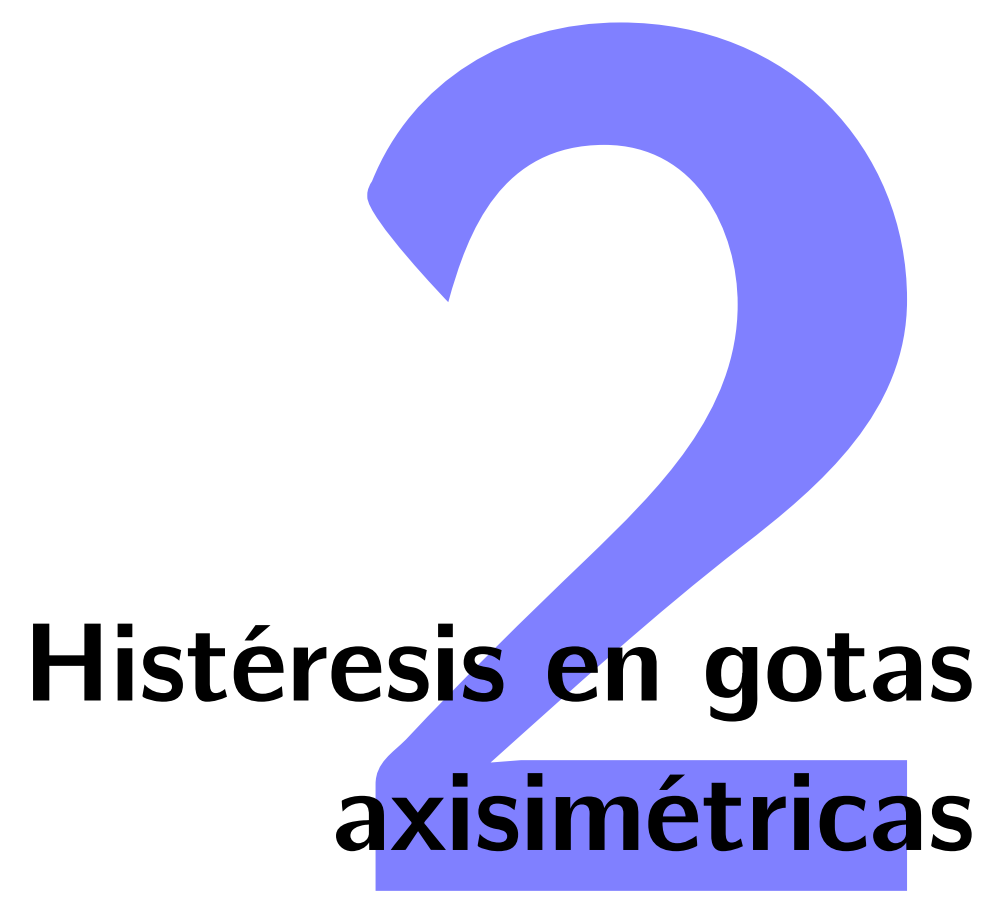

\subsection{Introducción}

Comprender la forma de una gota de líquido en equilibrio mecánico sobre una superficie plana es importante tanto para la Ciencia de Superficies como para muchas aplicaciones en Ingeniería e Industria. Una clave para la determinación de esta forma es el análisis del ángulo de contacto de la gota líquida con la superficie. Es bien conocido que este ángulo de contacto se puede obtener del balance entre la tensión superficial del líquido-vapor $\sigma_{\mathrm{Iv}}$, la tensión superficial del sólido-vapor $\sigma_{\mathrm{sv}}$ y la tensión superficial del líquido-sólido $\sigma_{\mathrm{Is}}$. Como se ha visto en el capítulo anterior, este balance de las fuerzas superficiales viene dado por la ecuación de Young (1.18). Esta ecuación es válida para sistemas ideales en los cuales la superficie es lisa, homogénea y rígida y existe, por lo tanto, un único estado de equilibrio caracterizado por un único ángulo de contacto.

La situación es bastante diferente en sistemas reales pues prácticamente todas las superficies sólidas son rugosas y químicamente heterogéneas en mayor o menor grado. Esto implica que $\theta_{Y}$ no es, en general, igual al ángulo de contacto aparente [30] medido en un experimento. Consecuentemente, la aplicación directa de la ecuación 
de Young no es válida en sistemas reales y se deben recurrir a modificaciones como las ecuaciones de Cassie para substratos químicamente heterogéneos [16], o la de Wenzel [14] para superficies rugosas. Además, debido a la rugosidad y a la heterogeneidad química, la mayoría de los sistema reales exhiben histéresis del ángulo de contacto en el cual hay un rango de ángulos de contacto aparente prácticamente estables comprendidos entre un límite inferior, el ángulo de contacto de retroceso $\theta_{\text {re }}$ y un límite superior dado por el ángulo de contacto de avance $\theta_{\mathrm{av}}$.

En el pasado se han realizado varios intentos dirigidos a estudiar gotas con histéresis del ángulo de contacto de un modo semi-empírico, sin entrar en los detalles de su origen microscópico. En este contexto, en el trabajo pionero de Adam and Jessop [31] se incluye una fuerza externa en la ecuación de Young (1.18) de modo que se tiene

$$
\sigma_{\mathrm{sv}}-\sigma_{\mathrm{ls}}=\sigma_{\mathrm{lv}} \cos \theta_{\mathrm{av}}+f
$$

para la situación de avance, y

$$
\sigma_{\mathrm{sv}}-\sigma_{\mathrm{Is}}=\sigma_{\mathrm{lv}} \cos \theta_{\mathrm{re}}-f
$$

para la situación de retroceso. En las ecuaciones (2.1) y (2.2) $f$ es una fuerza de fricción por unidad de longitud, actuando sobre la línea de contacto triple con igual intensidad para los ángulos de avance y retroceso. Algunos trabajos recientes [32-34] han estudiado la histéresis del ángulo de contacto para gotas sobre una superficie inclinada considerando una línea de contacto triple circular como una aproximación válida para pequeñas gotas o bajas inclinaciones (téngase en cuenta que cuando se considera una línea de contacto triple fija, se está suponiendo de forma implícita una fuerza de fricción estática). Dussan y Chow [35,36] también estudiaron gotas sobre superficies inclinadas pero considerando gotas elongadas con una línea de contacto formada por dos arcos semicirculares unidos por dos lados paralelos a lo largo de la dirección de inclinación. Una estrategia de simulación de elementos finitos en la cual se permite que se mueva la línea de contacto triple fue propuesta por Illiev $[37,38]$ considerando un término de retención proporcional al área barrida por el movimiento virtual de la línea de contacto triple. Un enfoque basado en un problema de optimización fue llevado a cabo por Dimitrakopoulos y Higdon [39] buscando sobre todas 
las líneas de contacto con ángulos de contacto locales $\theta_{\mathrm{n}}$ tales que $\theta_{\mathrm{re}} \leq \theta_{\mathrm{n}} \leq \theta_{\mathrm{av}}$.

En el espíritu de estos trabajos, el objetivo del presente capítulo es proponer un nuevo tratamiento de la histéresis del ángulo de contacto estática, suponiendo una fuerza de fricción empírica por unidad de longitud de la línea de contacto, de modo que ésta se mantiene fija para ángulos de contacto comprendidos entre los de avance y retroceso $\theta_{\mathrm{re}}<\theta<\theta_{\mathrm{av}}$ y se mueve para impedir ángulos fuera de dicho intervalo. En este sentido, es de destacar que desde un punto de vista microscópico se puede suponer que la fuerza de fricción se debe a las fuerzas de anclaje ejercidas por los defectos de la superficie sobre la línea de contacto, como han expuesto Joanny y de Gennes [40, 41].

Este capítulo está estructurado como sigue. En primer lugar se realiza un tratamiento del problema que solo es válido para gotas axisimétricas, basado en resolver la ecuación de Young-Laplace en coordenadas cilíndricas. A continuación se propone un estudio a partir del programa Surface Evolver [42] en el que se realiza una modificación que permite incorporar una fuerza de fricción en la búsqueda de la forma estable de la gota de líquido. Este procedimiento, que es también adecuado para estudiar gotas no-axisimétricas, permite diseñar un nuevo método para medir ángulos de contacto en Surface Evolver. Finalmente se comparan los resultados de estas aproximaciones con datos experimentales.

\subsection{Solución de la ecuación de Young-Laplace para gotas axisimétricas}

Como ya se ha expuesto en el capítulo 1 , la ecuación de Young-Laplace $[1,2]$ establece que la diferencia de presión $\Delta P$ a lo largo de la interfaz de un fluido en equilibrio es proporcional a la tensión superficial y a la curvatura media. En el subapartado 1.3.3 vimos que la ecuación de Young-Laplace en gravedad viene dada por la ecuación (1.16), que puede reescribirse como

$$
\mathcal{H}_{\Sigma}(u, v)=b-\frac{1}{2} c_{1} h(u, v)
$$


siendo $b=1 / r_{0}$ la curvatura del ápice de la gota y $c_{1}=1 / l_{0}^{2}$ la constante de capilaridad, donde $r_{0}$ es el radio de curvatura en el ápice, y $I_{0}$ la longitud de capilaridad.

En general, la expresión (2.3) es una ecuación no lineal en derivadas parciales difícil de resolver. Sin embargo, para simetría cilíndrica, se transforma en un sistema de ecuaciones diferenciales ordinarias que puede expresarse en términos de la longitud de arco $s$ del perfil de la gota, tal y como se muestra en el Apéndice A. Es de destacar que este sistema de ecuaciones diferenciales es el punto de partida del método ADSA (Axisimetric Drop Size Analysis [9]).

La solución de la ecuación de Young-Laplace para una gota sésil axisimétrica permite obtener el ángulo tangencial del perfil de la gota $\theta\left(s, b, c_{l}\right)$, la coordenada axial $z\left(s, b, c_{1}\right)$, la coordenada radial $R\left(s, b, c_{1}\right)$, el área de la interfaz líquido-vapor $A\left(s, b, c_{1}\right)$, y el volumen $V\left(s, b, c_{1}\right)$ en función de la longitud de arco $s$, la curvatura del ápice de la gota $b$ y la constante de capilaridad $c_{1}$.

Si se conocen el volumen $V$ y el ángulo de contacto $\theta$ de una gota de líquido con constante de capilaridad $c_{1}$, se puede obtener la curvatura del ápice $b$ y la longitud de arco en la línea de contacto triple $s_{c}$ resolviendo el sistema de ecuaciones:

$$
V\left(s_{c}, b, c_{1}\right)=V ; \quad \theta\left(s_{c}, b, c_{1}\right)=\theta
$$

Se pueden obtener entonces otras cantidades de interés. Por ejemplo, la altura de la gota viene dada por $H=z\left(s_{c}, b, c_{1}\right)$ y el radio de la línea de contacto triple viene dado por $R=R\left(s_{c}, b, c_{1}\right)$. A la inversa, si se sabe que la gota tiene un volumen $V$ y el radio de la línea de contacto es $R$ se debe resolver:

$$
V\left(s_{c}, b, c_{1}\right)=V ; \quad R\left(s_{c}, b, c_{l}\right)=R
$$

para obtener $b$ y $s_{c}$, a partir de las cuales se puede calcular el ángulo de contacto $\theta$. Es de destacar que este método es algo diferente del método ADSA-D en el cual se considera el diámetro máximo D [9].

Se considera a continuación un experimento para determinar los ángulos de contacto de avance y retroceso de una gota sésil a partir de un ciclo de ángulos de contacto (DOCA, dynamic one-cycle contact angle [43]). Sobre una superficie horizontal se deposita una gota líquida con un volumen inicial $V_{\text {ini. }}$ Se supone que la 
gota tiene un ángulo de contacto inicial $\theta_{\text {ini }}$ en el intervalo $\theta_{\text {re }} \leq \theta_{\text {ini }} \leq \theta_{\text {av }}$. Se aumenta lentamente el volumen de la gota, a velocidad constante, hasta que alcanza un volumen final $V_{\text {fin }}$ y entonces, se disminuye éste, a la misma velocidad, hasta el volumen inicial $V_{\text {ini. }}$. Durante el proceso de crecimiento se observa que la línea de contacto triple se mantiene fija ( $R=$ constante) hasta que se alcanza el ángulo de contacto de avance $\theta_{\mathrm{av}}$, entonces la línea de contacto se mueve ( $R$ crece) mientras que el ángulo de contacto, denominado ángulo de contacto de avance, permanece constante $\left(\theta=\theta_{\mathrm{av}}=\right.$ cte. $)$ hasta que se para el crecimiento de la gota $\left(V=V_{\text {fin }}\right)$. Al comienzo del proceso de reducción de volumen, la línea de contacto triple se mantiene fija mientras que el ángulo de contacto disminuye hasta que alcanza su valor de retroceso y el radio de la línea de contacto triple comienza a disminuir.

Merece la pena destacar que la mayoría de los sistemas líquido-sólido presentan ángulos de contacto de retroceso dependientes del tiempo [43], pero estas situaciones son debidas a cambios en el sólido y, por lo tanto, una descripción microscópica adecuada está fuera de los objetivos de este trabajo. En el subapartado 2.2.1 se presentará un tratamiento semi-fenomenológico para sistemas de este tipo. No obstante, se han observado ángulos de contacto de retroceso constantes en algunos sistemas líquido-sólido de superficies biodegradables, como agua sobre superficies de silicio cubiertas de ácido poliláctico $[43,44]$.

La descripción de un experimento DOCA con las ecuaciones (2.4) y (2.5) es directa:

1. Gota inicial: El volumen inicial $V_{\text {ini }}$ y el ángulo de contacto inicial $\theta_{\text {ini }}$ son conocidos. Utilizando la ecuación (2.4) se determinan $b$ y $s_{c}$ a partir de los cuales se obtiene el radio inicial $R_{\text {ini }}$.

2. Crecimiento de la gota mientras $\theta<\theta_{\mathrm{av}}$ : En esta situación se conocen el volumen $V>V_{\text {ini }}$ y el radio $R=R_{\text {ini }}$. Utilizando la ecuación (2.5) se determinan $b$ y $s_{c}$ y a partir de ellos se obtiene el ángulo de contacto. Cuando $\theta$ alcanza el valor $\theta_{\mathrm{av}}$ el sistema llega a una situación de avance.

3. Situación de avance: El volumen $V$ y el ángulo de contacto $\theta=\theta_{\mathrm{av}}$ son conocidos. El radio de la línea de contacto triple $R$ se calcula utilizando la ecuación 
(2.4). El máximo valor del radio $R_{\max }$ se obtiene cuando $V=V_{\text {fin }}$.

4. Reducción de la gota mientras $\theta>\theta_{\text {re }}$ : En esta situación el volumen $V<V_{\text {fin }}$ y el radio $R=R_{\max }$ son conocidos. Utilizando la ecuación (2.5) se determina el ángulo de contacto. Cuando $\theta$ llega a $\theta_{\text {re }}$ el sistema alcanza la situación de retroceso.

5. Situación de retroceso: El volumen $V$ y el ángulo de contacto $\theta=\theta_{\text {re }}$ son conocidos. El radio de la línea de contacto triple $R$ se calcula utilizando la ecuación (2.4). Cuando $V=V_{\text {ini }}$ los cálculos terminan.

La figura 2.1 muestra el diagrama de flujo del algoritmo utilizado para obtener un ciclo de histéresis de una gota de líquido, resolviendo la ecuación de Young-Laplace.

La cuestión que se plantea a continuación es cómo expresar este esquema sencillo de los experimentos DOCA en términos de una fuerza de fricción actuando sobre la línea de contacto triple. La respuesta a esta pregunta se obtiene considerando un enfoque similar al de las ecuaciones (2.1) y (2.2) de Adam y Jessop [31] pero con valores diferentes de la fuerza de fricción por unidad de longitud, dependiendo de la situación. Así, se tiene:

$$
\sigma_{\mathrm{sv}}-\sigma_{\mathrm{ls}}=\sigma_{\mathrm{lv}} \cos \theta_{\mathrm{av}}+f_{\mathrm{av}}
$$

para una gota axisimétrica en situación de avance, y

$$
\sigma_{\mathrm{sv}}-\sigma_{\mathrm{ls}}=\sigma_{\mathrm{lv}} \cos \theta_{\mathrm{re}}-f_{\mathrm{re}}
$$

para una gota axisimétrica en situación de retroceso. Comparando ambas con la ecuación de Young (1.18) se obtiene:

$$
f_{\mathrm{av}}=\sigma_{\mathrm{lv}}\left(\cos \theta_{Y}-\cos \theta_{\mathrm{av}}\right)
$$

y

$$
f_{\mathrm{re}}=\sigma_{\mathrm{lv}}\left(\cos \theta_{\mathrm{re}}-\cos \theta_{Y}\right) .
$$

Conviene destacar aquí que la expresión habitual para el ángulo de contacto de Young,

$$
\theta_{\mathrm{Y}}=\frac{\theta_{\mathrm{re}}+\theta_{\mathrm{av}}}{2},
$$




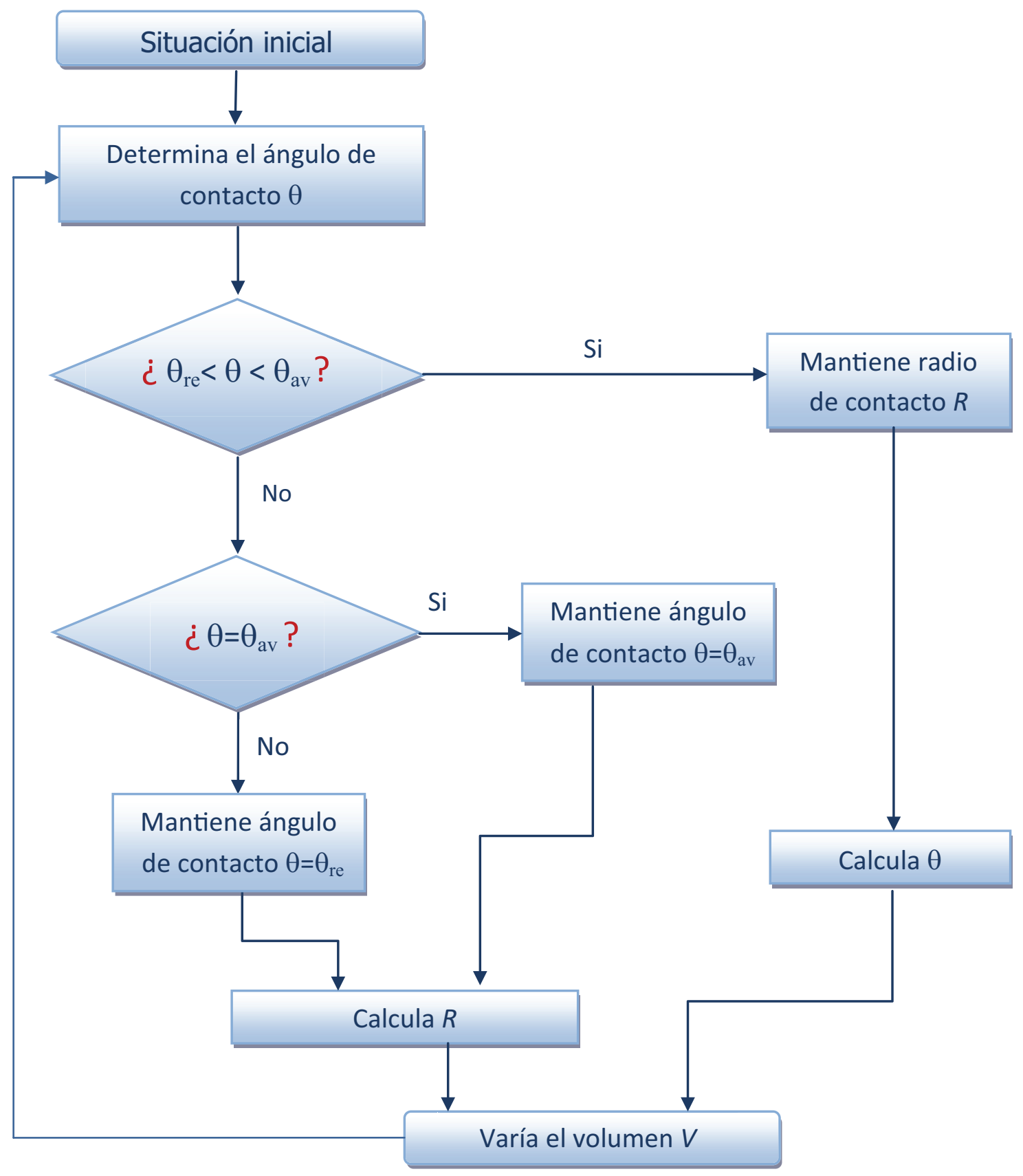

Figura 2.1: Diagrama de flujo del algoritmo utilizado para obtener un ciclo de histéresis de una gota de líquido, resolviendo la ecuación de Young-Laplace en coordenadas cilíndricas. 
implica que $f_{\mathrm{re}} \neq f_{\mathrm{av}}$. Recientes experimentos demuestran que esta suposición es cercana al ángulo de contacto más estable de la gota $[45,46]$. Sin embargo, si se considera $f_{\mathrm{re}}=f_{\mathrm{av}}$ como en las ecuaciones (2.1) y (2.2), se obtiene una expresión ligeramente diferente pero también común para el ángulo de contacto de Young

$$
\theta_{Y}=\arccos \left(\frac{\cos \theta_{\mathrm{re}}+\cos \theta_{\mathrm{av}}}{2}\right) \text {. }
$$

Otras opciones son posibles para $\theta_{Y}$, como la propuesta formulada por Tadmor [47] teniendo en cuenta la energía de línea asociada a la línea de contacto triple.

El hecho de que la línea de contacto triple esté fija para ángulos de contacto $\theta$ pertenecientes al intervalo $\theta_{\mathrm{re}}<\theta<\theta_{\mathrm{av}}$ se debe a la aparición de una fuerza de fricción estática por unidad de longitud $f_{\theta}$. En este caso se tiene

$$
\sigma_{\mathrm{sv}}-\sigma_{\mathrm{ls}}=\sigma_{\mathrm{lv}} \cos \theta-f_{\theta}
$$

con

$$
f_{\theta}=\sigma_{\mathrm{lv}}\left(\cos \theta-\cos \theta_{Y}\right) \text { si } \theta_{\mathrm{re}}<\theta<\theta_{\mathrm{av}} \text {. }
$$

\subsubsection{Simulación de un ciclo de histéresis}

En la figura 2.2 se muestran los resultados de la simulación de un ciclo de histéresis obtenidos siguiendo el algoritmo de la figura 2.1 y resolviendo la ecuación de YoungLaplace (2.3) en coordenadas cilíndricas. La figura 2.2a recoge la evolución temporal del volumen; la figura 2.2b la evolución temporal del radio de contacto; y la figura 2.2c la evolución temporal del ángulo de contacto. En dicha figura se ha considerado un volumen inicial $V_{\text {ini }}=0,030 \mathrm{~cm}^{3}$ y un volumen final $V_{\text {fin }}=0,118 \mathrm{~cm}^{3}$ de agua depositada sobre una superficie de silicio cubierta de ácido poliláctico para el cual $\theta_{\mathrm{av}}=(79,82 \pm 0,28)^{\circ}$ y $\theta_{\mathrm{re}}=(60,97 \pm 0,31)^{\circ}$ [44]. La longitud de capilaridad es $I_{0}=2,72 \mathrm{~mm}$ y se ha supuesto que el ángulo de contacto de Young toma el valor $\theta_{Y}=\left(\theta_{\text {rec }}+\theta_{\mathrm{av}}\right) / 2=70,40^{\circ}$. Para simplificar se ha considerado que el ángulo de contacto inicial $\theta_{i n i}$ es igual a $\theta_{Y}$. Es de destacar que para obtener los resultados presentados en la figura 2.2 se ha aumentado el volumen desde $V_{\text {ini }}$ a $V_{\text {fin }}$ a velocidad constante y entonces, se ha disminuido de nuevo hasta $V_{\text {ini }}$. 

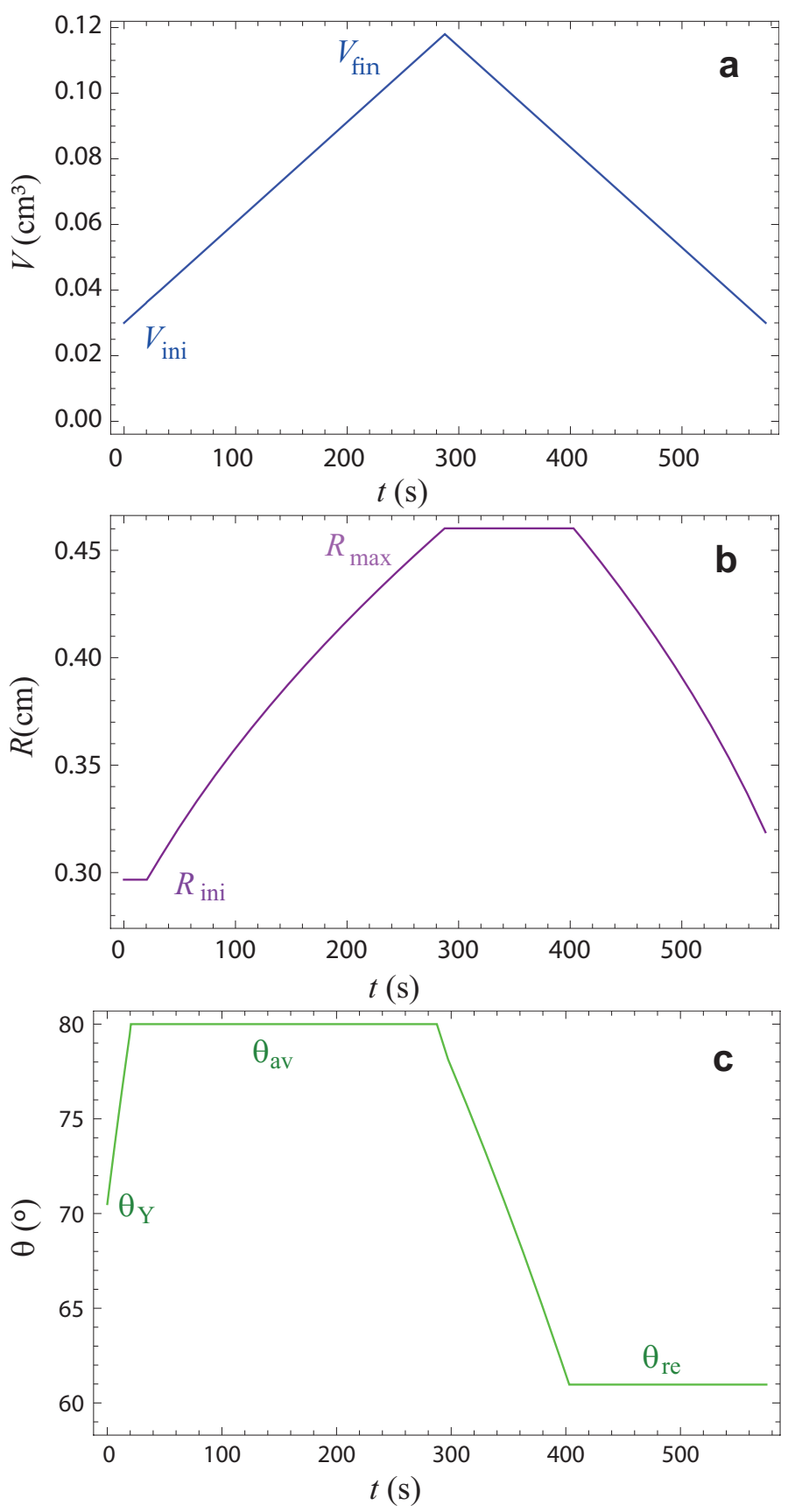

Figura 2.2: Ciclo de histéresis de una gota de agua sobre una superficie de silicio recubierta de ácido poliláctico, obtenido resolviendo la ecuación de Young-Laplace (2.3) en coordenadas cilíndricas, entre $V_{\text {ini }}=0,03 \mathrm{~cm}^{3}$ y $V_{\text {fin }}=0,118 \mathrm{~cm}^{3}$. a) Evolución temporal del volumen. b) Evolución temporal del radio de contacto. c) Evolución temporal del ángulo de contacto. 
Con el fin de obtener el ciclo de histéresis completo, se han considerado las cuatro fases siguientes:

1. En la primera fase, al aumentar el volumen desde $V_{\text {ini }}=0,03 \mathrm{~cm}^{3}$ hasta $V_{\text {ca }}=$ $0,036 \mathrm{~cm}^{3}$ se mantiene el radio de contacto constante $R_{\text {ini }}=0,2967 \mathrm{~cm}$, y el ángulo de contacto va aumentando a partir de $\theta_{Y}=70,4^{\circ}$.

2. En la segunda fase, el volumen aumenta desde $V_{\text {ca }}=0,036 \mathrm{~cm}^{3}$ hasta $V_{\text {fin }}=$ $0,118 \mathrm{~cm}^{3}$ y el ángulo de contacto permanece constante e igual al ángulo de contacto de avance $\theta_{\mathrm{av}}=79,82^{\circ}$, mientras aumenta el radio de contacto.

3. En la tercera fase, el volumen disminuye desde $V_{\text {fin }}=0,118 \mathrm{~cm}^{3}$ hasta $V_{\text {cr }}=$ $0,0826 \mathrm{~cm}^{3}$ y se mantiene el radio de contacto constante $R_{\max }=0,4602 \mathrm{~cm}$, disminuyendo el ángulo de contacto.

4. En la cuarta fase, el volumen disminuye desde $V_{\text {cr }}=0,0826 \mathrm{~cm}^{3}$ hasta $V_{\text {ini }}=$ $0,03 \mathrm{~cm}^{3}$ y el ángulo de contacto permanece constante e igual al ángulo de contacto de retroceso $\theta_{\text {re }}=60,97^{\circ}$, mientras disminuye el radio de contacto.

La figura 2.3 considera el mismo sistema de la figura 2.2 pero representando los resultados en función del volumen de la gota. En la figura 2.3a se representan los ángulos de contacto medios mientras que en la figura $2.3 \mathrm{~b}$ se presenta el radio de la línea de contacto triple. La figura 2.3c muestra la fuerza de fricción adimensional $f_{\theta} / \sigma_{\mathrm{Iv}}$ en función del volumen. El máximo valor de $f_{\theta}$ es $f_{\mathrm{re}}$ mientras que el valor mínimo es $-f_{\mathrm{av}}$. El valor negativo de $f_{\theta}$ indica simplemente que la fuerza de fricción está dirigida hacia el centro de la circunferencia de la línea de contacto triple, opuesta a la dirección de avance. Análogamente, los valores positivos de $f_{\theta}$ indican que la fuerza de fricción está dirigida hacia fuera del centro de la circunferencia correspondiente a la línea de contacto triple, opuesta ahora a la dirección de retroceso.

La técnica presentada en este apartado, es válida únicamente para gotas axisimétricas para las cuales la simetría cilíndrica juega un papel esencial. Para gotas no axisimétricas la ecuación de Young-Laplace no puede expresarse como un conjunto de ecuaciones diferenciales ordinarias. Además, en lugar de tener un único ángulo de contacto $\theta$, en general el sistema tiene ángulos de contacto que dependen de la 

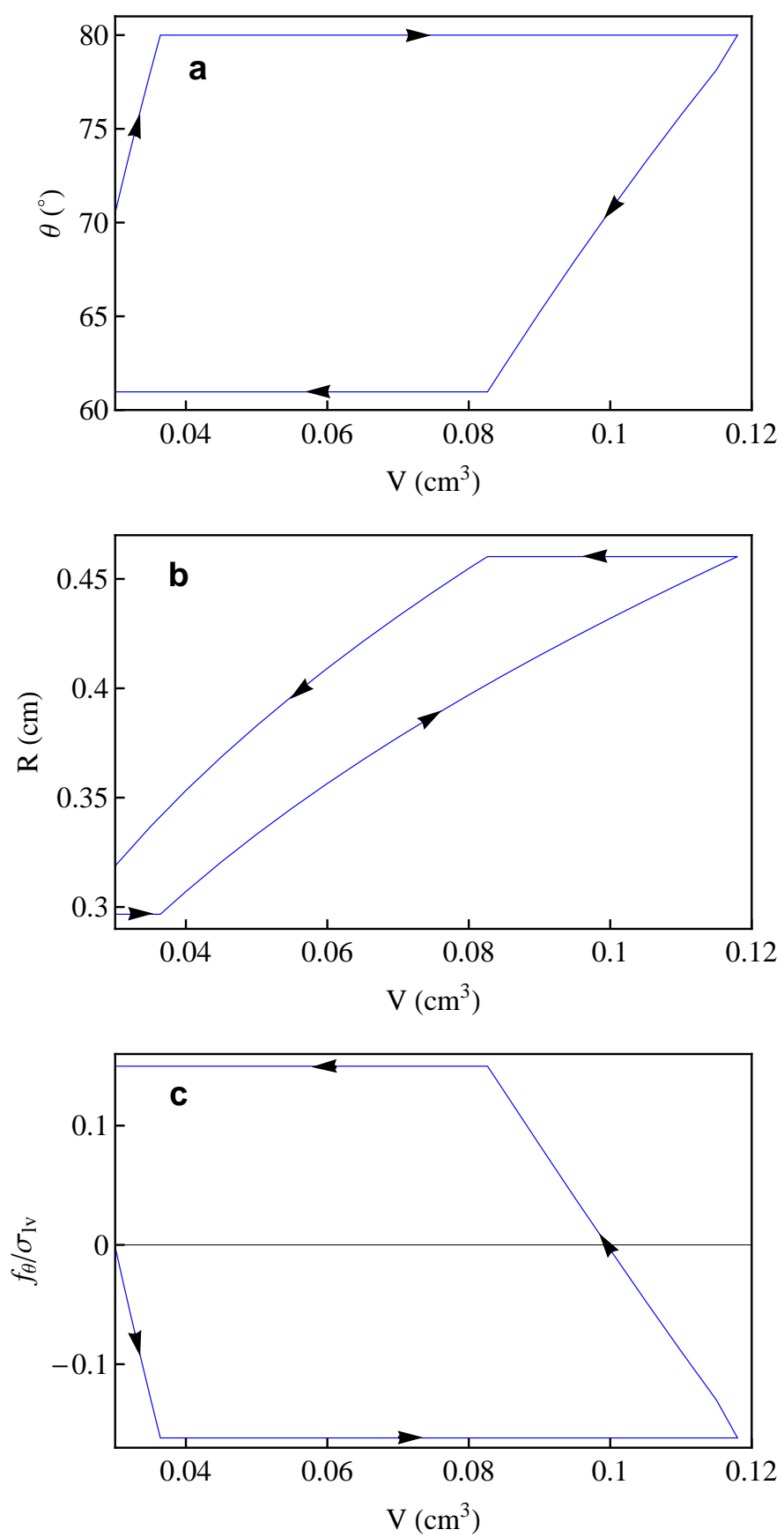

Figura 2.3: Ciclo de histéresis para una gota de agua sobre una superficie de silicio recubierta de ácido poliláctico. a) Ángulo de contacto $\theta$ frente al volumen $V$. b) Radio de la línea de contacto $R$ frente al volumen $V$. c) Fuerza de fricción adimensional $f_{\theta} / \sigma_{\mathrm{lv}}$ frente al volumen $V$. 
posición. En el capítulo siguiente se trabajará con gotas no-axisimétricas para las cuales la solución de la ecuación de Young-Laplace puede obtenerse recurriendo a un tratamiento de elementos finitos como el que aporta el software Surface Evolver [42]. Obviamente, el método basado en Surface Evolver también puede aplicarse a sistemas axisimétricos, como se verá a continuación.

\subsection{Implementación en Surface Evolver}

Existen trabajos previos que muestran cómo obtener histéresis de ángulo de contacto por medio de Surface Evolver, como es el caso de Brandon et al. [23] que presentan simulaciones para superficies planas químicamente heterogéneas que dan lugar a histéresis angular. Surface Evolver ha sido también utilizado para reproducir histéresis del ángulo de contacto en superficies microestructuradas [20, 25-28]. En todos estos casos el sistema tiene un funcional de energía bien definida y el proceso de minimización con unas ligaduras de Surface Evolver está, también, bien definido.

El modelo presentado en el apartado anterior para dar lugar a histéresis angular no puede ser implementado directamente en Surface Evolver porque considera una fuerza de fricción empírica que da lugar a disipación y la energía del sistema no se conserva, lo cual descarta una adecuada minimización de la energía. A continuación se propone un método para implementar una fuerza de fricción empírica en Surface Evolver añadiendo unas ligaduras locales a la evolución del sistema hacia una configuración estable.

Como ya se ha expuesto en el subapartado 1.8.2, la energía de una gota sésil en gravedad puede expresarse como (1.31):

$$
\widetilde{\varepsilon}=\iint_{\mid V} d \widetilde{A}-\iint_{\text {sl }} \cos \theta d \widetilde{A}+\iiint_{\mid} \frac{\widetilde{z}}{\widetilde{l}_{0}^{2}} d \widetilde{V},
$$

donde la tilde $(\sim)$ indica cantidades adimensionales, utilizando la tensión interfacial del líquido-vapor $\sigma_{\mathrm{Iv}}$ y el volumen $V_{\mathrm{g}}$ de la gota como factores normalizadores.

El análisis de la forma de la gota a partir de la expresión anterior se realiza por medio de un principio variacional en el cual se minimiza $\widetilde{\varepsilon}$ respecto de la forma de la gota, sometida a la ligadura de volumen constante $\widetilde{V}=1$. Se puede demostrar que 
la ecuación de Young-Laplace (2.3) coincide con la ecuación de Euler-Lagrange del principio variacional. Es decir, la solución de la ecuación en derivadas parciales a que da lugar la ecuación de Young-Laplace es la misma que la solución proporcionada por la minimización del funcional integral (1.31).

Como es bien conocido, el software Surface Evolver de Brakke [42] proporciona un medio apropiado para resolver el problema variacional mencionado ya que está diseñado especialmente para encontrar la superficie mínima sujeta a un conjunto de ligaduras, por el método de elementos finitos.

\subsubsection{Fuerza de fricción}

A continuación se presentan los pasos principales del algoritmo que va a permitir introducir una fuerza de fricción en Surface Evolver.

1. Al comienzo del bucle de iteración se almacenan las coordenadas de los vértices $i$ que pertenecen a la línea de contacto: $\mathbf{x}_{i, \text { old }}=\mathbf{x}_{i}$.

2. Se realiza un desplazamiento de la gota para obtener las nuevas coordenadas de los vértices de la línea de contacto $\mathbf{x}_{i}=\mathbf{x}_{i, n e w}$ y para calcular la fuerza por unidad de longitud adimensional $f_{i} / \sigma_{\mathrm{lv}}$ que actúa sobre cada uno de estos vértices. Esta fuerza se obtiene en Surface Evolver proyectando los desplazamientos de los vértices sobre la normal de la línea de contacto y dividiendo por la longitud media de las dos aristas contiguas a los vértices que pertenecen a la línea de contacto. El signo de las proyecciones indica si el vértice está en situación de avance o de retroceso.

3. Para cada vértice en avance $\left|f_{i}\right|$ se compara con la fuerza máxima de avance por unidad de longitud $f_{\mathrm{av}}$ dada por la ecuación (2.8). Si $\left|f_{i}\right| \leq f_{\mathrm{av}}$ el vértice $i$ se mantiene fijo $\left(\mathbf{x}_{i}=\mathbf{x}_{i, \text { old }}\right)$. En cambio, si $\left|f_{i}\right|>f_{\mathrm{av}}$ se permite que el vértice se mueva de acuerdo con la expresión siguiente

$$
\mathbf{x}_{i}=\mathbf{x}_{i, \text { old }}+\left(\mathbf{x}_{i, \text { new }}-\mathbf{x}_{i, \text { old }}\right) \frac{\left|f_{i}\right|-f_{\mathrm{av}}}{\left|f_{i}\right|}
$$


4. De modo análogo, para cada vértice en retroceso $\left|f_{i}\right|$ se compara con la fuerza máxima de retroceso por unidad de longitud $f_{\text {re }}(2.9)$. Si $\left|f_{i}\right| \leq f_{\text {re }}$ el vértice en posición $i$ se mantiene fijo $\left(\mathbf{x}_{i}=\mathbf{x}_{i, \text { old }}\right)$. En cambio, si $\left|f_{i}\right|>f_{\text {re }}$ se permite que el vértice se mueva de acuerdo con la expresión siguiente

$$
\mathbf{x}_{i}=\mathbf{x}_{i, \text { old }}+\left(\mathbf{x}_{i, \text { new }}-\mathbf{x}_{i, \text { old }}\right) \frac{\left|f_{i}\right|-f_{\mathrm{re}}}{\left|f_{i}\right|} \text {. }
$$

En definitiva las ecuaciones (2.14) y (2.15) muestran que el desplazamiento del vértice se escala con el cociente $\left(\left|f_{i}\right|-f_{\mathrm{av}}\right) /\left|f_{i}\right|$ si el vértice está en avance y con el cociente $\left(\left|f_{i}\right|-f_{\mathrm{re}}\right) /\left|f_{i}\right|$ si el vértice está en retroceso para tener en cuenta la fricción.

5. Los pasos anteriores se repiten hasta que se alcanza la convergencia.

La figura 2.4 muestra un diagrama de flujo del algoritmo utilizado para introducir un término de fricción en Surface Evolver. Este algoritmo debe usarse en lugar del comando usual g de Surface Evolver. Se denota como gfric al conjunto de comandos que implementa el algoritmo de la figura 2.4. Después de iniciarlo, solo se tiene que iterar este comando para obtener una forma estable de la gota para un volumen dado. Es de destacar que los únicos datos de entrada necesarios son los ángulos de contacto de avance y retroceso así como los necesarios para el ángulo de contacto de Young. En este trabajo se toma para $\theta_{Y}$ el valor medio de los ángulos de avance y retroceso, dado por la ecuación (2.10), aunque la expresión (2.11) basada en el valor medio de los cosenos da resultados muy similares.

\subsubsection{Medida del ángulo de contacto}

La manera habitual de medir los ángulos de contacto en Surface Evolver consiste en calcular directamente los ángulos entre el substrato y las caras triangulares que tienen una arista en la línea de contacto triple. Este planteamiento directo es muy fácil de implementar y arroja unos resultados relativamente buenos. Como se verá más adelante, estas medidas directas tienen el inconveniente de que se ven severamente afectadas por efectos del tamaño de la malla, por lo que hay que considerar un número grande de vértices en la triangulación de la gota para obtener buenos resultados. 


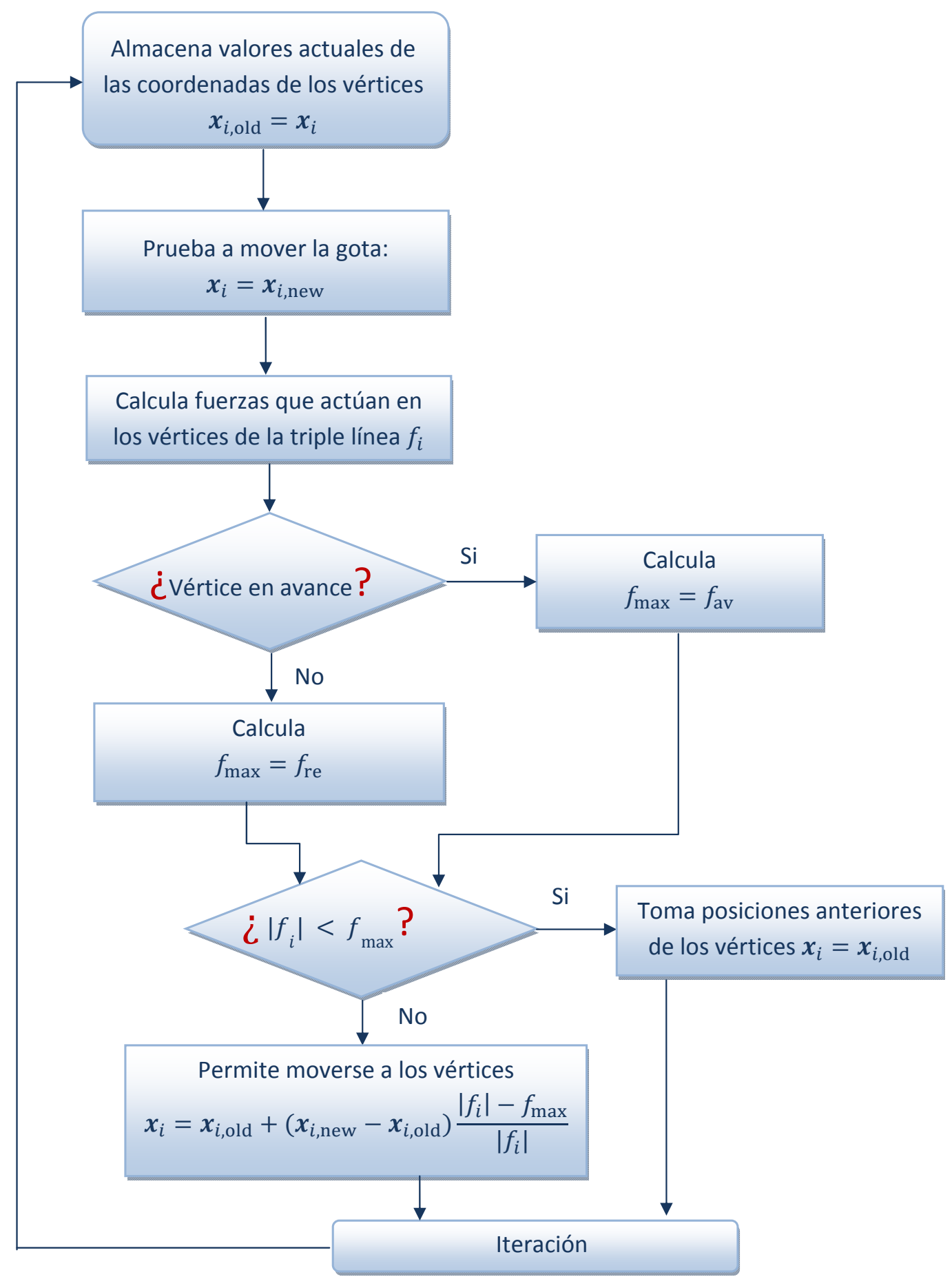

Figura 2.4: Diagrama de flujo del algoritmo utilizado en Surface Evolver. 
Ya que el algoritmo de la figura 2.4 está basado en la medida de las fuerzas $f_{i}$ que actúan sobre los vértices de la línea de contacto triple, a continuación se propone un método alternativo para las medidas de los ángulos de contacto en el que se hace uso de las relaciones entre las fuerzas y los ángulos de contacto que se han obtenido en el subapartado anterior. Más concretamente, a partir de la ecuación (2.13), para una situación de retroceso se tiene

$$
\theta_{i}= \begin{cases}\theta_{\text {re }} & \text { si }\left|f_{i}\right| \geq f_{\text {re }}, \\ \arccos \left(\cos \theta_{Y}+\left|f_{i}\right| / \sigma_{\mathrm{lv}}\right) & \text { si }\left|f_{i}\right|<f_{\text {re }},\end{cases}
$$

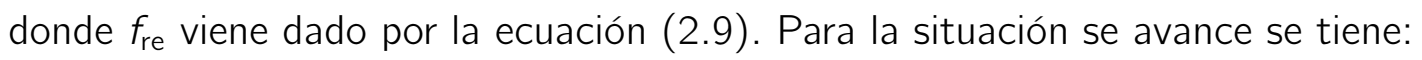

$$
\theta_{i}= \begin{cases}\theta_{\mathrm{av}} & \text { si }\left|f_{i}\right| \geq f_{\mathrm{av}}, \\ \arccos \left(\cos \theta_{\mathrm{Y}}-\left|f_{i}\right| / \sigma_{\mathrm{lv}}\right) & \text { si }\left|f_{i}\right|<f_{\mathrm{av}},\end{cases}
$$

donde $f_{\mathrm{av}}$ viene dado por la ecuación (2.8). En las ecuaciones (2.16) y (2.17) la notación $\theta_{i}$ indica que éste es el ángulo de contacto obtenido de la fuerza $F_{i}$ medida en el vértice $i$. La fuerza por unidad de longitud se obtiene de modo inmediato de

$$
f_{i}=\frac{2 F_{i}}{l_{i, 1}+l_{i, 2}}
$$

donde $l_{i, 1}$ y $l_{i, 2}$ son las longitudes de las dos aristas adyacentes al vértice $i$ que pertenecen a la línea triple. De hecho, $\left|F_{i}\right| / \sigma_{\mathrm{lv}}$ se puede medir directamente en Surface Evolver por medio de desplazamientos virtuales de la línea triple y, a partir de las ecuaciones (2.18) y (2.16) o (2.17) se obtiene el ángulo de contacto $\theta_{i}$. Para gotas axisimétricas es conveniente determinar el valor medio del ángulo de contacto a lo largo de la línea de contacto triple. En este trabajo se va a emplear la siguiente expresión para al ángulo de contacto medio $\bar{\theta}$ [23]

$$
\bar{\theta}=\frac{\oint_{C} \theta d l}{\oint_{C} d l},
$$

donde la integral de línea se ha establecido a lo largo del camino cerrado $C$ definido por la línea de contacto triple y $\theta$ es el ángulo de contacto local medido a lo largo de $C$. En el caso en estudio la ecuación (2.19) se reduce a

$$
\bar{\theta} \approx \frac{\sum_{i=1}^{N} \theta_{i}\left(l_{i, 1}+l_{i, 2}\right)}{\sum_{i=1}^{N}\left(l_{i, 1}+l_{i, 2}\right)},
$$




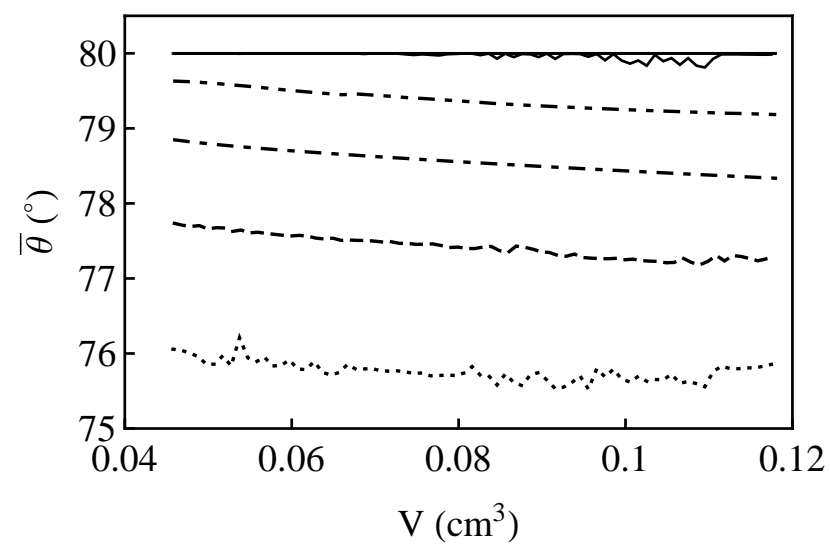

Figura 2.5: Valor medio del ángulo de contacto para una gota de agua sobre una superficie de silicio recubierta de ácido poliláctico frente al volumen en una situación de avance. Líneas continuas: resultado del nuevo método basado en la medida de las fuerzas. Otras líneas: resultado de la medida directa de los ángulos de contacto $N=24$ (línea de puntos), $N=48$ (línea discontinua), $N=96$ (línea punto-raya), y $N=192$ (línea punto-punto-raya).

donde $N$ es el número de vértices en la línea triple. Es de destacar que la longitud de la línea de contacto triple es simplemente

$$
L=\oint_{C} d l \approx \frac{1}{2} \sum_{i=1}^{N}\left(l_{i, 1}+l_{i, 2}\right) .
$$

Para comprobar la validez del nuevo método de medida de ángulos de contacto se ha considerado el mismo sistema de la figura 2.3 en una situación de avance en la cual el volumen de la gota aumenta de $V=0,046 \mathrm{~cm}^{3}$ a $V=0,118 \mathrm{~cm}^{3}$. En la figura 2.5 se presentan los resultados del valor medio del ángulo de contacto $\bar{\theta}$ medido tanto con el método directo como con el nuevo para diferentes valores del número de vértices de la línea triple, $N=24,48,96$ y 192 , que se corresponden con un número total de vértices $N_{v}=133,505,1967$ y 7777 , respectivamente. No se han considerado redes mayores debido al crecimiento exponencial del coste computacional. Como se puede observar el nuevo método arroja resultados muy buenos para valores de $N \geq 48$, observándose algo de ruido para $N=24$. El método de medida directa 


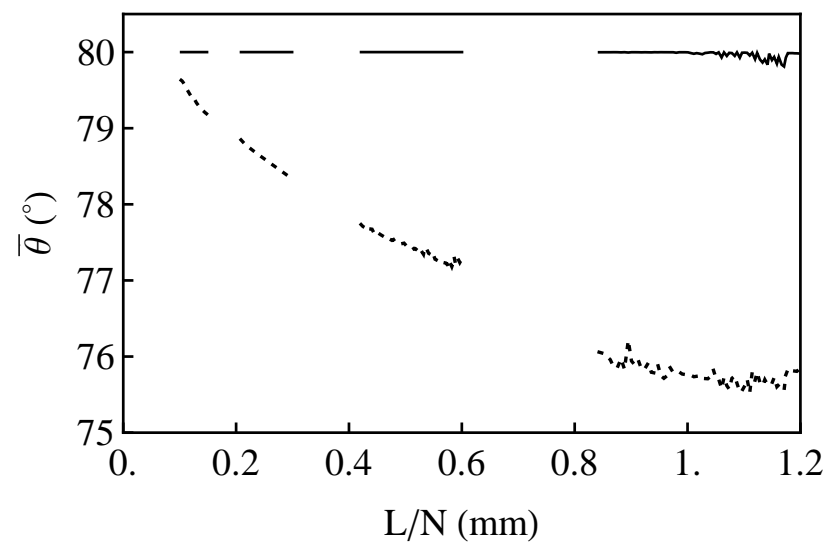

Figura 2.6: Valor medio del ángulo de contacto para una gota de agua sobre una superficie de silicio recubierta de ácido poliláctico frente al valor de la longitud principal de la línea triple $L / N$. Líneas continuas: resultado del nuevo método basado en la medida de las fuerzas. Líneas discontinuas: resultado de la medida directa de los ángulos de contacto.

está sujeto a efectos de tamaño de la malla en dos aspectos: por un lado, para un volumen dado arroja resultados que dependen fuertemente del número de vértices de la línea triple, con un error considerable incluso para $N=192\left(N_{v}=7777\right)$; por otro lado, para un número de vértices dado la medida del valor medio del ángulo de contacto depende del volumen por lo que decrece ligeramente cuando aumenta el volumen de la gota. Esto significa que el resultado del método de medida directa depende del espaciado de la malla, es decir, de la longitud media de las aristas en la teselación triangular de la gota. Esta dependencia se puede observar en la figura 2.6 donde se ven los resultados de la figura 2.5 como una función de la longitud media de las aristas de la línea triple $L / N$.

Para mostrar un análisis más detallado de los métodos de medida se ha considerado una gota de agua con un volumen fijo de $V=0,03 \mathrm{~cm}^{3}$ y un espaciado de malla tal que tiene $N=48$ vértices en la línea triple. En la simulación con Surface Evolver de este sistema particular se ha variado el ángulo de contacto de Young desde $\theta_{Y}=30^{\circ}$ a $150^{\circ}$, alcanzando el equilibrio para cada valor de $\theta_{Y}$, y entonces se 


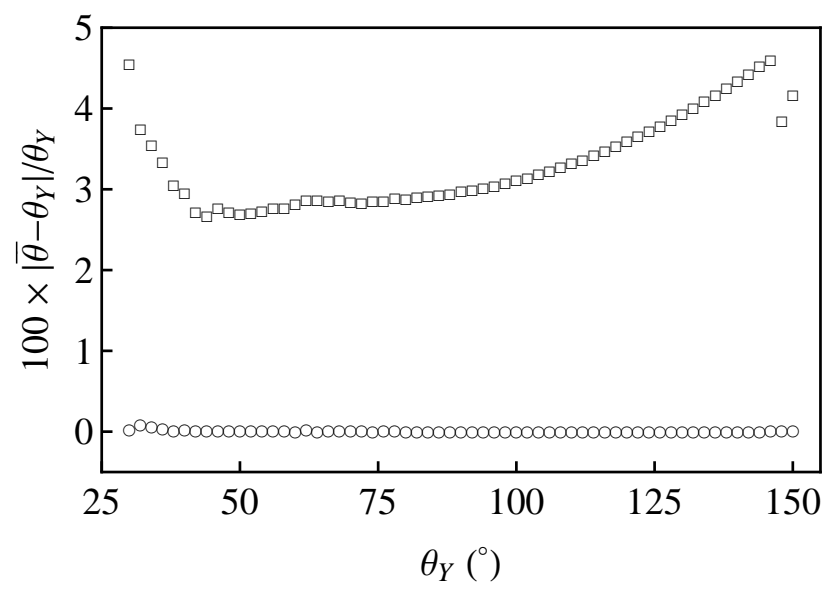

Figura 2.7: Porcentaje absoluto de las desviaciones relativas de las medidas del valor medio de los ángulos de contacto a partir del ángulo de contacto de Young para una gota de agua con $V=0,03$ $\mathrm{cm}^{3}$ y $\mathrm{N}=48$. Círculos: resultado del nuevo método basado en la medida de las fuerzas. Cuadrados: resultado de la medida directa de los ángulos de contacto.

ha medido el ángulo de contacto medio utilizando ambos métodos. En la figura 2.7 se representa el porcentaje absoluto de las desviaciones relativas de las medidas del valor medio de los ángulos de contacto a partir del ángulo de contacto de Young. Para los ángulos considerados se obtiene un valor medio de la desviación relativa absoluta de 3,3\% para el método de medida directa y de 0,0066\% para el nuevo método.

Teniendo en cuenta los buenos resultados del nuevo método de medida de ángulos de contacto, a partir de ahora se considerará únicamente este método.

\subsection{Comparación con resultados experimentales}

\subsubsection{Comparación con los resultados de Lam et al.}

En este subapartado se van a comparar los resultados de simulación con los datos experimentales de Lam et al. [43] para medidas DOCA de los ángulos de contacto 
de avance y retroceso para gotas sésiles. Considérense tres situaciones diferentes dependiendo del comportamiento del ángulo de retroceso:

- Ángulo de contacto de retroceso constante.

La figura 2.8 muestra los resultados para una gota de agua sobre láminas de silicio cubiertas de ácido poliláctico. Este caso, ya considerado en la figura 2.3, es un excelente ejemplo de ángulo de contacto de retroceso constante. Se comparan los resultados teóricos obtenidos a partir de la ecuación de Young-Laplace (línea discontinua) con la simulaciones de Surface Evolver (línea continua) y los datos experimentales obtenidos a partir de la figura 3 de la Ref. [43] (puntos). Los datos de entrada para los cálculos son $\theta_{\mathrm{av}}=79,82^{\circ}$ y $\theta_{\mathrm{re}}=60,97^{\circ}$. Además se ha utilizado el valor $g=9,8 \mathrm{~m} / \mathrm{s}^{2}$ de la aceleración de la gravedad, $\sigma_{\mathrm{lv}}=73 \mathrm{mN} / \mathrm{m}$ para la tensión superficial del agua, y $\rho=10^{3} \mathrm{~kg} / \mathrm{m}^{3}$ para la densidad del agua. El volumen máximo de la gota $\left(0,118 \mathrm{~cm}^{3}\right)$ y la velocidad de aumento y disminución del volumen se han obtenido de la figura 2.8c. En la figura 2.8a se muestra la variación del ángulo de contacto con el tiempo, observando un excelente acuerdo entre los resultados teóricos, la simulación y los datos experimentales. Hay que destacar que los datos experimentales comienzan cuando el sistema está ya en la situación de avance, mientras que los resultados teóricos y la simulación se han obtenido para un ciclo que comienza con el volumen inicial de $0,030 \mathrm{~cm}^{3}$ con un ángulo de contacto inicial igual al ángulo de contacto de Young que se supone toma el valor $\theta_{Y}=\left(\theta_{a d v}+\theta_{r e c}\right) / 2=70,40^{\circ}$. La variación con el tiempo del radio de la línea triple se muestra en la figura 2.8b donde teoría y simulación muestran un acuerdo excelente, con una ligera subestimación de los datos experimentales para ángulos de contacto de avance, y una ligera sobreestimación de los datos experimentales para ángulos de contacto de retroceso.

- No existe ángulo de contacto de retroceso

La figura 2.9 considera un sistema consistente en una gota de formamida sobre una superficie de silicio cubierta de ácido poliláctico-co-glicólico 50/50. En este caso no existe ángulo de contacto de retroceso. Los datos experimentales se 

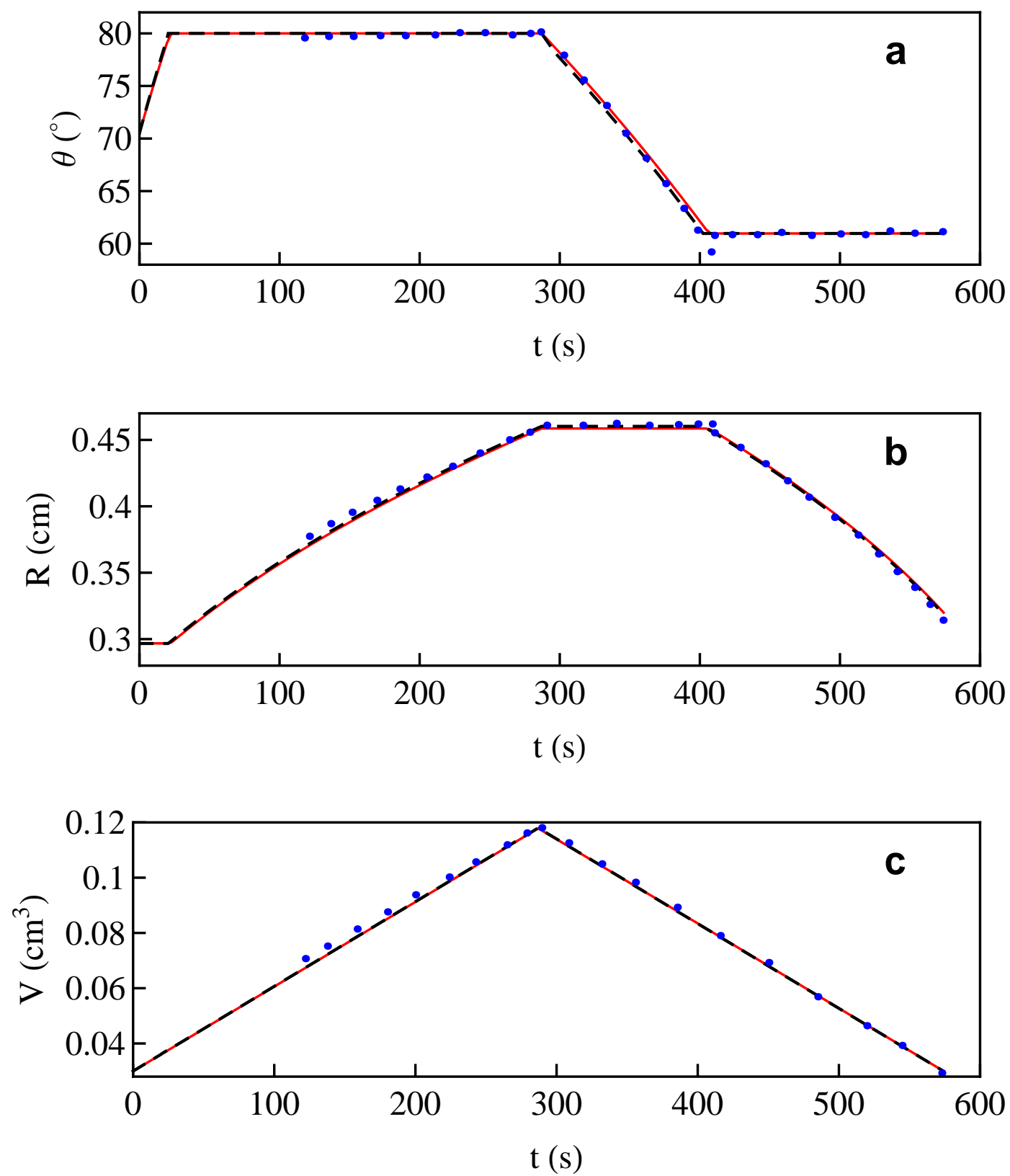

Figura 2.8: Histéresis del ángulo de contacto para una gota de agua sobre una superficie de silicio recubierta de ácido poliláctico. a) Ángulo de contacto $\theta$ frente al tiempo. b) Radio de la línea triple $R$ frente al tiempo. c) Volumen $V$ frente al tiempo. Línea continua: resultados de la simulación con Surface Evolver. Línea discontinua: resultados teóricos obtenidos resolviendo la ecuación de Young-Laplace. Puntos: datos experimentales [43]. 
han obtenido de la figura 5 de la Ref. [43]. Los datos de entrada para los cálculos son $\theta_{\mathrm{av}}=61,5^{\circ}$ y $\theta_{\mathrm{re}}=0^{\circ}$. Se ha utilizado el valor $\rho=1,13 \times 10^{3} \mathrm{~kg} / \mathrm{m}^{3}$ para la densidad de la formamida y se ha considerado $\sigma_{\mathrm{lv}}=52,5 \mathrm{mN} / \mathrm{m}$ para ser consistentes con los resultados de $\sigma_{\mathrm{lv}}$ mostrados en la figura 5 de la Ref. [43]. Los resultados teóricos y experimentales se han obtenido para un ciclo que comienza con un volumen inicial $V=0,015 \mathrm{~cm}^{3}$ y un ángulo de contacto inicial igual a $30,75^{\circ}$. Como en el caso anterior, el volumen máximo $0,0975 \mathrm{~cm}^{3}$ y su velocidad de variación se han obtenido de los datos experimentales de la figura 2.9c. En este caso, teoría y simulación presentan un acuerdo excelente tanto para los ángulos de contacto como para el radio de la línea triple pero la comparación con los datos experimentales arroja un ajuste ligeramente peor que en el caso de la figura 2.8, subestimando el radio de la línea triple para ángulos de contacto de retroceso.

- Ángulo de contacto de retroceso dependiente del tiempo.

La figura 2.10 presenta una situación con un ángulo de contacto de retroceso dependiente del tiempo obtenido para formamida sobre una superficie de silicio recubierta de poli(metil metacrilato/n-butil metacrilato $)^{1}$. Los datos experimentales se han obtenido a partir de la figura 2 de la Ref. [43]. Como ya se ha mencionado, la mayoría de los sistemas líquido-sólido presentan ángulos de contacto de retroceso dependientes del tiempo. Ya que un enfoque microscópico de la situación está fuera del alcance de este artículo, seguimos el enfoque de Lam et al. [43], considerando un ajuste por mínimos cuadrados a los ángulos de contacto de retroceso dependientes del tiempo (para $t \approx 390 \mathrm{~s}$ ). Se obtiene:

$$
\theta_{\mathrm{re}}(t)=117,325-0,25833 t+0,00022935 t^{2}
$$

Extrapolando al tiempo para el cual el volumen comienza a disminuir se obtiene el ángulo de contacto de retroceso inicial $\theta_{\text {re,ini }}=\theta_{\text {re }}(285) \approx 62,3^{\circ}$. Se espera que este ángulo de contacto inicial sea el ángulo de contacto antes de que

\footnotetext{
1Polimetilmetacrilato: dentro de los plásticos de ingeniería se puede encontrar como poli(metacrilato de metilo), poly(methyl methacrylate) en inglés, también conocido por sus siglas PMMA; análogamente el poli n-butil metacrilato se conoce de otro modo como PBMA.
} 

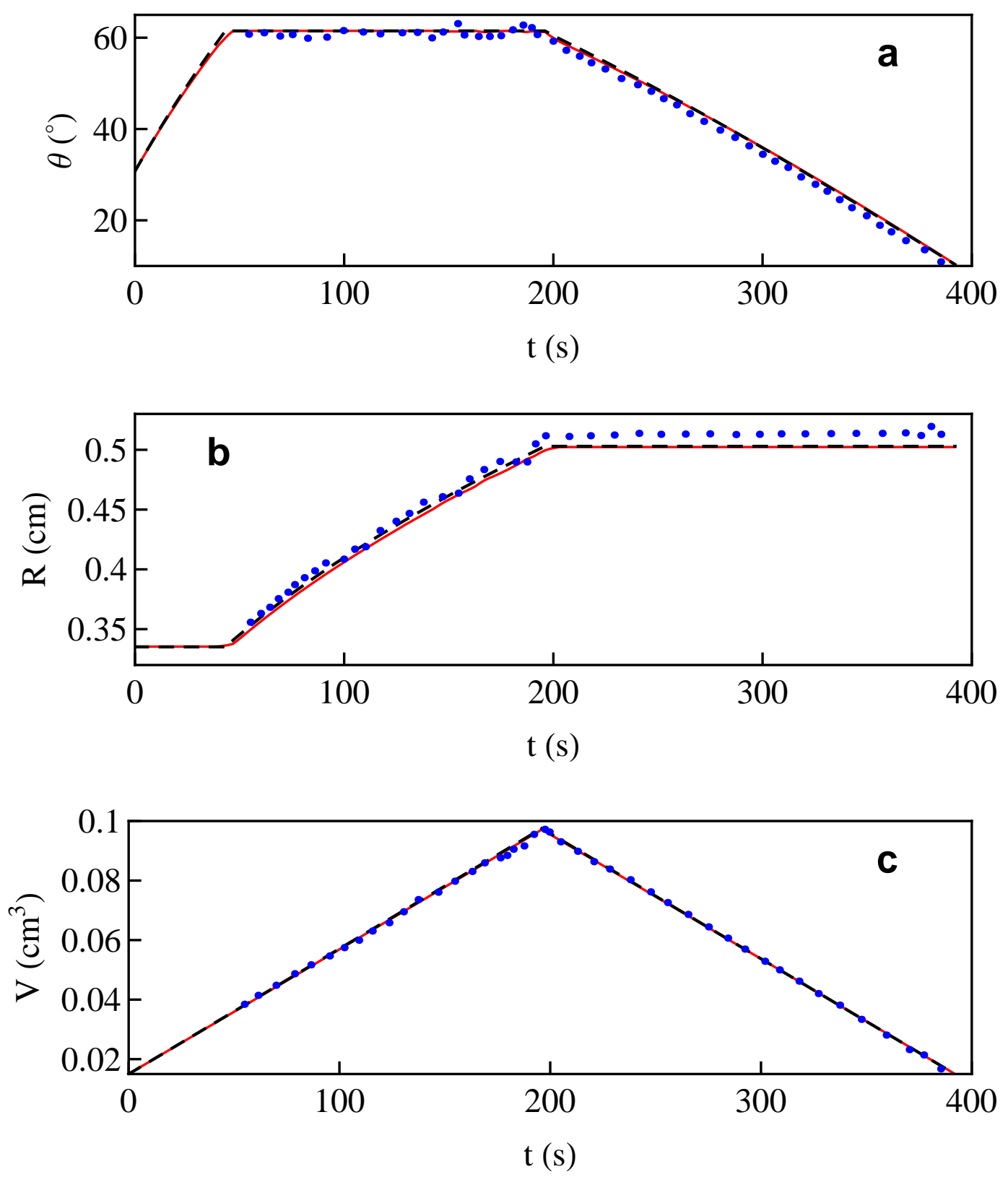

Figura 2.9: Ciclo de histéresis del ángulo de contacto para formamida sobre una superficie de silicio recubierta de ácido poliláctico-co-glicólico 50/50 a) Ángulo de contacto $\theta$ frente al tiempo. b) Radio de la línea triple $R$ frente al tiempo. c) Volumen $V$ frente al tiempo. Línea continua: resultados de las simulaciones con Surface Evolver. Línea discontinua: resultados teóricos obtenidos a partir de la ecuación de Young-Laplace. Puntos: datos experimentales [43]. 

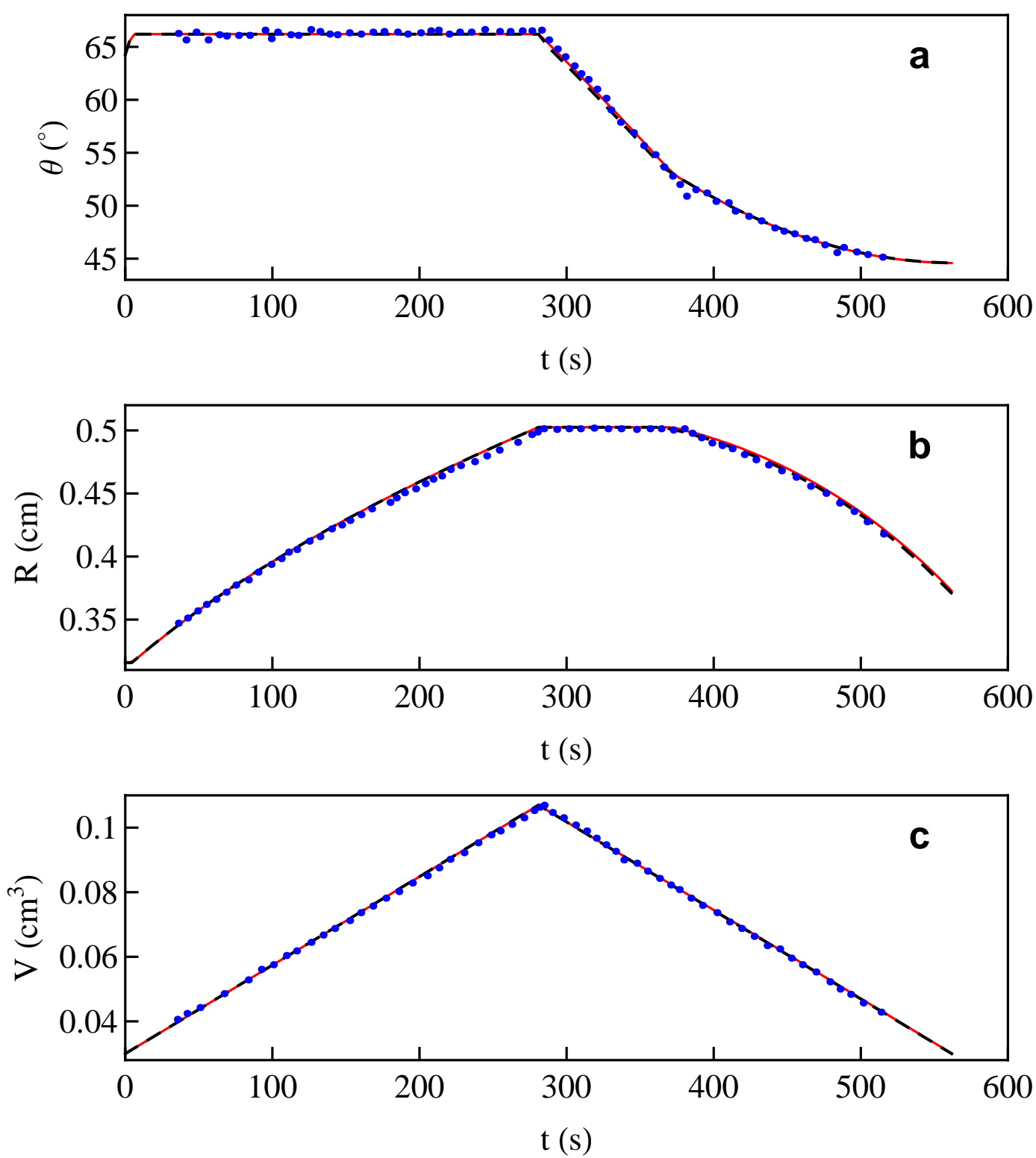

Figura 2.10: Ciclo de histéresis del ángulo de contacto para formamida sobre una superficie de silicio recubierta de poli(metil metacrilato/n-butil metacrilato). a) Ángulo de contacto $\theta$ frente al tiempo. b) Radio de la línea triple $R$ frente al tiempo. c) Volumen $V$ frente al tiempo. Línea continua: resultados de las simulaciones con Surface Evolver. Línea discontinua: resultados teóricos obtenidos a partir de la ecuación de Young-Laplace. Puntos: datos experimentales [43]. 
ninguna interacción sólido-líquido tenga lugar [43]. De los datos experimentales de la figura 2.10 se obtiene $\theta_{\mathrm{av}}=66,2^{\circ}$. Para asegurarnos la consistencia con los resultados de $\sigma_{\mathrm{lv}}$ de la figura 2 de la Ref. [43], en este caso particular se ha considerado el valor de $54,5 \mathrm{mN} / \mathrm{m}$ como tensión superficial de la formamida.

Los resultados teóricos y de simulación en la figura 2.10 han sido obtenidos para un ciclo que comienza con un volumen inicial de $0,03 \mathrm{~cm}^{3}$ y alcanza un valor máximo de $0,107 \mathrm{~cm}^{3}$. Se ha tomado como ángulo de contacto inicial $\left(\theta_{\text {re,ini }}+\right.$ $\left.\theta_{\mathrm{av}}\right) / 2=64,25^{\circ}$. Por supuesto, en lugar de utilizar un ángulo de retroceso constante, en este caso, cuando el volumen disminuye, se ha considerando un ángulo de retroceso dependiente del tiempo según la ecuación (2.22). Como en los casos anteriores, los resultados teóricos y de simulación presentan un ajuste excelente tanto para los ángulos de contacto como para el radio de la línea triple. La comparación con los datos experimentales también da un ajuste muy bueno para los ángulos de contacto mientras que para el radio de la línea de contacto triple está ligeramente sobreestimado.

\subsubsection{Comparación con los resultados de Vargha-Butler el al.}

Se ha utilizado el método expuesto anteriormente para reproducir los resultados experimentales de Vargha-Butler et al. [44] para una gota de agua sobre cinco superficies diferentes de ácido poliláctico, DL-PLA, y sus copolímeros con ácido poliglicólico DL-PLGA, en diferentes proporciones. Los datos utilizados para la simulación de un ciclo de histéresis con un volumen inicial $V_{\text {ini }}=0,03 \mathrm{~cm}^{3}$ en los cinco ejemplos estudiados se presentan a continuación:

a) Gota de agua sobre un superficie homopolímera de DL-PLA 100 :

$$
V_{\mathrm{fin}} \simeq 0,12 \mathrm{~cm}^{3} \quad \theta_{\mathrm{ini}}=70,42^{\circ} \quad \theta_{\mathrm{av}}=79,89^{\circ} \quad \theta_{\mathrm{re}}=60,95^{\circ}
$$

b) Gota de agua sobre una superficie copolímera DL-PLGA 85/15:

$$
V_{\text {fin }} \simeq 0,12 \mathrm{~cm}^{3} \quad \theta_{\text {ini }}=69,39^{\circ} \quad \theta_{\mathrm{av}}=79,07^{\circ} \quad \theta_{\mathrm{re}}=59,71^{\circ}
$$

c) Gota de agua sobre una superficie copolímera DL-PLGA 75/25:

$$
V_{\text {fin }} \simeq 0,23 \mathrm{~cm}^{3} \quad \theta_{\text {ini }}=68,4^{\circ} \quad \theta_{\mathrm{av}}=79,19^{\circ} \quad \theta_{\mathrm{re}}=57,64^{\circ}
$$



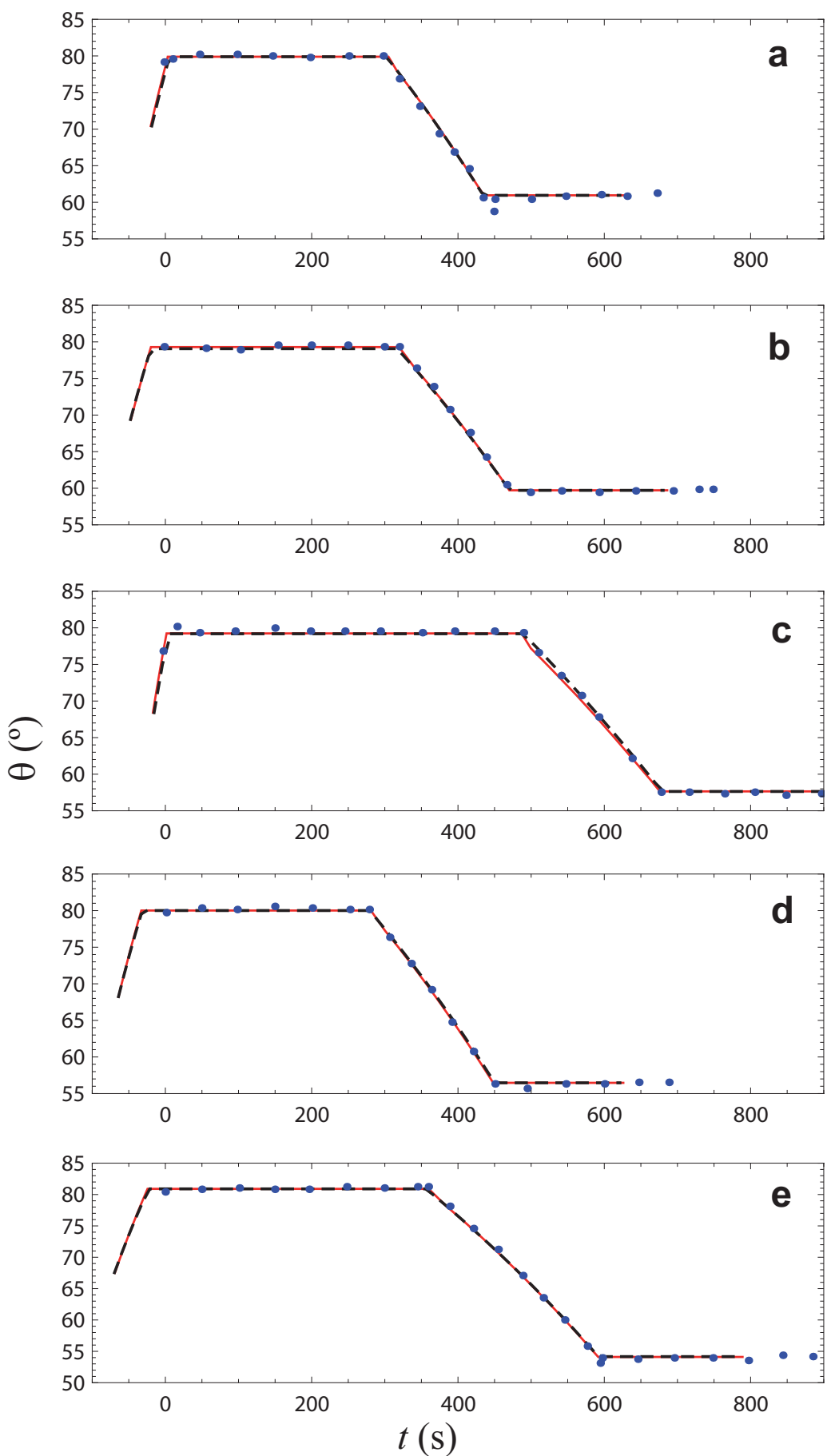

Figura 2.11: Evolución temporal del ángulo de contacto de una gota de agua en un ciclo de histéresis. Línea continua: resultados obtenidos resolviendo la ecuación de Young-Laplace en coordenadas cilíndricas. Línea discontinua: resultados de simulación con Surface Evolver. Puntos: datos experimentales de Vargha-Butlet el al. [44]. a) DL-PLA 100; b) DL-PLGA 85/15, c) DL-PLGA 75/25, d) DL-PLGA 65/35; e) DL-PLGA 50/50. 
d) Gota de agua sobre una superficie copolímera DL-PLGA 65/35:

$$
V_{\text {fin }} \simeq 0,12 \mathrm{~cm}^{3} \quad \theta_{\text {ini }}=68,24^{\circ} \quad \theta_{\mathrm{av}}=80,01^{\circ} \quad \theta_{\mathrm{re}}=56,47^{\circ}
$$

e) Gota de agua sobre una superficie copolímera DL-PLGA 50/50:

$$
V_{\text {fin }} \simeq 0,12 \mathrm{~cm}^{3} \quad \theta_{\text {ini }}=67,53^{\circ} \quad \theta_{\mathrm{av}}=80,90^{\circ} \quad \theta_{\mathrm{re}}=54,16^{\circ}
$$

Como en el caso anterior, es de destacar que los datos experimentales comienzan cuando el sistema está ya en la situación de avance, mientras que los resultados teóricos y los de simulación se han obtenido para un ciclo que comienza con un ángulo de contacto igual al ángulo de contacto de Young.

La comparación de los resultados experimentales con los de simulación se muestra en la figura 2.11, donde se representa la evolución temporal del ángulo de contacto de una gota de agua, en un ciclo de histéresis. Los resultados han sido obtenidos por medio de la ecuación de Young-Laplace en coordenadas cilíndricas (línea continua) y realizando simulaciones con Surface Evolver (línea discontinua). Los resultados experimentales de Vargha-Butlet el al. (puntos) [44] fueron obtenidos mediante una técnica ADSA-P. Es de destacar el excelente acuerdo entre los datos experimentales y los resultados teóricos y de simlación.

\subsection{Conclusiones}

En este capítulo se ha propuesto un modelo sencillo para el tratamiento de la histéresis de los ángulos de contacto de gotas sobre una superficie plana. A partir de los trabajos pioneros de Adam y Jessop [31] se ha incluido una fuerza de fricción por unidad de longitud en la ecuación de Young que permite establecer una relación entre ángulos de contacto y fuerzas de fricción y obtener las fuerzas máxima de avance y de retroceso. La fuerza de fricción actúa sobre la línea de contacto triple de modo que se mantiene fija para ángulos de contacto comprendidos en el intervalo $\theta_{\text {re }} \leq \theta \leq \theta_{a v}$

Se ha aplicado el modelo para gotas axisimétricas utilizando la solución de la ecuación de Young-Laplace en coordenadas cilíndricas, obteniendo un ajuste notable con los resultados experimentales de experiencias DOCA. El modelo ha sido también 
implementado en Surface Evolver donde puede utilizarse tanto para gotas axisimétricas como para no-axisimetricas. Además, debido a la relación entre los ángulos de contacto y las fuerzas de fricción, midiendo las fuerzas que actúan sobre la línea triple se proporciona un nuevo método para la medida de los ángulos de contacto en Surface Evolver más preciso que la medida directa de dichos ángulos de contacto.

Los resultados de Surface Evolver para gotas axisimétricas arrojan un ajuste excelente con los datos obtenidos de experimentos DOCA, incluso para situaciones con ángulos de contacto de retroceso dependientes del tiempo, aunque en este caso uno debe recurrir a una función de ajuste para los ángulos de contacto de retroceso, en lugar de utilizar un ángulo de retroceso constante como dato de entrada. 


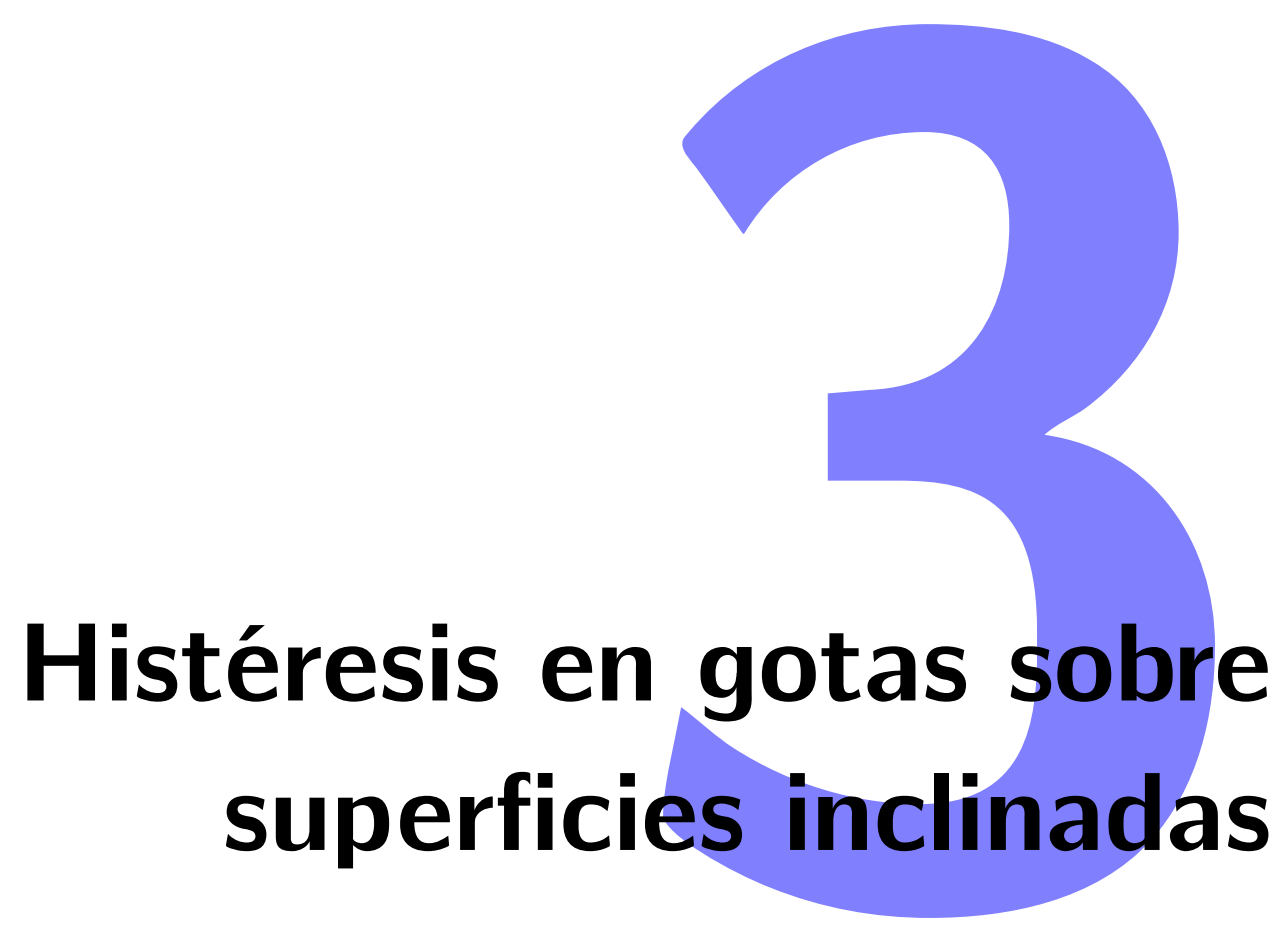

\subsection{Introducción}

La ecuación de Young-Laplace (1.16) determina que una gota sobre una superficie horizontal tiene una forma axisimétrica debido a la simetría cilíndrica del problema. Esta forma se convierte en no-axisimétrica cuando la superficie se inclina o gira a una velocidad angular dada. Para ángulos de inclinación $\alpha$ por debajo de un valor crítico $\alpha_{c}$ se observa que la gota se mantiene adherida a la superficie debido a un cambio de forma: la gota se deforma en la dirección de inclinación de manera que los ángulos de contacto en la parte delantera de la gota son mayores que los de la parte posterior (figura 3.1). La diferencia entre los ángulos de contacto de la gota se denomina histéresis del ángulo de contacto y ocasiona una fuerza de retención capilar que se compensa con la gravitatoria y evita el movimiento de la gota.

En la vida diaria pueden observarse frecuentemente gotas inclinadas que o bien se sujetan o se deslizan hacia abajo como en el caso de las gotas de lluvia sobre el parabrisas de un coche y en la hojas de las plantas.

El estudio de retención de gotas de líquido apoyadas sobre superficies inclinadas ha sido objeto de varios trabajos de investigación desde el artículo pionero de Mac- 


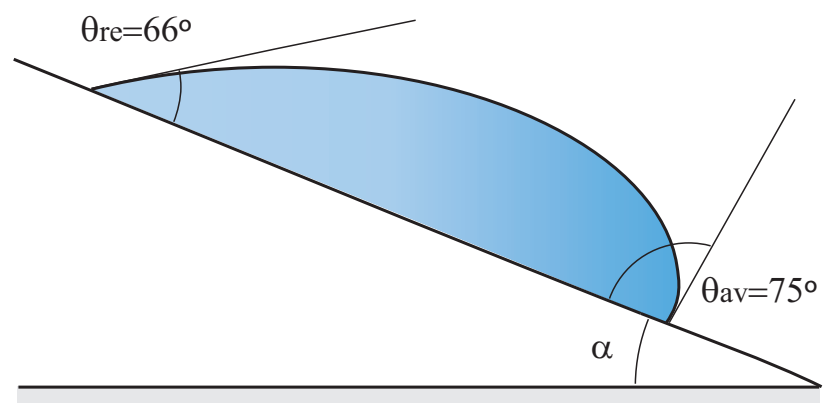

Figura 3.1: Sección de la gota sésil sobre un substrato sólido con un determinado ángulo de inclinación $\alpha$. Los ángulos de contacto en la parte delantera (ángulos de avance $\theta_{\mathrm{av}}$ ) de la gota son diferentes que los de la parte posterior (ángulos de retroceso $\theta_{\mathrm{re}}$ ).

Dougall y Ockrent en 1942 [48]. Estos autores, como una explicación fenomenológica de sus experimentos, determinaron que el producto del área $A$ de la sección transversal de la gota frente a sen $\alpha$ es proporcional a la diferencia entre los cosenos de los ángulos de contacto en la parte trasera o posterior (retroceso) $\cos \theta_{\text {post }}$, y la parte delantera o frontal (avance) $\cos \theta_{\text {front }}$, de la gota

$$
\rho g A \operatorname{sen} \alpha=\sigma_{\mathrm{lv}}\left(\cos \theta_{\text {post }}-\cos \theta_{\text {front }}\right) \text {, }
$$

donde $\rho$ es la densidad del líquido, $g$ es la aceleración debida a la gravedad, y $\sigma_{\mathrm{lv}}$ es la tensión superficial del líquido-vapor. Se debe destacar aquí que la ecuación (3.1) considera gotas para distintas inclinaciones, no críticas, y por lo tanto, en general, $\theta_{\text {post }}$ y $\theta_{\text {front }}$ son diferentes de los ángulos de contacto de retroceso y avance, $\theta_{\text {re }} \mathrm{y}$ $\theta_{\mathrm{av}}$, característicos de la inclinación crítica para la cual la gota comienza a deslizarse. MacDougall y Ockrent [48] también indicaron que la ecuación (3.1) se convierte en

$$
\rho g A \operatorname{sen} \alpha_{\mathrm{c}}=\sigma_{\mathrm{lv}}\left(\cos \theta_{\mathrm{re}}-\cos \theta_{\mathrm{av}}\right) \text {, }
$$

para el ángulo de inclinación crítico. Para un sistema dado esto implica que $A$ sen $\alpha_{c}$ es independiente del tamaño de la gota. Paralelamente, Frenkel obtuvo la misma 

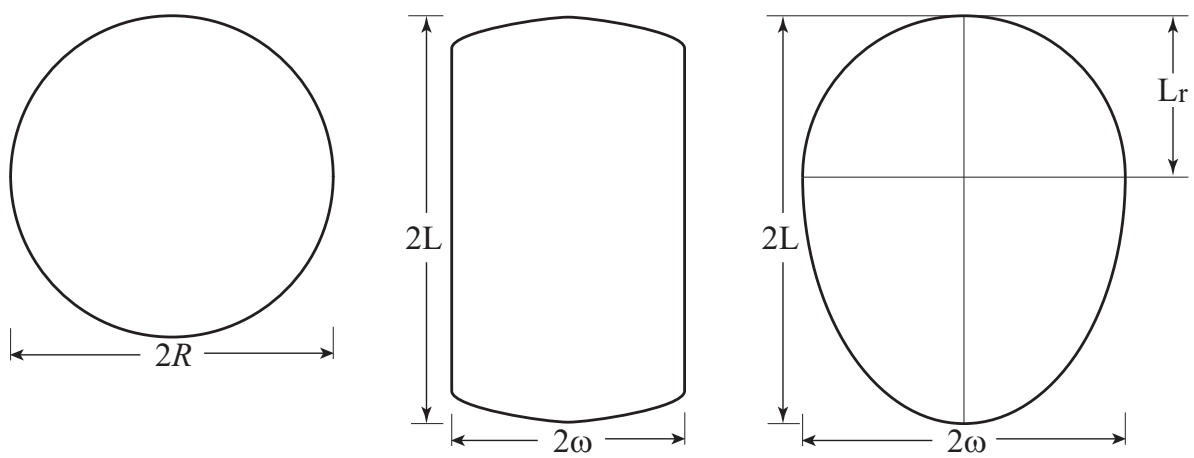

Figura 3.2: Aspecto de la base de la gota con diferentes formas de la línea de contacto triple: a) gota de base circular, b) gota de lados paralelos, y c) gota con asimetría de delante hacia atrás.

relación como una condición de contorno del problema de la estabilidad de una gota sobre una superficie inclinada [49]. Bikerman [50] señaló que, ya que el volumen de la gota es aproximadamente igual a $2 \omega A$, donde $2 \omega$ es la anchura de la gota en la dirección perpendicular a la inclinación, esto también implica que la relación $m g \operatorname{sen} \alpha_{c} / \omega=\rho g V \operatorname{sen} \alpha_{c}$ debería ser independiente del tamaño de la gota. Mas aún, la ecuación (3.2) sugiere que esta relación debe ser proporcional a la diferencia de $\operatorname{cosenos} \cos \theta_{\mathrm{re}}-\cos \theta_{\mathrm{av}}$. Este hecho fue observado por Furmidge [51] quien demostró que para gotas de lados paralelos a la inclinación crítica (figura 3.2b) se tiene

$$
\frac{\rho g \vee \operatorname{sen} \alpha_{\mathrm{c}}}{\sigma_{\mathrm{lv}}}=k \omega\left(\cos \theta_{\mathrm{re}}-\cos \theta_{\mathrm{av}}\right) \text {, }
$$

donde el factor de retención de la fuerza $k$ es igual a 2.

En la literatura se han propuesto diversos análisis y variaciones de la ecuación (3.3) utilizando argumentos teóricos, numéricos y experimentales y considerando diferentes formas para la línea de contacto. Larkin [52] comprobó la ecuación (3.3) frente a los resultados obtenidos a partir de cálculos numéricos de formas de la gota, encontrando desacuerdos que se atribuyeron al hecho de que los cálculos de Larkin se realizaron para gotas estáticas mientras que la ecuación (3.3) se aplica a gotas que se deslizan. Wolfram y Faust [32] consideraron gotas con línea de contacto triple circular (figura 3.2a) fija de radio $R$ (en este caso $\omega=R$ ) obteniendo $k=\pi$. Utilizando métodos de elementos finitos, Brown et al. [33] consideraron también una línea de 
contacto triple circular, pero ellos encontraron un valor de $k=\pi / 2$ para el factor de retención. Dussan y Chow [35,36] consideraron, como una aproximación para las gotas deslizándose, una línea de contacto triple formada por dos arcos semicirculares unidos por dos lados paralelos a lo largo de la dirección de inclinación (figura 3.2b). Suponiendo ángulos de contacto constantes para el arco de la parte frontal $\left(\theta_{\mathrm{av}}\right)$ y la parte posterior $\left(\theta_{\text {re }}\right)$ de la gota, obtuvieron el resultado de Furmidge [51], es decir, la ecuación (3.3) con $k=2$. Extrand y colaboradores $[53,54]$ Ilevaron a cabo estudios experimentales de gotas críticas encontrando que para baja histéresis la línea de contacto triple de la gota era aproximadamente circular, lo que dio lugar a una buena concordancia con la ecuación (3.3) con $\omega=R$ y $k=4 / \pi$ [53]. Para histéresis mayores, la línea de contacto triple de la gota se alargaba y se encontró que el valor de $k$ aumenta con el alargamiento y solamente se aproxima a $k=2$ para histéresis relativamente grandes [54]. Recientemente, EISherbini y Jacobi [55] propusieron una variación de la ecuación (3.3) para la cual $\omega$ se reemplazó por el radio equivalente $R_{\mathrm{a}}$ de un círculo con el mismo área que el de la base de la gota y se obtuvo, para el factor de retención de la fuerza $k$, un valor de $48 / \pi^{3}$. Experimentos muy recientes de Bouteau et al. [56] han encontrado que el comportamiento deslizante de gotas sobre superficies inclinadas de Langmuir-Blodgett se describe razonablemente bien con el modelo de Furmidge de la ecuación (3.3) con $k=2$. En la figura 3.3 se presenta una gráfica con los valores del factor de retención de la fuerza $k$ para la relación de aspecto $\beta=1$ (relación de aspecto de la gota $\beta=\frac{L}{\omega}$ ), obtenidos por los autores más relevantes que lo han estudiado a lo largo de la historia, considerando diferentes suposiciones tanto en el modo de realizar el estudio como en la forma de la base de la gota ${ }^{1}$.

En muchos casos las discrepancias anteriormente mencionadas se deben al uso de diferentes suposiciones teóricas o diferentes condiciones experimentales. Por ejemplo, los experimentos de Quéré et al. [57] y los de EISherbini y Jacobi $[55,58,59]$ fueron realizados para gotas depositadas sobre superficies previamente inclinadas mientras

\footnotetext{
${ }^{1}$ EISherbini and Jacobi [55] presentan una gráfica similar, pero en aquella se han comparado resultados de autores que no toman como referencia los mismos parámetros de la gota. En el gráfica (3.3) se toma como dato de la base de la gota en contacto con la superficie sólida su radio, o el radio equivalente $R_{\mathrm{a}}$, o bien el semiancho $\omega$
} 


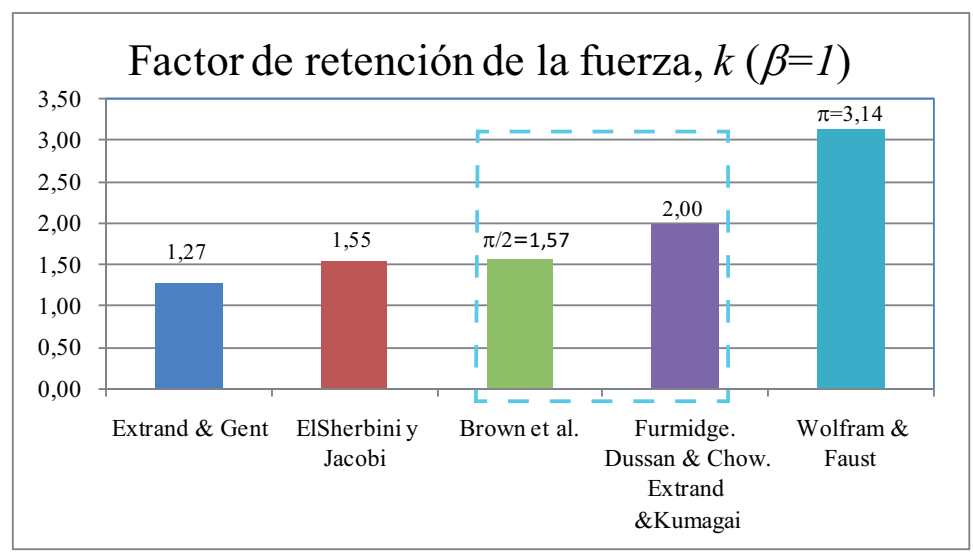

Figura 3.3: Valores del factor de retención de la fuerza $k$ para la relación de aspecto $\beta=1$, obtenidos por los autores que 10 han estudiado a lo largo de la historia, considerando diferentes suposiciones tanto en el modo de abordar el estudio como en la forma de la base de la gota. Los resultados de este trabajo (línea discontinua azul) se encuentran en el intervalo $\pi / 2<k<2$.

que los de Extrand y colaboradores o bien consideran una gota sobre una superficie horizontal que se inclina posteriormente [54] o una gota sobre una placa giratoria [53]. Pierce et al. [60] estudiaron diferentes métodos para realizar el experimento de placa inclinada, obteniendo desviaciones significativas en el ángulo crítico dependiendo del método. Otras fuentes de discrepancias pueden deberse al tiempo que la gota está depositada sobre la superficie antes del deslizamiento [61] o al ángulo de contacto inicial que la gota adopta como resultado de su colocación sobre la superficie [60, 62]. Desde un punto de vista teórico algunos investigadores consideran una línea de contacto circular [33] mientras que otros consideran gotas deslizantes de lados paralelos $[35,36]$. Resultados numéricos recientes han considerado simulaciones en las cuales se permite que se mueva la línea de contacto triple utilizando métodos de elementos finitos [37,38] o un enfoque de "problema de optimización" [39]. En 2005, Krasovitski y Marmur [5] consideraron un estudio teórico de gotas en dos dimensiones en el cual demostraron que los ángulos de contacto máximo y mínimo para gotas, sobre planos inclinados, no siempre coinciden con los ángulos de contacto de avance 
y retroceso. Se presenta en el cuadro 3.1 un resumen de lo expuesto anteriormente, en el que los valores resaltados en azul toman como dato de la base de la gota en contacto con la superficie sólida su radio, el radio equivalente, o el semiancho; esto hay que tenerlo en cuenta ya que algunos autores toman como referencia el ancho total de la gota y por lo tanto el valor de $k$ sería la mitad que para el caso de considerar el semiancho.

El objetivo del presente capítulo es analizar el problema considerando simulaciones de gotas sobre superficies inclinadas, mediante el método basado en Surface Evolver [42] presentado en el capítulo anterior [64]. El método es válido para gotas no-axisimétricas y por lo tanto puede ser aplicado al problema de las gotas sobre superficies inclinadas. Por supuesto, utilizando este método uno puede realizar medidas tanto para gotas críticas como no críticas. Esto permitirá analizar la evolución hacia el ángulo de inclinación crítico para diferentes condiciones iniciales de la simulación.

Este capítulo está estructurado como sigue. Se comienza con un resumen del método de simulación para gotas sobre superficies inclinadas. Posteriormente se considera la evolución de una gota de volumen constante sobre una superficie horizontal que se inclina lentamente para dos situaciones iniciales diferentes: (i) ángulo de contacto inicial igual a $\theta_{\mathrm{av}}$, y (ii) ángulo de contacto inicial igual a $\left(\theta_{\mathrm{av}}+\theta_{\mathrm{re}}\right) / 2$. A continuación se compara con los datos experimentales para una aleación líquida de $\mathrm{Sn}$-Ag-Cu sobre un substrato de cobre inclinado. Se realiza también un estudio de la deformación de la base de la gota para el caso $\theta_{\text {ini }}=\theta_{\mathrm{av}}$.

\subsection{Método de simulación}

Ya se ha visto que la forma de una gota sésil sometida a la aceleración debida a la gravedad $\mathbf{g}$ con un ángulo de contacto de Young $\theta_{Y}$, se puede obtener minimizando el funcional de energía [64]

$$
U=\sigma_{\mathrm{lv}} A_{\mathrm{lv}}-\iint_{A_{\mathrm{ls}}} \sigma_{\mathrm{lv}} \cos \theta_{Y} \mathrm{~d} A-\iiint_{V} \Delta \rho \mathbf{g} \cdot \mathbf{r} \mathrm{d} V,
$$

sujeto a la ligadura de volumen constante. En la ecuación (3.4) se ha mantenido de manera explícita el carácter vectorial de $\mathbf{g}$ y $\mathbf{r}$. Como siempre $A_{\mathrm{ls}}$ es el área del 


\begin{tabular}{|c|c|c|c|c|c|c|c|c|c|}
\hline 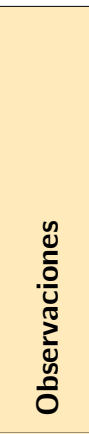 & 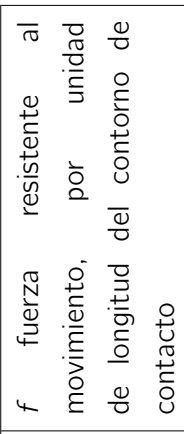 & $\begin{array}{l}N \\
\| \\
x \\
\Uparrow \\
\frac{x}{2} \\
11 \\
3 \\
i n\end{array}$ & $\begin{array}{l}0 \\
\| 1 \\
3 \\
\end{array}$ & & 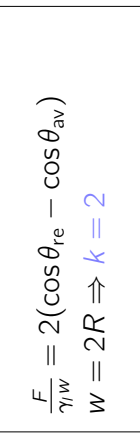 & & 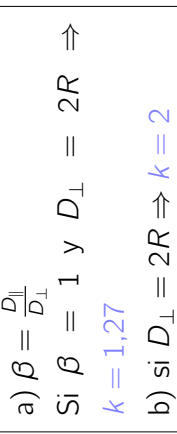 & 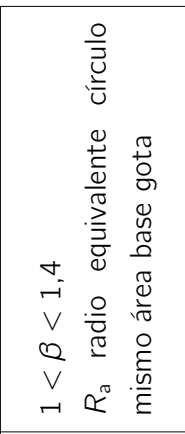 & \\
\hline 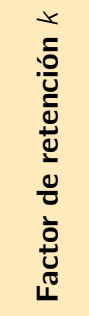 & 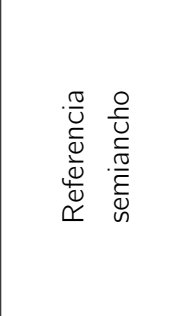 & 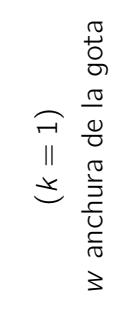 & $\begin{array}{l}k \\
\| \\
\forall\end{array}$ & 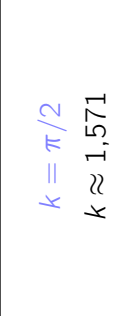 & 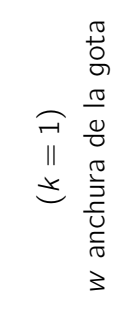 & 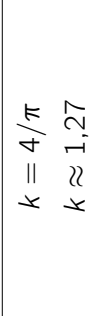 & 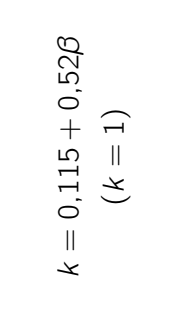 & 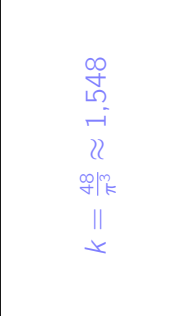 & \begin{tabular}{l}
$N$ \\
$\|$ \\
\multirow{*}{*}{}
\end{tabular} \\
\hline 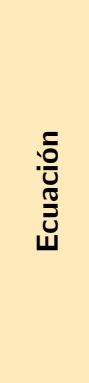 & 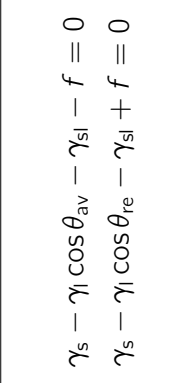 & 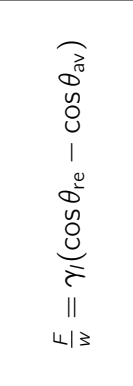 & 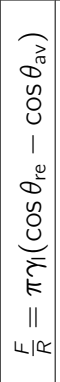 & 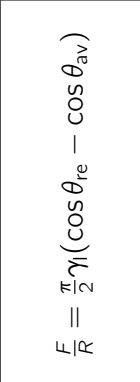 & 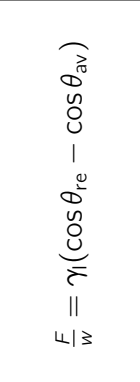 & 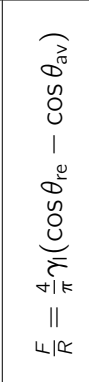 & 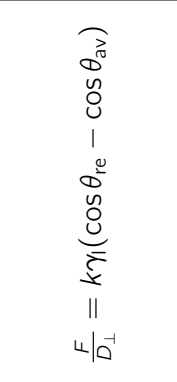 & 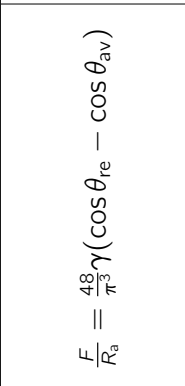 & 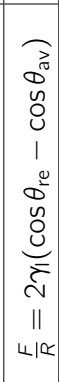 \\
\hline 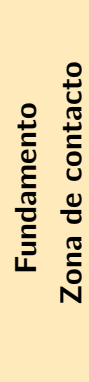 & 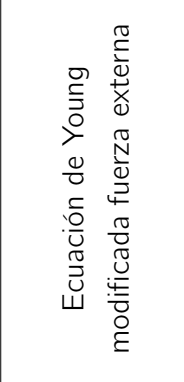 & 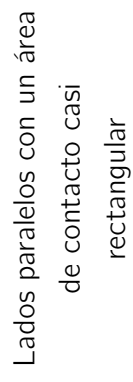 & 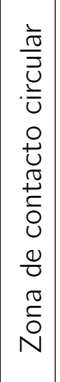 & 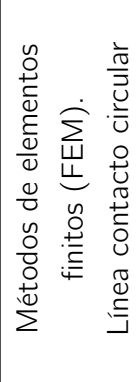 & 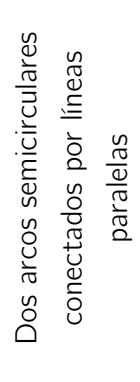 & 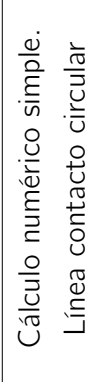 & 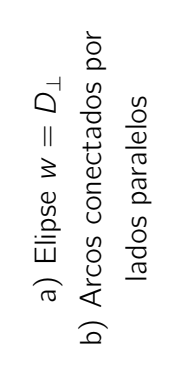 & 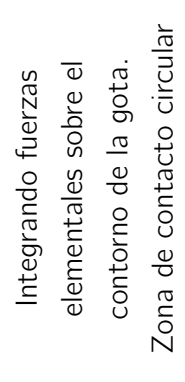 & 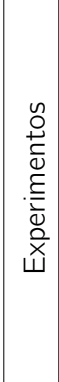 \\
\hline $\begin{array}{l}\text { y } \\
\stackrel{0}{0} \\
\stackrel{\Xi}{*}\end{array}$ & 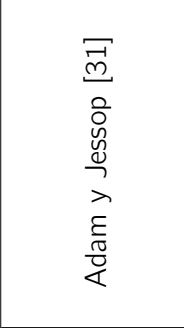 & 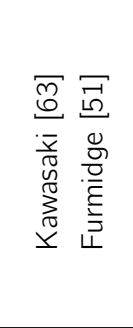 & 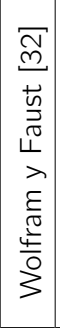 & 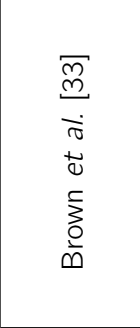 & 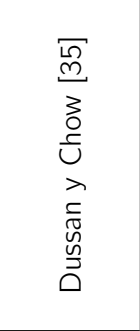 & 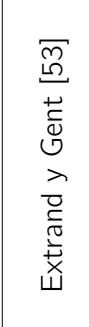 & 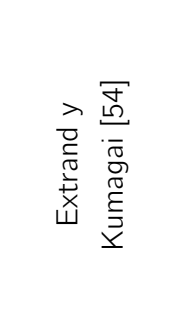 & 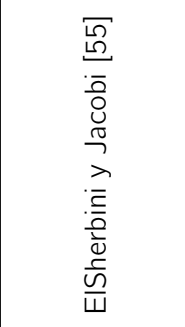 & 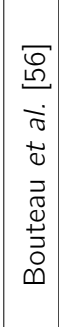 \\
\hline 突 & $\stackrel{\stackrel{\sim}{N}}{\underset{\sim}{\sigma}}$ & $\begin{array}{l}\stackrel{\circ}{\circ} \\
\stackrel{్}{\sigma}\end{array}$ & $\begin{array}{l}\infty \\
\stackrel{\sigma}{-} \\
-1\end{array}$ & $\begin{array}{l}\stackrel{\infty}{\circ} \\
\stackrel{\sim}{\rightarrow}\end{array}$ & $\begin{array}{l}\infty \\
\stackrel{\infty}{\sigma} \\
\stackrel{\neg}{न}\end{array}$ & ㅇ. & $\stackrel{\llcorner}{\stackrel{2}{\circ}}$ & ષัे & $\begin{array}{l}\infty \\
\stackrel{\sim}{े}\end{array}$ \\
\hline
\end{tabular}




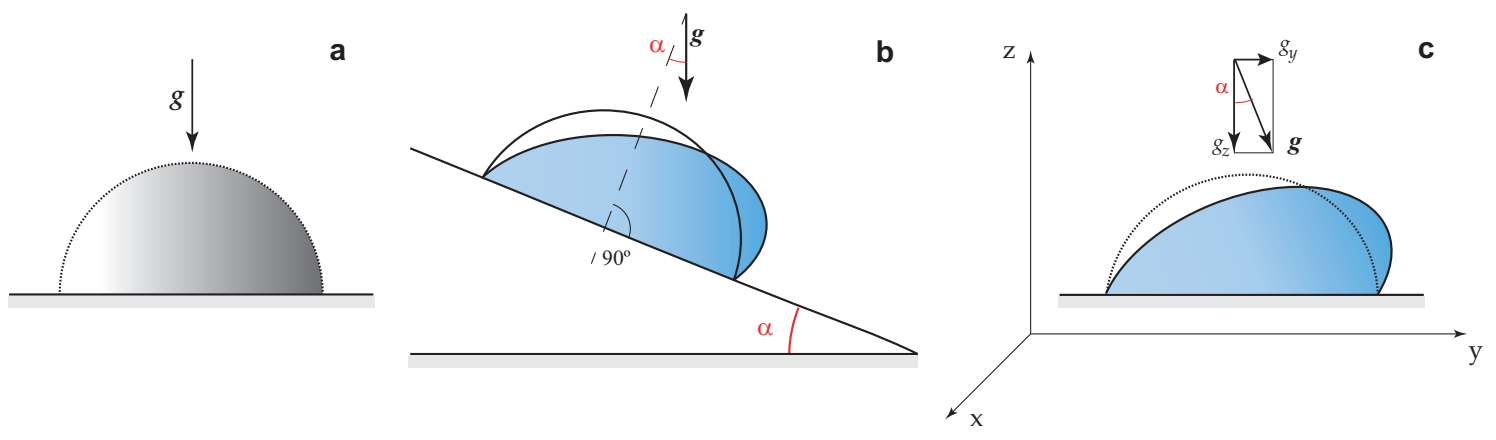

Figura 3.4: Planteamiento del problema para realizar el estudio de la inclinación del substrato en el que se encuentra depositada la gota sésil. a) Gota depositada en una superficie horizontal. b) Gota sobre un plano inclinado un ángulo a respecto a la horizontal. c) Gota sobre una superficie sin inclinación y con una componente horizontal de la gravedad $g_{y}$.

líquido en contacto con la superficie (el área de la base de la gota), $A_{\mathbb{l}}$ es el área de la interfaz líquido-vapor, y $\Delta \rho$ representa la diferencia de densidades entre las fases líquida y vapor $(\Delta \rho \approx \rho$, la densidad del líquido).

Como ya se ha comentado anteriormente el software Surface Evolver de Brakke [42] aporta un método de elementos finitos para resolver el problema variacional de la ecuación (3.4). Este software ha sido especialmente diseñado para encontrar la superficie mínima (de la gota) sujeta a una serie de ligaduras. La superficie de la gota se representa en Surface Evolver mediante una teselación con caras triangulares unidas por aristas que terminan en vértices.

En la figura 3.4 se muestra esquemáticamente la estrategia que se ha implementado en la simulación para abordar el estudio de gotas inclinadas sobre una superficie:

a) Si la gota está depositada en una superficie horizontal la acción de la aceleración debida a la gravedad es perpendicular a dicha superficie (figura 3.4a).

b) Cuando el plano en el que es encuentra depositada la gota se inclina un ángulo $\alpha$, el vector $\mathbf{g}$ sigue siendo vertical, pero presenta esa misma inclinación $\alpha$ respecto a la perpendicular al substrato en el que está depositada la gota, y su 
acción la deforma como se puede ver en la de la figura 3.4b.

c) Se puede obtener el mismo resultado que en el subapartado anterior, es decir gota sobre un plano inclinado un ángulo $\alpha$ respecto a la horizontal, seleccionando los ejes de coordenadas como se muestra en la figura 3.4c. Es decir, manteniendo la superficie sólida en el plano $x y$ e introduciendo una componente de la aceleración de la gravedad en la dirección del eje $y, g_{y}=g \operatorname{sen} \alpha$. De modo que el vector gravedad $\mathbf{g}$ quedaría definido por las componentes: $\mathbf{g}=\left(0, g_{y}, g_{z}\right)=(0, g \operatorname{sen} \alpha, g \cos \alpha)$. El aumento del ángulo de inclinación del plano se corresponde con el incremento de la fuerza externa $g_{y}=g \operatorname{sen} \alpha$ actuando sobre la gota.

Por lo tanto, un método directo de implementación de un ángulo de inclinación en Surface Evolver es considerar un vector gravedad "inclinado" $\mathbf{g}=(0, g \operatorname{sen} \alpha, g \cos \alpha)$ por lo que para $\alpha=0$ se recupera una aceleración debida a la gravedad $\mathbf{g}$ en la dirección del eje $z$ (vertical). Para $\alpha \neq 0$ se obtiene una contribución no nula en la dirección del eje y (la dirección de inclinación). La minimización de la ecuación (3.4) con Surface Evolver para una superficie inclinada $(\alpha \neq 0)$ daría lugar a una gota moviéndose en la dirección del eje y con aceleración $g$ sen $\alpha$ debido a que el funcional de la ecuación (3.4) no incluye un término de retención capilar basado en la histéresis del ángulo de contacto que podría equilibrar la gravedad en la dirección de inclinación. Sin embargo, el algoritmo que se ha desarrollado [64] para incluir la histéresis de ángulos de contacto en Surface Evolver, expuesto en detalle en el capítulo anterior y recogido en la figura 2.4 , permite minimizar adecuadamente la ecuación (3.4) para una superficie inclinada, añadiendo ligaduras locales al problema variacional.

Como resultado del mismo se puede calcular la forma de la gota sobre una superficie inclinada, la fuerza de retención capilar que actúa sobre cada vértice de la línea de contacto triple, así como medir con precisión los ángulos de contacto mediante las ecuaciones (2.16) y (2.17) [65].

En la figura 3.5 se muestra la forma de una gota durante el proceso de inclinación de la misma en la simulación con Surface Evolver. Se puede observar que los ángulos 


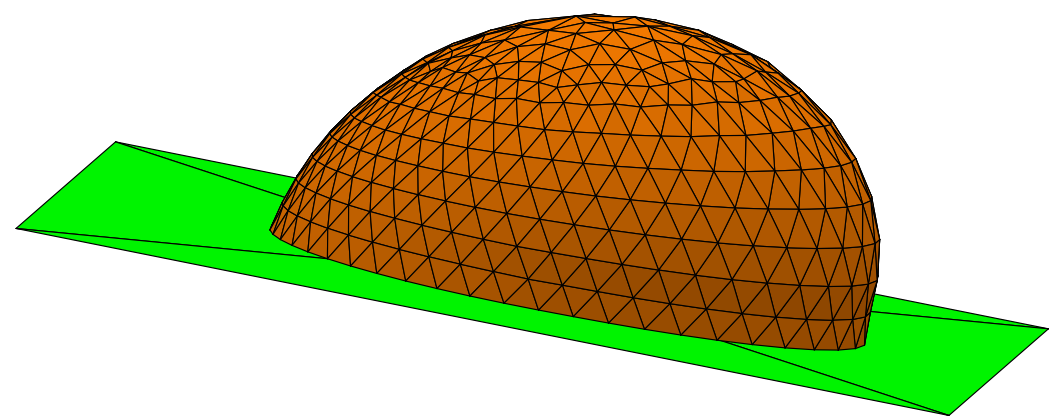

Figura 3.5: Forma de la gota durante el proceso de inclinación de la misma con el programa en Surface Evolver.

de contacto del frente de la gota son diferentes de los de la parte de atrás, es decir, la silueta es más achatada a la izquierda (se corresponde con la parte mas elevada del plano en la simulación) que a la derecha de la misma.

\subsection{Simulación de un proceso de inclinación}

En este apartado se simula el proceso según el cual un plano inicialmente horizontal se inclina lentamente hasta alcanzar el ángulo crítico para una gota en él depositada. Para llevar a cabo tal simulación con el algoritmo presentado se precisan únicamente unos pocos parámetros para describir la forma de la gota, las fuerzas de retención y los ángulos de contacto. Para una gota de líquido sobre una superficie dada estos parámetros de entrada son: el volumen de la gota $V$, la tensión superficial $\sigma_{\mathrm{lv}}$, la densidad del líquido $\rho$ y los ángulos de avance y retroceso para el sistema. Es conveniente recalcar aquí que, de acuerdo con los resultados de Krasovitski y Marmur [5], los ángulos de contacto de avance y retroceso para gotas sobre superficies inclinadas no siempre coinciden con los ángulos de contacto de avance y retroceso usuales. Adicionalmente uno puede establecer las condiciones iniciales para el sistema. Se han considerado dos situaciones iniciales diferentes: (i) ángulo de contacto inicial igual a $\theta_{\mathrm{av}}$, y $(\mathrm{ii})$ ángulo de contacto inicial igual a $\theta_{\mathrm{Y}}=\left(\theta_{\mathrm{re}}+\theta_{\mathrm{av}}\right) / 2$. 


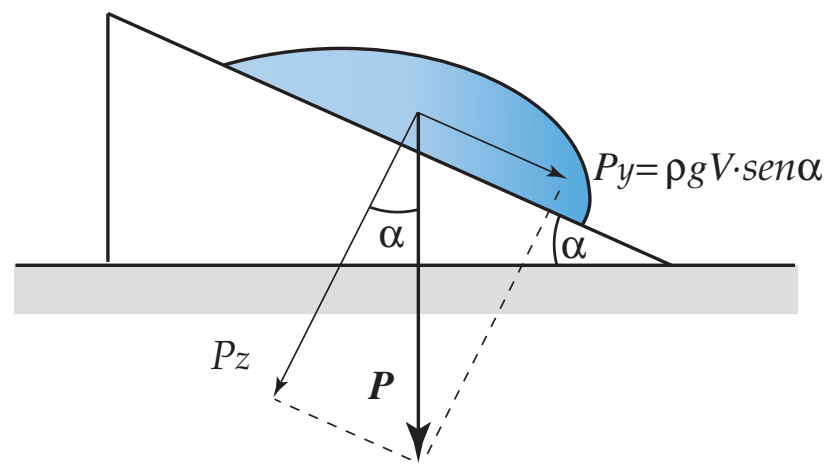

Figura 3.6: Componentes del peso al que se ve sometido una gota sobre un plano inclinado, según se ha seleccionado el sistema de ejes coordenados.

\subsection{1. Ángulo de contacto inicial igual a $\theta_{\mathrm{av}}$}

Se considera un ángulo de contacto inicial igual a $\theta_{\mathrm{av}}$ para una gota situada sobre una superficie horizontal que posteriormente se inclina, ya que esta situación se asemeja a las condiciones experimentales habituales [60]. Esta suposición ha sido considerada también en un trabajo muy reciente [29]. Se expone en primer lugar un estudio detallado de las fuerzas de retención y en segundo lugar la forma que adquiere la línea de contacto triple en función del ángulo de contacto.

\section{Fuerzas de retención}

En primer lugar, para comprobar el procedimiento de simulación se comienza con un análisis de las fuerzas de retención en la línea de contacto triple. Como la fuerza de retención que actúa sobre cada vértice de la línea de contacto triple puede medirse en la simulación con Surface Evolver, es fácil obtener la fuerza de retención total a lo largo de la dirección de inclinación $F_{y}$. Para una gota no crítica esta fuerza de retención total debe compensar la contribución gravitatoria $\rho g V \operatorname{sen} \alpha$ (figura 3.6). Este hecho proporciona una prueba de la convergencia de la simulación, ya que una situación de equilibrio se alcanza únicamente cuando estos dos términos de cancelan mutuamente. 

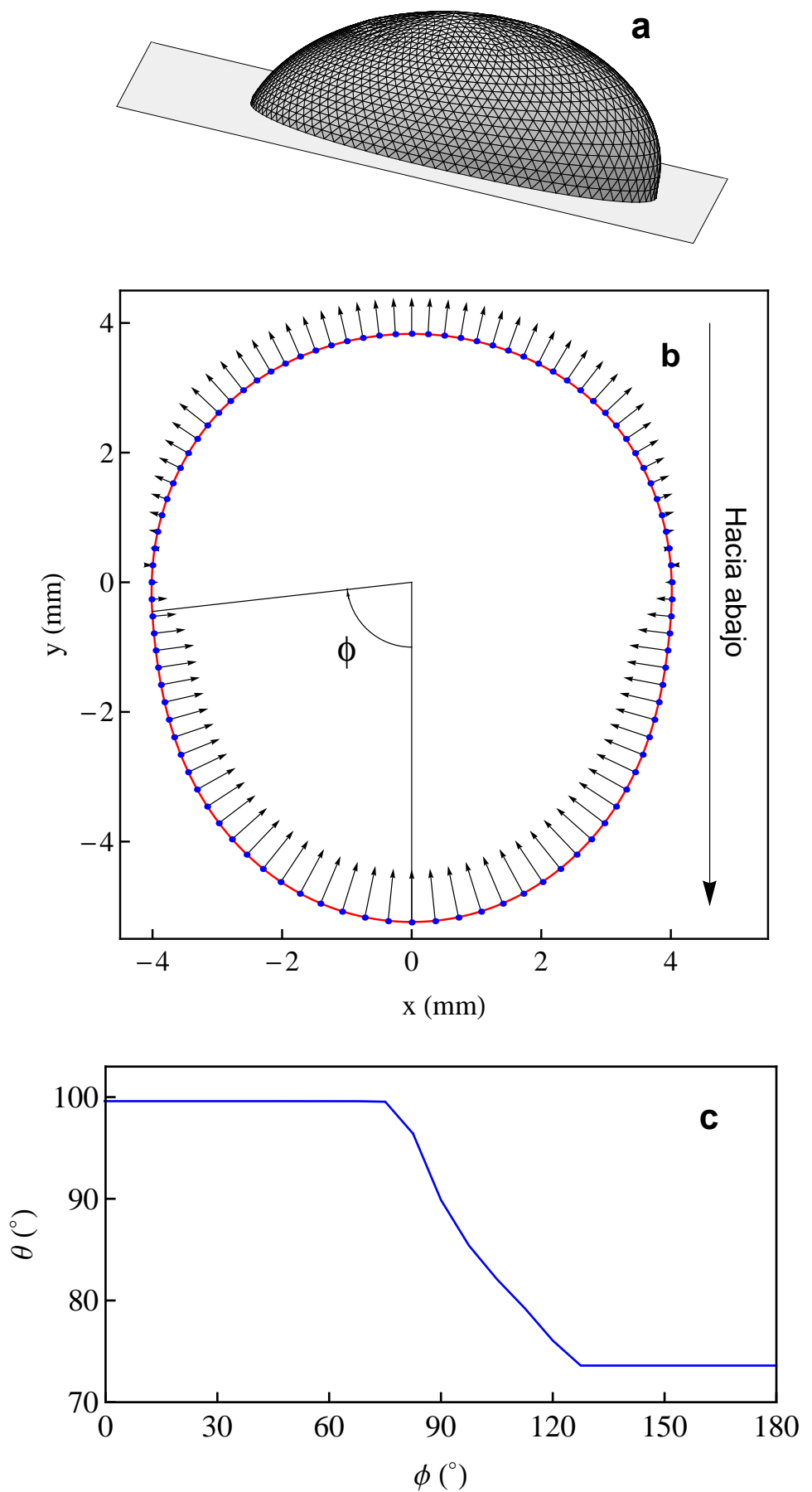

Figura 3.7: Resultados de Surface Evolver para una gota sobre un plano inclinado. a) Forma de la gota. b) Línea de contacto triple mostrando la posición de los vértices y las fuerzas de fricción que actúan sobre ellos (ver texto). c) Ángulos de contacto en función del ángulo $\phi$ definido en b). 
En la figura 3.7 se presentan los resultados de simulación para una gota de agua de volumen $V=0,116 \mathrm{~cm}^{3}$ sobre una superficie inclinada de PCTFE. Se ha considerado $\theta_{\mathrm{av}}=99,6^{\circ}$ y $\theta_{\mathrm{re}}=73,6^{\circ}$ que son los ángulos de avance y retroceso críticos obtenidos por Extrand y Kumagai [54] para agua sobre una superficie inclinada de PCTFE. En esta simulación se ha considerado un ángulo de inclinación de $\alpha=13^{\circ}$ que está cerca de la inclinación crítica para el volumen considerado. Como ya se ha explicado, para simular la manera en que se realizan los experimentos se ha comenzado con una superficie sin inclinar para la cual se ha hecho crecer la gota hasta obtener el volumen deseado. Esto da lugar al hecho de que el ángulo de contacto inicial de la gota es el ángulo de avance. Entonces el plano se inclina lentamente, alcanzando el equilibrio para cada ángulo de inclinación intermedio, hasta la inclinación final. Las medidas se han realizado después de un largo periodo de simulación hasta alcanzar el equilibrio.

La figura 3.7a muestra la forma estable de la gota obtenida utilizando en Surface Evolver el comando gfric en lugar de g (ver capítulo anterior). Como se puede observar la gota ya no es axisimétrica debido al balance entre la fuerza gravitatoria en un plano inclinado y la fuerza retentiva debida a la histéresis del ángulo de contacto. La figura 3.7b muestra la línea de contacto triple, además se han representado las fuerzas de retención $F_{i} / \sigma_{\mathrm{lv}}$ que actúan sobre los vértices. Para mayor claridad se ha escalado $F_{i} / \sigma_{\mathrm{lv}}$ con un factor de 10 por lo que sus unidades son $10^{-1} \mathrm{~mm}$ en lugar de $\mathrm{mm}$. Se señala que estas fuerzas se cancelan en el eje $x$ y dan lugar a una fuerza neta de retención en el eje $y$ del plano inclinado. Esta fuerza retentiva neta compensa el efecto de la gravedad. La figura 3.7c muestra los ángulos de contacto en función del ángulo $\phi$ definido en la figura 3.7b. Se observa que, en la parte delantera de la gota, los ángulos de contacto son iguales a $\theta_{\mathrm{av}}=99,6^{\circ}$ mientras que en la parte posterior los ángulos de contacto son iguales a $\theta_{\text {re }}=73,6^{\circ}$.

La figura 3.8 compara la fuerza de retención total con la contribución de la gravedad para una gota de agua, ahora de volumen $V=0,056 \mathrm{~cm}^{3}$ sobre una superficie inclinada de PCTFE [54]. En la simulación de nuevo se consideran los ángulos de contacto de avance y retroceso obtenidos por Extrand y Kumagai [54], es decir, $\theta_{\mathrm{av}}=99,6^{\circ}$ y $\theta_{\mathrm{re}}=73,6^{\circ}$. En este caso, la simulación se ha realizado comenzando a 


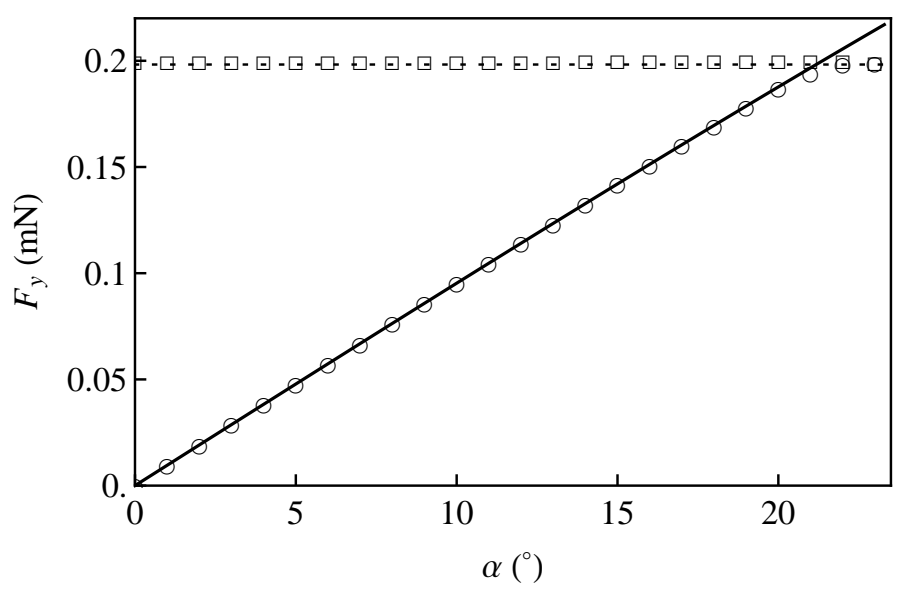

Figura 3.8: Balance de fuerzas para una gota en un plano inclinado. Línea continua: contribución de la gravedad $\rho g V \operatorname{sen} \alpha$. Círculos: fuerza de retención total a lo largo de la dirección de inclinación $F_{y}$. Línea de puntos: Fuerza de retención total máxima estimada a partir de la ecuación (3.3) con $k=2$. Cuadrados: Fuerza de retención total máxima estimada durante la simulación.

partir de una gota axisimétrica con un ángulo de contacto inicial igual a $\theta_{\mathrm{av}}$ y situada en una superficie horizontal $\left(\alpha=0^{\circ}\right)$, a continuación se aumenta el ángulo de inclinación $\alpha$ con pasos de $\Delta \alpha=1^{\circ}$. Para cada $\alpha$ se han realizado un número total de $N=1000$ pasos con el fin de alcanzar el equilibrio. Como se puede observar en la figura 3.8, la fuerza de retención $F_{y}$ es igual, dentro de la precisión numérica de la simulación, al término gravitacional $\rho g V \operatorname{sen} \alpha$ para $\alpha \lesssim 21^{\circ}$, indicando que la gota no se ha movido y que el sistema ha alcanzado el equilibrio. Para $\alpha \gtrsim 21^{\circ}$ la fuerza de retención alcanza su valor máximo y no puede compensar el término gravitatorio. Bajo estas circunstancias la gota se desliza y la simulación no alcanza el equilibrio. En todos los resultados de simulación se obtiene el mismo comportamiento, es decir, $F_{\mathrm{y}}=\rho g V$ sen $\alpha$ para ángulos de inclinación menores que $\alpha_{c}$. Por supuesto, por encima de ángulo crítico $\alpha_{c}$, Surface Evolver no puede describir correctamente la gota deslizante y los resultados presentes son una mera indicación del cambio de comportamiento del sistema.

El valor máximo de la fuerza de retención $F_{y, \max }$ se puede obtener durante un 
proceso de simulación calculando la fuerza máxima de retención para cada vértice de la línea de contacto triple, realizando la suma de todas estas contribuciones, y proyectándola entonces sobre la dirección de inclinación, $y$. Los valores de $F_{y \text {,max }}$ obtenidos para cada ángulo de inclinación se representan con cuadrados en la figura 3.8. Otra estimación de la fuerza de retención máxima se puede realizar considerando la contribución de la gravedad para el ángulo crítico

$$
F_{\mathrm{c}}=\rho g \vee \operatorname{sen} \alpha_{\mathrm{c}}
$$

A partir de la ecuación (3.3) con $k=2$ se obtiene

$$
F_{\mathrm{c}}=2 \sigma_{\mathrm{lv}} \omega\left(\cos \theta_{\mathrm{re}}-\cos \theta_{\mathrm{av}}\right)
$$

donde el valor de $\omega$ se puede obtener a partir de los datos de la simulación. En las simulaciones $\omega$ se mantiene prácticamente constante para $\alpha<\alpha_{\mathrm{c}}$ y así $F_{\mathrm{c}}$ es también constante por debajo del ángulo crítico. En el caso presente se obtiene $F_{\mathrm{c}}=0,198241 \mathrm{mN}$. Este valor se representa en la figura 3.8 (línea de puntos) con un buen acuerdo con los resultados de $F_{y \text {,max }}$. Por otra parte, utilizando este valor en la ecuación (3.5) se obtiene un valor para el ángulo crítico $\alpha_{c}=21,176^{\circ}$, también en buen acuerdo con las observaciones anteriores. A partir de las ecuaciones (3.5) y (3.6), se puede obtener que $\alpha_{c}$ depende del volumen a través de la siguiente expresión,

$$
\operatorname{sen} \alpha_{\mathrm{c}}=\frac{2 \sigma_{\mathrm{IV}} \omega\left(\cos \theta_{\mathrm{re}}-\cos \theta_{\mathrm{av}}\right)}{\rho g V} \approx V^{-2 / 3}
$$

donde se ha supuesto que $\omega \approx V^{1 / 3}$. Además, el volumen mínimo de la gota deslizante se obtiene fácilmente a partir de la ecuación (3.7) tomando $\alpha_{c}=90^{\circ}$. El comportamiento predicho por la ecuación (3.7) coincide con los resultados de la simulación. Es preciso señalar que, en general, en las simulaciones se ha observado que la gota comienza a deslizarse con un ángulo de inclinación ligeramente menor que el obtenido a partir de la ecuación (3.3) con $k=2$. Este hecho puede deberse a inestabilidades numéricas durante el proceso de ejecución del programa.

Los resultados previos demuestran que la ecuación (3.3) con $k=2$ está de acuerdo con los resultados de las simulaciones para inclinaciones próximas a la crítica 
$\alpha_{c}$. Esto indica que una ecuación similar podría ser válida para inclinaciones menores que $\alpha_{\mathrm{c}}$. Se propone

$$
\frac{\rho g V \operatorname{sen} \alpha}{\sigma_{\mathrm{IV}} \omega}=k\left(\cos \theta_{\min }-\cos \theta_{\max }\right)
$$

donde $\theta_{\min }$ y $\theta_{\max }$ son, respectivamente, los ángulos de contacto mínimo y máximo de la gota para un ángulo de inclinación dado. En general, $\theta_{\min }$ y $\theta_{\max }$ dependen de la inclinación, es decir, $\theta_{\min }=\theta_{\min }(\alpha)$ y lo mismo se aplica para $\theta_{\max }$. Es de destacar que la ecuación (3.8) con $k=1$ se puede obtener sustituyendo el área $A$ por la relación $V / \omega$ en la ecuación (3.1). Teniendo en cuenta que para $\alpha<\alpha_{c}$ la fuerza gravitacional $\rho g V \operatorname{sen} \alpha$ equilibra la fuerza de retención total $F_{y}$, la ecuación (3.8) da paso a

$$
\frac{F_{y}}{\sigma_{\mathrm{IV}} \omega}=k\left(\cos \theta_{\min }-\cos \theta_{\max }\right) .
$$

Para probar la ecuación (3.9), en la figura 3.9a se representa la fuerza de retención total adimensional $F_{y} / \sigma_{\mathrm{IV}} \omega$ frente a la diferencia de $\operatorname{cosenos} \Delta \cos =\cos \theta_{\min }-$ $\cos \theta_{\max }$ para el sistema considerado en la figura 3.8. Para $\Delta \cos <\Delta \cos _{\max }=$ $\cos \theta_{\text {re }}-\cos \theta_{\text {av }}$ se obtiene un comportamiento lineal de acuerdo con la ecuación (3.9). En este caso la regresión lineal de los datos arroja una pendiente $k=1,779$. El valor máximo de $\Delta$ cos se alcanza para un ángulo de inclinación ligéramente inferior $\alpha=19^{\circ}$ (ver figura 3.10a). Una vez que se ha alcanzado este valor, $F_{y} / \sigma_{\mathrm{lv}} \omega$ sigue aumentando hasta alcanzar (aproximadamente) el valor dado por la pendiente $k=2$ para la inclinación crítica. Por lo tanto, una vez que se ha alcanzado el valor máximo de $\Delta$ cos, el hecho de aumentar el ángulo de inclinación da paso a un cambio en el factor de la fuerza de retención k para equilibrar el aumento de la fuerza gravitacional.

En este caso para el cual se comienza la simulación con un ángulo de contacto igual a $\theta_{\mathrm{av}}$ se observa que $\theta_{\max }$ se mantiene igual a $\theta_{\mathrm{av}}$ mientras que $\theta_{\min }$ disminuye con la inclinación. Esto se muestra en la figura 3.10a donde se observa que $\theta_{\text {min }}$ decrece linealmente con $\alpha$ hasta que se alcanza $\theta_{\text {re }}$. Este comportamiento lineal se encuentra estrechamente relacionado con la ecuación (3.8). A partir de esta ecuación debería esperarse una relación lineal entre $\cos \theta_{\min } \mathrm{y} \operatorname{sen} \alpha, \sin$ embargo, en este caso, esta relación también se aplica a $\theta_{\min } \mathrm{y} \alpha$, debido a los valores tomados por estos ángulos. Para valores menores de $\theta_{\min }$ o valores mayores de $\alpha$ tienen lugar desviaciones del 

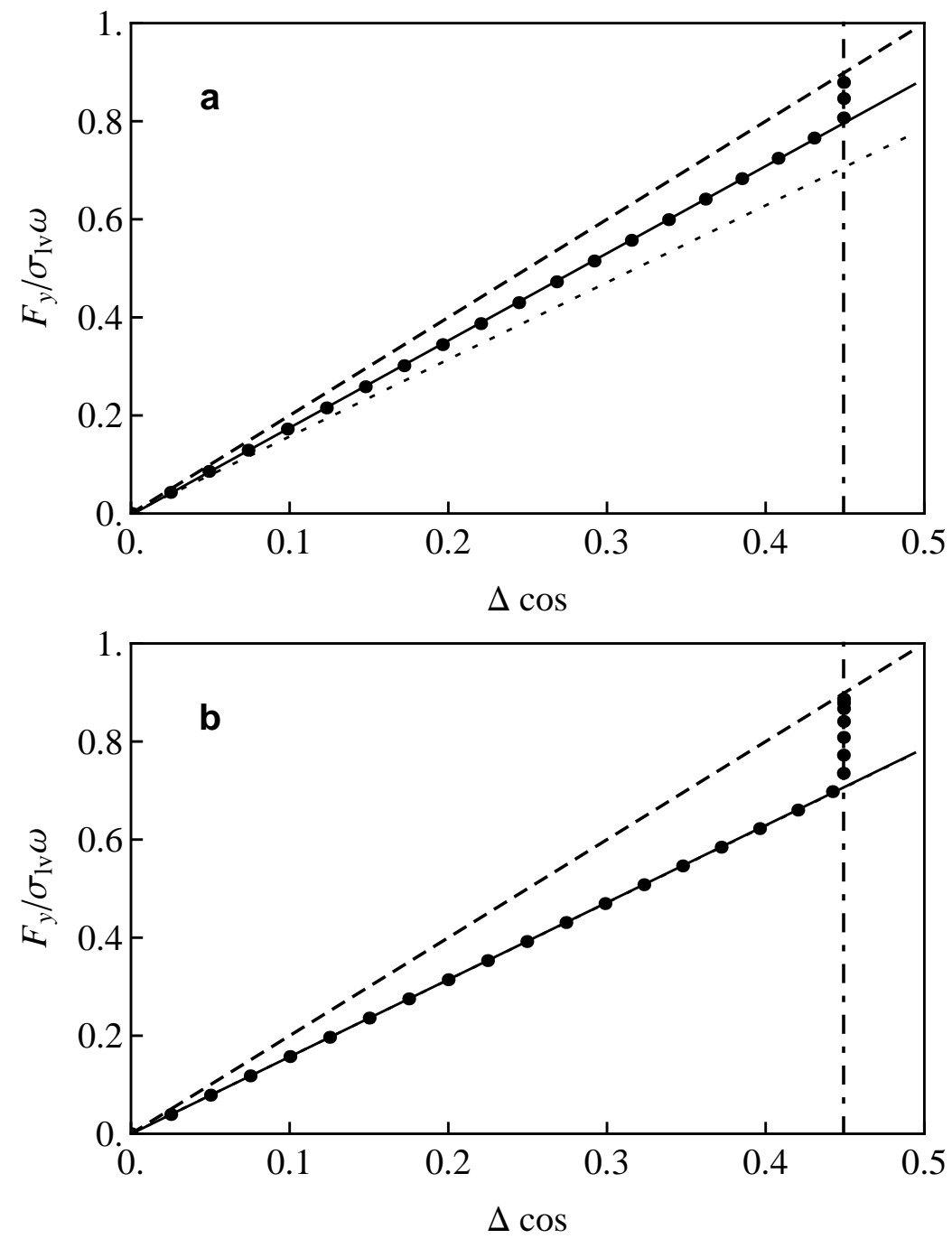

Figura 3.9: Fuerza de retención total adimensional $F_{y} / \sigma_{\mathrm{Iv}} \omega$ frente a la diferencia de $\operatorname{cosenos} \Delta \cos =\cos \theta_{\min }-\cos \theta_{\max }$. a) Ángulo de contacto inicial igual a $\theta_{\mathrm{av}}$. b) Ángulo de contacto inicial igual a $\theta_{Y}$. Los símbolos son datos de la simulación. La línea continua son ajustes lineales de los datos, con pendientes a) $k=1,779 \mathrm{y}$ b) $k=1,573 \approx \pi / 2$. Línea discontinua: pendiente $k=2$. Línea de puntos: pendiente $k=\pi / 2$. La línea vertical punto-raya indica $\Delta \cos _{\max }=\cos \theta_{\mathrm{re}}-\cos \theta_{\mathrm{av}}$. 

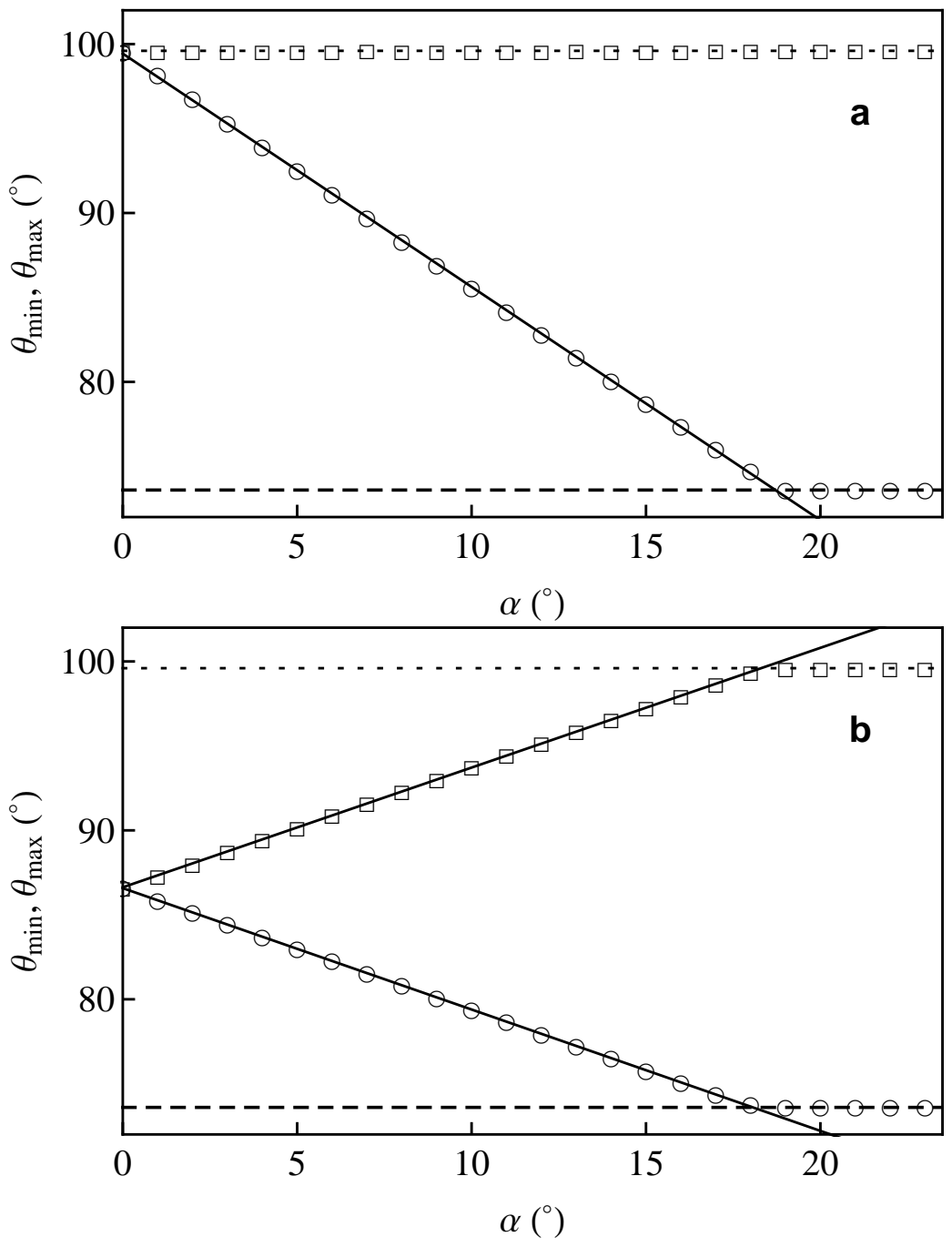

Figura 3.10: $\theta_{\min }$ (círculos) y $\theta_{\max }$ (cuadrados) frente al ángulo de inclinación $\alpha$. a) Ángulo de contacto inicial igual a $\theta_{\mathrm{av}}$. b) Ángulo de contacto inicial igual a $\theta_{Y}$. Los símbolos son datos de la simulación. La línea continua son ajustes lineales de los datos. La línea discontinua y la línea de puntos indican $\theta_{\mathrm{re}}$ y $\theta_{\mathrm{av}}$, respectivamente. 


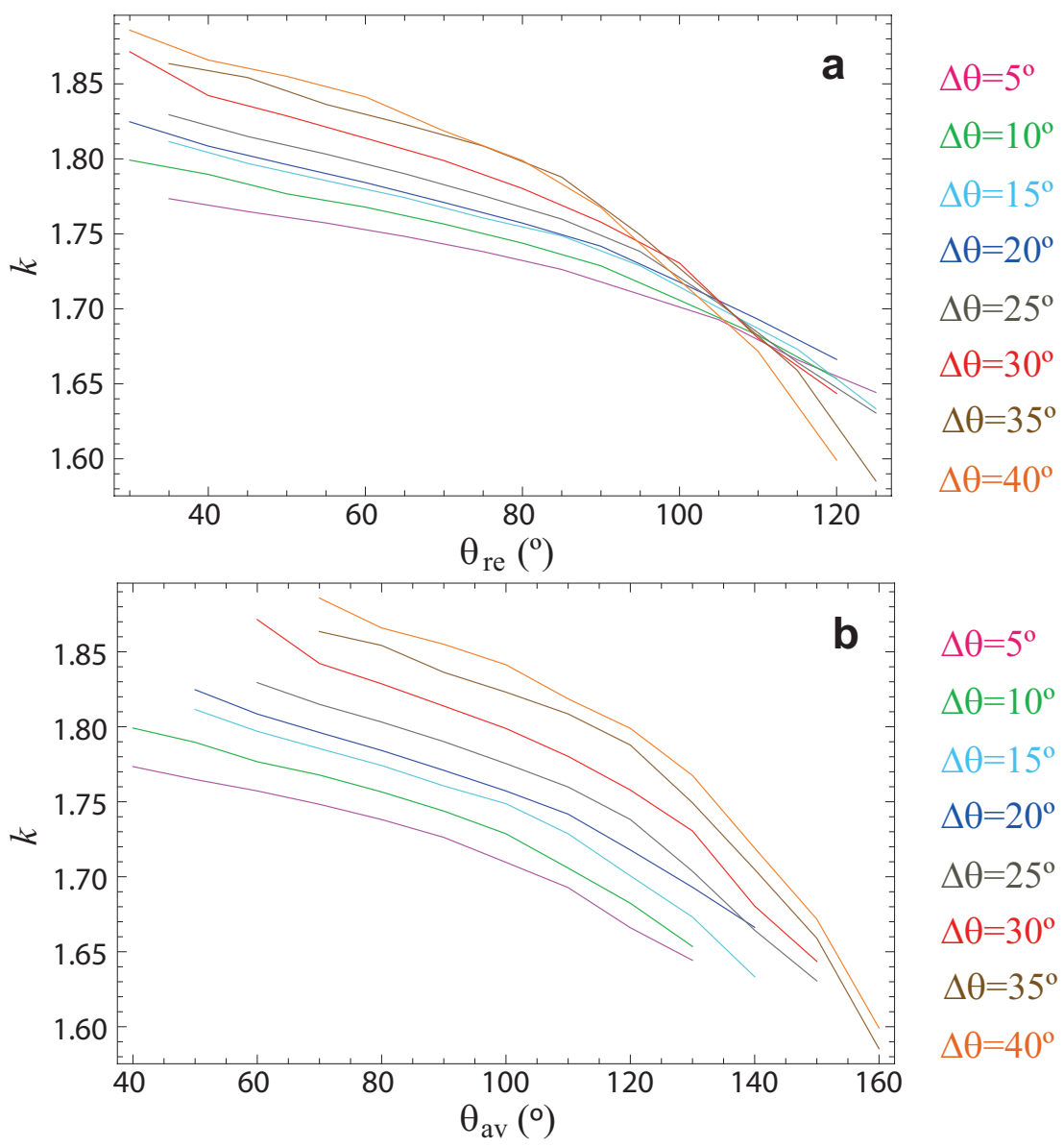

Figura 3.11: Pendiente $k$ frente a) al ángulo de contacto de retroceso $\theta_{\mathrm{re}}, y$ b) al ángulo de contacto de avance $\theta_{\mathrm{av}}$.

comportamiento lineal, como se verá en el siguiente apartado.

Como se termina de ver, la fuerza de retención adimensional $F_{\mathrm{y}} / \sigma_{\mathrm{lv}} \omega$ es proporcional a la diferencia de cosenos $\Delta \cos =\theta_{\min }-\theta_{\max }$ [véase ecuación (3.9)], con una constante de proporcionalidad $k$ que en caso de la figura 3.9a tomaba el valor de $k \simeq 1,779$. Para otros sistemas este comportamiento lineal se mantiene pero con distintos valores de $k$. En la figura 3.11 se recogen los resultados de las simulaciones con Surface Evolver, respecto a los valores de la pendiente $k$ frente al ángulo de contacto de avance (figura 3.11a) y frente al de retroceso (figura 3.11b), para diferentes valores de $\Delta \theta=\theta_{\mathrm{av}}-\theta_{\text {re. }}$ En la figura $3.11 \mathrm{~b}$ se puede apreciar que, para un 


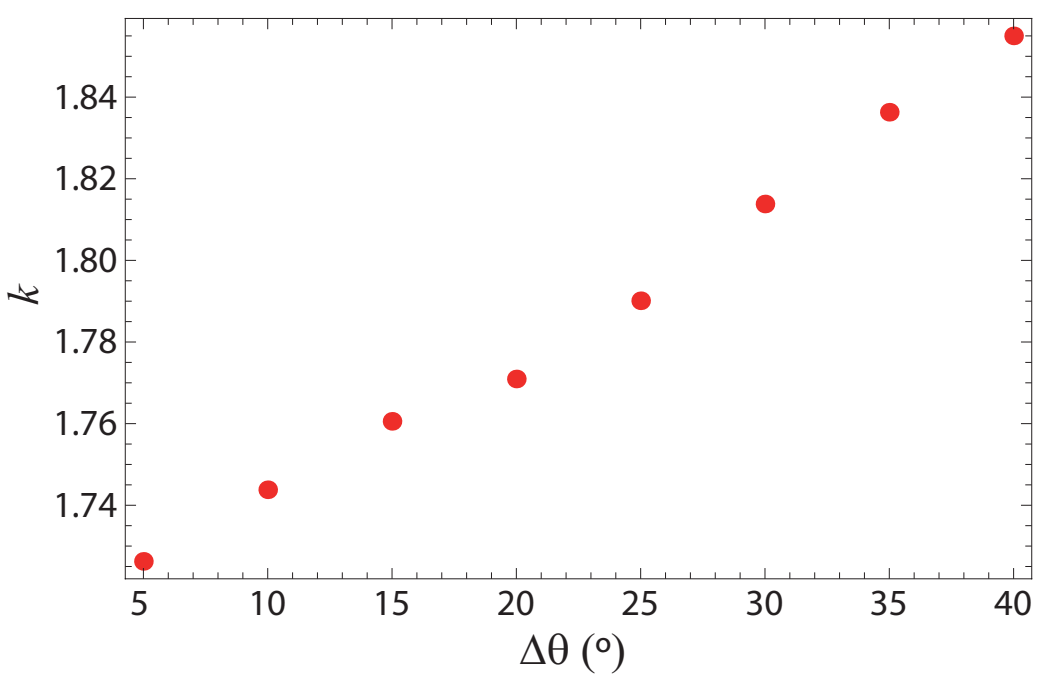

Figura 3.12: Pendiente $k$ frente a la diferencia entre ángulo de contacto de avance y retroceso $\Delta \theta=\theta_{\mathrm{av}}-\theta_{\mathrm{re}}$, para una gota de agua cuando se inclina progresivamente el substrato sobre el que se encuentra, $\operatorname{con} \theta_{\mathrm{av}}=90^{\circ}$.

mismo ángulo de contacto de avance $\theta_{\mathrm{av}}$, las pendientes $k$ van aumentando a medida que aumenta $\Delta \theta$. Esto está relacionado con el hecho de que el ángulo de Young no es el mismo en todas las simulaciones, pues como ya se ha indicado se considera $\theta_{\mathrm{Y}}=\left(\theta_{\mathrm{av}}+\theta_{\mathrm{re}}\right) / 2$ y al ir variando $\Delta \theta$ con $\theta_{\mathrm{av}}$ fijo varía también $\theta_{\mathrm{Y}}$. Por otra parte, en la misma figura 3.11b, se observa que las pendientes correspondientes al mismo valor de $\Delta \theta$ presentan un comportamiento similar, reproduciéndose la tendencia decreciente a medida que aumenta el ángulo de avance; no se observa una pauta tan clara en la figura 3.11a, por lo que se centra el estudio en el ángulo de avance $\theta_{\mathrm{av}}$ para profundizar en estas relaciones.

Este estudio se ciñe ahora a un caso determinado. Para una gota de agua, fijado $\theta_{\mathrm{av}}$, se analiza como varía la pendiente $k$ con otros parámetros del sistema. En la figura 3.12 se presentan los resultados de simulación para $k$, respecto a la diferencia entre el ángulo de contacto de avance y retroceso $\Delta \theta=\theta_{\mathrm{av}}-\theta_{\mathrm{re}}$, para $\theta_{\mathrm{av}}=90^{\circ}$. Se puede apreciar de un modo claro lo expuesto anteriormente, es decir, para un mismo valor de $\theta_{\mathrm{av}}$, las pendientes van aumentando a medida que aumenta $\Delta \theta=\theta_{\mathrm{av}}-\theta_{\mathrm{re}}$. 


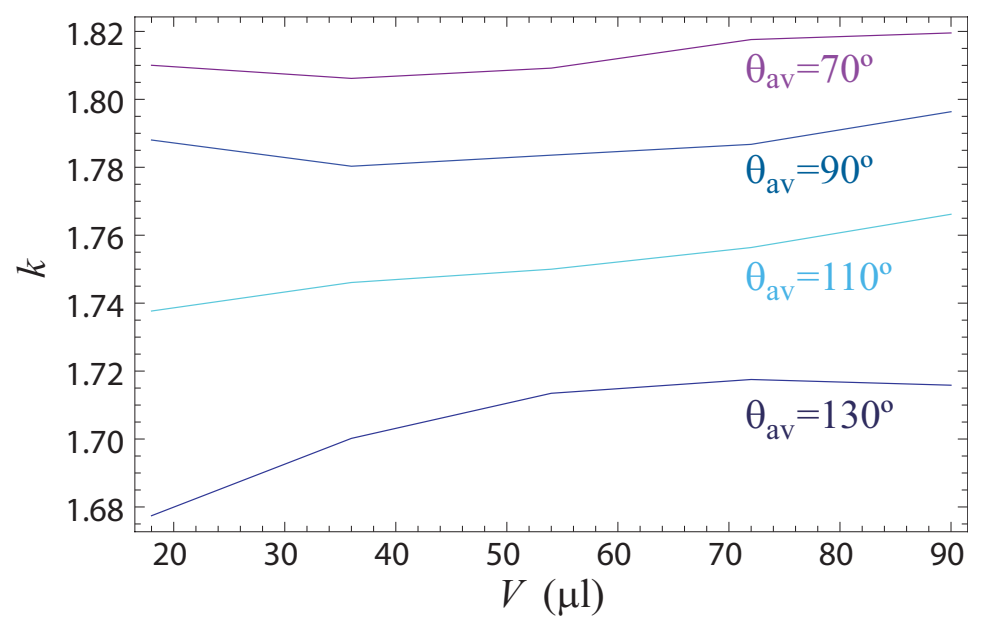

Figura 3.13: Pendiente $k$ frente al volumen. Resultados de simulación con Surface Evolver para una gota de agua con $\Delta \theta=\theta_{\mathrm{av}}-$ $\theta_{\mathrm{re}}=20^{\circ}$ y distintos ángulos de contacto de avance.

En los resultados presentados hasta el momento se ha fijado el volumen de la gota de agua; se verá a continuación qué sucede si se toman volúmenes diferentes de la misma. En la figura 3.13 se ha realizado la representación de los resultados de simulación para las pendientes $k$ frente al volumen, con $\Delta \theta=\theta_{\mathrm{av}}-\theta_{\mathrm{re}}=20^{\circ}$, y distintos ángulos de contacto de avance. De esta gráfica se puede deducir por un lado que, de acuerdo con la figura 3.11b, fijado $\Delta \theta$ el valor de $k$ disminuye al aumentar $\theta_{\mathrm{av}}$ y por otro, que el valor de $k$ permanece prácticamente constante con el volumen para cada valor de $\theta_{\mathrm{av}}$. Esta última afirmación se puede comprobar en la figura 3.14 en la que se han representado conjuntamente todas las rectas $F_{\mathrm{y}} / \sigma_{\mathrm{lv}} \omega$ frente a la diferencia de los cosenos de los ángulos de contacto mínimo y máximo, $\Delta \cos =\cos \theta_{\min }-\cos \theta_{\max }, \operatorname{con} \theta_{\mathrm{av}}-\theta_{\mathrm{re}}=20^{\circ}, \theta_{\mathrm{av}}=130^{\circ}$ y diferentes volúmenes.

\section{Forma de la línea de contacto triple en función de los ángulos de contacto}

La figura 3.15 muestra la evolución con la inclinación de la línea de contacto triple (figura 3.15a) y los ángulos de contacto (figura 3.15b). Para $\alpha=0^{\circ}$ la línea de contacto tiene una forma circular (línea de puntos en la figura 3.15a) y todos los ángulos de contacto son iguales a $\theta_{\mathrm{av}}=99,6^{\circ}$. Para $0^{\circ}<\alpha \lesssim 19^{\circ}$ la parte posterior 


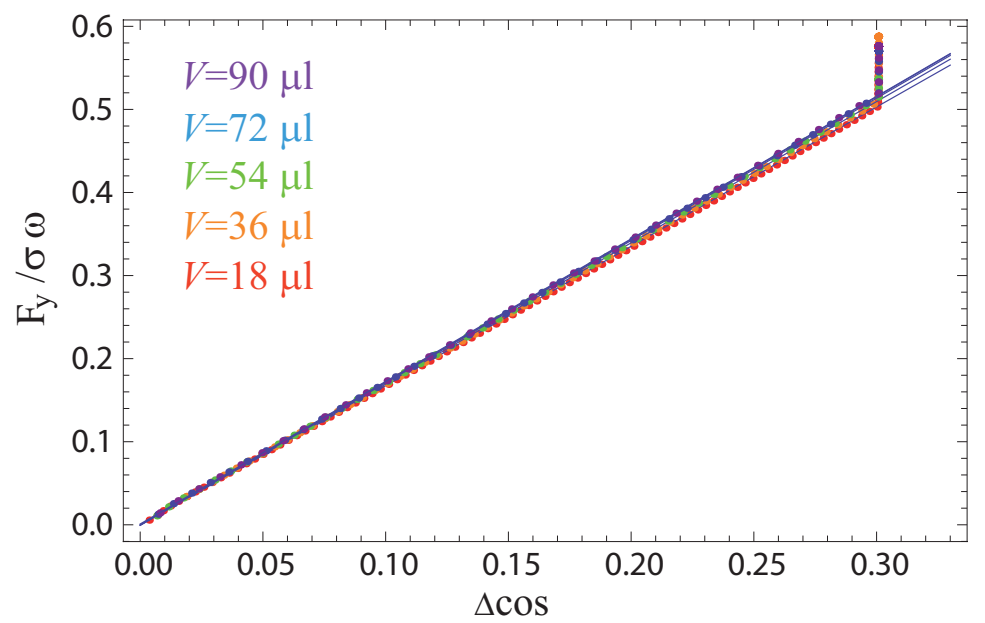

Figura 3.14: Fuerza $F_{\mathrm{y}} / \sigma_{\mathrm{Iv}} \omega$ para una gota de agua, frente a la diferencia de cosenos de los ángulos de contacto $\Delta \cos =\cos \theta_{\min }-$ $\cos \theta_{\max }, \operatorname{con} \theta_{\mathrm{av}}-\theta_{\mathrm{re}}=20^{\circ}, \theta_{\mathrm{av}}=130^{\circ}$ y volúmenes distintos.

de la línea de contacto permanece fija mientras que la parte frontal se desplaza hacia abajo. Esto se muestra en la figura 3.15a para un ángulo de inclinación $\alpha=10^{\circ}$ (línea continua). En la figura 3.15b se muestra (línea continua) el perfil del ángulo de contacto para este caso intermedio y se observa que mientras todos los ángulos de contacto en la parte frontal son iguales a $\theta_{\mathrm{av}}$, los ángulos de contacto de la parte posterior de la línea de contacto triple son aún menores que $\theta_{\text {re }}=73,6^{\circ}$, lo que explica por qué la parte posterior de la línea de contacto permanece fija. Para $\alpha=20^{\circ}$ (línea discontinua) se observa en la figura 3.15b que parte de los ángulos de contacto de la zona posterior han alcanzado el valor de retroceso $\theta_{\text {re }} \mathrm{y}$, de acuerdo con ello, esta parte posterior de la línea de contacto triple se ha desplazado ligeramente hacia abajo (ver figura 3.15a, línea discontinua). Como parte de la línea de contacto está fija aún, la gota permanece en reposo. Para $\alpha=23^{\circ}$ (línea discontinua puntoraya) la gota está ya deslizándose. En este caso se señala que la anchura de la gota llega a ser ligeramente menor que la original y la gota tiende a adquirir la forma considerada por Dussan y Chow $[35,36]$ con una línea de contacto formada por dos arcos semicirculares unidos por dos lados paralelos (figura 3.15a) donde los ángulos de contacto para el arco de la parte frontal son iguales a $\theta_{a v}$ y los del arco posterior son 

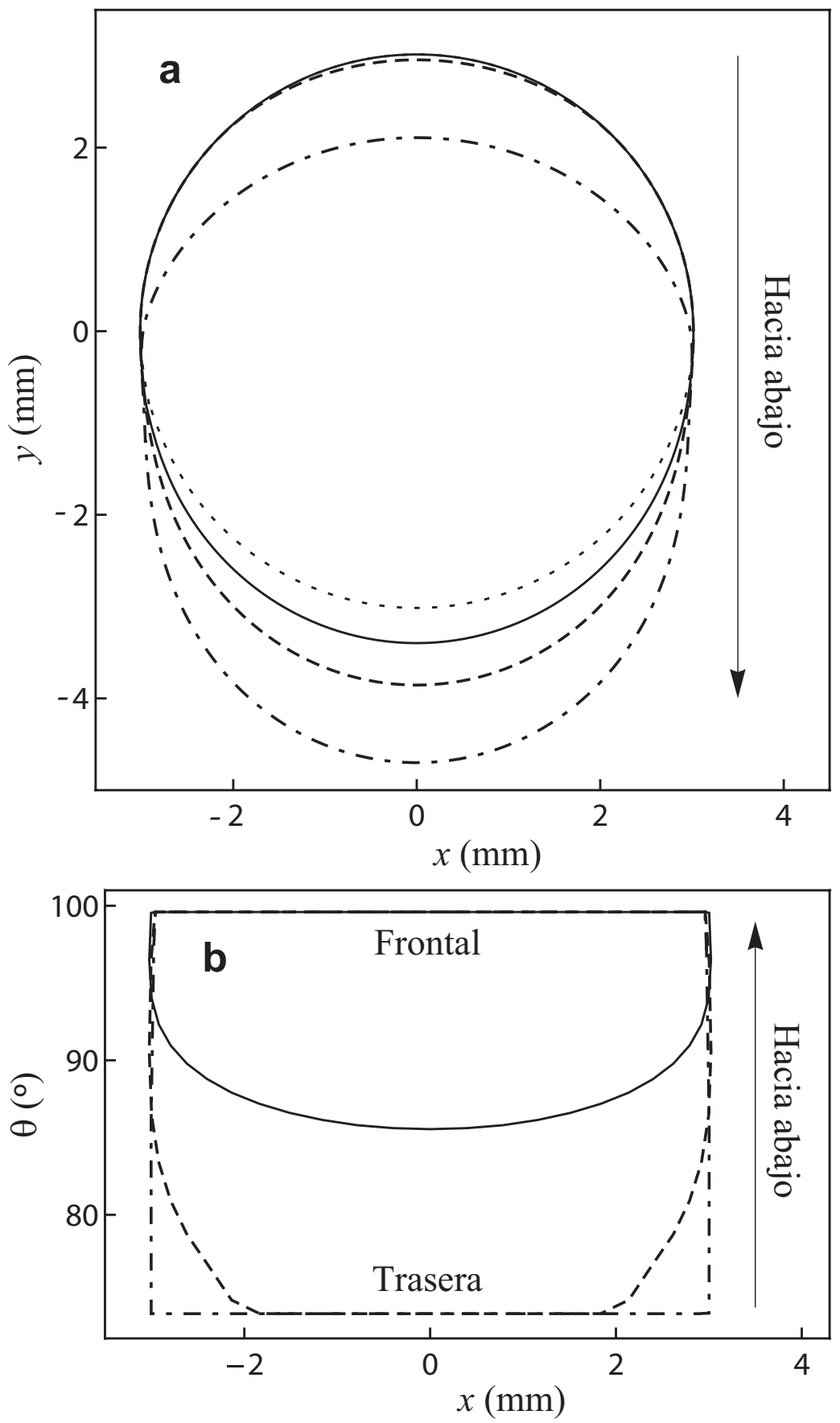

Figura 3.15: Resultados de Surface Evolver para gotas con diferentes ángulos de inclinación a con un ángulo de contacto inicial igual a $\theta_{\mathrm{av}}$. a) Línea de contacto triple. b) Ángulos de contacto en función de la coordenada x perpendicular a la inclinación. Línea de puntos: $\alpha=0^{\circ}$, línea continua: $\alpha=10^{\circ}$, línea discontinua: $\alpha=20^{\circ}$, línea punto-raya: $\alpha=23^{\circ}$. 
iguales a $\theta_{\text {re }}$ (figura 3.15b). A pesar del hecho de que los resultados para $\alpha=23^{\circ}$ están de acuerdo con trabajos previos, hay que tener en cuenta que, como se ha mencionado anteriormente, las simulaciones no pueden describir de modo correcto una gota que se desliza por completo.

\subsection{2. Ángulo de contacto inicial igual a $\theta_{Y}$}

Algunos trabajos recientes han considerado procedimientos de inyección/succión que permiten preparar gotas con diferentes ángulos iniciales $[29,45,46,66]$, por lo tanto se puede considerar un experimento de inclinación del plano con unas condiciones iniciales predeterminadas. Como se verá a continuación para un caso con ángulo de contacto $\theta$ igual a $\theta_{Y}$, las condiciones iniciales de la gota van a ser determinantes en los resultados del proceso de inclinación. Al igual que en el subapartado anterior, se expone en primer lugar el comportamiento de las fuerzas de retención y posteriormente el valor de la línea de contacto triple en función de los ángulos de contacto.

\section{Fuerzas de retención}

Se considera el mismo sistema de la figura 3.8 pero con una gota inicial axisimétrica con ángulo de contacto igual a $\theta_{Y}$. Un análisis como el llevado a cabo en la figura 3.8 muestra que el término gravitacional $\rho g V \operatorname{sen} \alpha$ se equilibra con la fuerza de retención $F_{y}$ para $\alpha \lesssim 23^{\circ}$. Para $\alpha \gtrsim 23^{\circ}$ la gota se desliza por completo y la simulación no puede equilibrarse. Además, de las ecuaciones (3.5) y (3.6) se obtiene $\alpha_{\mathrm{c}}=23,360^{\circ}$ y $F_{\mathrm{c}}=0,217592 \mathrm{mN}$. Comparando con el caso anterior para el cual $\alpha_{\mathrm{c}}=21,176^{\circ}$ y $F_{\mathrm{c}}=0,198241 \mathrm{mN}$, es de destacar que en ese caso el ángulo de contacto inicial mayor implica una anchura de la gota menor y por lo tanto, ángulo crítico y fuerza de retención menores.

La figura 3.9b muestra la fuerza de retención total adimensional $F_{y} / \sigma_{\mathrm{lv}} \omega$ frente a la diferencia de cosenos $\Delta \cos$ para una situación con un ángulo inicial $\theta_{Y}$. Del mismo modo que en la figura 3.9a, en este caso también se obtiene un comportamiento lineal para $\Delta \cos <\Delta \cos _{\max }$. Sin embargo, en este caso un ajuste lineal de los datos 
proporciona una pendiente $k=1,573 \approx \pi / 2$, que está de acuerdo con los resultados de elementos finitos de Brown el at. para una línea de contacto triple triple fija y circular [33]. Se esperaba esta coincidencia, ya que en la simulación los vértices que pertenecen a la línea de contacto triple permanecen fijos si sus ángulos de contacto $\theta_{i}$ satisfacen $\theta_{\text {re }}<\theta_{i}<\theta_{\mathrm{av}}$. Por lo tanto, el hecho de comenzar con una línea de contacto circular para $\theta=\theta_{Y}$ implica que la línea triple permanece circular hasta que $\theta_{\max }=\theta_{\mathrm{av}} \circ \theta_{\min }=\theta_{\mathrm{re}}$. Estas dos condiciones se alcanzan aproximadamente para el mismo ángulo de inclinación $\left(\alpha \approx 18^{\circ}\right)$ como puede verse en la figura 3.10b donde puede observarse también que $\theta_{\min }\left(\theta_{\max }\right)$ disminuye (aumenta) linealmente con $\alpha$ hasta alcanzar $\theta_{\text {re }}\left(\theta_{\mathrm{av}}\right)$. El valor máximo de $\Delta$ cos se alcanza también para $\alpha \approx 18^{\circ}$. Para $\alpha>18^{\circ}, \Delta \cos$ permanece igual a $\Delta \cos _{\max }$ mientras que $F_{y} / \sigma_{\mathrm{lv}} \omega$ se aproxima a su valor máximo dado por la pendiente $k=2$ (véase figura 3.9b) para el ángulo de inclinación crítico $\alpha \approx 23^{\circ}$.

Como se ha mencionado anteriormente, la relación lineal obtenida para la evolución de los ángulos de contacto máximo y mínimo con la inclinación (para $\Delta$ cos $<$ $\Delta \cos _{\max }$ ) está asociada a la relación lineal entre sen $\alpha \mathrm{y} \cos \theta_{\min } \mathrm{O} \cos \theta_{\max }$. En particular, a partir de la ecuación (3.8), se propone

$$
\frac{\rho g \vee \operatorname{sen} \alpha}{\sigma_{\mathrm{IV}} \omega}=k_{1}\left(\cos \theta_{\min }-c_{1}\right)
$$

para $\theta_{\min }$, siendo $k_{1}$ y $c_{1}$ constantes. Análogamente, también se propone

$$
\frac{\rho g V \operatorname{sen} \alpha}{\sigma_{\mathrm{IV}} \omega}=-k_{2}\left(\cos \theta_{\max }-c_{2}\right)
$$

para $\theta_{\max }$. Comparando con la ecuación (3.8) se obtiene:

$$
c_{1}=c_{2}=\cos \theta_{\text {ini }}
$$

y

$$
\frac{1}{k}=\frac{1}{k_{1}}+\frac{1}{k_{2}}
$$

En el presente caso un ajuste lineal de los datos permite obtener $c_{1}=0,05955$, $c_{2}=0,05940$, muy cerca de $\cos \theta_{Y}=0,05931$, y $k_{1}=k_{2}=3,157$ que a partir de la ecuación (3.13) da $k=1,5785$, muy próximo a $\pi / 2$ y a la pendiente $k=1,573$ obtenida en la figura $3.9 \mathrm{~b}$. En el caso anterior donde $\theta_{\max }=\theta_{\text {ini }}=\theta_{\mathrm{av}} \mathrm{y}$ por lo tanto 
$k_{2} \rightarrow \infty$ se obtiene $c_{1}=-0,1650 \approx \cos \theta_{\mathrm{av}}=-0,1668$ y $k_{1}=k=1,786$ también muy próximo al valor $k=1,779$ obtenido en la figura 3.9a.

\section{Forma de la línea de contacto triple en función de los ángulos de contacto}

La figura 3.16 muestra la evolución con el ángulo de inclinación de la línea de contacto triple (figura 3.16a) y de los ángulos de contacto (figura 3.16b). Para $\alpha=0^{\circ}$ la línea de contacto tiene forma circular (línea de puntos en la figura 3.16a) y todos los ángulos de contacto son iguales a $\theta_{Y}=86,6^{\circ}$ (línea de puntos en la figura 3.16b). Para $0^{\circ}<\alpha \lesssim 18^{\circ}$ toda la línea de contacto permanece fija ya que todos los ángulos de contacto pertenecen al intervalo $\theta_{\text {re }}<\theta<\theta_{\mathrm{av}}$. Como se muestra en la figura $3.16 \mathrm{~b}$ para $\alpha=10^{\circ}$ (línea continua) y $\alpha=18^{\circ}$ (línea punto-puntoraya), al aumentar la inclinación se produce una distribución gradual de los ángulos de contacto de modo que los que se encuentran en la parte posterior disminuyen hasta alcanzar $\theta_{\text {re }}$ y los de la parte frontal aumentan hasta alcanzar $\theta_{\mathrm{av}}$, de acuerdo con los resultados presentados en la figura 3.10b. Para $18^{\circ} \lesssim \alpha \lesssim 23^{\circ}$ algunos de los ángulos de contacto de la zona posterior han alcanzado el valor de retroceso $\theta_{\text {re }} \mathrm{y}$ esta parte de la línea de contacto se desplaza hacia abajo; de modo análogo, algunos de los ángulos de contacto de la zona delantera han alcanzado el valor de avance $\theta_{\text {av }}$ y esta parte se desplaza también hacia abajo, pero la gota permanece en reposo. Este desplazamiento se muestra en la figura 3.16a para $\alpha=21^{\circ}$ (línea continua) y $\alpha=23^{\circ}$ (línea discontinua). Para $\alpha=25^{\circ}$ (figura 3.16, línea continua punto-raya) la gota está ya deslizándose, todos los ángulos de contacto de la parte frontal son iguales a $\theta_{\mathrm{av}} \mathrm{y}$ todos los ángulos de contacto de la parte posterior son iguales a $\theta_{\text {re }}$, y se reduce el ancho de la gota.

Para concluir, en este apartado se ha visto como la elección del ángulo de contacto inicial de la gota tiene un efecto relevante en los resultados obtenidos. Son posibles otras elecciones como un ángulo inicial igual a $\theta_{\text {re }}[29,46]$. En este caso los resultados de simulación, como se verá más adelante, revelan que la parte frontal de la línea de contacto se sujeta inicialmente mientras que la parte posterior se desplaza. Mientras que la parte frontal de la línea de contacto permanece sujeta los ángulos de contacto frontales crecen hasta alcanzar $\theta_{\mathrm{av}}$, de acuerdo con resultados recientes [29]. 

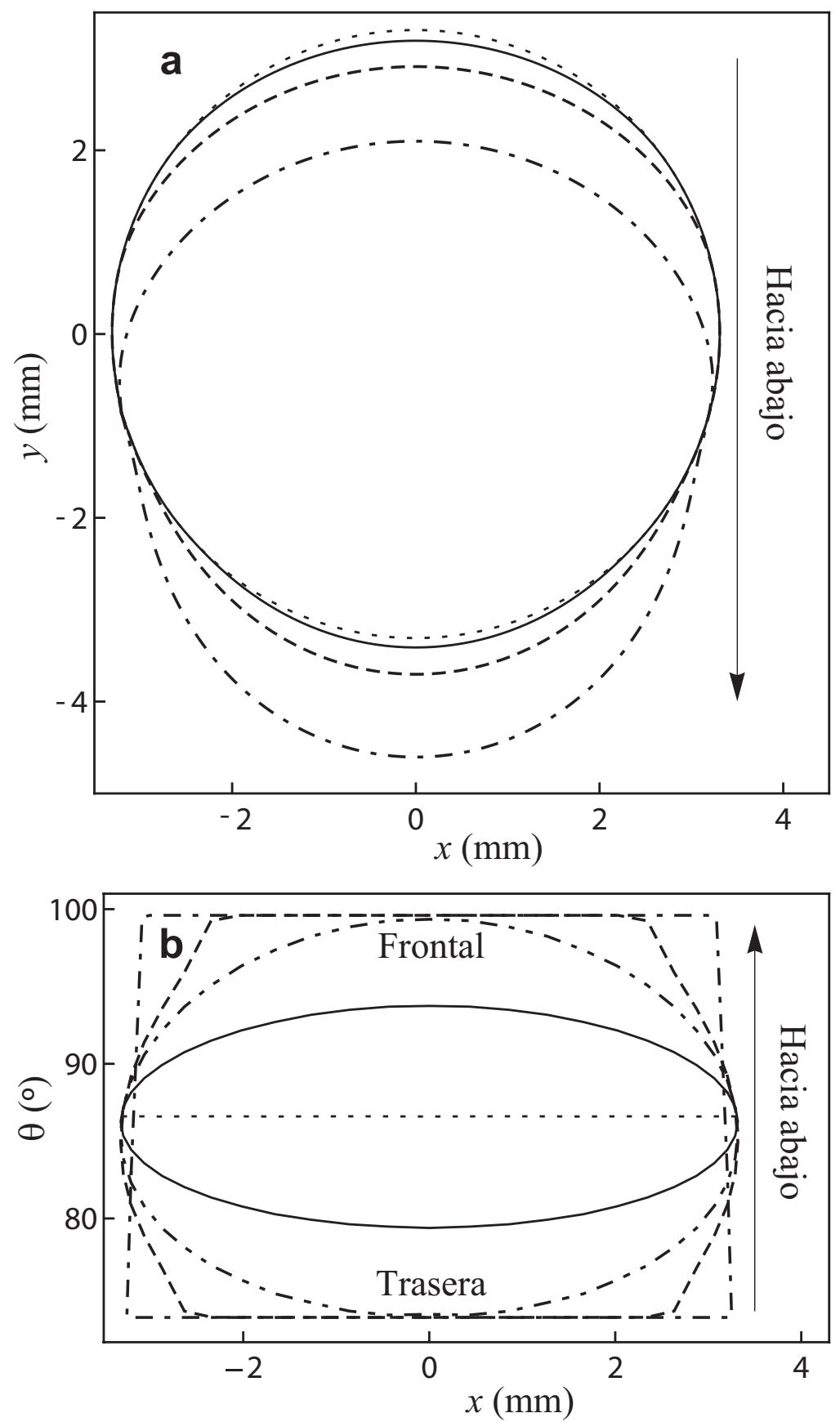

Figura 3.16: Resultados de Surface Evolver para gotas con diferentes ángulos de inclinación a y un ángulo de contacto inicial igual a $\theta_{Y}$. a) Línea de contacto triple. Línea de puntos: $\alpha \leq 18^{\circ}$, línea continua: $\alpha=21^{\circ}$, línea discontinua: $\alpha=23^{\circ}$, línea punto-raya: $\alpha=25^{\circ}$. b) Ángulos de contacto en función de la coordenada $x$ perpendicular a la inclinación. Línea de puntos: $\alpha=0^{\circ}$, línea continua: $\alpha=10^{\circ}$, línea punto-raya: $\alpha=18^{\circ}$, línea discontinua: $\alpha=21^{\circ}$, línea punto-raya: $\alpha=25^{\circ}$. 


\subsection{Comparación con datos experimentales}

El propósito de este apartado es comparar los resultados de simulación con datos experimentales de la evolución de los ángulos de contacto máximo y mínimo durante el proceso de inclinación. En particular se comparan los resultados con las medidas de Xu et al. [67] para una aleación fundida de $\mathrm{Sn}-3,0$ wt. \% Ag-0,5 wt. \%Cu (un conocido material de soldadura libre de plomo) sobre una superficie inclinada de cobre libre de oxígeno para un intervalo de temperatura de 490 - $565 \mathrm{~K}$.

De acuerdo con los datos de Xu et al. [67], se considera una muestra de soldadura de $0,72 \mathrm{~g}$ con un ángulo de contacto inicial $\theta=60,9^{\circ}$, un ángulo de contacto de avance $\theta_{\mathrm{av}}=66,8^{\circ}$, y un ángulo de contacto de retroceso $\theta_{\mathrm{re}}=16,8^{\circ}$. Para este sistema, siguiendo los datos de Moser et al. [68] se considera asimismo una densidad de $\rho=7,1 \mathrm{~g} / \mathrm{cm}^{3}$ y una tensión superficial $\sigma_{\mathrm{lv}}=540 \mathrm{mN} / \mathrm{m}$. Antes de analizar los resultados de simulación hay que señalar que, en contraste con los resultados del apartado previo, éste es un sistema con una histéresis pronunciada en la cual el ángulo de contacto inicial no es ni $\theta_{\mathrm{av}}$ ni $\theta_{Y}$. Consecuentemente, se esperan algunas diferencias con los resultados presentados anteriormente.

La figura 3.17a muestra la evolución de $\theta_{\min }$ y $\theta_{\max }$ con el ángulo de inclinación $\alpha$. En general, se obtiene un ajuste excelente entre los datos experimentales y los procedentes de simulación, las principales diferencias se deben a fluctuaciones experimentales. Comparando con los resultados del apartado anterior, en esta situación actual de histéresis grande, la variación de los ángulos de contacto con la inclinación ya no es lineal. Esto se debe al hecho de que en este caso el término de cosenos en las ecuaciones (3.10) y (3.11) no puede ajustarse a una recta. En cambio, si se considera $\cos \theta_{\min } \mathrm{y} \cos \theta_{\max }$, se recupera un comportamiento lineal definido a trozos, como se muestra en la figura $3.17 b$. Se destaca aquí que a partir de las ecuaciones (3.10) y (3.11) podrían esperarse relaciones lineales entre cosenos de los ángulos de contacto y el seno del ángulo de inclinación pero en la situación presente sen $\alpha \approx \alpha$ y, por claridad, se utiliza el ángulo de inclinación.

Se han identificado cuatro pasos en la evolución del ángulo de contacto presentada en la figura 3.17. 

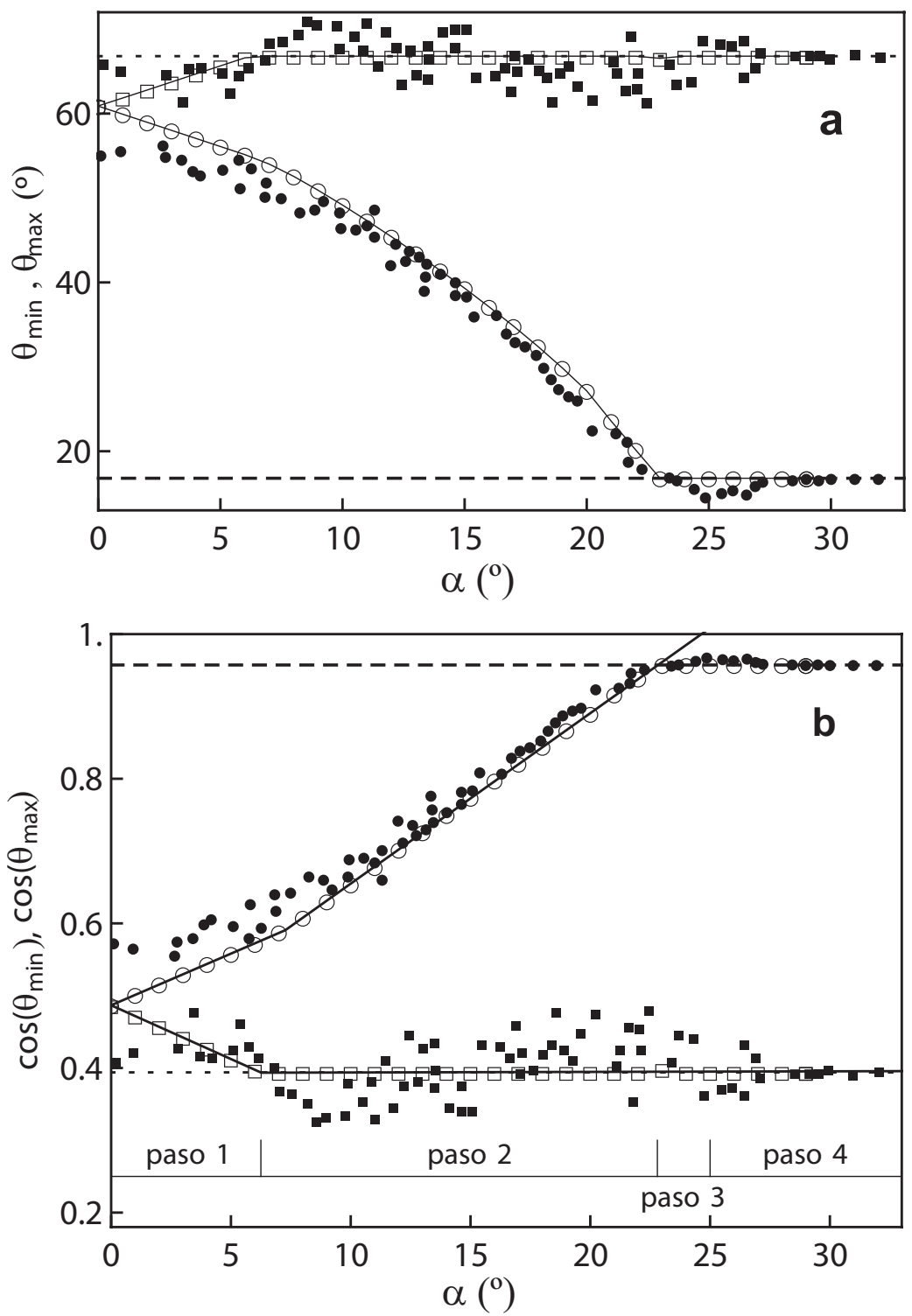

Figura 3.17: Comparación entre los resultados de la simulación y los datos experimentales para $\mathrm{Sn}-\mathrm{Ag}$-Cu fundido sobre una substrato inclinado de cobre. a) $\theta_{\min }$ (círculos) y $\theta_{\max }$ (cuadrados) frente al ángulo de inclinación $\alpha$. Las líneas continuas son guías para la observación. Las líneas discontinuas y las líneas de puntos indican $\theta_{\text {re }} y \theta_{\text {av }}$ respectivamente. b) $\cos \theta_{\min }$ (círculos) y $\cos \theta_{\max }$ (cuadrados) frente al ángulo de inclinación $\alpha$. Las líneas continuas son ajustes lineales de los datos. Las líneas discontinuas y las líneas de puntos indican $\cos \theta_{\mathrm{re}} y \cos \theta_{\mathrm{av}}$ respectivamente. En ambas figuras los símbolos rellenos son los datos experimentales [67]. 
1. $0<\alpha \lesssim 6,3^{\circ}$ : En este caso $\theta_{\min }>\theta_{\text {re }}$ y $\theta_{\max }<\theta_{\mathrm{av}}, \mathrm{y}$, consecuentemente, la línea de contacto triple permanece fija, con un perfil circular. $\cos \theta_{\max }$ decrece linealmente $\operatorname{con} \alpha$ hasta que alcanza $\cos \theta_{\mathrm{av}} \cdot \cos \theta_{\min }$ crece linealmente $\operatorname{con} \alpha$. El comportamiento del sistema es similar al primer paso de la figura 3.10b.

2. $6,3^{\circ} \lesssim \alpha \lesssim 22,8^{\circ}$ : Ya que $\theta_{\max }=\theta_{\mathrm{av}}$, la parte frontal de la gota se desplaza hacia abajo mientras que la parte posterior permanece fija. La forma de la gota se vuelve alargada. Es de destacar que para $\alpha \approx 7,3^{\circ}$ la pendiente de $\cos \theta_{\text {min }}$ cambia y el comportamiento del sistema se torna similar al presentado en la figura 3.10a. Desde $\alpha \approx 7,3^{\circ}, \cos \theta_{\min }$ aumenta linealmente hasta alcanzar $\cos \theta_{\text {re }}$ para $\alpha \approx 22,8^{\circ}$.

3. $22,8^{\circ} \lesssim \alpha \lesssim 25^{\circ}$ : Ahora $\theta_{\min }=\theta_{\text {re }}$ y parte de la línea de contacto posterior se desplaza. La gota permanece estática.

4. $\alpha \geq 25^{\circ}$ : Para $\alpha \approx 25^{\circ}$ se alcanza el ángulo de inclinación crítico y la gota se desliza por completo. La simulación no puede equilibrarse y se vuelve inestable.

Los ángulos de inclinación crítica $\alpha_{\mathrm{d}} \approx 6,3^{\circ} \mathrm{y} \alpha_{\mathrm{u}} \approx 22,8^{\circ}$ indican, respectivamente, donde tiene lugar el movimiento inminente de las partes delantera (downhill) y trasera (uphill) de la línea de contacto. Estos ángulos, junto con el ángulo de inclinación crítica $\alpha_{\mathrm{c}} \approx 25^{\circ}$ determinan los cuatro pasos en la evolución del sistema. Estos pasos revelan una situación híbrida entre las dos situaciones consideradas en el apartado previo. El sistema se comporta primero como en la figura 3.10b y posteriormente como en la figura 3.10a. Un comportamiento similar ha sido obtenido por Berejnov y Thorne para gotas de agua sobre superficies de silicio planas [69].

Es de destacar que se han encontrado algunas diferencias con la descripción de la evolución de los ángulos de contacto realizada por Xu et al. [67] para sus resultados experimentales en este sistema. En particular estos autores encuentran un ángulo de inclinación crítico $\alpha_{\mathrm{c}} \approx 28^{\circ}$ para el rango de temperatura considerado. Además, como se puede observar en la figura 3.17, los valores iniciales experimentales de $\theta_{\max }$ y $\theta_{\min }$ no son iguales, indicando que la línea de contacto inicial no es completamente circular. A pesar de ello, es preciso destacar que el comportamiento global obtenido a partir de la simulación es muy similar a sus resultados. 
Finalmente, nos gustaría destacar que un trabajo reciente [46] que presenta un nuevo método para evaluar el ángulo de contacto más estable de una gota mediante experimentos de inclinación de placas está basado en el análisis de la dependencia sobre el ángulo de contacto inicial de $\alpha_{\mathrm{d}} \mathrm{y} \alpha_{\mathrm{u}}$. El método de simulación del presente trabajo puede incluso utilizarse para tal análisis como se verá más adelante.

\subsection{Análisis de la deformación de gotas sobre super- ficies inclinadas}

A lo largo de este capítulo se ha visto como una gota sobre una superficie inclinada se mantiene adherida debido a un cambio de forma. En este apartado se va a analizar este cambio de forma centrando la atención en la base de la gota.

Puesto que los cambios en la base de la gotas son mayores cuando $\theta_{\mathrm{ini}}=\theta_{\mathrm{av}}$ se centra el estudio a este caso particular. Todos los resultados de este apartado se han obtenido para gotas de agua.

\subsubsection{Relación de aspecto $\beta$ frente a la diferencia de cosenos $\cos \theta_{\min }-\cos \theta_{\max }$}

La relación de aspecto $\beta$ se define como el cociente entre la longitud de la base de la gota $2 L$ y su anchura $2 \omega$ (véase la figura 3.2), es decir,

$$
\beta=\frac{L}{\omega} .
$$

Si partimos de una gota con base circular y ángulo de contacto igual al de avance, situada sobre un substrato horizontal, como se ha visto en la figura 3.15, a medida que el substrato se inclina $L$ aumenta con $\omega=$ cte $y$, por lo tanto, $\beta$ también se incrementa.

En los resultados de simulación se ha observado que para valores de $\theta_{\text {av }}$ comprendidos entre $90^{\circ}$ y $120^{\circ}, \beta$ tiene un comportamiento lineal con pendiente $\hat{k}$ respecto a la diferencia de $\operatorname{cosenos} \Delta \cos =\cos \theta_{\min }-\cos \theta_{\max }$, tal y como se muestra en la 


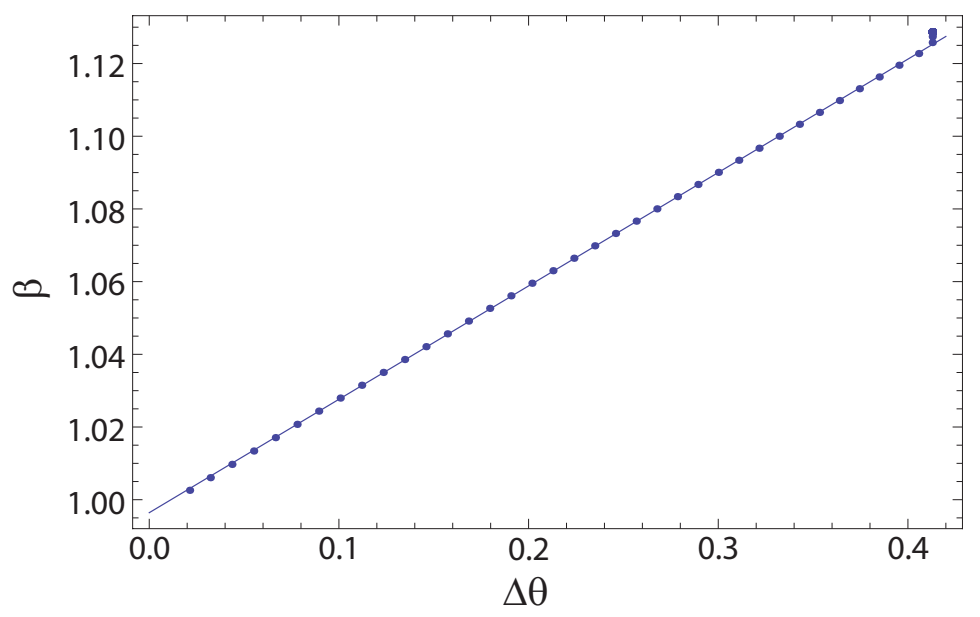

Figura 3.18: Relación de aspecto $\beta$ frente $\Delta \cos$, para $\theta_{\mathrm{re}}-\theta_{\mathrm{av}}=$ $25^{\circ}$, con $\theta_{\mathrm{av}}=120^{\circ}$. Puntos: resultado de simulación con Surface Evolver. Línea: ajuste por mínimos cuadrados de todos los datos.

figura 3.18 para $\theta_{\mathrm{av}}=120^{\circ}$, y $\Delta \theta=\theta_{\mathrm{re}}-\theta_{\mathrm{av}}=25^{\circ}$, es decir,

$$
\beta \simeq 1+\hat{k}\left(\cos \theta_{\min }-\cos \theta_{\max }\right)
$$

Fuera de este intervalo, el comportamiento lineal sólo se alcanza para valores pequeños de $\Delta$ cos, como se puede observar en la figura 3.19 .

En la figura 3.20 se representan los valores obtenidos para $\hat{k}$ frente a $\theta_{\text {re }}$ (figura 3.20a) y frente a $\theta_{\mathrm{av}}$ (figura 3.20b) para distintos valores de $\Delta \theta=\theta_{\mathrm{re}}-\theta_{\mathrm{av}}$. Como puede observarse en la figura 3.20b, la representación en términos de $\theta_{a v}$ da lugar a un comportamiento similar para todos los valores de $\Delta \theta$ considerados, obteniendo valores mínimos para $\hat{k}$ (y por tanto mínimas deformaciones) en el intervalo $80^{\circ}<\theta_{\mathrm{av}}<110^{\circ}$. La representación de $\hat{k}$ en términos de $\theta_{\text {re }}$ (figura 3.20a) no es tan adecuada pues en este caso los valores mínimos de $\hat{k}$ dependen del $\Delta \theta$ considerado.

Para ver la influencia del volumen en la deformación de la gota, se realizan las simulaciones anteriores para diferentes volúmenes de las gotas de agua, manteniendo $\Delta \theta=\theta_{\mathrm{av}}-\theta_{\mathrm{re}}=20^{\circ}$ y considerando distintos ángulos de contacto de avance. Los resultados obtenidos para $\hat{k}$ se presentan en la figura 3.21 donde se observa que, fijado un ángulo de avance, la pendiente $\hat{k}$ permanece prácticamente constante 


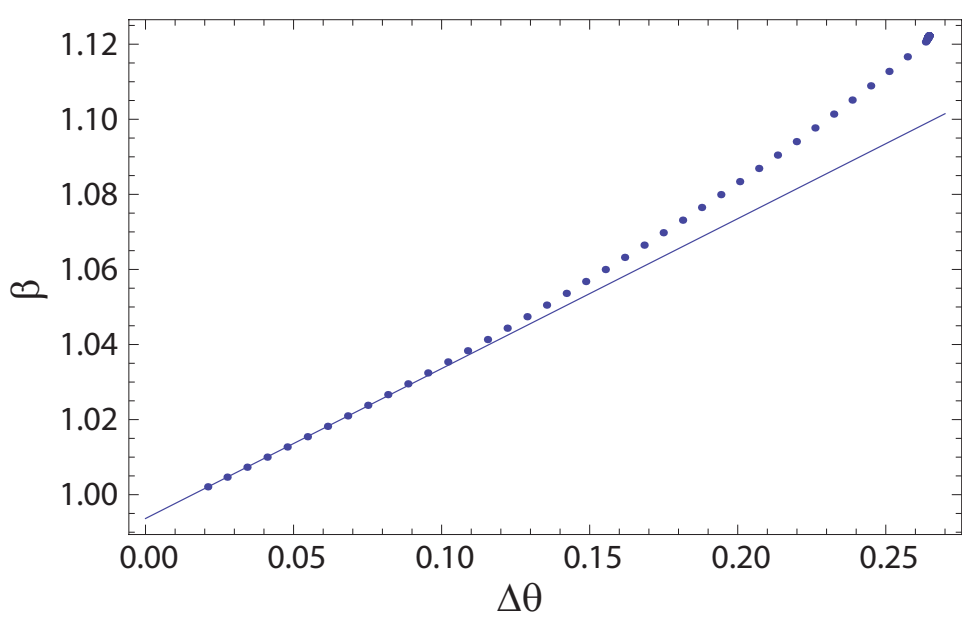

Figura 3.19: Relación de aspecto $\beta$ frente a $\Delta \cos$, para $\theta_{\mathrm{re}}-\theta_{\mathrm{av}}=$ $25^{\circ}$, con $\theta_{\mathrm{av}}=60^{\circ}$. Puntos: resultado de simulación con Surface Evolver. Línea: ajuste por mínimos cuadrados de los datos iniciales.

$\left(\theta_{\mathrm{av}}=70^{\circ}, 110^{\circ}, 130^{\circ}\right)$ o varía muy poco $\left(\theta_{\mathrm{av}}=90^{\circ}\right)$.

\subsubsection{Relación entre el radio medio $R_{\mathrm{a}}$, el semiancho $\omega$ y la relación de aspecto $\beta$}

Se define el radio medio $R_{\mathrm{a}}$ de la base de la gota como aquel que tiene un círculo cuya área sea igual al área de la base de la gota $A$, es decir, $R_{\mathrm{a}}$ viene definido por

$$
A=\pi R_{\mathrm{a}}^{2} .
$$

Teniendo en cuenta que la base de una gota deformada tiene una forma cuya parte superior es aproximadamente semicircular y la parte inferior es aproximadamente semielíptica (véase la figura 3.22), es sencillo obtener una relación entre $R_{\mathrm{a}}$ y el semiancho de la gota $\omega$, como se verá a continuación. Por un lado se tiene que el área de la gota $A$ se puede expresar como

$$
A=\frac{1}{2} A_{\mathrm{c}}+\frac{1}{2} A_{\mathrm{e}}
$$

donde

$$
A_{c}=\pi \cdot \omega^{2}
$$




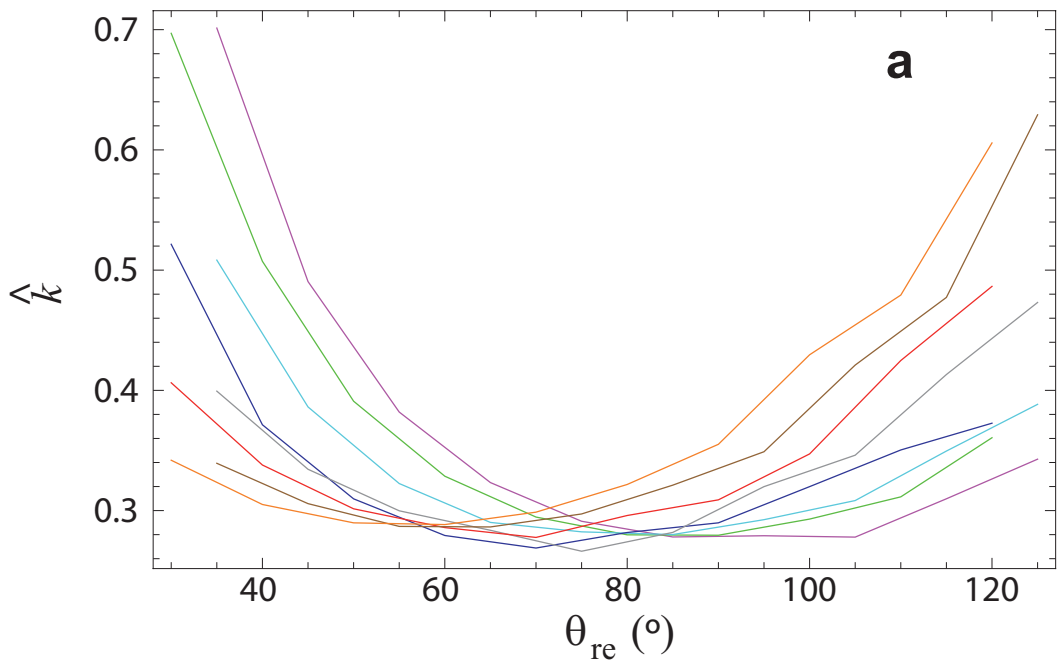

$\Delta \theta=5^{\circ}$

$\Delta \theta=10^{\circ}$

$\Delta \theta=15^{\circ}$

$\Delta \theta=20^{\circ}$

$\Delta \theta=25^{\circ}$

$\Delta \theta=30^{\circ}$

$\Delta \theta=35^{\circ}$

$\Delta \theta=40^{\circ}$

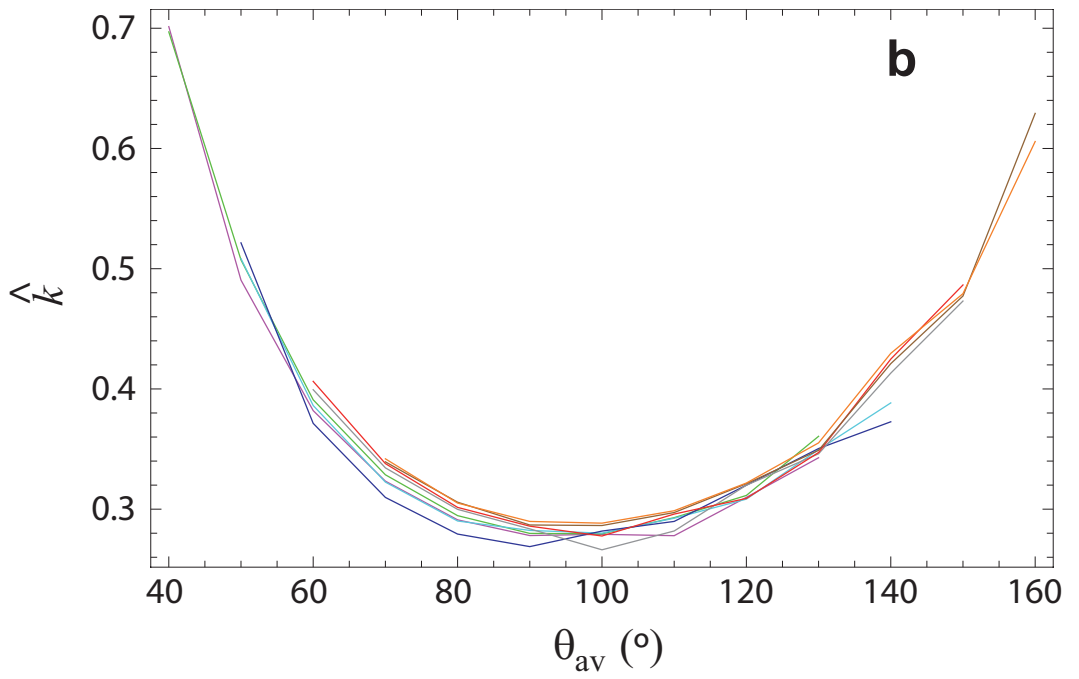

$\Delta \theta=5^{\circ}$

$\Delta \theta=10^{\circ}$

$\Delta \theta=15^{\circ}$

$\Delta \theta=20^{\circ}$

$\Delta \theta=25^{\circ}$

$\Delta \theta=30^{\circ}$

$\Delta \theta=35^{\circ}$

$\Delta \theta=40^{\circ}$

Figura 3.20: Pendiente $\hat{k}$ frente a) al ángulo de contacto de retroceso $\theta_{\mathrm{re}}, y$ b) al ángulo de contacto de avance $\theta_{\mathrm{av}}$. 


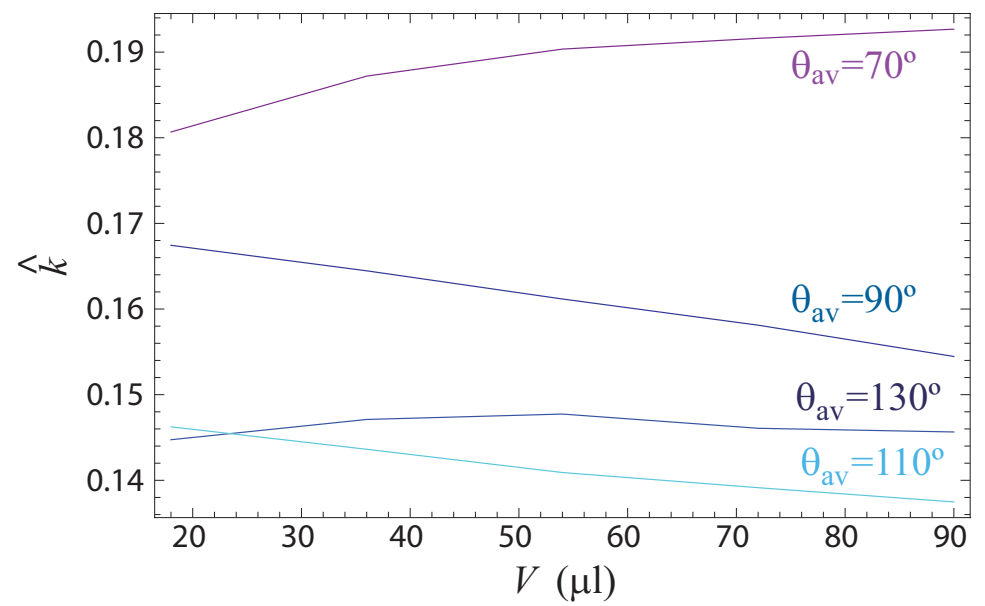

Figura 3.21: Pendiente $\hat{k}$ frente al volumen para una gota de agua. Resultados de simulación con Surface Evolver manteniendo $\Delta \theta=$ $\theta_{\mathrm{av}}-\theta_{\mathrm{re}}=20^{\circ}$ y considerando distintos ángulos de contacto de avance.

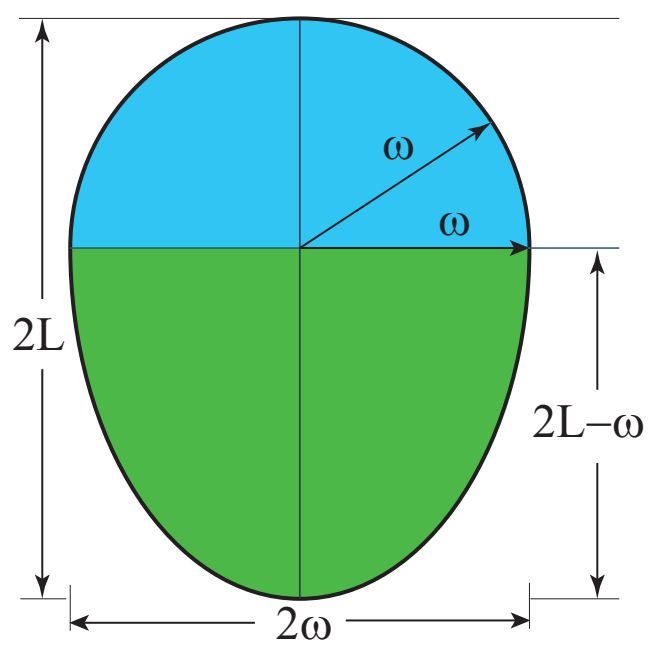

Figura 3.22: Línea de contacto triple de una gota cuando el plano sobre el que se deposita está inclinado un ángulo $0^{\circ}<\alpha<_{c}$, tiene forma aproximadamente semicircular (sombreado azul) en la parte superior y aproximadamente semielíptica (sombreado verde) en la inferior. 


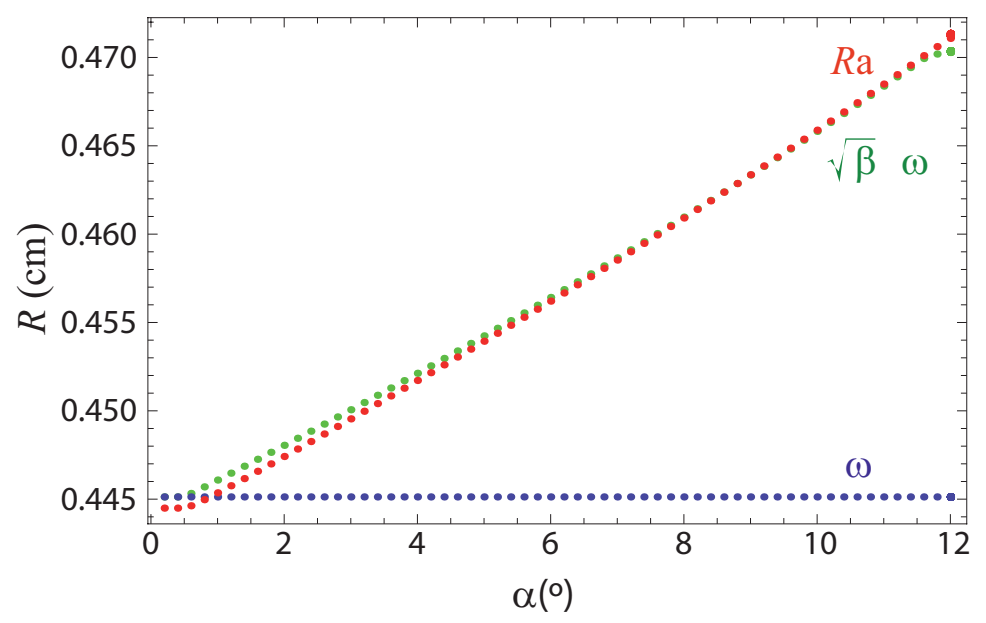

Figura 3.23: Semiancho $\omega$ (puntos azules), radio de la base de la gota obtenido a partir del área de contacto $R_{a}$ (puntos rojos) y producto del semiancho por la raíz cuadrada de la relación de aspecto $\omega \cdot \sqrt{\beta}$ (puntos verdes), frente al al ángulo de inclinación $\alpha$ para una gota de agua de $V=90 \mu \mathrm{l}, \theta_{\min }-\theta_{\max }=20^{\circ} \mathrm{y}$ $\theta_{\mathrm{av}}=70^{\circ}$.

es el área de un círculo de radio igual al semiancho $\omega, \mathrm{y}$

$$
A_{\mathrm{e}}=\pi(2 L-\omega) \cdot \omega,
$$

es el área de una elipse de semiejes $\omega$ y $(2 L-\omega)$.

Sustituyendo (3.16), (3.18) y (3.19) en (3.17) es directo obtener

$$
R_{\mathrm{a}}^{2}=L \omega,
$$

o bien, utilizando la definición (3.14) se llega a una sencilla ecuación que relaciona el radio medio de la línea triple $R_{\mathrm{a}}$ (deducido a partir del área de contacto) con el semiancho de la gota $\omega$, a través de la raíz cuadrada de la relación de aspecto $\beta$

$$
R_{\mathrm{a}}=\sqrt{\beta} \cdot \omega .
$$

Con el objeto de comprobar esta relación, en la figura 3.23 se comparan los resultados de simulación para el semiancho $\omega$, el radio medio $R_{\mathrm{a}}$ y el producto $\sqrt{\beta} \cdot \omega$. 
Estos resultados se representan frente al ángulo de inclinación $\alpha$ para el caso de una gota de agua de volumen $V=90 \mu \mathrm{l}, \theta_{\min }-\theta_{\max }=20^{\circ}$ y $\theta_{\mathrm{av}}=70^{\circ}$. Como se puede comprobar existe un excelente acuerdo entre los resultados obtenidos para $R_{\mathrm{a}}$ y el resultado aproximado $\sqrt{\beta} \cdot \omega$.

Es de destacar que mientras $R_{\mathrm{a}}$ crece (la gota se deforma) el semiancho $\omega$ permanece constante en los resultados de simulación.

\subsubsection{Relación entre la fuerza y el área de la base de la gota}

La expresión (3.21) combinada con los comportamientos lineales de la relación de aspecto [ecuación (3.15)] y de la fuerza de retención (3.9) frente a la diferencia de $\operatorname{cosenos} \Delta \cos =\cos \theta_{\min }-\cos \theta_{\max }$, permite establecer una nueva relación entre el área de la base de la gota y la fuerza de retención, como se verá a continuación. En primer lugar, a partir de (3.15) y de (3.9) se tiene que

$$
\frac{F_{\mathrm{y}}}{\sigma \omega}=\frac{k}{\hat{k}}(\beta-1) \text {. }
$$

Por otro lado, utilizando (3.16) y (3.21), se obtiene

$$
A=\pi R_{\mathrm{a}}^{2}=\pi \beta \omega^{2}
$$

que para el substrato sin inclinar $(\beta=1)$ se reduce a

$$
A_{0}=\pi \omega^{2}
$$

con lo que

$$
\beta=\frac{A}{A_{0}}
$$

y

$$
\frac{F_{\mathrm{y}}}{\sigma \omega}=\frac{k}{\hat{k}}\left(\frac{A}{A_{0}}-1\right)=\frac{k}{\hat{k}} \frac{\Delta A}{A_{0}} .
$$

donde $\Delta A=A-A_{0}$.

La ecuación (3.26) pone de manifiesto la existencia de una relación lineal entre la fuerza $F_{\mathrm{y}} /(\sigma \cdot \omega)$ y el cociente de áreas $A / A_{0}$, como se corrobora en la figura 3.24. En ella se ha considerado una gota de volumen $18 \mu \mathrm{l}$, ángulo de contacto de avance 


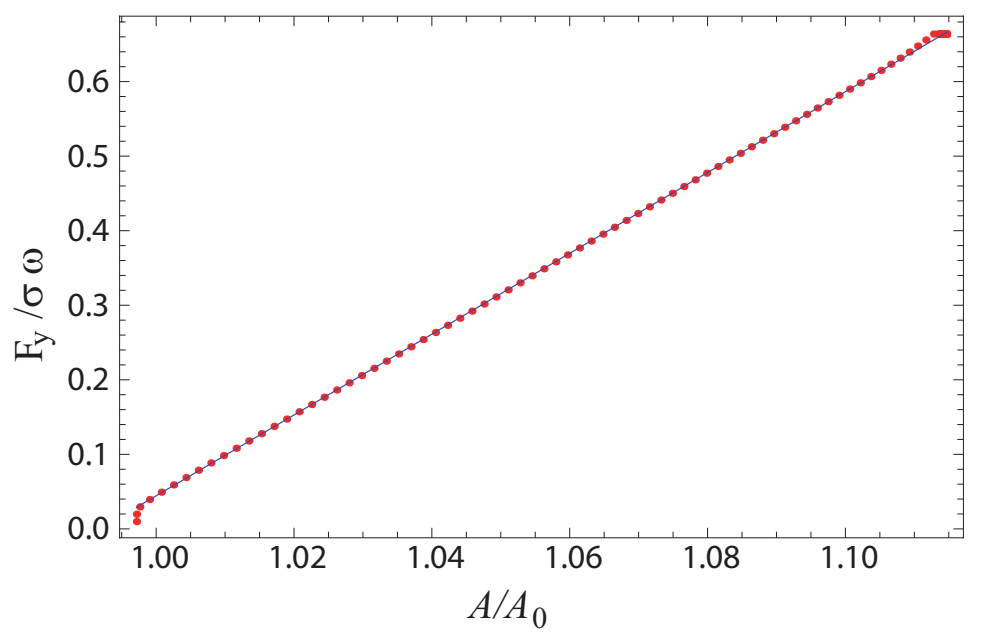

Figura 3.24: Fuerza $F_{\mathrm{y}} / \sigma \omega$ frente al cociente de áreas $A / A_{0}$ (puntos rojos), para una inclinación desde $\alpha=0^{\circ}$ a $\alpha=36^{\circ}$. Resultado de la simulación con Surface Evolver para una gota de agua de $V=18 \mu \mathrm{l}, \theta_{\mathrm{av}}=90^{\circ}$ y $\Delta \theta=\theta_{\mathrm{re}}-\theta_{\mathrm{av}}=20^{\circ}$. La recta azul es un ajuste lineal de los datos.

$\theta_{\mathrm{av}}=70^{\circ}$ y diferencia de ángulos de contacto $\Delta \theta=\theta_{\mathrm{re}}-\theta_{\mathrm{av}}=20^{\circ}$; con una inclinación del substrato sobre el que está depositada dicha gota desde $\alpha=0^{\circ}$ a $\alpha=36^{\circ}$.

Si se fija $\Delta \cos \theta$, y se toman diferentes volúmenes de la gota, las pendientes de las gráficas del tipo de la figura 3.24 son bastante similares. Esto se puede ver en la figura 3.25 en la que se representa la fuerza $F_{\mathrm{y}} /(\sigma \cdot \omega)$ frente al cociente de áreas $A / A_{0}$, a partir de los resultados de las simulaciones con Surface Evolver para una gota de agua con $\theta_{\mathrm{av}}=130^{\circ}, \Delta \theta=\theta_{\mathrm{re}}-\theta_{\mathrm{av}}=20^{\circ} \mathrm{y}$ diferentes volúmenes. Este comportamiento es debido al hecho de que fijado $\theta_{\mathrm{av}}$ y $\Delta \theta$, las pendientes $k$ y $\hat{k}$ varían muy poco con el volumen.

Sin embargo, fijado $V$ y $\Delta \theta$, un cambio de $\theta_{\text {av }}$ da lugar a un cambio en $k / \hat{k}$ con lo que se obtienen pendientes diferentes como se muestra en la figura 3.26 donde se considera una gota de agua de volumen $V=36 \mu \mathrm{l}$, una diferencia entre los ángulos de contacto de avance y retroceso $\Delta \theta=\theta_{\mathrm{av}}-\theta_{\mathrm{re}}=5^{\circ}$, y diversos valores de $\theta_{\mathrm{av}}$. Es claro que las pendientes son diferentes en cada caso, aumentando desde $\theta_{\mathrm{av}}=40^{\circ}$ 


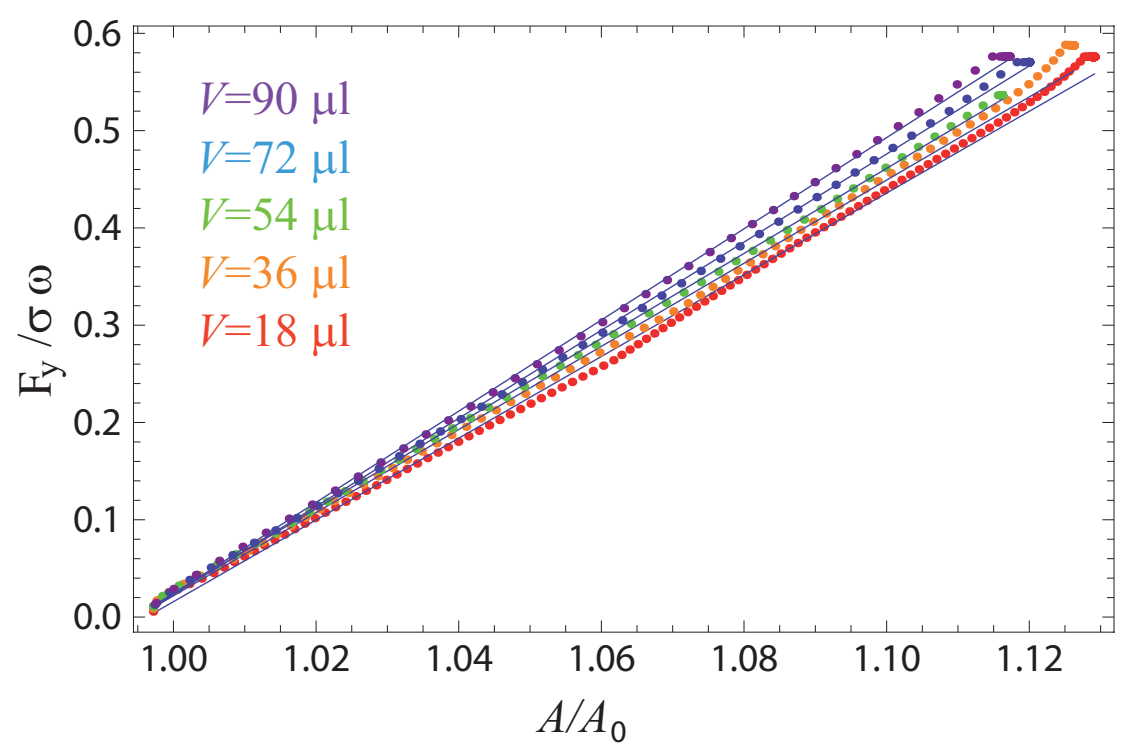

Figura 3.25: Fuerza $F_{\mathrm{y}} / \sigma \omega$ frente al cociente de áreas $A / A_{0}$, para una gota de agua inclinada desde $\alpha=0^{\circ}$. Resultado de la simulación con Surface Evolver para $\theta_{\mathrm{av}}=130^{\circ}, \Delta \theta=\theta_{\mathrm{av}}-\theta_{\mathrm{re}}=20^{\circ}$ y diferentes volúmenes. Las rectas representan ajustes lineales de los datos. 


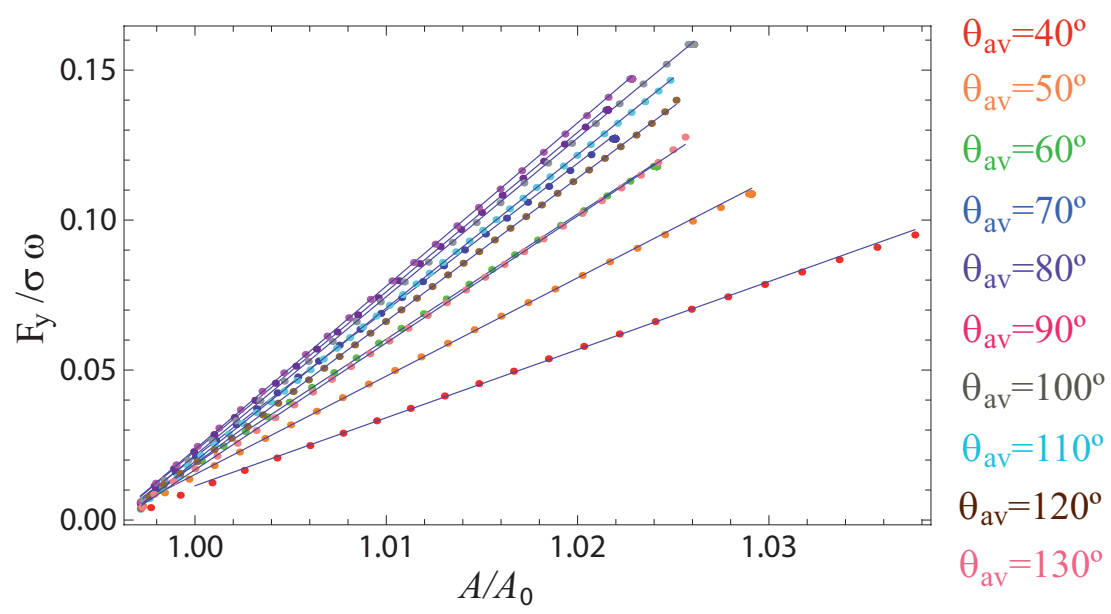

Figura 3.26: Fuerza $F_{y} / \sigma \omega$ frente al cociente de áreas $A / A_{0}$, para una gota de agua inclinada desde $\alpha=0^{\circ}$. Las líneas de puntos son el resultado de la simulación con Surface Evolver para diferentes $\theta_{\mathrm{av}}$ y $\Delta \theta=\theta_{\mathrm{av}}-\theta_{\mathrm{re}}=5^{\circ}$ y $\mathrm{V}=36 \mu \mathrm{l}$. Las rectas representan ajustes lineales de los datos para cada $\theta_{\mathrm{av}}$.

hasta $\theta_{\mathrm{av}}=90^{\circ}$ para, a partir de este caso volver a disminuir.

En la figura 3.27 se representa la variación de las pendientes $k / \hat{k}$ frente a $\theta_{a v}$ para distintos valores de $\Delta \theta$. En todos los casos se obtiene un comportamiento similar con un máximo que se desplaza hacia ángulos de avance mayores a medida que aumenta la diferencia entre el ángulo de avance y el de retroceso $\Delta \theta=\theta_{\mathrm{av}}-\theta_{\mathrm{re}}$.

Finalmente, la figura 3.28 muestra la variación de la pendiente $k / \hat{k}$ con el volumen para gotas de agua con $\Delta \theta=20^{\circ}$, y diferentes valores de $\theta_{\mathrm{av}}$. En todos los casos $k / \hat{k}$ varía muy poco, de acuerdo con los resultados obtenidos para $k$ y para $\hat{k}$ presentados en las figuras 3.13 y 3.21 .

\subsection{Conclusiones}

En este capítulo se ha realizado un estudio de simulación del proceso en el cual una gota de líquido colocada inicialmente sobre una superficie horizontal se inclina gradualmente hasta que desliza por completo. Se ha encontrado que los resultados 


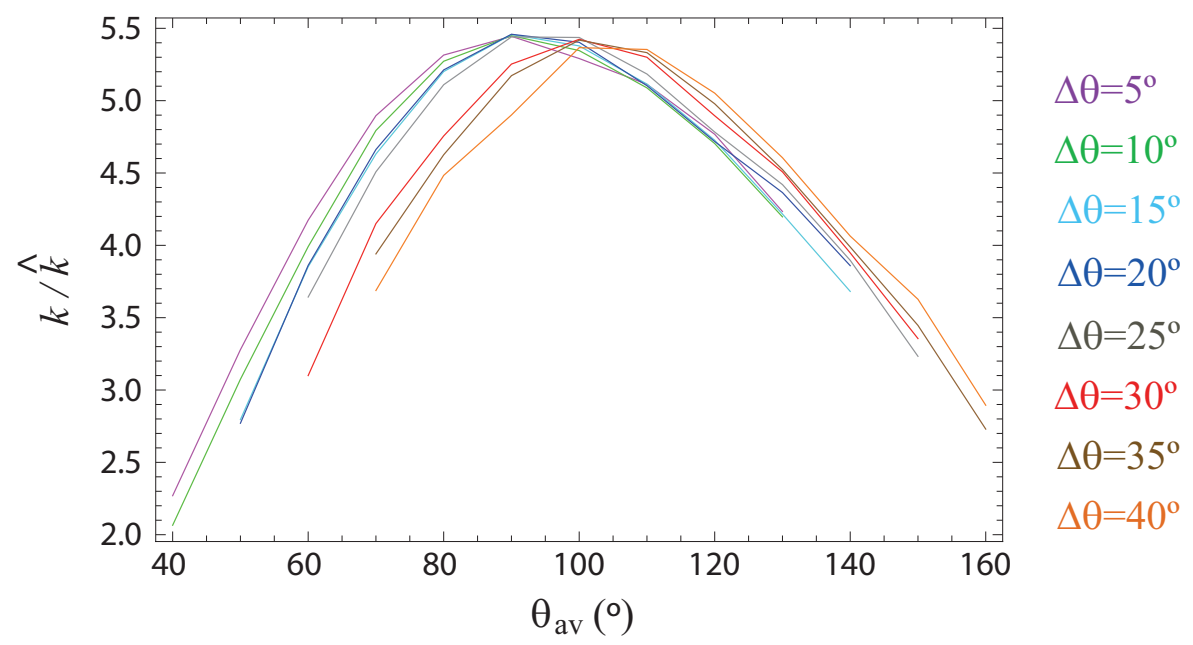

Figura 3.27: Pendiente $k / \hat{k}$ en función del ángulo de contacto de avance $\theta_{\mathrm{av}}$, para diversos $\Delta \theta=\theta_{\mathrm{re}}-\theta_{\mathrm{av}} \operatorname{con} V=36 \mu \mathrm{l}$.

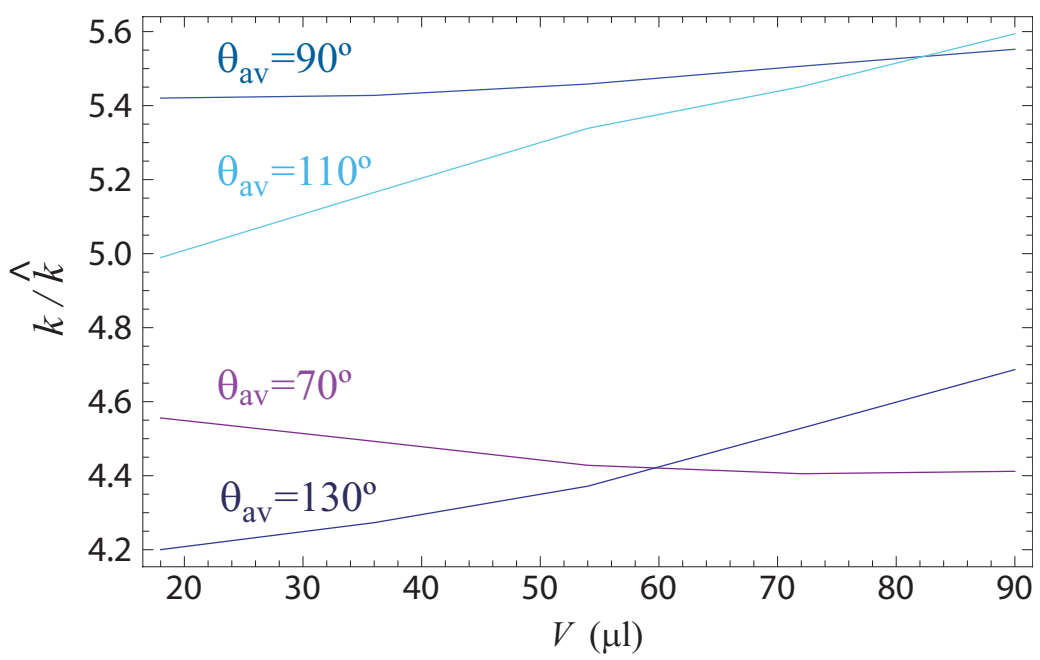

Figura 3.28: Cociente $k / \hat{k}$ respecto al volumen para una gota de agua. Resultados de simulación con Surface Evolver para $\Delta \theta=$ $20^{\circ}$ y diferentes valores de $\theta_{\mathrm{av}}$. 
obtenidos presentan una gran dependencia con los ángulos de contacto inicial de la gota. A continuación se presenta un resumen de los principales resultados para cada condición inicial.

La situación con el ángulo de contacto inicial igual a $\theta_{\text {av }}$ es quizá la más próxima a los experimentos [60]. En este caso el ángulo de contacto máximo $\theta_{\max }$ se mantiene igual a $\theta_{\mathrm{av}}$ mientras que $\theta_{\min }$ disminuye con la inclinación. Bajo estas circunstancias la parte delantera de la línea de contacto triple de la gota se desplaza hacia abajo mientras que la posterior permanece fija. Una vez que $\theta_{\min }$ alcanza $\theta_{\text {re }}$ la parte posterior de la línea de contacto triple se desplaza también pero la gota se mantiene estática. Para la inclinación crítica la mayoría de los ángulos de contacto en la parte frontal son iguales que $\theta_{\mathrm{av}}$, la mayoría de los ángulos de contacto en la parte posterior son iguales a $\theta_{\text {re }}$, y la gota resbala. Para $\theta_{\min }>\theta_{\text {re }}$, la fuerza de retención total adimensional es proporcional a la diferencia de cosenos siguiendo la ecuación (3.9) con una valor de $k$ intermedio entre 2 y $\pi / 2$. Para una gota de agua de volumen $V=0,056 \mathrm{~cm}^{3}$ sobre una superficie inclinada de PCTFE [54] se obtiene un valor de $k=1,779$ pero los resultados para otros sistemas estudiados muestran que $k$ puede tomar otros valores dentro del intervalo $\pi / 2<k<2$ dependiendo del tamaño del sistema y de la histéresis. Para $\theta_{\min }=\theta_{\text {re }}$, la fuerza de retención total adimensional aumenta hasta alcanzar una valor crítico ligeramente inferior que el proporcionado por la ecuación (3.6), es decir, con un valor de $k$ ligeramente menor que $k=2$.

Un ángulo de contacto inicial igual a $\theta_{Y}=\left(\theta_{\mathrm{re}}+\theta_{\mathrm{av}}\right) / 2$ conduce a los resultados previamente obtenidos por métodos de elementos finitos [33]. En este caso $\theta_{\max }$ aumenta y $\theta_{\min }$ disminuye con la inclinación a una velocidad similar mientras que la línea de contacto permanece fija. Una vez que $\theta_{\min }\left(\theta_{\max }\right)$ alcanza $\theta_{\text {re }}\left(\theta_{\mathrm{av}}\right)$ parte de la zona posterior (frontal) se desplaza pero la gota permanece estática. Para el ángulo de inclinación crítico la mayoría de los ángulos de contacto de la parte posterior (frontal) son iguales a $\theta_{\mathrm{re}}\left(\theta_{\mathrm{av}}\right)$, y la gota se desliza por completo. De acuerdo con la ecuación (3.9), para $\theta_{\mathrm{av}}>\theta_{\max }>\theta_{\min }>\theta_{\mathrm{re}}$, la fuerza de retención total adimensional es proporcional a la diferencia de cosenos con una pendiente $k \approx \pi / 2$. Una vez se alcanza la diferencia de cosenos máxima, la fuerza de retención total aumenta hacia un valor crítico caracterizado por un valor de la pendiente $k$ 
ligeramente inferior a 2.

Se encuentran en la literatura resultados experimentales para la evolución de los ángulos de contacto de $\mathrm{Sn}-\mathrm{Ag}$-Cu fundido sobre un substrato inclinado de cobre libre de oxígeno [67]. En este caso el ángulo de contacto inicial es ligeramente menor que $\theta_{\mathrm{av}}$, se trata pues de una situación híbrida por lo que para inclinaciones pequeñas $\cos \theta_{\max }$ se aproxima a $\cos \theta_{\mathrm{av}}$ de un modo lineal mientras que $\cos \theta_{\min }$ aumenta linealmente hacia $\cos \theta_{\mathrm{re}}$. Para inclinaciones mayores, $\theta_{\max }=\theta_{\mathrm{av}} \mathrm{y} \cos \theta_{\min }$ sigue aumentando linealmente hacia $\cos \theta_{\text {re }}$ pero con una pendiente diferente. La comparación de los resultados de simulación con los datos experimentales muestran un ajuste excelente a pesar de ser un sistema con histéresis elevada.

Un análisis de la deformación de la base de la gota en el caso $\theta_{\text {ini }}=\theta_{\text {av }}$ nos ha permitido establecer una serie de relaciones entre la relación de aspecto, el radio medio de la gota, su semiancho, el área de la base y la fuerza de retención. Estas relaciones pueden servir de guía para posteriores estudios experimentales.

Para concluir, en este capítulo se ha visto como el método de simulación basado en Surface Evolver puede reproducir resultados experimentales para gotas de líquido sobre superficies inclinadas donde la histéresis de los ángulos de contacto ocasiona una fuerza de retención capilar que mantiene a la gota estática. La ecuación (3.9) establece una relación lineal entre la fuerza y la diferencia de los cosenos de los ángulos de contacto máximo y mínimo obtenidos en una gota para una inclinación dada. Más aún, la ecuaciones (3.10) y (3.11) muestran que tanto $\cos \theta_{\max }$ como $\cos \theta_{\min }$ se comportan linealmente con el seno del ángulo de inclinación. 



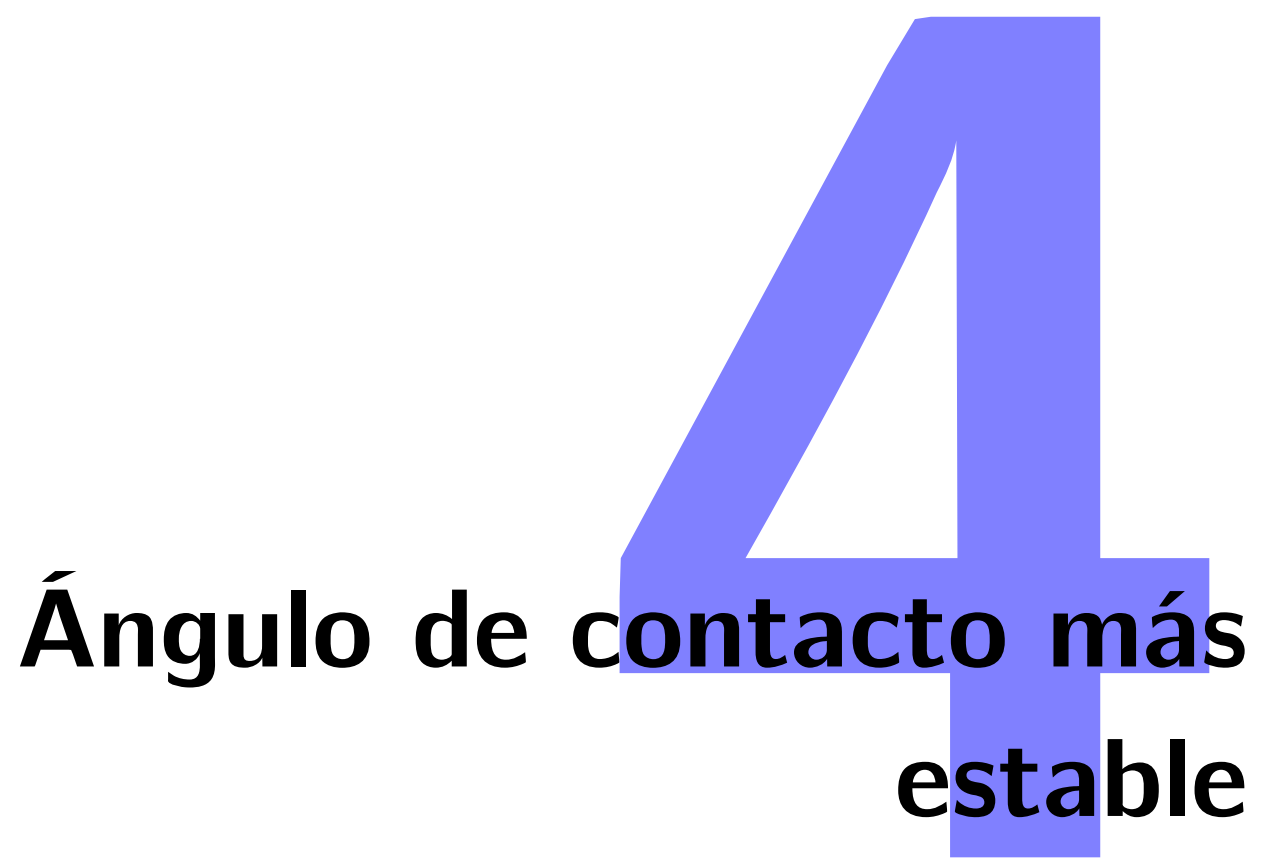

\subsection{Introducción}

La ecuación de Young permite introducir el ángulo de Young como el ángulo de contacto característico de un sistema formado por una gota de líquido sobre una superficie sólida ideal. Desde un punto de vista energético esto quiere decir que la energía del sistema tiene un único mínimo situado en $\theta_{Y}$. Sin embargo, al medir experimentalmente ángulos de contacto, en lugar de obtener un único valor estable, en aplicaciones prácticas se encuentra un intervalo de valores de ángulos de contacto estables. Este hecho se debe a que las superficies reales tienen cierto grado de heterogeneidad química y rugosidad que dan lugar a una energía con múltiples mínimos locales.

Dentro de este intervalo de valores hay tres ángulos de contacto de especial interés: los extremos del intervalo, $\theta_{\mathrm{av}}$ y $\theta_{\text {re }}$ y el ángulo de contacto $\theta_{\text {me }}$ característico del estado más estable del sistema alcanzado en el mínimo global de la energía.

Varios trabajos experimentales $[45,70-74]$ ha empleado la técnica de inducción de vibraciones en el sistema con el objeto de saltar las barreras de potencial existentes entre los estados metaestables y alcanzar el mínimo global [30] y, consecuentemente, 
medir $\theta_{\text {me }}$. Es de destacar que en estos experimentos el sistema debe conservar la simetría cilíndrica tras las vibraciones puesto que la gota en el estado más estable es axisimétrica, como ha sido demostrado teóricamente por Wolansky y Marmur [75]. Muy recientemente Montes et al. [46] han propuesto un método experimental para determinar $\theta_{\text {me }}$ basado en el uso de placas inclinables.

Desde un punto de vista teórico es posible estimar $\theta_{\text {me }}$ a partir de los valores de $\theta_{\text {av }}$ y $\theta_{\text {re }}$ utilizando el promedio de los cosenos

$$
\cos \theta_{\mathrm{me}} \simeq \frac{\cos \theta_{\mathrm{re}}+\cos \theta_{\mathrm{av}}}{2}
$$

como proponen Andrieu et al. a partir de sus medidas experimentales [71], o bien empleando

$$
\theta_{\mathrm{me}} \simeq \frac{\theta_{\mathrm{re}}+\theta_{\mathrm{av}}}{2}
$$

como sugieren los resultados de Montes et al. [74].

En este capítulo se aborda el estudio de $\theta_{\text {me }}$ por medio de simulación empleando estrategias similares a las utilizadas en los trabajos experimentales anteriormente mencionados. En el apartado 4.2 se considera el método de placa inclinable propuesto por Montes et al. [46]. Este método se adapta especialmente bien a un estudio de simulación, como se verá más adelante. En el apartado 4.3 se considera el método de vibraciones mecánicas, inspirado en otros trabajo del mismo grupo $[45,74]$. En este caso las simulaciones con Surface Evolver no permiten hacer una descripción de la dinámica de vibraciones del sistema. Sin embargo, es posible considerar la evolución del mismo a través de sucesivos estados de equilibrio caracterizados por distintos valores de la aceleración de la gravedad en la simulación del sistema. Si se consideran valores oscilantes de $g$ se puede llevar a cabo una pseudodinámica oscilante en el sistema que permitirá estimar el valor del ángulo de contacto más estable.

\subsection{Método de placa inclinable}

Como ya se expuso en detalle en el apartado 3.3, al depositar una gota de líquido sobre una superficie horizontal e inclinarla de forma progresiva, se distinguen tres 
ángulos de inclinación críticos en relación al movimiento de la línea de contacto triple [65]. Estos ángulos se definen como:

- $\alpha_{\mathrm{u}}$ : Ángulo de inclinación crítico para el que se observa el desplazamiento incipiente de la parte posterior (uphill) de la línea de contacto.

- $\alpha_{d}$ : Ángulo de inclinación crítico para el que se observa el desplazamiento incipiente de la parte delantera (downhill) de la línea de contacto.

- $\alpha_{c}$ : Ángulo de inclinación crítico para el que la gota se desplaza por completo.

A partir de estos ángulos Montes et al. [46] introducen dos nuevas definiciones:

- $\alpha_{1}$ : Ángulo de inclinación para el que se observa el primer desplazamiento de la línea de contacto (ya sea la parte delantera o la trasera) durante el proceso de inclinación.

- $\alpha_{2}$ : Ángulo mínimo de inclinación para el que tanto la parte posterior como la delantera de la línea de contacto han iniciado el desplazamiento.

Como se ha visto en el capítulo 3, los valores de estos ángulos críticos dependen fuertemente del ángulo de contacto inicial de la gota, es decir, del ángulo de contacto para inclinación $\alpha=0^{\circ}$. El método experimental recientemente propuesto por Montes et al. [46] considera gotas con distintos ángulos de contacto pertenecientes al intervalo $\theta_{\text {re }}<\theta<\theta_{\text {av }}$ (estos ángulos pueden ser obtenidos añadiendo y/o retirando líquido de la gota). Para cada valor de $\theta$ se inclina gradualmente la placa hasta medir los valores $\alpha_{1}$ y $\alpha_{2}$. De acuerdo con las propuestas de Montes et al. [46] una buena estimación del ángulo de contacto más estable viene dada por el ángulo $\theta$ que permite alcanzar un valor máximo para $\alpha_{1}$. Además en este caso $\alpha_{1}$ y $\alpha_{2}$ son muy próximos y $\alpha_{2}-\alpha_{1}$ alcanza un valor mínimo cercano a cero.

Este método se puede aplicar de manera análoga en simulaciones con Surface Evolver, como se verá a continuación. 


\subsubsection{Resultados}

En el estudio de simulación se han considerado gotas de agua de volumen $V=$ $150 \mu \mathrm{l}$, sobre sustratos formados por siete polímeros distintos: PP, PC, PTFE, PET, UPVC, PDMS y PTFE con dos rugosidades diferentes. Se han elegido estos sistemas para poder comparar con los resultados experimentales de Montes et al. [46].

\section{Determinación de los ángulos de inclinación críticos}

Para cada ángulo inicial se identifican en primer lugar los ángulos de inclinación críticos, $\alpha_{\mathrm{u}}, \alpha_{\mathrm{d}} \mathrm{y} \alpha_{\mathrm{c}}$, realizando la representación gráfica de los ángulos de contacto mínimo y máximo, $\theta_{\min }, \theta_{\max }$, frente al ángulo de inclinación del plano, $\alpha$.

En la figura 4.1 se muestran los resultados obtenidos a través de simulaciones de una gota de agua de $150 \mu \mathrm{l}$ sobre PTFEr, para diferentes valores del ángulo de contacto inicial $\theta_{0}$ comprendidos entre el ángulo de contacto de retroceso y el de avance, $\theta_{\mathrm{re}} \leq \theta_{0} \leq \theta_{\mathrm{av}}$. Para este sistema se toma $\theta_{\mathrm{re}}=87,9^{\circ}$ y $\theta_{\mathrm{av}}=126^{\circ}$ [46]. Los cuadrados se corresponden con los valores de los ángulos de contacto máximos, pertenecientes a la zona delantera de la línea de contacto triple (avance) y, los círculos con los valores de los ángulos de contacto mínimos, pertenecientes a la zona posterior de la línea de contacto triple (retroceso). El comportamiento se comprende mejor examinando en detalle algunas de estas situaciones:

- Ángulo de contacto inicial $\theta_{0}=\theta_{\text {re }}=87,90^{\circ}$. En este caso, nada más inclinar la gota se observa un movimiento incipiente de la parte posterior mientras que la parte delantera permanece fija, esto es, $\alpha_{\mathrm{u}}=0^{\circ}$. Para $\alpha_{\mathrm{d}}=14,00^{\circ}$, el ángulo de contacto máximo de la parte delantera alcanza el valor $\theta_{\mathrm{av}}=126^{\circ}$ y se observa el desplazamiento incipiente de dicha zona mientras que la parte posterior también se desplaza aunque la gota permanece fija. Por tanto, en este caso $\alpha_{1}=\alpha_{\mathrm{u}}$ y $\alpha_{2}=\alpha_{\mathrm{d}}$. La gota se desliza por completo a partir de $\alpha_{\mathrm{c}}=17,30^{\circ}$.

- Ángulo de contacto inicial $\theta_{0}=106,95^{\circ}$. Desde $\alpha=0^{\circ}$ hasta $\alpha_{\mathrm{d}}=11,50^{\circ}$ la línea de contacto tiene una forma circular y permanece fija. Para $\alpha_{d}=11,50^{\circ}$, 

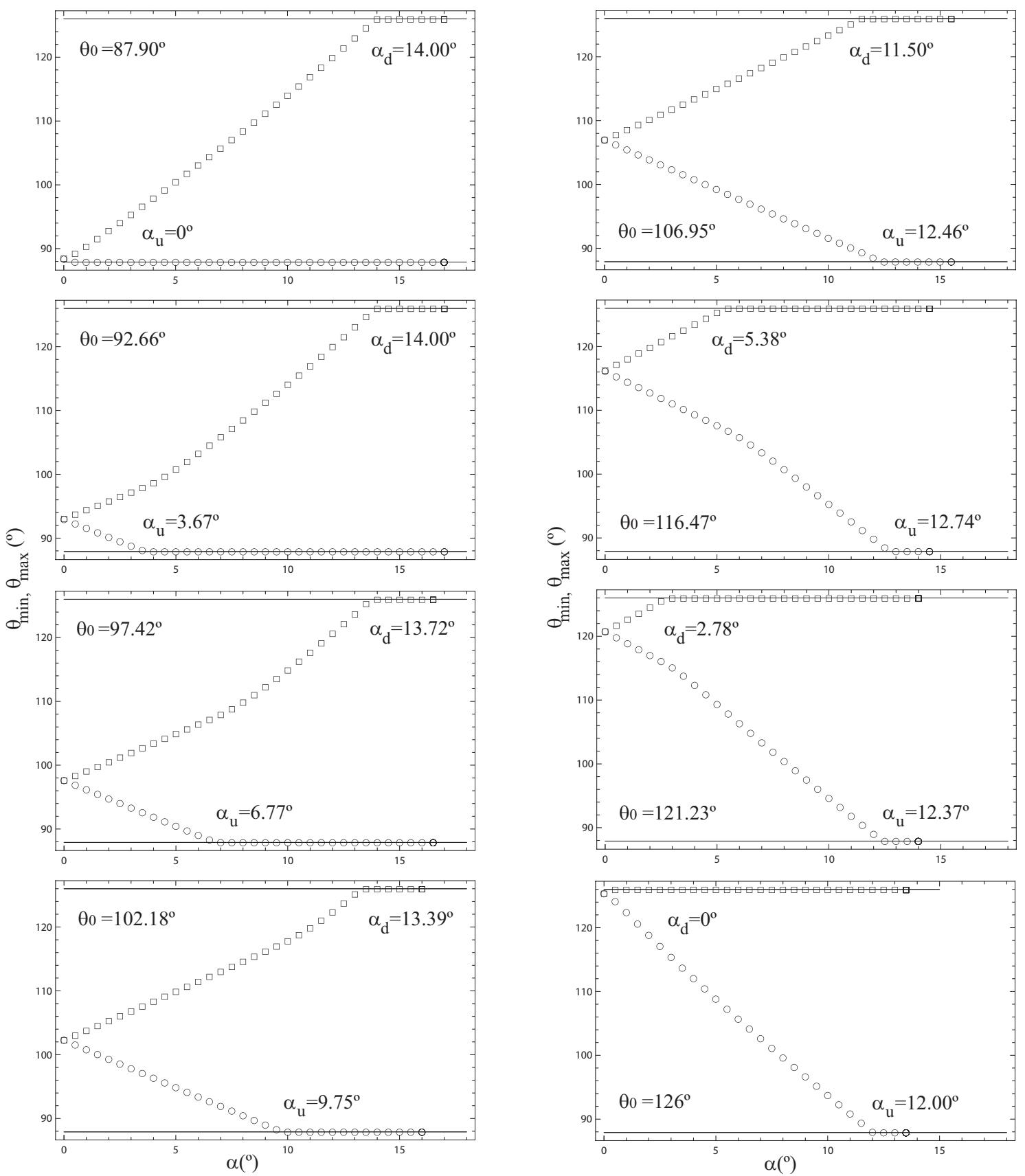

Figura 4.1: Ángulos de contacto mínimo (círculos) y máximo (cuadrados) frente a la inclinación del substrato, para diferentes valores del ángulo de contacto inicial, en una simulación de placa inclinable para una gota de agua de $V=150 \mu /$ sobre PTFEr con $\theta_{\text {re }}=87,9^{\circ}$ y $\theta_{\mathrm{av}}=126^{\circ}$. 
el ángulo de contacto máximo de la parte frontal alcanza el valor $\theta_{\mathrm{av}}=126^{\circ} \mathrm{y}$ se observa el desplazamiento de una parte del frente de la gota mientras que la parte posterior permanece fija. Para $\alpha_{\mathrm{u}}=12,46^{\circ}$, el ángulo de contacto mínimo de la zona posterior alcanza el valor $\theta_{\text {re }}=87,90^{\circ}$ y se observa el desplazamiento de parte de la zona posterior. En este caso $\alpha_{1}=\alpha_{\mathrm{d}}$ y $\alpha_{2}=\alpha_{\mathrm{u}}$. La gota se desliza por completo a partir de $\alpha_{\mathrm{c}}=15,25^{\circ}$.

- Ángulo de contacto inicial $\theta_{0}=\theta_{\mathrm{av}}=126^{\circ}$. Al inclinar la gota se observa el desplazamiento incipiente del frente de la gota mientras que la parte posterior permanece fija, en este caso $\alpha_{\mathrm{d}}=0^{\circ}$. Para $\alpha_{\mathrm{u}}=12,00^{\circ}$, el ángulo de contacto mínimo de la parte posterior alcanza el valor $\theta_{\mathrm{re}}=87,9^{\circ}$ y se observa el desplazamiento de parte de dicha zona mientras que el frente de la gota permanece fija. En este caso $\alpha_{1}=\alpha_{\mathrm{d}}$ y $\alpha_{2}=\alpha_{\mathrm{u}}$. La gota se desliza por completo a partir de $\alpha_{\mathrm{c}}=13,44^{\circ}$.

- En el resto de los casos se dan situaciones intermedias entre las tres mencionadas. En todas ellas se pone de manifiesto un cambio de la pendiente del ángulo de contacto máximo o mínimo cuando se alcanza el ángulo crítico $\alpha_{\mathrm{u}}$ o $\alpha_{\mathrm{d}}$, respectivamente. Por ejemplo, para $\theta_{0}=92,66^{\circ}$ y $\alpha_{\mathrm{u}}=3,67^{\circ}$, la zona posterior alcanza el valor mínimo del ángulo de contacto $\theta_{\mathrm{re}}=87,9^{\circ}$ y en ese instante cambia la pendiente de $\theta_{\max }$ frente a $\alpha$. De modo análogo se comportan aquellas gotas con ángulos de contacto inicial cercanos al ángulo de avance.

En la figura 4.2a se representa el ángulo de inclinación crítico $\alpha_{1}$ obtenido mediante simulación con Surface Evolver, frente al ángulo de contacto inicial $\theta_{0}$, para una gota de agua de $150 \mu \mathrm{l}$ sobre PTFEr, cuando esta superficie se inclina progresivamente desde la horizontal. En esta gráfica se observa que $\alpha_{1}$ alcanza un valor máximo en torno a $\theta_{0} \approx 106^{\circ}$. La figura $4.2 \mathrm{~b}$ muestra el ángulo de inclinación crítico $\alpha_{2}$ frente al ángulo de contacto inicial $\theta_{0}$. Como se puede comprobar $\alpha_{2}$ varía muy poco. Por otra parte, cuando $\alpha_{1}$ alcanza su valor máximo toma un valor próximo a $\alpha_{2}$, es decir $\alpha_{1}-\alpha_{2}$ es cercano a cero. Este comportamiento se ha verificado para el resto de superficies de polímeros estudiadas como se puede ver en la figura 4.3 

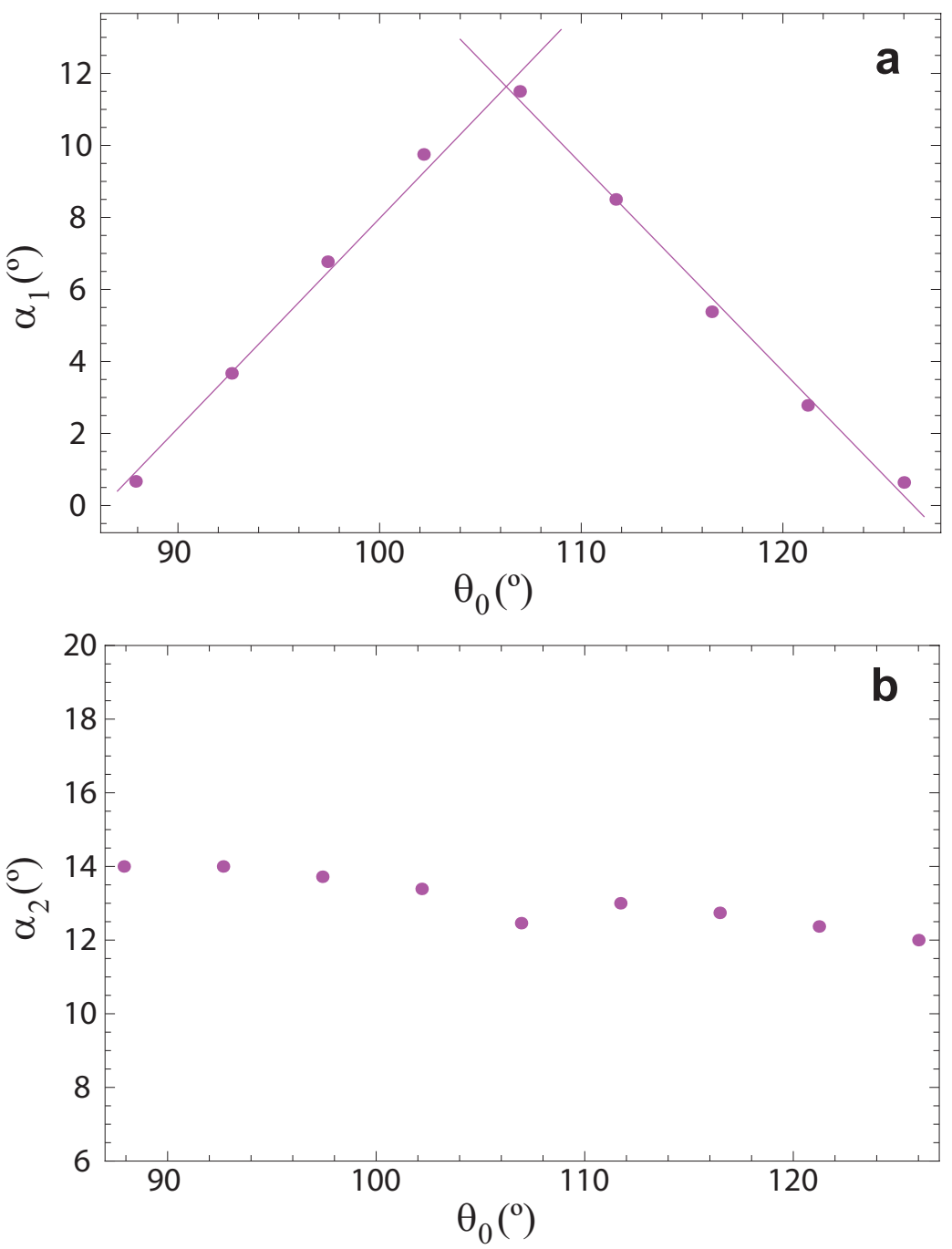

Figura 4.2: Ángulos de inclinación críticos $\alpha_{1}$ y $\alpha_{2}$ frente al ángulo de contacto inicial. Simulación de placa inclinable para una gota de agua de $V=150 \mu /$ sobre PTFEr. Las rectas representan el ajuste por mínimos cuadrados de los datos. 

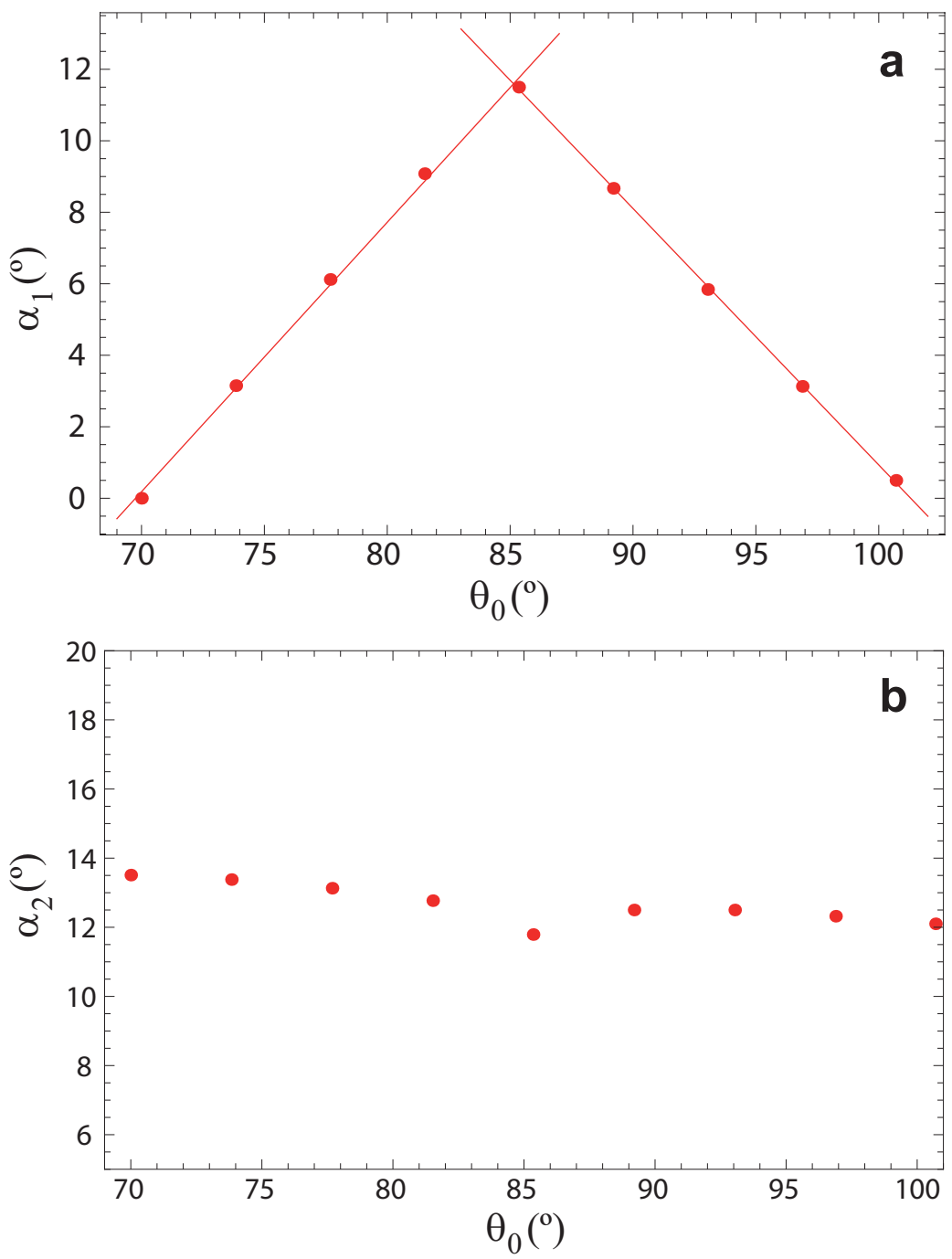

Figura 4.3: Ángulos de inclinación críticos $\alpha_{1}$ y $\alpha_{2}$ frente al ángulo de contacto inicial. Simulación de placa inclinable para una gota de agua de $V=150 \mu /$ sobre $P P$. Las rectas representan el ajuste por mínimos cuadrados de los datos. 


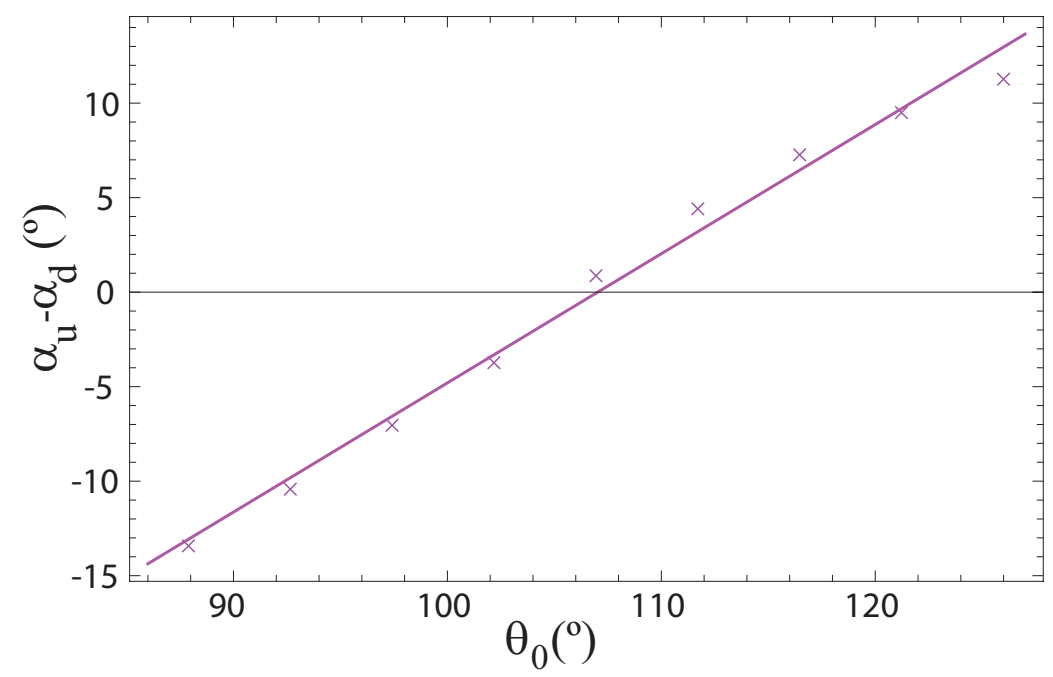

Figura 4.4: Diferencia entre el ángulo de inclinación crítico de la parte posterior $\alpha_{\mathrm{u}}$ y frontal $\alpha_{\mathrm{d}}$, frente al ángulo de contacto inicial. Simulación de placa inclinable para una gota de agua de $V=150 \mu /$ sobre PTFEr. La recta es un ajuste lineal de los datos.

para el caso de gotas de agua de $150 \mu \mathrm{l}$ sobre PP (en este caso $\theta_{0} \approx 85^{\circ}$ ), y está de acuerdo con los resultados experimentales de Montes et al. [46].

\section{Determinación del ángulo de contacto más estable}

Los resultados presentados en las figuras 4.2 a y 4 .3a permiten obtener el ángulo de contacto más estable a partir del valor de $\theta_{0}$ que da lugar a un máximo para $\alpha_{1}$. Una manera sencilla de obtener esta máximo consiste en realizar ajustes lineales por mínimos cuadrados para los datos crecientes y otro para los decrecientes. La intersección de estas dos rectas de regresión permite determinar el ángulo de contacto más estable, como se muestra en $4.2 \mathrm{a}$ y $4.3 \mathrm{a}$. El ángulo de contacto más estable obtenido así se denota $\theta_{\text {tilt }}^{A}$.

El ángulo de contacto más estable también se puede determinar realizando la representación gráfica de la diferencia entre los ángulos críticos, $\alpha_{u}-\alpha_{d}$, frente al ángulo de contacto inicial $\theta_{0}$, y calculando el mínimo valor absoluto de dicha diferencia, como se muestra en la figura 4.4 para el caso de una gota de de agua de volumen 


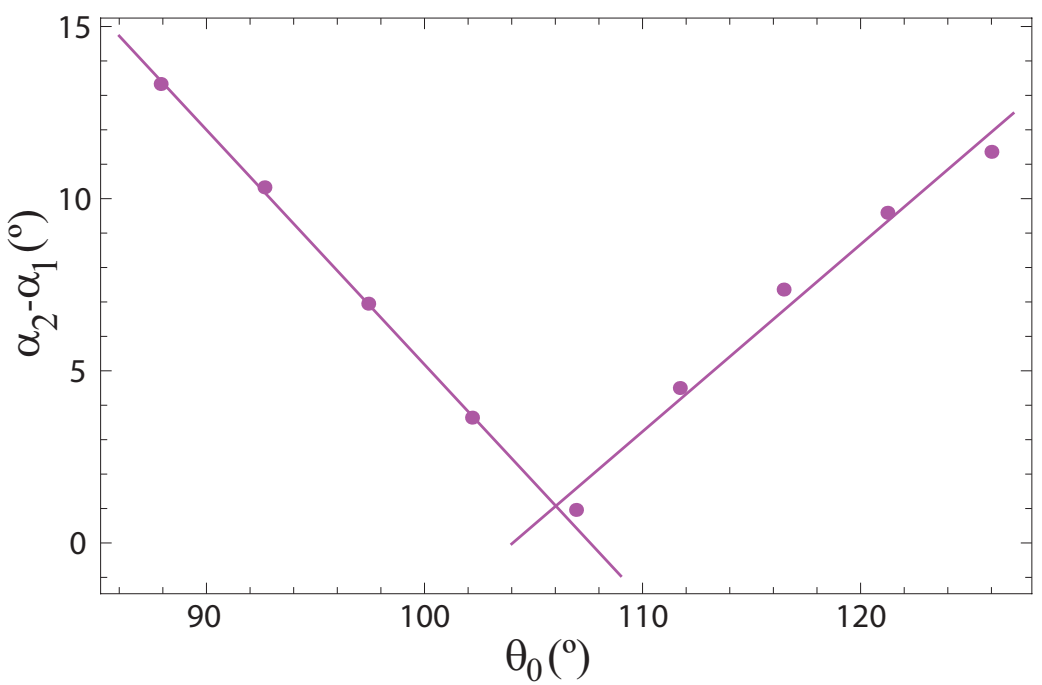

Figura 4.5: Diferencia entre los ángulos de inclinación críticos máximo y mínino, frente al ángulo de contacto inicial. Simulación de placa inclinable para una gota de agua de $V=150 \mu /$ sobre PTFEr. Las rectas son ajustes lineales de los datos.

$150 \mu \mathrm{l}$ sobre PTFEr. En ella se recogen las simulaciones para los ángulos de contacto iniciales $\theta_{0}=87,9^{\circ}, 92,66^{\circ}, 97,42^{\circ}, 102,18^{\circ}, 106,95^{\circ}, 111,71^{\circ}, 116,47^{\circ}, 121,23^{\circ} \mathrm{y}$ $126^{\circ}$. Una vez realizado un ajuste lineal por mínimos cuadrados de los datos se calcula la intersección de dicha recta con el eje $x$, es decir $\alpha_{\mathrm{u}}-\alpha_{\mathrm{d}}=0$, obteniendo un valor de $107,025^{\circ}$ en este ejemplo concreto. El ángulo de contacto más estable obtenido así se denota $\theta_{\text {tilt }}^{B}$. En el cuadro 4.1 se exponen los resultados obtenidos para el ángulo de contacto mas estable para éste y el resto de polímeros estudiados, mediante los métodos aquí presentados.

La figura 4.5 muestra la diferencia entre los ángulos de inclinación críticos máximo y mínino $\alpha_{2}-\alpha_{1}$, frente al ángulo de contacto inicial $\theta_{0}$, procedentes de una simulación de placa inclinable para una gota de agua de $V=150 \mu$ l sobre PTFEr. Las rectas representan ajustes por mínimos cuadrados de los datos. Claramente existe un mínimo para un ángulo de contacto cuya diferencia $\alpha_{2}-\alpha_{1} \rightarrow 0$, y este valor debe coincidir (salvo errores numéricos) con el ángulo de contacto más estable obtenido en la figura 4.4 ya que la representación es análoga a la de esa figura, debido a la 
Método de placa inclinable: ángulo de contacto más estable

\begin{tabular}{ccccccc}
\hline Polímero & $\theta_{\mathrm{av}}\left(^{\circ}\right)$ & $\theta_{\mathrm{re}}\left(^{\circ}\right)$ & $\left\langle\theta>\left(^{\circ}\right)\right.$ & $\left\langle\theta_{\mathrm{cos}}>\left(^{\circ}\right)\right.$ & $\theta_{\mathrm{tilt}}^{\mathrm{A}}\left({ }^{\circ}\right)$ & $\theta_{\mathrm{tilt}}^{\mathrm{B}}\left({ }^{\circ}\right)$ \\
\hline PP & $100,7 \pm 1,6$ & $70 \pm 3$ & 85,35 & 85,51 & 85,14 & 85,74 \\
PC & $86,2 \pm 1,5$ & $48 \pm 2$ & 67,1 & 68,42 & 67,11 & 67,86 \\
PET & $80 \pm 2$ & $49,7 \pm 1,5$ & 64,85 & 65,78 & 64,87 & 65,33 \\
uPVC & $86 \pm 2$ & $50 \pm 2$ & 68 & 69,13 & 68,03 & 68,70 \\
PTFEs & $110 \pm 4$ & $95,6 \pm 1,0$ & 102,8 & 102,70 & 102,74 & 102,85 \\
PTFEr & $126 \pm 2$ & $87,9 \pm 1,3$ & 106,95 & 105,99 & 106,28 & 107,02 \\
PDMSs & $120,2 \pm 1,9$ & $65,2 \pm 1,9$ & 92,7 & 92,39 & 91,26 & 93,31 \\
\hline
\end{tabular}

Cuadro 4.1: Ángulos de contacto de avance y retroceso [46], ángulo de contacto más estable obtenido a partir de la media aritmética de estos valores (4.1) así como el valor obtenido de la media de los cosenos (4.2), y los resultados obtenidos mediante simulación para el método de placa inclinable utilizando los procedimientos $A$ y $B$. Se considera una gota de agua de $150 \mu$ l sobre varios polímeros. 
propia definición de los ángulos de inclinación críticos $\alpha_{u}, \alpha_{d}, \alpha_{1}$ y $\alpha_{2}$.

\section{Dependencia del ángulo de contacto más estable con el volumen}

A continuación se va a analizar la dependencia con el volumen de los valores del ángulo de contacto más estable obtenidos con la simulación del método de placa inclinable. Para ello se realizan simulaciones con Surface Evolver de gotas de agua sobre superficies de uPVC repitiendo experimentos similares a los de las figuras 4.2, 4.3 y 4.4 , pero con volúmenes distintos. Los resultados de la simulación se recogen en la figura 4.6 para los ángulos de contacto iniciales $\theta_{0}=50,0^{\circ}, 53,6^{\circ}, 57,2^{\circ}, 60,8^{\circ}$, $64,4^{\circ}, 68,0^{\circ}, 71,6^{\circ}, 75,2^{\circ}, 78,8^{\circ}, 82,4^{\circ}$ y $86,0^{\circ}$. En la figura 4.6 a se representa el ángulo de inclinación mínimo $\alpha_{1}$ frente al ángulo de contacto inicial $\theta_{0}$ para gotas de volumen $100 \mu \mathrm{l}$ (cuadrados verdes), $120 \mu \mathrm{l}$ (círculos rojos) y $150 \mu \mathrm{l}$ (triángulos azules) sobre uPVC. Se observa que el máximo, que corresponde al ángulo de contacto más estable, se alcanza en valores similares del ángulo de contacto inicial $\theta_{0}$ para los diferentes volúmenes examinados.

En la figura 4.6b se recogen las diferencias entre los ángulos de inclinación críticos de la parte posterior y frontal de la zona de contacto $\alpha_{\mathrm{u}}-\alpha_{\mathrm{d}}$, frente al ángulo de contacto inicial $\theta_{0}$, para el mismo sistema y volúmenes de la figura 4.6a. Las rectas se corresponden con ajustes lineales de los datos correspondientes a cada volumen. Como se ha indicado previamente, la intersección de cada línea con el eje de abscisas en la figura $4.6 \mathrm{~b}$ se corresponde con el ángulo de contacto más estable, y como sucede en el caso anterior, los resultados obtenidos son similares. Esto indica que el valor del ángulo de contacto más estable de un sistema agua-uPVC es independiente del volumen de la gota dentro del intervalo [100 $\mu \mathrm{l}-150 \mu \mathrm{l}]$, como demuestran experimentalmente Montes et al. [46] en un trabajo reciente.

Se recogen en el cuadro 4.2 los valores numéricos del ángulo de contacto más estable obtenidos mediante simulación con Surface Evolver para gotas de agua de diferente volumen sobre UPVC, para los dos métodos de la figura 4.6:

Método A: calculando el máximo de la representación gráfica del ángulo de inclinación crítico mínino $\alpha_{1}$ frente al ángulo de contacto inicial $\theta_{0}$ (figura 4.6a); 

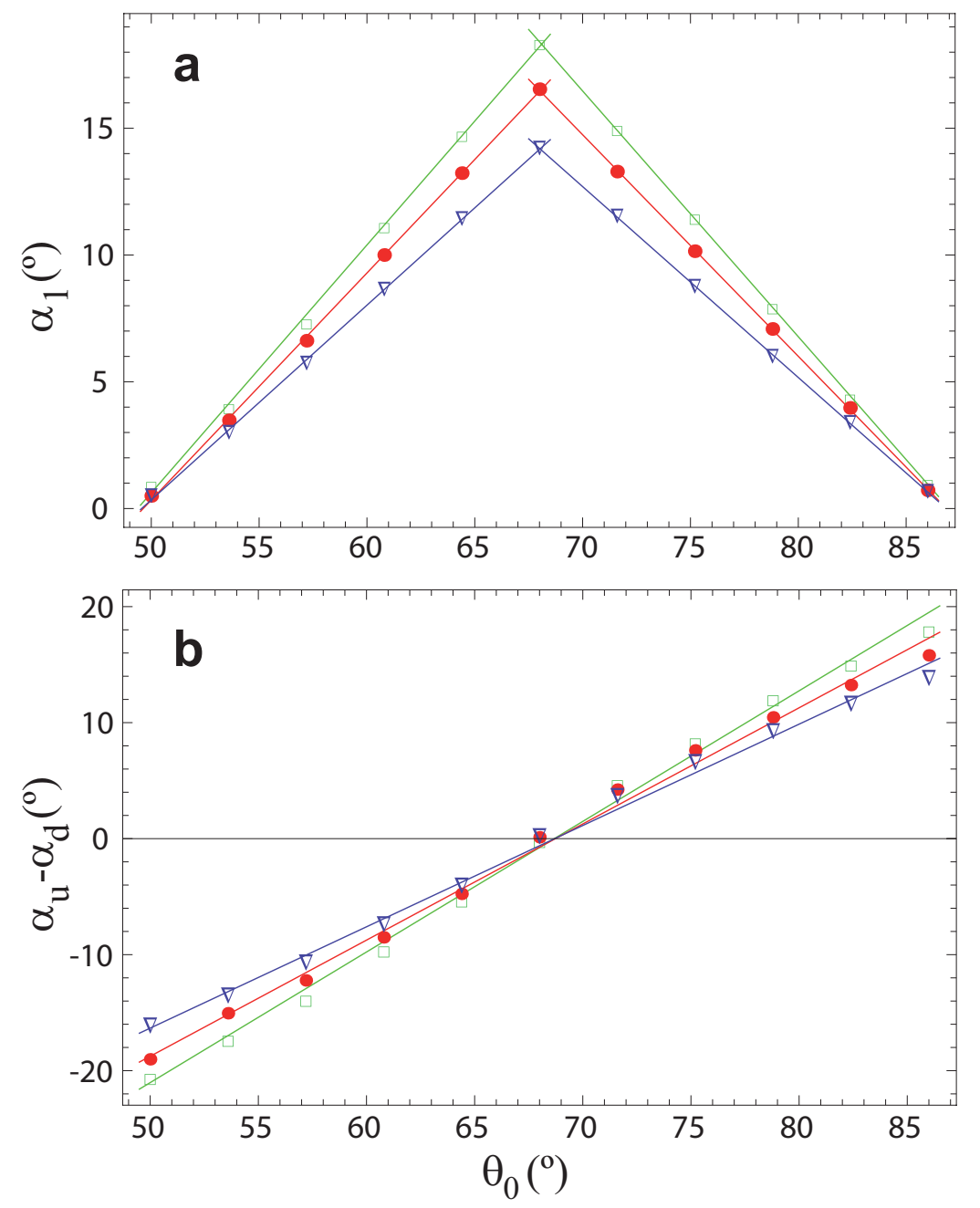

Figura 4.6: a) Ángulo de inclinación crítico mínino $\alpha_{1}$ y b) diferencia de ángulos de inclinación críticos de la parte posterior y frontal de la zona de contacto $\alpha_{\mathrm{u}}-\alpha_{\mathrm{d}}$, frente al ángulo de contacto inicial $\theta_{0}$ para simulaciones de una gota de agua de volumen $100 \mu \mathrm{l}$ (cuadrados verdes), $120 \mu /$ (círculos rojos) y $150 \mu l$ (triángulos azules) sobre uPVC. Las rectas representan ajustes lineales de los datos. El máximo de los valores en a) y la intersección de cada línea con el eje de abscisas en b) debe corresponder al ángulo de contacto más estable. 


\begin{tabular}{ccc}
\hline Volumen $(\mu \mathrm{l})$ & $\theta_{\text {tilt }}^{\mathrm{A}}\left({ }^{\circ}\right)$ & $\theta_{\text {tilt }}^{\mathrm{B}}\left({ }^{\circ}\right)$ \\
\hline 100 & 68,01 & 68,68 \\
120 & 68,02 & 68,74 \\
150 & 68,03 & 68,70 \\
\hline
\end{tabular}

Cuadro 4.2: Valores del ángulo de contacto más estable para gotas de agua de diferentes volúmenes sobre uPVC; obtenidos por el método $A\left(\theta_{\text {tilt }}^{\mathrm{A}}\right)$ y por el método $B\left(\theta_{\mathrm{tilt}}^{\mathrm{B}}\right)$.

Método B: hallando el punto de corte con el eje de abscisas de la representación gráfica de la diferencia de ángulos de inclinación críticos de la parte posterior y frontal de la zona de contacto $\alpha_{\mathrm{u}}-\alpha_{\mathrm{d}}$, frente al ángulo de contacto inicial $\theta_{0}$ (figura 4.6b).

Los datos presentados en el cuadro 4.2 ponen de manifiesto por un lado que para un mismo método los resultados apenas cambian con el volumen y por otro que la estimación del ángulo de contacto más estable dada por el método $A, \theta_{\text {tilt }}^{B}$, sobreestima ligeramente los dados por el método B, $\theta_{\text {tilt }}^{A}$. Este hecho es debido a que los datos presentados en la figura $4.6 \mathrm{~b}$, no tiene un comportamiento totalmente lineal y aparecen desviaciones. Estas desviaciones en el caso de la figura 4.6b sobreestiman el valor del ángulo de contacto más estable pues como se puede ver, la intersección de las rectas de ajuste con el eje de abscisas ocurre para valores de $\theta_{0}$ ligeramente mayores a los resultados de simulación.

\subsection{Método de vibraciones mecánicas}

Desde un punto de vista experimental, el método de las vibraciones mecánicas consiste en someter a una gota sésil sobre un sustrato horizontal a vibraciones verticales. Estas vibraciones pueden evitar que el sistema quede atrapado en estados metaestables, permitiendo que se alcance el estado de mínima energía caracterizado por el ángulo de contacto más estable, que puede ser medido a continuación [71]. Recientemente Rodríguez-Valverde et al. [45] han propuesto una mejora de este 
método consistente en preparar el sistema en diferentes estados metaestables para un determinado volumen. Cada uno de estos estados metaestables viene determinado por un ángulo de contacto inicial $\theta_{0}$ perteneciente al intervalo $\theta_{\text {re }}<\theta_{0}<\theta_{\text {av }}$ y es sometido a vibración tras la que alcanza un estado final con ángulo de contacto $\theta_{\text {vib }}$ que en principio no depende de la historia previa del sistema. El ángulo $\theta_{0}$ para el cual la diferencia $\theta_{\text {vib }}-\theta_{0}$ es mínima (próxima a cero) es el ángulo de contacto más estable.

En principio, no se puede realizar la simulación de este método experimental por medio de Surface Evolver pues este programa sólo permite obtener resultados en el equilibrio y esto impide simular el proceso de vibración de las gotas. Sin embargo, cuando un sistema está sometido a vibraciones armónicas, las aceleraciones también siguen una función armónica de igual frecuencia y fase. Se puede entonces simular un proceso en el cual se va variando el valor de la aceleración de la gravedad $g$ con respecto al valor del sistema en reposo $g_{0} \simeq 9,8 \mathrm{~m} / \mathrm{s}^{2}$. Para cada valor de $g$ se obtiene una configuración estable diferente cuyo ángulo de contacto dependerá de la histéresis y del estado previo del sistema. Aunque este esquema de simulación no reproduce la dinámica de una gota que vibra, tiene las mismas consecuencias prácticas pues permite que el sistema modifique su ángulo de contacto. Si la variación de $g$ tiene suficiente amplitud, el ángulo de contacto puede alcanzar los valores $\theta_{\text {re }}$ y $\theta_{\text {av }}$ con lo que la línea triple sufrirá un desplazamiento tal que el radio del círculo de contacto disminuirá para $\theta_{\mathrm{re}} \mathrm{O}$ aumentará para $\theta_{\mathrm{av}}$.

Un ejemplo de la función oscilante que se utiliza para variar el cociente $g / g_{0}$ viene dado por la figura 4.7a. En esta figura se puede observar como partiendo de un valor de $g / g_{0}=1$ se va incrementando la amplitud de las oscilaciones hasta un determinado valor, posteriormente se disminuye muy lentamente la amplitud y finalmente se disminuye más rápidamente hasta alcanzar de nuevo el valor inicial $g / g_{0}=1$. La envolvente considerada pretende reproducir el comportamiento experimental donde siempre hay un transitorio inicial hasta que se alcanza la amplitud máxima de oscilación y un periodo de amortiguamiento final hasta alcanzar el equilibrio.

La amplitud máxima de oscilación se elige de manera que el sistema pueda alcanzar tanto el ángulo de avance como el de retroceso, como se muestra en la figura 

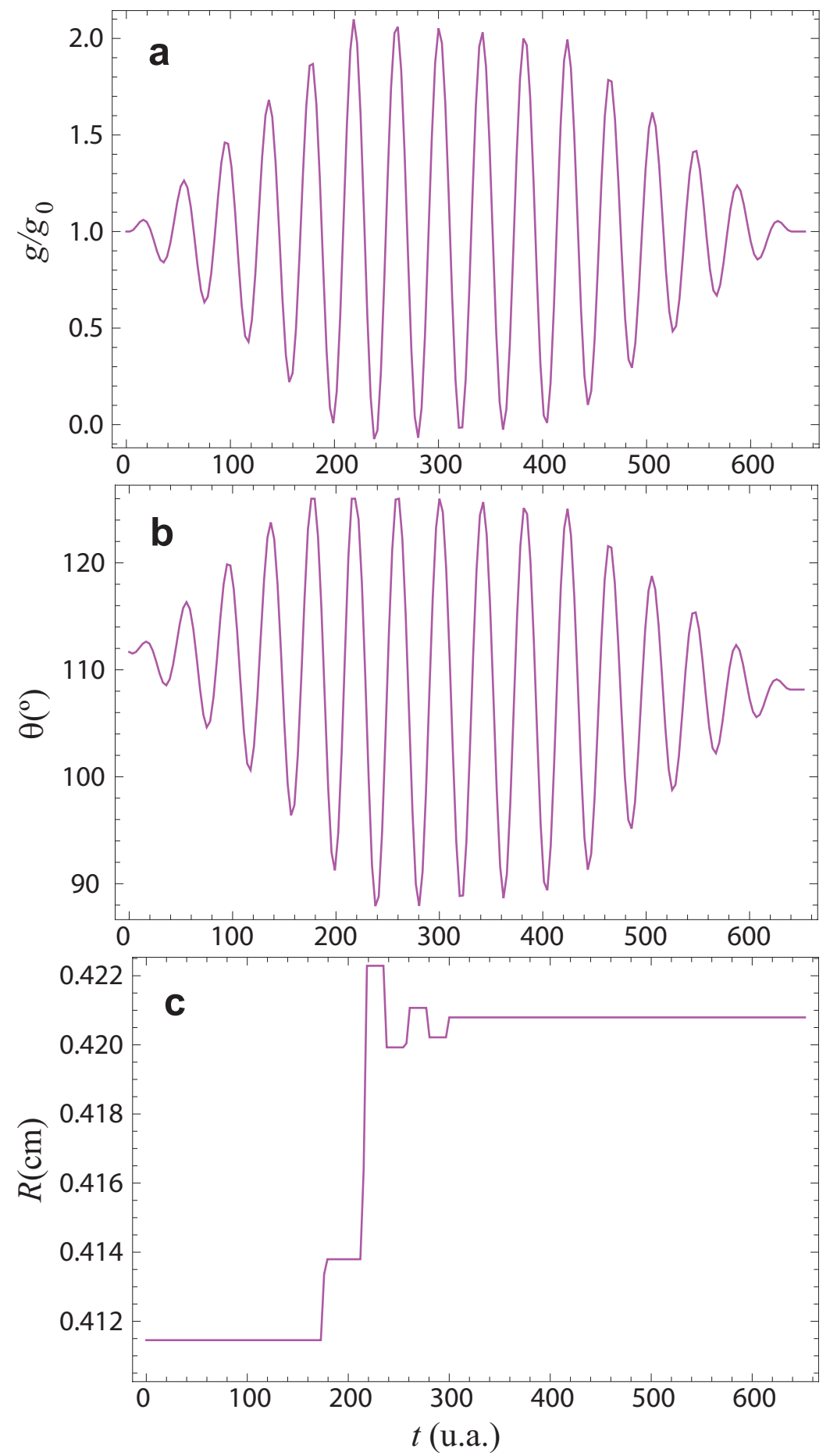

Figura 4.7: Resultados del método de simulación de vibraciones para una gota de agua de $V=150 \mu /$ sobre PTFEr. Ángulo de contacto inicial $\theta_{0}=111,71^{\circ}$. a) Función oscilante. b) Ángulos de contacto. c) Radio de la gota. 
4.7b para una gota de agua sobre PTFEr, y de este modo permita que el radio de la gota aumente y disminuya oscilando en torno a su valor final (figura 4.7c). Si se eligen amplitudes menores el sistema no alcanza el mismo estado final para todos los ángulos iniciales $\theta_{0}$. Desde un punto de vista práctico es importante que las oscilaciones del radio no tengan mucha amplitud pues esto disminuye el error en la estimación del valor final del radio. También es importante que la transición desde una amplitud de oscilación que permita alcanzar $\theta_{\text {re }}$ y $\theta_{\text {av }}$ y el movimiento de la línea triple hasta una amplitud menor en que la línea triple permanezca fija tenga lugar lo mas lentamente posible. La importancia de este hecho radica en que en el punto de transición se determina el radio final de la gota que nos permite obtener el ángulo de contacto final del proceso de vibración cuando $g=g_{0}$.

En el caso mostrado en la figura 4.7 se considera una gota de agua con un volumen $V=150 \mu \mathrm{l}$ sobre PTFEr con un ángulo de contacto inicial $\theta_{0}=111,71^{\circ}$. Para este sistema $\theta_{\mathrm{av}}=126^{\circ}$ y $\theta_{\mathrm{re}}=87,9^{\circ}$ [46]. La superficie de contacto inicial de la gota tiene forma circular y se mantiene circular durante el proceso de simulación. Tiendo en cuenta el método de simulación, las abscisas de la figura 4.7 representan una variable que indica cómo evoluciona el sistema. Aunque no se trata de la variable tiempo, por analogía se emplea la notación $t$, con unidades arbitrarias (u.a.). Como se puede observar en la figura $4.7 \mathrm{c}$, para $\theta_{0}=111,71^{\circ}$ con $g=g_{0}=9,8 \mathrm{~m} / \mathrm{s}^{2}$ la gota tiene un radio de contacto $R=0,4115 \mathrm{~cm}$. Este radio permanece constante hasta $t \simeq 180$ u.a. en que $\theta$ alcanza el valor máximo $\theta_{\mathrm{av}}$ (véase la figura $4.7 \mathrm{~b}$ ), en cuyo caso $R$ aumenta hasta un valor $R=0,414 \mathrm{~mm}$. El radio vuelve a aumentar cuando $\theta$ alcanza de nuevo el valor $\theta_{\mathrm{av}}$. En $t \simeq 240$ u.a. se alcanza por primera vez el valor $\theta_{\text {re }}$ (véase la figura $4.7 \mathrm{~b}$ ) y el radio disminuye. A continuación $R$ oscila hasta $t \simeq 300$ u.a. En ese momento, la amplitud de oscilación de $g$ (figura 4.7a) desciende por debajo del umbral que permite a $\theta$ alcanzar los valores de avance y retroceso y $R$ permanece constante. Al final del proceso ( $t \gtrsim 640$ u.a.), cuando $g=g_{0}$, el sistema alcanza el ángulo de contacto final, que en este caso es $\theta_{\text {vib }} \simeq 108^{\circ}$. El ángulo obtenido puede ser considerado como el más estable para esta situación pues es el que permite mayor amplitud de variación de $g$ (desde 0 a $2 g_{0}$, aproximadamente) sin que varíe el radio de contacto. 

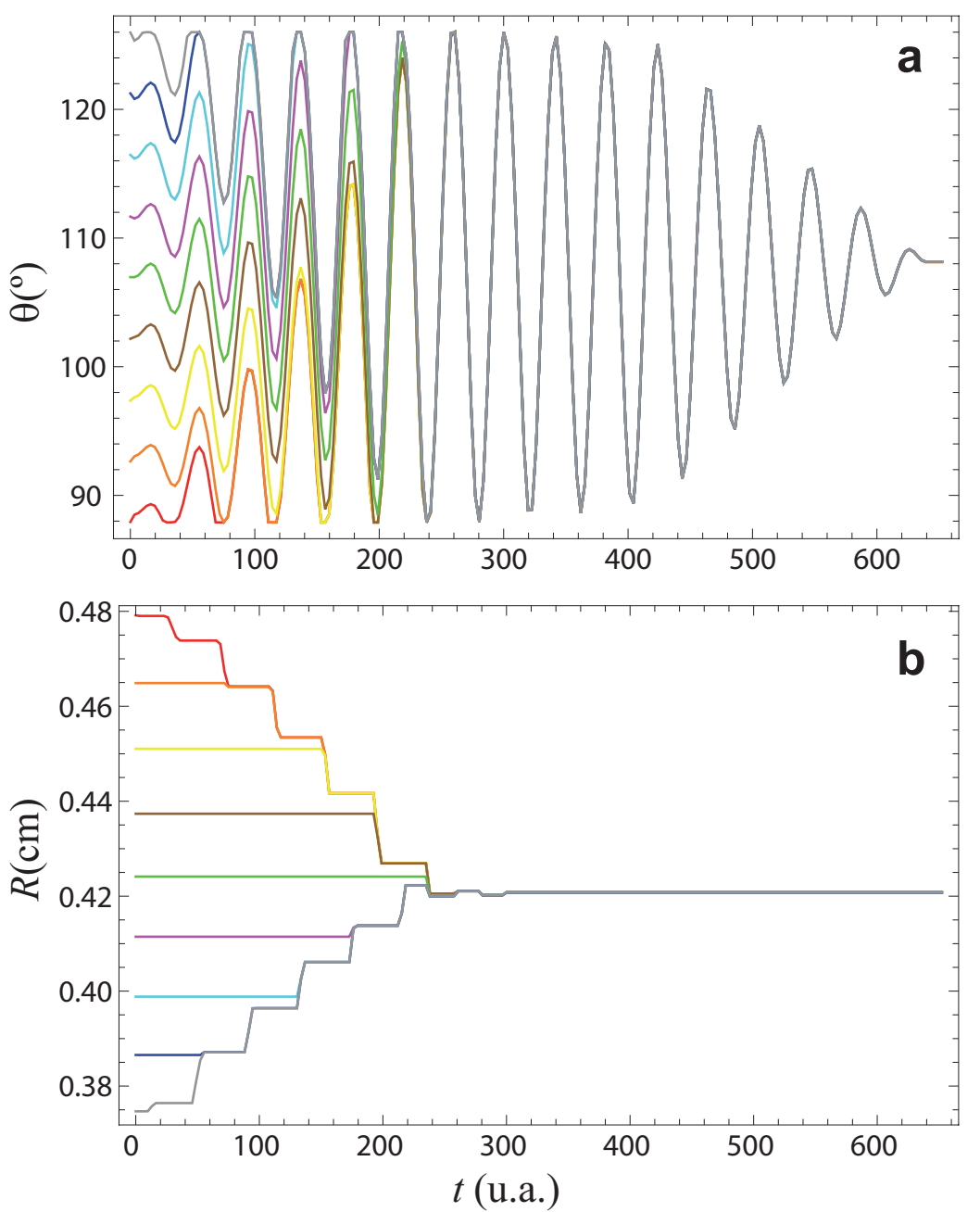

Figura 4.8: Resultados del método de simulación de vibraciones para una gota de agua de $V=150 \mu /$ sobre PTFEr. a) Ángulos de contacto y b) Radio de contacto. Ángulo inicial $\theta_{0}: 87,9^{\circ}$ rojo,

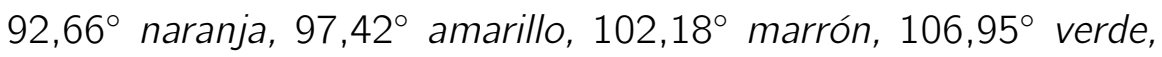
$111,71^{\circ}$ rosa, $116,47^{\circ}$ azul claro, $121,23^{\circ}$ azul oscuro y $126^{\circ}$ gris. 
La figura 4.8 considera el mismo sistema que la figura 4.7 pero con ángulos de contacto iniciales $\theta_{0}$ diferentes, como se puede ver en la figura 4.8a, donde también se observa que el ángulo de contacto final $\theta_{\text {vib }}$ varía muy poco. La figura $4.8 b$ muestra la evolución de los radios de contacto; como se puede observar, para cada valor de $\theta_{0}$ se tiene un valor inicial de $R$ diferente. Tras la oscilación todos los valores de $R$ son prácticamente iguales.

A la vista de los resultados presentados en la figura 4.8 es posible realizar la estimación del ángulo de contacto más estable por dos procedimientos ligeramente diferentes:

Método A: el ángulo de contacto más estable se determina realizando un promedio de los valores $\theta_{\text {vib }}$ obtenidos para los distintos ángulos de contacto iniciales considerados. Denotamos por $\theta_{\text {vib }}^{A}$ al resultado de este método.

Método B: Se representa el valor absoluto de la diferencia entre el valor inicial $\theta_{0}$ y el final $\theta_{\text {vib }}$ frente a $\theta_{0}$. Cuando ésta diferencia es mínima (idelamente sería cero), el valor de $\theta_{0}$ correspondiente coincide con el ángulo de contacto más estable pues es el que permanece inalterado tras la vibración. Este es el método experimental propuesto por Rodríguez-Valverde et al. [46]. Denotamos por $\theta_{\text {vib }}^{\mathrm{B}}$ al resultado de este método.

La figura 4.9 muestra un ejemplo de aplicación del método $\mathrm{B}$ a los resultados del sistema de la figura 4.8. Como se puede observar la intersección de las rectas de ajuste tiene lugar para un ángulo de contacto más estable $\theta_{\text {vib }}^{\mathrm{B}}=108,15^{\circ}$, con una diferencia $\left|\theta_{\text {vib }}-\theta_{0}\right|$ aproximadamente igual a $0^{\circ}$.

Desde un punto de vista práctico es de destacar que los datos de entrada para la simulación son los característicos del sistema líquido-superficie, es decir, ángulos de contacto de avance y retroceso, el volumen de la gota $V$, la tensión superficial $\sigma_{\mathrm{lv}}$ y la densidad del líquido $\rho$. Otros datos son el ángulo de contacto inicial $\theta_{0}$ y en este caso la frecuencia y amplitud de la función que genera la vibración. Para comprobar que ésta última es adecuada, se realiza una simulación previa y se verifica que la línea de contacto se mueve durante la vibración (o, de manera equivalente, que el radio de contacto cambia). Además, tras la simulación, se comprueba que el radio 


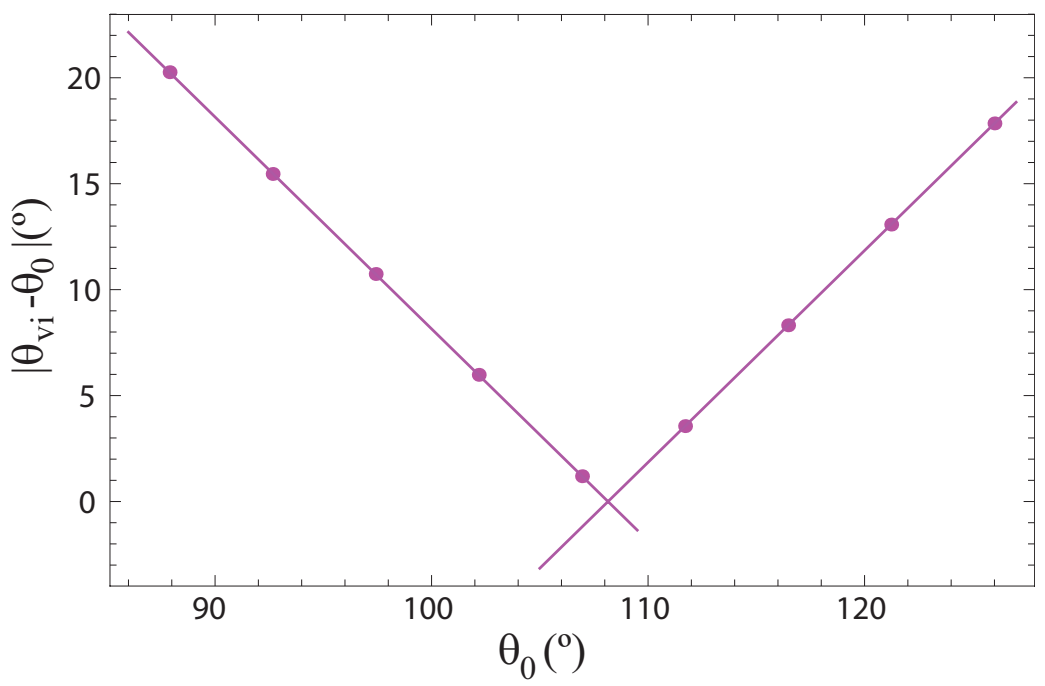

Figura 4.9: Valor absoluto de la diferencia entre los ángulos de contacto después de la vibración y los ángulos de contacto iniciales, en función del ángulo de contacto inicial. Simulación de vibración para una gota de agua de $V=150 \mu /$ sobre PTFEr. Las rectas son ajustes lineales de los datos. 
de contacto de la gota se estabiliza antes de que el proceso de vibración termine, evitando así que la gota quede "atrapada" en un estado metaestable y no se mida realmente el ángulo de contacto mas estable.

\subsubsection{Resultados}

En el cuadro 4.3 se presentan los resultados obtenidos por los dos métodos presentados previamente para el cálculo del ángulo de contacto más estable. Se han considerado gotas de agua de volumen $V=150 \mu \mathrm{l}$ sobre siete polímeros PP, PC, PTFE, PET, uPVC, PDMS, PDMS y PTFE, éste último con dos rugosidades diferentes. Se han escogido estos sistemas para poder comparar con los resultados obtenidos en el apartado 4.2 para el método de placa inclinable y con los resultados experimentales de Rodríguez-Valverde et al. $[45,46]$. Como se puede ver en el cuadro 4.3 los resultados de los métodos A y B son muy similares, como era de esperar. Por otro lado, se observa que en todos los casos los resultados de simulación son mayores que las estimaciones teóricas (4.1) y (4.2), aunque se mantienen bastante próximos salvo en el caso de PDMSs probablemente debido a la gran histéresis del sistema.

La figura 4.10 muestra el valor absoluto de la variación de los ángulos de contacto después de la vibración $\left|\theta_{\text {vib }}-\theta_{0}\right|$, frente al ángulo de contacto inicial $\theta_{0}$, para una gota de agua sobre uPVC y dos volúmenes diferentes, con el objetivo de estudiar la influencia del volumen en la estimación final del ángulo de contacto más estable tras la vibración. En la tabla 4.4 se recogen los resultados obtenidos. Se observa que prácticamente no existen diferencias entre los valores calculados.

\subsection{Comparación de resultados}

En la figura 4.11 se muestran de manera conjunta los resultados obtenidos mediante simulaciones de placa inclinable y vibraciones mecánicas de un substrato horizontal para un sistema agua sobre PTFEs. En la figura 4.11a) recogemos los datos procedentes de simulaciones de placa inclinable, representando la diferencia de los ángulos de inclinación críticos $\alpha_{2}-\alpha_{1}$ frente al ángulo de contacto inicial $\theta_{0}$, para 
Método de vibración: ángulo de contacto más estable

\begin{tabular}{ccccccc}
\hline Polímero & $\theta_{\mathrm{av}}\left(^{\circ}\right)$ & $\theta_{\mathrm{re}}\left(^{\circ}\right)$ & $\left\langle\theta>\left(^{\circ}\right)\right.$ & $\left\langle\theta_{\mathrm{cos}}>\left(^{\circ}\right)\right.$ & $\theta_{\mathrm{vib}}^{\mathrm{A}}\left({ }^{\circ}\right)$ & $\theta_{\mathrm{vib}}^{\mathrm{B}}\left({ }^{\circ}\right)$ \\
\hline PP & $100,7 \pm 1,6$ & $70 \pm 3$ & 85,35 & 85,51 & 86,60 & 86,61 \\
PC & $86,2 \pm 1,5$ & $48 \pm 2$ & 67,1 & 68,42 & 69,79 & 69,48 \\
PET & $80 \pm 2$ & $49,7 \pm 1,5$ & 64,85 & 65,78 & 66,78 & 66,79 \\
uPVC & $86 \pm 2$ & $50 \pm 2$ & 68 & 69,13 & 70,46 & 70,17 \\
PTFEs & $110 \pm 4$ & $95,6 \pm 1,0$ & 102,8 & 102,70 & 103,00 & 103,00 \\
PTFEr & $126 \pm 2$ & $87,9 \pm 1,3$ & 106,95 & 105,99 & 108,15 & 108,15 \\
PDMSs & $120,2 \pm 1,9$ & $65,2 \pm 1,9$ & 92,7 & 92,39 & 96,21 & 96,21 \\
\hline
\end{tabular}

Cuadro 4.3: Ángulos de contacto de avance y retroceso [46], ángulo de contacto más estable obtenido a partir de la media aritmética de estos valores (4.1) así como el valor obtenido de la media de los cosenos (4.2), y los resultados obtenidos mediante simulación para el método de vibración utilizando los procedimientos $A$ y $B$. Se considera una gota de agua de $150 \mu$ l sobre varios polímeros. 


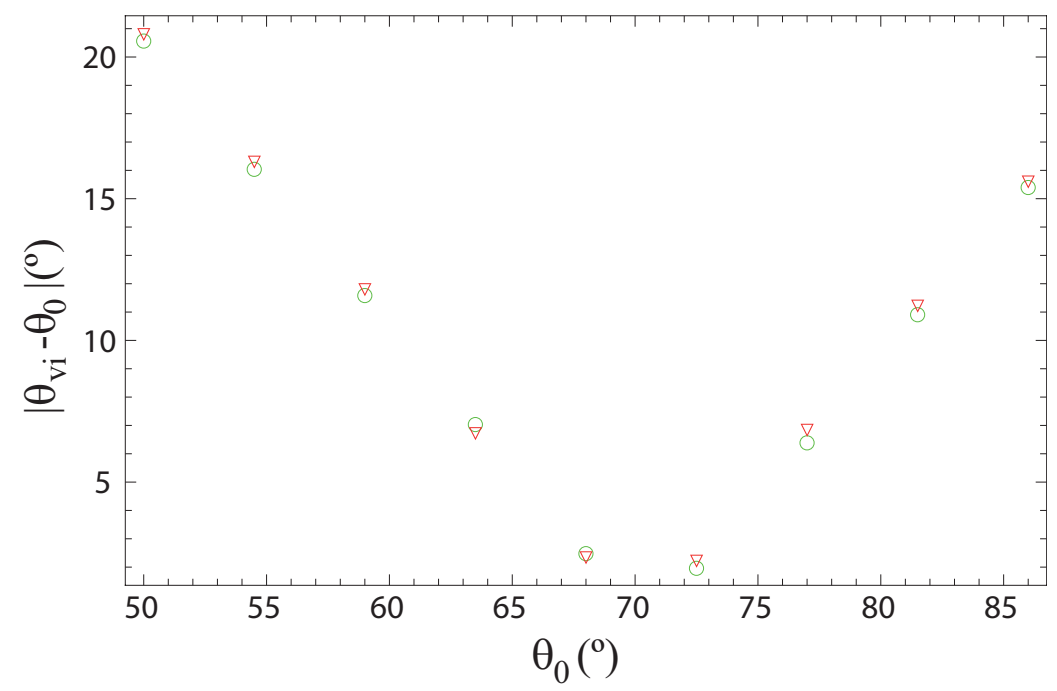

Figura 4.10: Valor absoluto de la variación de los ángulos de contacto después de la vibración en términos del ángulo de contacto inicial para una gota de agua sobre uPVC. Círculos verdes: $V=50 \mu l$, triángulos rojos: $V=150 \mu l$.

\begin{tabular}{ccc}
\hline Volumen $(\mu \mid)$ & $\theta_{\text {vib }}^{\mathrm{A}}\left(^{\circ}\right)$ & $\theta_{\text {vib }}^{\mathrm{B}}\left({ }^{\circ}\right)$ \\
\hline 50 & 70,56 & 70,52 \\
150 & 70,46 & 70,17 \\
\hline
\end{tabular}

Cuadro 4.4: Valor del ángulo de contacto más estable determinado mediante el método de vibración, para dos volúmenes diferentes de la gota de agua sobre uPVC; obtenidos por el método $A\left(\theta_{\mathrm{vib}}^{A}\right)$ y por el método $B\left(\theta_{\text {vib }}^{B}\right)$. 

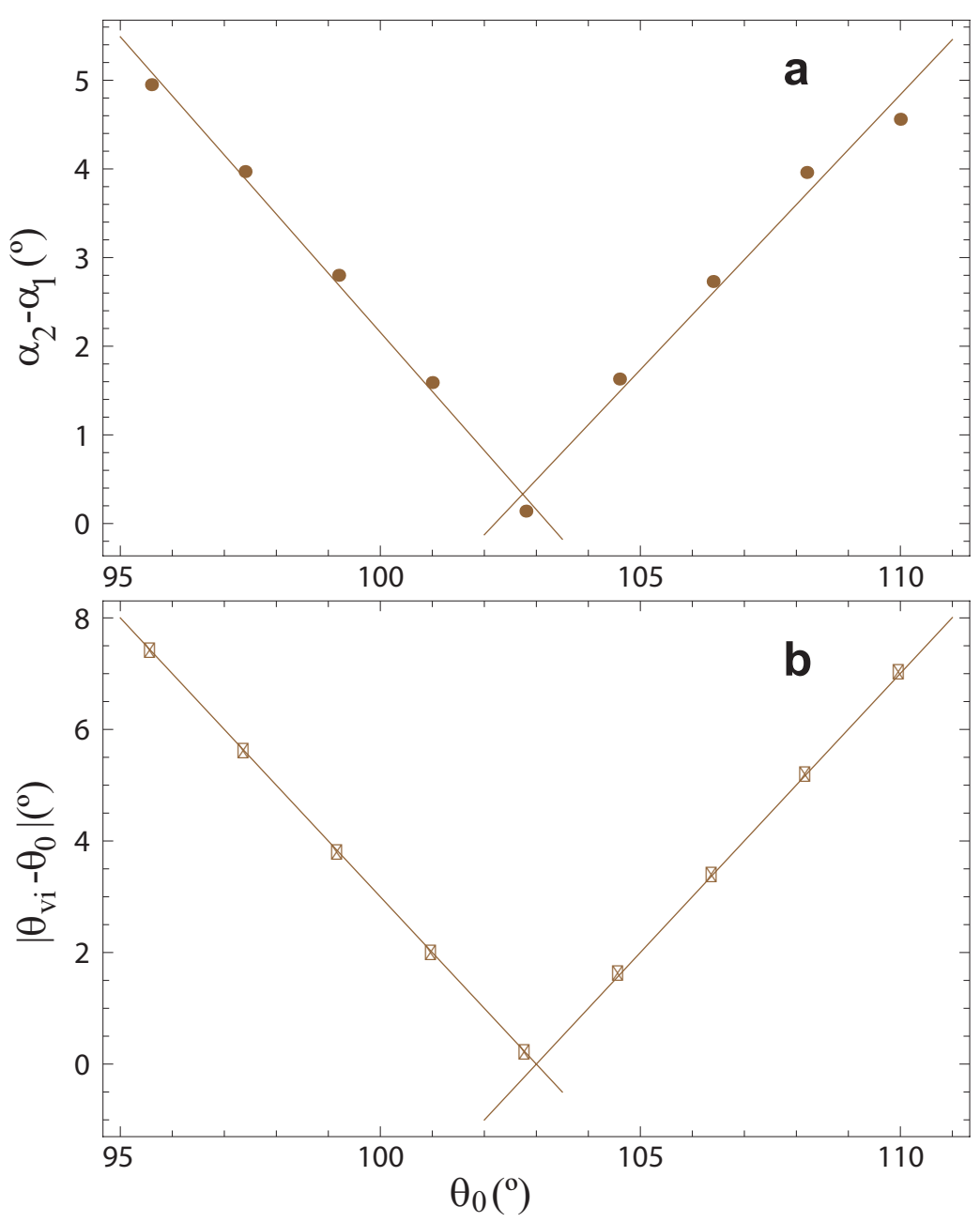

Figura 4.11: a) Diferencia de los ángulos de inclinación críticos frente al ángulo de contacto inicial, simulación de placa inclinable (círculos). b) Valor absoluto de la variación de los ángulos de contacto después de la vibración en términos del ángulo de contacto inicial, simulación de vibración (cuadrados). Ambas para una gota de agua de $V=150 \mu /$ sobre PTFEs. 
una gota de agua de $V=150 \mu$ l (círculos). Las rectas son ajustes lineales de los resultados.

En la figura 4.11b se representa el valor absoluto de la variación de los ángulos de contacto después de la vibración $\left|\theta_{\text {vib }}-\theta_{0}\right|$ en función del ángulo de contacto inicial $\theta_{0}$. En este caso el ángulo de contacto más estable $\theta_{\text {me }}$ se corresponde con aquel ángulo de contacto cuya configuración inicial da lugar a la mínima variación del ángulo de contacto tras la vibración $\left(\left|\theta_{\text {vib }}-\theta_{0}\right| \rightarrow 0\right)$. El ángulo de contacto más estable obtenido mediante el método de placa inclinable es $102,85^{\circ}$ y el valor obtenido con el método de vibraciones mecánicas es $103,00^{\circ}$. Como se puede comprobar los valores obtenidos por ambos métodos son muy próximos en este caso particular.

En el cuadro 4.5 se comparan los resultados de simulación de placa inclinable y de vibración mecánica, con los resultados experimentales de Montes et al. [46]. Se ha considerado en ambos casos el método B por ser el que utilizan Montes et al.. De este cuadro se pueden extraer las siguientes consideraciones:

- Las estimaciones por simulación para el ángulo de contacto más estable por medio del método de vibraciones son siempre ligeramente mayores que las del método de placa inclinable.

- En todos los casos existe buen acuerdo entre los resultados de simulación y los resultados obtenidos a partir de las expresiones $(4.1)(<\theta>)$ y (4.2) $\left(<\theta_{\cos }>\right)$. La mayor diferencia aparece para PDMSs en el resultado obtenido por la simulación del método de vibración. Como se comentó en el apartado 4.3.1 estas diferencias pueden ser debidas a la gran histéresis del sistema.

- La comparación entre los resultados experimentales y de simulación para el método de placa inclinada muestran un buen acuerdo en todos los casos excepto para uPVC. Para este sistema también existen grandes discrepancias entre los resultados experimentales y de simulación para el método de vibración. Estas diferencias indican la existencia de otros efectos en este sistema que no pueden ser explicados con el presente modelo de simulación.

- La comparación entre los resultados experimentales y de simulación para el método de vibración muestra, en general, mayores diferencias que el método 


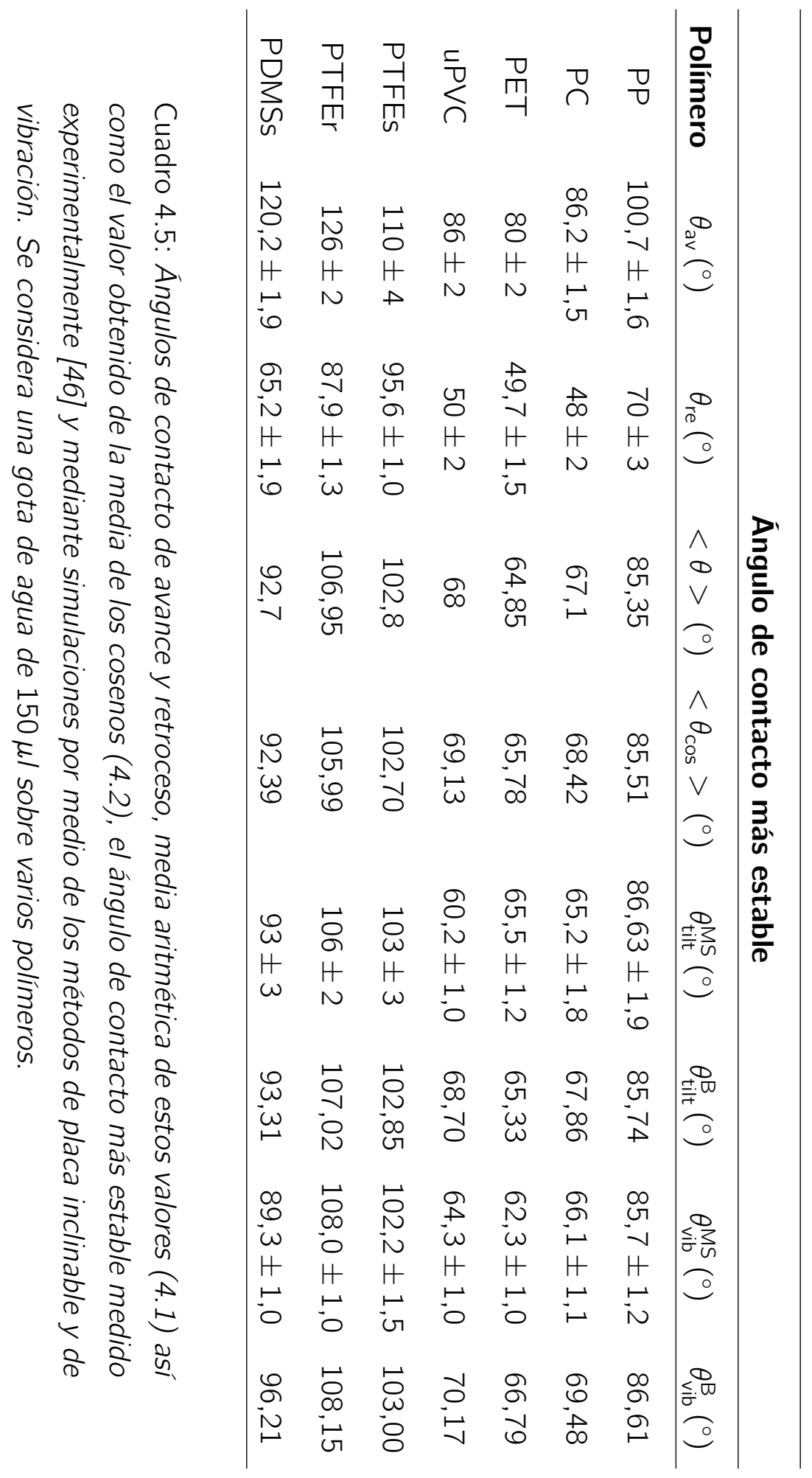


de placa inclinable. Este hecho parece indicar que el método de placa inclinable de Rodríguez-Valverde et al. [45] podría ser más adecuado que el método experimental de vibraciones mecánicas, puesto que el primer método da lugar a mayor acuerdo con simulación y con las estimaciones teóricas (4.1) y (4.2).

\subsection{Conclusiones}

En este capítulo se han expuesto dos caminos diferentes para medir el ángulo de contacto más estable de un sistema líquido-sólido, a partir de la gota mecánicamente más estable, con diferentes configuraciones de ángulos de contacto inicial. A partir de los trabajos de Rodríguez-Valverde et al. [45] y Montes et al. [46] se consideran dos métodos distintos de simulación: placa inclinable y vibración mecánica.

En el método de placa inclinable, se analiza el comportamiento al ser inclinado el sustrato de gotas sésiles con el mismo volumen pero con diferentes ángulos de contacto inicial. Utilizando el algoritmo desarrollado para Surface Evolver [65] se pueden medir los ángulos de inclinación críticos para cada gota. A partir de éstos, se identifica la gota más estable cuando el primer ángulo de inclinación crítica es máximo, y es entonces cuando ambos ángulos de inclinación crítica coinciden, es decir, el valor de $\theta_{0}$ para el cual se verifica $\alpha_{\mathrm{u}}-\alpha_{\mathrm{d}}=0$ nos permite obtener el ángulo de contacto más estable. Se comprueba, como ya había sido determinado experimentalmente [46], que para los sistemas estudiados no se observa una dependencia de los resultados de éste método con el volumen.

En el método de vibraciones mecánicas en gotas sésiles, también se consideran gotas de igual volumen pero con diferentes ángulos de contacto inicial. El ángulo de contacto más estable se identifica como aquel que no cambia después de la vibración, independientemente de la historia previa del sistema.

Finalmente se ha realizado una comparación de los resultados de simulación con datos experimentales [46] para el método de placa inclincable y para el método de vibración [45]. Se ha obtenido un buen acuerdo entre los resultados de simulación y las estimaciones teóricas dadas por las ecuaciones (4.1) y (4.2), así como los resultados experimentales para el método de placa inclinable. Se han encontrado 
mayores discrepancias con los resultados experimentales del método de vibraciones mecánicas. 


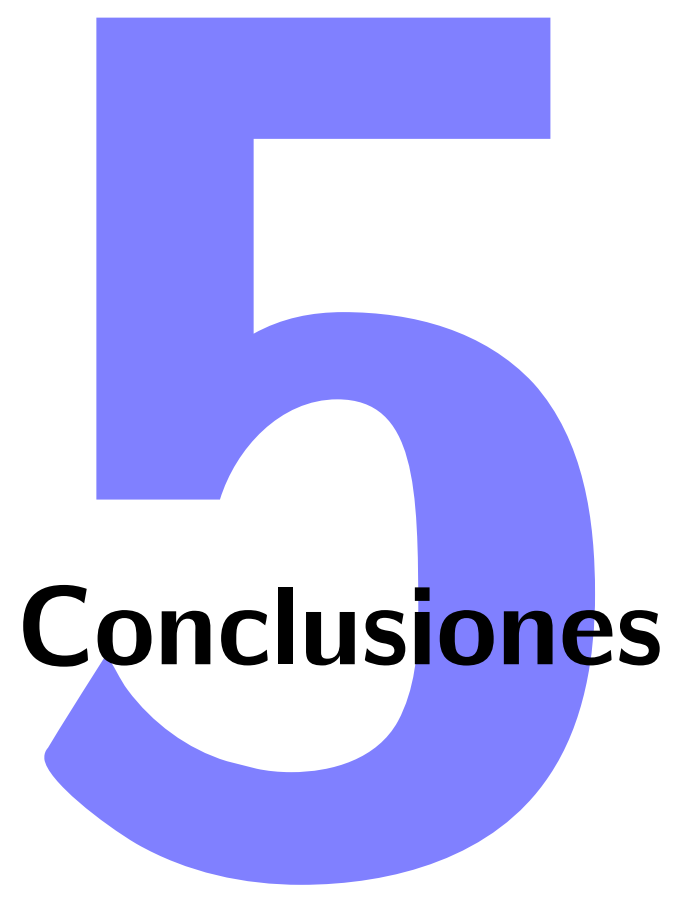

En este trabajo se han abordado distintos problemas relacionados con la histéresis de ángulos de contacto en gotas sésiles. El estudio se ha realizado tanto desde un punto de vista teórico como de simulación. La principales conclusiones de este trabajo son:

- Se ha propuesto un modelo sencillo para tratar la histéresis de ángulos de contacto en gotas sésiles axisimétricas. El modelo se basa en suponer que la línea triple permanece fija para ángulos de contacto comprendidos entre el de avance y el de retroceso, resolviendo la ecuación de Young-Laplace en coordenadas cilíndricas. Se ha obtenido un excelente acuerdo con resultados experimentales para ciclos de histéresis de ángulos de contacto.

- Se ha propuesto un algoritmo que permite simular histéresis de ángulos de contacto en Surface Evolver. El algoritmo está basado en la incorporación de ligaduras locales en el tratamiento variacional del problema. Las ligaduras locales tienen en cuenta la aparición de fuerzas de fricción.

- Sobre la base del algoritmo anterior, se ha propuesto un nuevo método para la medida de ángulos de contacto en Surface Evolver, basado en las fuerzas 
que actúan sobre la línea triple. El nuevo método es más preciso que la medida directa de las ángulos de contacto.

- La combinación del algoritmo de simulación con el nuevo método de medida ha permitido obtener resultados para gotas axisimétricas que muestra un excelente acuerdo tanto con resultados teóricos como experimentales para ciclos de histéresis de ángulos de contacto, incluso en situaciones con ángulos de retroceso dependientes del tiempo.

- A través del modelo implementado en Surface Evolver, se ha realizado un estudio de simulación del proceso en el cual una gota de líquido colocada inicialmente sobre una superficie horizontal se inclina gradualmente hasta que desliza por completo. Se ha encontrado que los resultados obtenidos mediante estas simulaciones en Surface Evolver presentan una gran dependencia con los ángulos de contacto iniciales de la gota. Así, la situación con el ángulo de contacto inicial igual a $\theta_{\mathrm{av}}$ es quizá la más próxima a los experimentos, mientras que un ángulo de contacto inicial igual a $\theta_{Y}=\left(\theta_{\mathrm{re}}+\theta_{\mathrm{av}}\right) / 2$ conduce a resultados previamente obtenidos por otros autores por métodos de elementos finitos.

- El análisis de los resultados para gotas sobre superficies gradualmente inclinadas ha permitido obtener una serie de relaciones entre fuerzas de retención, ángulo de inclinación y ángulos de contacto máximo y mínimo. Es de destacar la relación lineal existente entre el seno del ángulo de inclinación y los cosenos del ángulo de contacto máximo y mínimo. Se ha obtenido muy buen acuerdo al comparar con resultados experimentales.

- Un análisis de la deformación de la base de la gota en el caso $\theta_{\text {ini }}=\theta_{\text {av }}$ ha permitido establecer una serie de relaciones entre la relación de aspecto, el radio medio de la gota, su semiancho, el área de la base y la fuerza de retención. Estas relaciones pueden servir de guía para posteriores estudios experimentales.

- La simulaciones de gotas sobre superficies inclinadas han permitido identificar tres ángulos críticos en el problema: el ángulo de inclinación para el cual ocurre el movimiento incipiente de la parte posterior de la gota, $\alpha_{\mathrm{u}}$, el ángulo para 
el cual ocurre el movimiento incipiente de la parte frontal de la gota, $\alpha_{d}$, y el ángulo para el cual la gota comienza a deslizar, $\alpha_{\mathrm{c}}$.

- Se ha realizado un estudio de simulación del ángulo de contacto más estable a partir del método experimental de placa inclinable diseñado por RodríguezValverde et al. [45] que permite identificar esta ángulo a partir de los valores de $\alpha_{\mathrm{u}}$ y $\alpha_{\mathrm{d}}$ obtenidos para gotas con diferentes ángulos de contacto iniciales.

- Se ha desarrollado un nuevo método de simulación del ángulo de contacto más estable basado en el establecimiento de una pseudodinámica de vibración en una gota sésil. En este método, partiendo de diferentes condiciones iniciales, se alcanza el ángulo de contacto más estable tras someter a la gota a cambios armónicos en la aceleración debida a la gravedad.

- La comparación de resultados experimentales y de simulación para los dos métodos anteriores muestra en general un buen acuerdo entre los resultados de simulación y los datos experimentales correspondientes al método de placa inclinable, apareciendo mayores discrepancias con el método experimental de vibraciones mecánicas. 



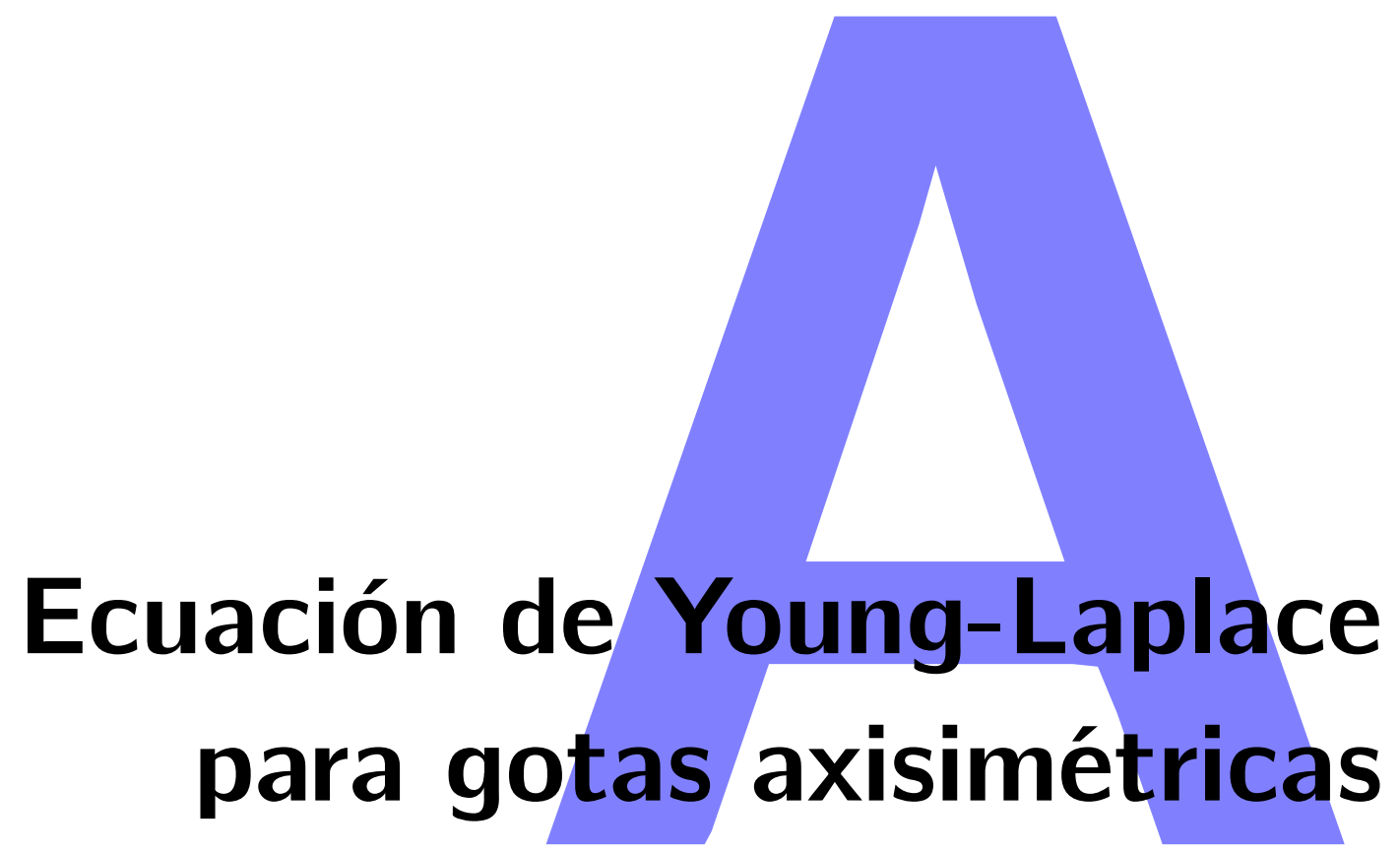

Pasamos a continuación a exponer la ecuación de Young-Laplace en coordenadas cilíndricas, apoyados en los trabajos de Del Rio el at. [9] y Gea-Jódar [8].

\section{A.1. Ecuación de Young-Laplace en coordenadas cilín- dricas}

Las gotas sésiles sobre una superficie plana, horizontal, lisa y homogénea de un sólido (una superficie ideal) presentan simetría axial, de manera que es posible describir la gota sésil como una interfaz de revolución $\Sigma$ en coordenadas cilíndricas. La parametrización de la superficie puede ser descrita por

$$
r_{\Sigma}(z, \varphi)=(R(z) \cos \varphi, R(z) \operatorname{sen} \varphi, z)
$$

donde $R, \varphi, z$ representan las coordenadas cilíndricas como se puede ver en la figura A.1: coordenada radial $R$, coordenada acimutal $\varphi$, coordenada axial $z$ y $\theta$ el ángulo tangencial del perfil de la gota. Es común tomar como origen de alturas el ápice de la gota, de manera que el radio medio de curvatura se convierte en el radio de curvatura (único dada la simetría de revolución de la gota) en el ápice. Situando el 


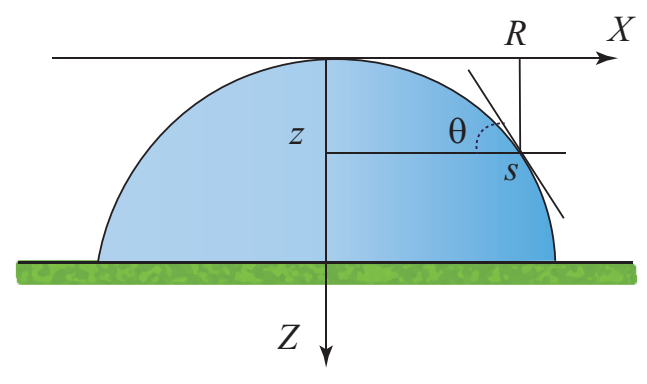

Figura A.1: Variables que se utilizan para expresar la ecuación de Young-Laplace en coordenadas cilíndricas.

eje de alturas (eje $z$ ) orientado hacia abajo, la ecuación de Young-Laplace adquiere la forma:

$$
\frac{1}{R(z) \sqrt{1+R^{\prime}(z)^{2}}}-\frac{R^{\prime \prime}(z)}{\left(1+R^{\prime}(z)^{2}\right)^{3 / 2}}=\frac{2}{r_{0}}+\frac{z}{l_{0}^{2}},
$$

donde $r_{0}$ es el radio de curvatura en el ápice (1.13) y $l_{0}$ es la longitud de capilaridad del líquido (1.14). La consecuencia de esta orientación del eje $z$ se refleja en el signo de la dependencia de la diferencia de presión con la altura.

A diferencia de la ecuación de Young-Laplace en su forma general, (A.2) es una ecuación diferencial de segundo orden ordinaria que se puede simplificar y convertir en un sistema de ecuaciones diferenciales de primer orden. Para ello se introducen dos nuevas variables $\theta$ y $s$ de acuerdo a las definiciones siguientes:

1. La variable $\theta$ es el ángulo que forman la normal a la superficie y la vertical, el cual, para una gota sésil, se convierte en el ángulo de contacto. Queda definida por la relación:

$$
\tan \theta(z)=\frac{1}{R^{\prime}(z)},
$$

que puede interpretarse como la pendiente que tiene un punto de la interfaz respecto a la horizontal. Por lo tanto:

$$
1+R^{\prime}(z)^{2}=\frac{1}{\operatorname{sen}^{2} \theta} ;
$$


obteniendo

$$
R^{\prime \prime}(z)=-\frac{1}{\operatorname{sen}^{2} \theta} \theta^{\prime}(z)
$$

Utilizando la expresión (A.4), el primer sumando de la ecuación de YoungLaplace (A.2) queda

$$
\frac{1}{R(z) \sqrt{1+R^{\prime}(z)^{2}}}=\frac{\operatorname{sen} \theta}{R(z)} .
$$

Y partiendo de la expresiones (A.4) y (A.5) el segundo sumando de la ecuación de Young-Laplace (A.2) resulta

$$
\frac{R^{\prime \prime}(z)}{\left(1+R^{\prime}(z)\right)^{3 / 2}}=-(\operatorname{sen} \theta) \theta^{\prime}(z) .
$$

Finalmente, introduciendo las expresiones (A.6) y (A.7) en la ecuación de Young-Laplace (A.2), se obtiene:

$$
\frac{\operatorname{sen} \theta}{R(z)}+(\operatorname{sen} \theta) \theta^{\prime}(z)=\frac{2}{r_{0}}+\frac{z}{l_{0}^{2}}
$$

2. Interpretando el parámetro s como la longitud del arco, se define:

$$
s(z)=\int_{0}^{z} \sqrt{1+R^{\prime}(z)^{2}} d z .
$$

De acuerdo a esta definición y a la de $\theta(A .4)$ se puede escribir

$$
\frac{d s}{d z}=\sqrt{1+R^{\prime}(z)^{2}}=\frac{1}{\operatorname{sen} \theta}
$$

y por tanto

$$
z^{\prime}(s)=\operatorname{sen} \theta(s)
$$

Utilizando las ecuaciones (A.8), (A.3) y (A.11) se llega a

$$
\left\{\begin{array}{l}
\theta^{\prime}(s)=\frac{2}{r_{0}}+\frac{z(s)}{l_{0}^{2}}-\frac{\operatorname{sen} \theta(s)}{R(s)} \\
z^{\prime}(s)=\operatorname{sen} \theta(s) \\
R^{\prime}(s)=\cos \theta(s) .
\end{array}\right.
$$


Este sistema se puede integrar con las condiciones iniciales, válidas para el ápice de la gota:

$$
\left\{\begin{array}{l}
\theta(0)=0 \\
z(0)=0 \\
R(0)=0 .
\end{array}\right.
$$

El radio de curvatura del ápice depende del volumen de la gota y de su extensión sobre la superficie.

En la región de contacto, las variables $\theta, z, R$ adoptan valores característicos que coinciden respectivamente con el ángulo de contacto $\theta_{c}$, la altura $z_{\mathrm{c}}$ y el radio de contacto de la gota $R_{\mathrm{c}}$.

Aunque el área y el volumen no son necesarios en principio, se han incluido aquí debido a su importancia en el caso particular de este estudio. $Y$ así, se añaden al sistema anterior (A.12) para obtener, tras la integración, el área de la interfaz líquidovapor y el volumen de la gota.

3. Área del interfaz líquido-vapor $A$ :

$$
A(z)=\int_{0}^{z} \int_{0}^{2 \pi} \sqrt{1+R^{\prime}(z)^{2}} R(z) d \varphi d z=\int_{0}^{z} 2 \pi R(z) \sqrt{1+R^{\prime}(z)^{2}} d z,
$$

por lo tanto, teniendo en cuenta la expresión (A.4)

$$
\frac{d A(z)}{d z}=2 \pi R(z) \sqrt{1+R^{\prime}(z)^{2}}=2 \pi R(z) \frac{1}{\operatorname{sen} \theta},
$$

que unido a la ecuación (A.11) permite expresar: $A^{\prime}(s)=A^{\prime}(z) z^{\prime}(s)=2 \pi R(s)$.

4. Volumen de la gota $V$ :

$$
V(z)=\int_{0}^{z} \int_{0}^{R(z)} \int_{0}^{2 \pi} R d \varphi d \rho d z=\int_{0}^{z} \pi R(z)^{2} d z
$$

por tanto

$$
\frac{d V(z)}{d z}=\pi R^{2}(z)
$$

o bien

$$
V^{\prime}(s)=\frac{d V(z)}{d z} \frac{d z}{d s}=\pi R(s)^{2} z^{\prime}(s)
$$


que, de nuevo, unido a la ecuación (A.11) permite deducir

$$
V^{\prime}(s)=V^{\prime}(z) z^{\prime}(s)=\pi R(s)^{2} \operatorname{sen} \theta(s)
$$

De este modo la ecuación de Young-Laplace en coordenadas cilíndricas resulta ser un sistema de ecuaciones, que se puede escribir, junto con las condiciones iniciales [9]

$$
\begin{cases}\theta^{\prime}(s)=\frac{2}{r_{0}}+\frac{z(s)}{I_{0}^{2}}-\frac{\operatorname{sen} \theta(s)}{R(s)} ; & \theta(0)=0 \\ z^{\prime}(s)=\operatorname{sen} \theta(s) ; & z(0)=0 \\ R^{\prime}(s)=\cos \theta(s) ; & R(0)=0 \\ A^{\prime}(s)=2 \pi R(s) ; & A(0)=0 \\ V^{\prime}(s)=\pi R(s)^{2} \operatorname{sen} \theta(s) ; & V(0)=0\end{cases}
$$

y la condición de contorno en el contacto

$$
\left\{\begin{array}{l}
V_{C}=V\left(s_{C}\right)=V \\
\theta_{C}=\theta\left(s_{C}\right)=\arccos \left(\frac{\sigma_{\mathrm{sv}}-\sigma_{\mathrm{sl}}}{\sigma_{\mathrm{lv}}}\right)
\end{array}\right.
$$





\section{Bibliografía}

[1] T. Young. An essay on the cohesion of fluids. Philosophical Transactions of the Royal Society of London 95, 65-87 (1805).

[2] P. S. Laplace. "Traité de mécanique céleste", tomo 4, Supplement to Book 10. Gauthier-Villars, Paris (1806).

[3] J. Berthier. "Microdrops and Digital Microfluidics: Processing, Development, and Applications". William Andrew Publishing, Norwich, New York (2007).

[4] D. Quere. Non-sticking drops. Reports on Progress in Physics 68, 2495-2532 (2005).

[5] B. Krasovitski y A. Marmur. Drops down the hill: Theoretical study of limiting contact angles and the hysteresis range on a tilted plate. Langmuir 21, 38813885 (2005).

[6] K. A. Brakke. "Surface evolver manual" (2008).

[7] J. N. Israelachvili. "Intermolecular and Surface Forces". Academic Press, Boston (1992).

[8] P. M. Gea-Jódar. "Mojado en condiciones de no-equilibrio sobre superficies reales". Tesis Doctoral, Universidad de Granada (2006).

[9] O. I. del Río y A. W. Neumann. Axisymmetric drop shape analysis: Computational methods for the measurement of interfacial properties from the shape and dimensions of pendant and sessile drops. Journal of Colloid and Interface Science 196, 136 - 147 (1997).

[10] S. S. Susnar, P. Chen, O. del Rio y A. W. Neumann. Surface tension response to area changes using axisymmetricdrop shape analysis. Colloids and Surfaces A: Physicochemical and Engineering Aspects 116, 181-194 (1996). 
[11] Y. Rotenberg, L. Boruvka y A. W. Neumann. Determination of surface tension and contact angle from the shapes of axisymmetric fluid interfaces. Journal of Colloid and Interface Science 93, 169 - 183 (1983).

[12] F. K. Skinner, Y. Rotenberg y A. W. Neumann. Contact angle measurements from the contact diameter of sessile drops by means of a modified axisymmetric drop shape analysis. Journal of Colloid and Interface Science 130, 25-34 (1989).

[13] E. Moy, P. Cheng, Z. Policova, S. Treppo, D. Y. Kwok, D. R. Mack, P. M. Sherman y A. W. Neumann. Measurement of contact angles from the maximum diameter of non-wetting drops by means of a modified axisymmetric drop shape analysis. Colloids and Surfaces 58, 215-227 (1991).

[14] R. N. Wenzel. Resistance of solid surfaces to wetting by water. Industrial \& Engineering Chemistry 28, 988-994 (1936).

[15] J. Berthier y P. Silberzan. "Microfluidics for Biotechnology". Artech House, Norwood, Massachusetts (2005).

[16] A. B. D. Cassie. Contact angles. Discussions of the Faraday Society 3, 11-16 (1948).

[17] J. Berthier, P. Dubois, P. Clementz, P. Claustre, C. Peponnet y Y. Fouillet. Actuation potentials and capillary forces in electrowetting based microsystems. Sensors and Actuators A: Physical 134, 471 - 479 (2007).

[18] J. Berthier, P. Clementz, O. Raccurt, D. Jary, P. Claustre, C. Peponnet y Y. Fouillet. Computer aided design of an ewod microdevice. Sensors and Actuators A: Physical 127, $283-294$ (2006).

[19] C. Monnereau, N. Pittet y D. Weaire. An analysis of surface curvature and growth laws for foam cells using the surface evolver. Europhysics Letters $\mathbf{5 2}$, 361-367 (2000). 
[20] N. A. Patankar y Y. Chen. Numerical simulation of droplet shapes on rough surfaces. En "Proc. Fifth Int. Conference on Modeling and Simulations of Microsystems (MSM 2002)", páginas 116-119 (2002).

[21] M. Rayner, G. Trägårdh, C. Trägårdh y P. Dejmek. Using the surface evolver to model droplet formation processes in membrane emulsification. Journal of Colloid and Interface Science 279, 175 - 185 (2004).

[22] S. J. Cox. A viscous froth model for dry foams in the surface evolver. Colloids and Surfaces A: Physicochemical and Engineering Aspects 263, 81 - 89 (2005).

[23] S. Brandon, A. Wachs y A. Marmur. Simulated contact angle hysteresis of a three-dimensional drop on a chemically heterogeneous surface: A numerical example. Journal of Colloid and Interface Science 191, 110 - 116 (1997).

[24] J. Lienemann, A. Greiner y J. G. Korvink. Modeling, simulation, and optimization of electrowetting. Computer-Aided Design of Integrated Circuits and Systems, IEEE Transactions on 25, 234-247 (2006).

[25] C. Dorrer y J. Rühe. Contact line shape on ultrahydrophobic post surfaces. Langmuir 23, 3179-3183 (2007).

[26] C. Dorrer y J. Rühe. Condensation and wetting transitions on microstructured ultrahydrophobic surfaces. Langmuir 23, 3820-3824 (2007).

[27] C. Dorrer y J. Rühe. Drops on microstructured surfaces coated with hydrophilic polymers: Wenzel's model and beyond. Langmuir 24, 1959-1964 (2008).

[28] P. S. H. Forsberg, C. Priest, M. Brinkmann, R. Sedev y J. Ralston. Contact line pinning on microstructured surfaces for liquids in the wenzel state. Langmuir 26, 860-865 (2010).

[29] T.-H. Chou, S.-J. Hong, Y.-J. Sheng y H.-K. Tsao. Drops sitting on a tilted plate: Receding and advancing pinning. Langmuir 28, 5158-5166 (2012).

[30] A. Marmur. Soft contact: measurement and interpretation of contact angles. Soft Matter 2, 12-17 (2006). 
[31] N. K. Adam y G. Jessop. Angles of contact and polarity of solid surfaces. Journal of the Chemical Society, Transactions 127, 1863 (1925).

[32] E. Wolfram y R. Faust. "Wetting, Spreading and Adhesion", capítulo Liquid drops on a tilted plate, contact angle hysteresis and the young contact angle, páginas 213-222. Academic Press, London (1978).

[33] R. A. Brown, F. M. Orr y L. E. Scriven. Static drop on an inclined plate: Analysis by the finite element method. Journal of Colloid and Interface Science 73, $76-87$ (1980).

[34] F. Milinazzo y M. Shinbrot. A numerical study of a drop on a vertical wall. Journal of Colloid and Interface Science 121, 254 - 264 (1988).

[35] E. B. Dussan V. y R. T. P. Chow. On the ability of drops or bubbles to stick to non-horizontal surfaces of solids. Journal of Fluid Mechanics 137, 1-29 (1983).

[36] E. B. Dussan V. On the ability of drops or bubbles to stick to non-horizontal surfaces of solids. pt. 2: small drops or bubbles having contact angles of arbitrary size. Journal of Fluid Mechanics 151, 1-20 (1985).

[37] S. D. Iliev. Iterative method for the shape of static drops. Computer Methods in Applied Mechanics and Engineering 126, 251 - 265 (1995).

[38] S. D. Iliev. Static drops on an inclined plane: Equilibrium modeling and numerical analysis. Journal of Colloid and Interface Science 194, 287 - 300 (1997).

[39] P. Dimitrakopoulos y J. J. L. Higdon. On the gravitational displacement of three-dimensional fluid droplets from inclined solid surfaces. Journal of Fluid Mechanics 395, 181-209 (1999).

[40] J. F. Joanny y P. G. de Gennes. A model for contact angle hysteresis. Journal of Chemical Physics 81, 552-562 (1984).

[41] P. G. de Gennes. Wetting: statics and dynamics. Reviews of Modern Physics 57, 827-863 (1985). 
[42] K. A. Brakke. The surface evolver. Experimental Mathematics 1, 141-165 (1992).

[43] C. N. C. Lam, R. Wu, D. Li, M. L. Hair y A. W. Neumann. Study of the advancing and receding contact angles: liquid sorption as a cause of contact angle hysteresis. Advances in Colloid and Interface Science 96, 169-191 (2002).

[44] E. I. Vargha-Butler, E. Kiss, C. N. C. Lam, Z. Keresztes, E. Kálmán, L. Zhang y A. W. Neumann. Wettability of biodegradable surfaces. Colloid and Polymer Science 279, 1160-1168 (2001).

[45] M. A. Rodriguez-Valverde, F. J. Montes Ruiz-Cabello y M. A. Cabrerizo-Vilchez. A new method for evaluating the most-stable contact angle using mechanical vibration. Soft Matter 7, 53-56 (2011).

[46] F. J. Montes Ruiz-Cabello, M. A. Rodriguez-Valverde y M. Cabrerizo-Vilchez. A new method for evaluating the most stable contact angle using tilting plate experiments. Soft Matter 7, 10457-10461 (2011).

[47] R. Tadmor. Line energy and the relation between advancing, receding, and young contact angles. Langmuir 20, 7659-7664 (2004).

[48] G. Macdougall y C. Ockrent. Surface energy relations in liquid/solid systems. i. the adhesion of liquids to solids and a new method of determining the surface tension of liquids. Proceedings of the Royal Society of London. Series A. Mathematical and Physical Sciences 180, 151-173 (1942).

[49] Y. I. Frenkel. On the behavior of liquid drops on a solid surface. 1. the sliding of drops on an inclined surface. Journal of Experimental and Theoretical Physics 18, 659 (1948).

[50] J. J. Bikerman. Sliding of drops from surfaces of different roughnesses. Journal of Colloid Science 5, 349 - 359 (1950). 
[51] C. G. L. Furmidge. Studies at phase interfaces. i. the sliding of liquid drops on solid surfaces and a theory for spray retention. Journal of Colloid Science 17, $309-324$ (1962).

[52] B. K. Larkin. Numerical solution of the equation of capillarity. Journal of Colloid and Interface Science 23, 305 - 312 (1967).

[53] C. W. Extrand y A. N. Gent. Retention of liquid drops by solid surfaces. Journal of Colloid and Interface Science 138, 431 - 442 (1990).

[54] C. W. Extrand y Y. Kumagai. Liquid drops on an inclined plane: The relation between contact angles, drop shape, and retentive force. Journal of Colloid and Interface Science 170, 515 - 521 (1995).

[55] A. I. ElSherbini y A. M. Jacobi. Retention forces and contact angles for critical liquid drops on non-horizontal surfaces. Journal of Colloid and Interface Science 299, $841-849$ (2006).

[56] M. Bouteau, S. Cantin, F. Benhabib y F. Perrot. Sliding behavior of liquid droplets on tilted langmuir-blodgett surfaces. Journal of Colloid and Interface Science 317, 247 - 254 (2008).

[57] D. Quere, M. Azzopardi y L. Delattre. Drops at rest on a tilted plane. Langmuir 14, 2213-2216 (1998).

[58] A. I. ElSherbini y A. M. Jacobi. Liquid drops on vertical and inclined surfaces: li. a method for approximating drop shapes. Journal of Colloid and Interface Science 273, 566 - 575 (2004).

[59] A. I. ElSherbini y A. M. Jacobi. Liquid drops on vertical and inclined surfaces: I. an experimental study of drop geometry. Journal of Colloid and Interface Science 273, 556 - 565 (2004).

[60] E. Pierce, F. J. Carmona y A. Amirfazli. Understanding of sliding and contact angle results in tilted plate experiments. Colloids and Surfaces A: Physicochemical and Engineering Aspects 323, 73 - 82 (2008). 
[61] P. S. Yadav, P. Bahadur, R. Tadmor, K. Chaurasia y A. Leh. Drop retention force as a function of drop size. Langmuir 24, 3181-3184 (2008).

[62] R. Tadmor y P. S. Yadav. As-placed contact angles for sessile drops. Journal of Colloid And Interface Science 317, 241-246 (2008).

[63] K. Kawasaki. Study of wettability of polymers by sliding of water drop. Journal of Colloid Science 15, $402-407$ (1960).

[64] M. J. Santos y J. A. White. Theory and simulation of angular hysteresis on planar surfaces. Langmuir 27, 14868-14875 (2011).

[65] M. J. Santos, S. Velasco y J. A. White. Simulation analysis of contact angles and retention forces of liquid drops on inclined surfaces. Langmuir 28, 1181911826 (2012).

[66] C. L. Moraila-Martínez, F. J. M. Ruiz-Cabello, M. A. Cabrerizo-Vílchez y M. A. Rodríguez-Valverde. The effect of contact line dynamics and drop formation on measured values of receding contact angle at very low capillary numbers. Colloids and Surfaces A: Physicochemical and Engineering Aspects 404, 63 69 (2012).

[67] H. Xu, Z. Yuan, J. Lee, H. Matsuura y F. Tsukihashi. Contour evolution and sliding behavior of molten sn-ag-cu on tilting cu and al2o3 substrates. Colloids and Surfaces A: Physicochemical and Engineering Aspects 359, 1 - 5 (2010).

[68] Z. Moser, W. Gasior, J. Pstruś y S. Ksiezarek. Surface-tension measurements of the eutectic alloy (ag-sn 96.2 at. \%) with cu additions. Journal of electronic materials 31, 1225-1229 (2002).

[69] V. Berejnov y R. E. Thorne. Effect of transient pinning on stability of drops sitting on an inclined plane. Physical Review E 75, 066308 (2007).

[70] T. S. Meiron, A. Marmur y I. S. Saguy. Contact angle measurement on rough surfaces. Journal of Colloid and Interface Science 274, 637 - 644 (2004). 
[71] C. Andrieu, C. Sykes y F. Brochard. Average spreading parameter on heterogeneous surfaces. Langmuir 10, 2077-2080 (1994).

[72] E. L. Decker, B. Frank, Y. Suo y S. Garoff. Physics of contact angle measurement. Colloids and Surfaces A: Physicochemical and Engineering Aspects 156, 177 - 189 (1999).

[73] S. Mettu y M. K. Chaudhury. Stochastic relaxation of the contact line of a water drop on a solid substrate subjected to white noise vibration: Roles of hysteresis. Langmuir 26(11), 8131-8140 (2010).

[74] F. J. Montes Ruiz-Cabello, M. A. Rodríguez-Valverde y M. A. Cabrerizo-Vílchez. Comparison of the relaxation of sessile drops driven by harmonic and stochastic mechanical excitations. Langmuir 27, 8748-8752 (2011).

[75] G. Wolansky y A. Marmur. Apparent contact angles on rough surfaces: the wenzel equation revisited. Colloids and Surfaces A: Physicochemical and Engineering Aspects 156, 381 - 388 (1999). 


\section{Îndice de figuras}

1.1. Radio de curvatura y círculo osculante. Imagen tomada de [3] . . 25

1.2. Esquema de una gota de líquido inmersa en un fluido; inicialmente, el radio de la gota es $R$ y su área superficial es $A_{0}$. Un aumento de su radio en una cantidad $d R$ se corresponde con un nuevo área superficial $A_{1}$ y una presión $p_{1} \ldots \ldots \ldots \ldots \ldots$

1.3. Cuando el líquido se extiende como una película sobre la superficie sólida se habla de mojado total.

1.4. Esquema de la región de contacto de una gota depositada sobre la superficie de un sólido. Las tensiones interfaciales son representadas vectorialmente tangentes a su interfaz. A partir de la componente horizontal del equilibrio de fuerzas se deduce la ecuación de Young.

1.5. a) Superficie hidrófila con $\theta_{Y}<90^{\circ}$ y b) superficie hidrófoba con $\theta_{Y}>90^{\circ}$.

1.6. Imagen de un momento de la evolución de una gota durante la simulación en Surface Evolver.

1.7. Ejemplo sencillo de un programa en Surface Evolver

2.1. Diagrama de flujo del algoritmo utilizado para obtener un ciclo de histéresis de una gota de líquido, resolviendo la ecuación de YoungLaplace en coordenadas cilíndricas.

2.2. Ciclo de histéresis de una gota de agua sobre una superficie de silicio recubierta de ácido poliláctico, obtenido resolviendo la ecuación de Young-Laplace (2.3) en coordenadas cilíndricas, entre $V_{\text {ini }}=0,03 \mathrm{~cm}^{3}$ y $V_{\text {fin }}=0,118 \mathrm{~cm}^{3}$. a) Evolución temporal del volumen. b) Evolución temporal del radio de contacto. c) Evolución temporal del ángulo de contacto. 
2.3. Ciclo de histéresis para una gota de agua sobre una superficie de silicio recubierta de ácido poliláctico. a) Ángulo de contacto $\theta$ frente al volumen $V$. b) Radio de la línea de contacto $R$ frente al volumen $V$. c) Fuerza de fricción adimensional $f_{\theta} / \sigma_{\mathrm{lv}}$ frente al volumen $V$. .

2.4. Diagrama de flujo del algoritmo utilizado en Surface Evolver. . . . .

2.5. Valor medio del ángulo de contacto para una gota de agua sobre una superficie de silicio recubierta de ácido poliláctico frente al volumen en una situación de avance. Líneas continuas: resultado del nuevo método basado en la medida de las fuerzas. Otras líneas: resultado de la medida directa de los ángulos de contacto $N=24$ (línea de puntos), $N=48$ (línea discontinua), $N=96$ (línea punto-raya), y $N=192$ (línea punto-punto-raya) . . . . . . . . . . . . .

2.6. Valor medio del ángulo de contacto para una gota de agua sobre una superficie de silicio recubierta de ácido poliláctico frente al valor de la longitud principal de la línea triple $L / N$. Líneas continuas: resultado del nuevo método basado en la medida de las fuerzas. Líneas discontinuas: resultado de la medida directa de los ángulos de contacto. 62

2.7. Porcentaje absoluto de las desviaciones relativas de las medidas del valor medio de los ángulos de contacto a partir del ángulo de contacto de Young para una gota de agua con $V=0,03 \mathrm{~cm}^{3}$ y $N=48$. Círculos: resultado del nuevo método basado en la medida de las fuerzas. Cuadrados: resultado de la medida directa de los ángulos de contacto.

2.8. Histéresis del ángulo de contacto para una gota de agua sobre una superficie de silicio recubierta de ácido poliláctico. a) Ángulo de contacto $\theta$ frente al tiempo. b) Radio de la línea triple $R$ frente al tiempo. c) Volumen $V$ frente al tiempo. Línea continua: resultados de la simulación con Surface Evolver. Línea discontinua: resultados teóricos obtenidos resolviendo la ecuación de Young-Laplace. Puntos: datos experimentales [43] . . . . . . . . . . . . . . . . . . . . . 
2.9. Ciclo de histéresis del ángulo de contacto para formamida sobre una superficie de silicio recubierta de ácido poliláctico-co-glicólico 50/50 . a) Ángulo de contacto $\theta$ frente al tiempo. b) Radio de la línea triple $R$ frente al tiempo. c) Volumen $V$ frente al tiempo. Línea continua: resultados de las simulaciones con Surface Evolver. Línea discontinua: resultados teóricos obtenidos a partir de la ecuación de YoungLaplace. Puntos: datos experimentales [43] . . . . . . . . . . . . .

2.10. Ciclo de histéresis del ángulo de contacto para formamida sobre una superficie de silicio recubierta de poli(metil metacrilato/n-butil metacrilato). a) Ángulo de contacto $\theta$ frente al tiempo. b) Radio de la línea triple $R$ frente al tiempo. c) Volumen $V$ frente al tiempo. Línea continua: resultados de las simulaciones con Surface Evolver. Línea discontinua: resultados teóricos obtenidos a partir de la ecuación de Young-Laplace. Puntos: datos experimentales [43].

2.11. Evolución temporal del ángulo de contacto de una gota de agua en un ciclo de histéresis. Línea continua: resultados obtenidos resolviendo la ecuación de Young-Laplace en coordenadas cilíndricas. Línea discontinua: resultados de simulación con Surface Evolver. Puntos: datos experimentales de Vargha-Butlet el al. [44]. a) DL-PLA 100; b) DL-PLGA 85/15, c) DL-PLGA 75/25, d) DL-PLGA 65/35; e) DL-PLGA 50/50.

3.1. Sección de la gota sésil sobre un substrato sólido con un determinado ángulo de inclinación $\alpha$. Los ángulos de contacto en la parte delantera (ángulos de avance $\theta_{\mathrm{av}}$ ) de la gota son diferentes que los de la parte posterior (ángulos de retroceso $\theta_{\mathrm{re}}$ ) . . . . . . . . . . . . . .

3.2. Aspecto de la base de la gota con diferentes formas de la línea de contacto triple: a) gota de base circular, b) gota de lados paralelos, y c) gota con asimetría de delante hacia atrás. 
3.3. Valores del factor de retención de la fuerza $k$ para la relación de aspecto $\beta=1$, obtenidos por los autores que lo han estudiado a lo largo de la historia, considerando diferentes suposiciones tanto en el modo de abordar el estudio como en la forma de la base de la gota. Los resultados de este trabajo (línea discontinua azul) se encuentran

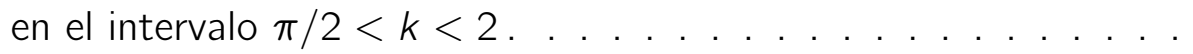

3.4. Planteamiento del problema para realizar el estudio de la inclinación del substrato en el que se encuentra depositada la gota sésil. a) Gota depositada en una superficie horizontal. b) Gota sobre un plano inclinado un ángulo $\alpha$ respecto a la horizontal. c) Gota sobre una superficie sin inclinación y con una componente horizontal de la gravedad gy

3.5. Forma de la gota durante el proceso de inclinación de la misma con el programa en Surface Evolver.

3.6. Componentes del peso al que se ve sometido una gota sobre un plano inclinado, según se ha seleccionado el sistema de ejes coordenados.

3.7. Resultados de Surface Evolver para una gota sobre un plano inclinado. a) Forma de la gota. b) Línea de contacto triple mostrando la posición de los vértices y las fuerzas de fricción que actúan sobre ellos (ver texto). c) Ángulos de contacto en función del ángulo $\phi$ definido en b). 84

3.8. Balance de fuerzas para una gota en un plano inclinado. Línea continua: contribución de la gravedad $\rho g V \operatorname{sen} \alpha$. Círculos: fuerza de retención total a lo largo de la dirección de inclinación $F_{y}$. Línea de puntos: Fuerza de retención total máxima estimada a partir de la ecuación (3.3) con $k=2$. Cuadrados: Fuerza de retención total máxima estimada durante la simulación. 
3.9. Fuerza de retención total adimensional $F_{y} / \sigma_{\mathrm{IV}} \omega$ frente a la diferencia de $\operatorname{cosenos} \Delta \cos =\cos \theta_{\min }-\cos \theta_{\max }$. a) Ángulo de contacto inicial igual a $\theta_{\mathrm{av}}$. b) Ángulo de contacto inicial igual a $\theta_{Y}$. Los símbolos son datos de la simulación. La línea continua son ajustes lineales de los datos, con pendientes a) $k=1,779$ y b) $k=1,573 \approx \pi / 2$. Línea discontinua: pendiente $k=2$. Línea de puntos: pendiente $k=\pi / 2$. La línea vertical punto-raya indica $\Delta \cos _{\max }=\cos \theta_{\mathrm{re}}-\cos \theta_{\mathrm{av}}$. . .

3.10. $\theta_{\min }$ (círculos) y $\theta_{\max }$ (cuadrados) frente al ángulo de inclinación $\alpha$. a) Ángulo de contacto inicial igual a $\theta_{\mathrm{av}}$. b) Ángulo de contacto inicial igual a $\theta_{Y}$. Los símbolos son datos de la simulación. La línea continua son ajustes lineales de los datos. La línea discontinua y la línea de puntos indican $\theta_{\mathrm{re}} \mathrm{y} \theta_{\mathrm{av}}$, respectivamente.

3.11. Pendiente $k$ frente a) al ángulo de contacto de retroceso $\theta_{\text {re }}, y$ b) al ángulo de contacto de avance $\theta_{\mathrm{av}}$

3.12. Pendiente $k$ frente a la diferencia entre ángulo de contacto de avance y retroceso $\Delta \theta=\theta_{\mathrm{av}}-\theta_{\mathrm{re}}$, para una gota de agua cuando se inclina progresivamente el substrato sobre el que se encuentra, con $\theta_{\mathrm{av}}=90^{\circ} .92$

3.13. Pendiente $k$ frente al volumen. Resultados de simulación con Surface Evolver para una gota de agua con $\Delta \theta=\theta_{\mathrm{av}}-\theta_{\mathrm{re}}=20^{\circ}$ y distintos ángulos de contacto de avance. . . . . . . . . . . . . . . . . . .

3.14. Fuerza $F_{\mathrm{y}} / \sigma_{\mathrm{lv}} \omega$ para una gota de agua, frente a la diferencia de cosenos de los ángulos de contacto $\Delta \cos =\cos \theta_{\min }-\cos \theta_{\max }$, con $\theta_{\mathrm{av}}-\theta_{\mathrm{re}}=20^{\circ}, \theta_{\mathrm{av}}=130^{\circ}$ y volúmenes distintos.

3.15. Resultados de Surface Evolver para gotas con diferentes ángulos de inclinación $\alpha$ con un ángulo de contacto inicial igual a $\theta_{\mathrm{av}}$. a) Línea de contacto triple. b) Ángulos de contacto en función de la coordenada $x$ perpendicular a la inclinación. Línea de puntos: $\alpha=0^{\circ}$, línea continua: $\alpha=10^{\circ}$, línea discontinua: $\alpha=20^{\circ}$, línea punto-raya:

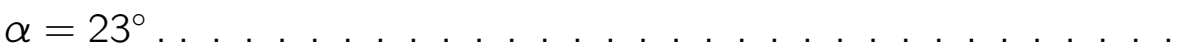


3.16. Resultados de Surface Evolver para gotas con diferentes ángulos de inclinación $\alpha$ y un ángulo de contacto inicial igual a $\theta_{Y}$. a) Línea de contacto triple. Línea de puntos: $\alpha \leq 18^{\circ}$, línea continua: $\alpha=21^{\circ}$, línea discontinua: $\alpha=23^{\circ}$, línea punto-raya: $\alpha=25^{\circ}$. b) Ángulos de contacto en función de la coordenada $x$ perpendicular a la inclinación. Línea de puntos: $\alpha=0^{\circ}$, línea continua: $\alpha=10^{\circ}$, línea punto-raya: $\alpha=18^{\circ}$, línea discontinua: $\alpha=21^{\circ}$, línea punto-raya: $\alpha=25^{\circ} \ldots$.

3.17. Comparación entre los resultados de la simulación y los datos experimentales para $\mathrm{Sn}-\mathrm{Ag}-\mathrm{Cu}$ fundido sobre una substrato inclinado de cobre. a) $\theta_{\min }$ (círculos) y $\theta_{\max }$ (cuadrados) frente al ángulo de inclinación $\alpha$. Las líneas continuas son guías para la observación. Las líneas discontinuas y las líneas de puntos indican $\theta_{\mathrm{re}} \mathrm{y} \theta_{\mathrm{av}}$ respectivamente. b) $\cos \theta_{\min }$ (círculos) y $\cos \theta_{\max }$ (cuadrados) frente al ángulo de inclinación $\alpha$. Las líneas continuas son ajustes lineales de los datos. Las líneas discontinuas y las líneas de puntos indican $\cos \theta_{\text {re }}$ y $\cos \theta_{\text {av }}$ respectivamente. En ambas figuras los símbolos rellenos son los datos experimentales [67]. . . . . . . . . . . . . . . .

3.18. Relación de aspecto $\beta$ frente $\Delta \cos$, para $\theta_{\mathrm{re}}-\theta_{\mathrm{av}}=25^{\circ}$, con $\theta_{\mathrm{av}}=$ $120^{\circ}$. Puntos: resultado de simulación con Surface Evolver. Línea: ajuste por mínimos cuadrados de todos los datos. . . . . . . . . . . 104

3.19. Relación de aspecto $\beta$ frente a $\Delta \cos$, para $\theta_{\text {re }}-\theta_{\mathrm{av}}=25^{\circ}$, con $\theta_{\mathrm{av}}=60^{\circ}$. Puntos: resultado de simulación con Surface Evolver. Línea: ajuste por mínimos cuadrados de los datos iniciales.

3.20. Pendiente $\hat{k}$ frente a) al ángulo de contacto de retroceso $\theta_{\text {re }}, y$ b) al

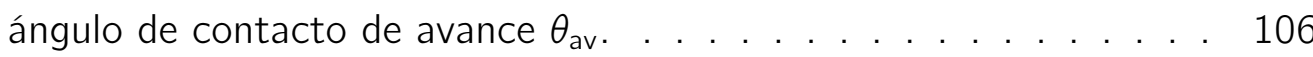

3.21. Pendiente $\hat{k}$ frente al volumen para una gota de agua. Resultados de simulación con Surface Evolver manteniendo $\Delta \theta=\theta_{\mathrm{av}}-\theta_{\mathrm{re}}=20^{\circ} \mathrm{y}$ considerando distintos ángulos de contacto de avance. 
3.22. Línea de contacto triple de una gota cuando el plano sobre el que se deposita está inclinado un ángulo $0^{\circ}<\alpha<_{c}$, tiene forma aproximadamente semicircular (sombreado azul) en la parte superior y aproximadamente semielíptica (sombreado verde) en la inferior.

3.23. Semiancho $\omega$ (puntos azules), radio de la base de la gota obtenido a partir del área de contacto $R_{\mathrm{a}}$ (puntos rojos) y producto del semiancho por la raíz cuadrada de la relación de aspecto $\omega \cdot \sqrt{\beta}$ (puntos verdes), frente al al ángulo de inclinación $\alpha$ para una gota de agua de $V=90 \mu \mathrm{l}, \theta_{\min }-\theta_{\max }=20^{\circ}$ y $\theta_{\mathrm{av}}=70^{\circ}$.

3.24. Fuerza $F_{y} / \sigma \omega$ frente al cociente de áreas $A / A_{0}$ (puntos rojos), para una inclinación desde $\alpha=0^{\circ}$ a $\alpha=36^{\circ}$. Resultado de la simulación con Surface Evolver para una gota de agua de $V=18 \mu \mathrm{l}, \theta_{\mathrm{av}}=90^{\circ}$ y $\Delta \theta=\theta_{\text {re }}-\theta_{\mathrm{av}}=20^{\circ}$. La recta azul es un ajuste lineal de los datos. 110

3.25. Fuerza $F_{\mathrm{y}} / \sigma \omega$ frente al cociente de áreas $A / A_{0}$, para una gota de agua inclinada desde $\alpha=0^{\circ}$. Resultado de la simulación con Surface Evolver para $\theta_{\mathrm{av}}=130^{\circ}, \Delta \theta=\theta_{\mathrm{av}}-\theta_{\mathrm{re}}=20^{\circ}$ y diferentes volúmenes. Las rectas representan ajustes lineales de los datos.

3.26. Fuerza $F_{\mathrm{y}} / \sigma \omega$ frente al cociente de áreas $A / A_{0}$, para una gota de agua inclinada desde $\alpha=0^{\circ}$. Las líneas de puntos son el resultado de la simulación con Surface Evolver para diferentes $\theta_{\mathrm{av}}$ y $\Delta \theta=$ $\theta_{\mathrm{av}}-\theta_{\mathrm{re}}=5^{\circ}$ y $V=36 \mu \mathrm{l}$. Las rectas representan ajustes lineales de los datos para cada $\theta_{\mathrm{av}}$.

3.27. Pendiente $k / \hat{k}$ en función del ángulo de contacto de avance $\theta_{\mathrm{av}}$, para diversos $\Delta \theta=\theta_{\mathrm{re}}-\theta_{\mathrm{av}} \operatorname{con} V=36 \mu \mathrm{l}$.

3.28. Cociente $k / \hat{k}$ respecto al volumen para una gota de agua. Resultados de simulación con Surface Evolver para $\Delta \theta=20^{\circ}$ y diferentes valores de $\theta_{\mathrm{av}} \ldots \ldots \ldots \ldots \ldots \ldots \ldots \ldots \ldots$

4.1. Ángulos de contacto mínimo (círculos) y máximo (cuadrados) frente a la inclinación del substrato, para diferentes valores del ángulo de contacto inicial, en una simulación de placa inclinable para una gota de agua de $V=150 \mu \mathrm{l}$ sobre PTFEr con $\theta_{\mathrm{re}}=87,9^{\circ}$ y $\theta_{\mathrm{av}}=126^{\circ}$. 
4.2. Ángulos de inclinación críticos $\alpha_{1}$ y $\alpha_{2}$ frente al ángulo de contacto inicial. Simulación de placa inclinable para una gota de agua de $V=$ $150 \mu \mathrm{l}$ sobre PTFEr. Las rectas representan el ajuste por mínimos cuadrados de los datos.

4.3. Ángulos de inclinación críticos $\alpha_{1}$ y $\alpha_{2}$ frente al ángulo de contacto inicial. Simulación de placa inclinable para una gota de agua de $V=150 \mu \mathrm{l}$ sobre PP. Las rectas representan el ajuste por mínimos cuadrados de los datos. . . . . . . . . . . . . . . . . . .

4.4. Diferencia entre el ángulo de inclinación crítico de la parte posterior $\alpha_{\mathrm{u}}$ y frontal $\alpha_{\mathrm{d}}$, frente al ángulo de contacto inicial. Simulación de placa inclinable para una gota de agua de $V=150 \mu \mathrm{l}$ sobre PTFEr. La recta es un ajuste lineal de los datos.

4.5. Diferencia entre los ángulos de inclinación críticos máximo y mínino, frente al ángulo de contacto inicial. Simulación de placa inclinable para una gota de agua de $V=150 \mu$ l sobre PTFEr. Las rectas son ajustes lineales de los datos.

4.6. a) Ángulo de inclinación crítico mínino $\alpha_{1}$ y b) diferencia de ángulos de inclinación críticos de la parte posterior y frontal de la zona de contacto $\alpha_{\mathrm{u}}-\alpha_{\mathrm{d}}$, frente al ángulo de contacto inicial $\theta_{0}$ para simulaciones de una gota de agua de volumen $100 \mu$ l (cuadrados verdes), $120 \mu \mathrm{l}$ (círculos rojos) y $150 \mu \mathrm{l}$ (triángulos azules) sobre uPVC. Las rectas representan ajustes lineales de los datos. El máximo de los valores en a) y la intersección de cada línea con el eje de abscisas en b) debe corresponder al ángulo de contacto más estable.

4.7. Resultados del método de simulación de vibraciones para una gota de agua de $V=150 \mu \mathrm{l}$ sobre PTFEr. Ángulo de contacto inicial $\theta_{0}=111,71^{\circ}$. a) Función oscilante. b) Ángulos de contacto. c) Radio de la gota. 
4.8. Resultados del método de simulación de vibraciones para una gota de agua de $V=150 \mu$ l sobre PTFEr. a) Ángulos de contacto y b) Radio de contacto. Ángulo inicial $\theta_{0}: 87,9^{\circ}$ rojo, 92,66 naranja, 97,42 ${ }^{\circ}$ amarillo, $102,18^{\circ}$ marrón, $106,95^{\circ}$ verde, $111,71^{\circ}$ rosa, $116,47^{\circ}$ azul claro, $121,23^{\circ}$ azul oscuro y $126^{\circ}$ gris.

4.9. Valor absoluto de la diferencia entre los ángulos de contacto después de la vibración y los ángulos de contacto iniciales, en función del ángulo de contacto inicial. Simulación de vibración para una gota de agua de $V=150 \mu \mathrm{l}$ sobre PTFEr. Las rectas son ajustes lineales de los datos.

4.10. Valor absoluto de la variación de los ángulos de contacto después de la vibración en términos del ángulo de contacto inicial para una gota de agua sobre uPVC. Círculos verdes: $V=50 \mu \mathrm{l}$, triángulos rojos: $V=150 \mu l$.

4.11. a) Diferencia de los ángulos de inclinación críticos frente al ángulo de contacto inicial, simulación de placa inclinable (círculos). b) Valor absoluto de la variación de los ángulos de contacto después de la vibración en términos del ángulo de contacto inicial, simulación de vibración (cuadrados). Ambas para una gota de agua de $V=150 \mu \mathrm{l}$ sobre PTFEs.

A.1. Variables que se utilizan para expresar la ecuación de Young-Laplace en coordenadas cilíndricas. 



\section{Índice de cuadros}

1.1. Valores típicos de la tensión superficial de algunos líquidos puros a temperatura de $20^{\circ} \mathrm{C}[3,8] \ldots \ldots . \ldots . \ldots 23$

3.1. Valores del factor de retención de la fuerza $k$ a lo largo de la historia. 79

4.1. Ángulos de contacto de avance y retroceso [46], ángulo de contacto más estable obtenido a partir de la media aritmética de estos valores (4.1) así como el valor obtenido de la media de los cosenos (4.2), y los resultados obtenidos mediante simulación para el método de placa inclinable utilizando los procedimientos A y B. Se considera una gota de agua de $150 \mu$ l sobre varios polímeros.

4.2. Valores del ángulo de contacto más estable para gotas de agua de diferentes volúmenes sobre uPVC; obtenidos por el método $A\left(\theta_{\text {tilt }}^{A}\right)$ y por el método B $\left(\theta_{\text {tilt }}^{\mathrm{B}}\right)$. . . . . . . . . . . . . . . . . . . 130

4.3. Ángulos de contacto de avance y retroceso [46], ángulo de contacto más estable obtenido a partir de la media aritmética de estos valores (4.1) así como el valor obtenido de la media de los cosenos (4.2), y los resultados obtenidos mediante simulación para el método de vibración utilizando los procedimientos A y B. Se considera una gota de agua de $150 \mu$ l sobre varios polímeros.

4.4. Valor del ángulo de contacto más estable determinado mediante el método de vibración, para dos volúmenes diferentes de la gota de agua sobre $u P V C$; obtenidos por el método $A\left(\theta_{\text {vib }}^{A}\right)$ y por el método B $\left(\theta_{\text {vib }}^{B}\right)$ 
4.5. Ángulos de contacto de avance y retroceso, media aritmética de estos valores (4.1) así como el valor obtenido de la media de los cosenos (4.2), el ángulo de contacto más estable medido experimentalmente [46] y mediante simulaciones por medio de los métodos de placa inclinable y de vibración. Se considera una gota de agua de $150 \mu \mathrm{l}$ sobre varios polímeros. . . . . . . . . . . . . . . . . . . 142 